\title{
A New Framework For Helicopter Vibration Suppression; Time-Periodic System Identification and Controller Design
}

\author{
Thesis by
}

Fatma Demet Ulker, M.Sc.

A thesis submitted to the Faculty of Graduate Studies and Research in partial fulfillment of the requirements

for the degree of

\section{Doctor of Philosophy}

Ottawa-Carleton Institute for Mechanical and Aerospace Engineering

\author{
Department of Mechanical and Aerospace Engineering \\ Carleton University \\ Ottawa, Ontario, Canada
}

April,2011

(C) Copyright by Fatma Demet Ulker, 2011 


$\begin{array}{ll}\begin{array}{l}\text { Library and Archives } \\ \text { Canada }\end{array} & \begin{array}{l}\text { Bibliothèque et } \\ \text { Archives Canada }\end{array} \\ \begin{array}{l}\text { Published Heritage } \\ \text { Branch }\end{array} & \begin{array}{l}\text { Direction du } \\ \text { Patrimoine de l'édition }\end{array} \\ \begin{array}{l}\text { 395 Wellington Street } \\ \text { Ottawa ON K1A ON4 } \\ \text { Canada }\end{array} & \begin{array}{l}395, \text { rue Wellington } \\ \text { Ottawa ON K1A ON4 } \\ \text { Canada }\end{array}\end{array}$

Your file Votre référence ISBN: 978-0-494-81546-5

Our file Notre référence

ISBN: 978-0-494-81546-5

NOTICE:

The author has granted a nonexclusive license allowing Library and Archives Canada to reproduce, publish, archive, preserve, conserve, communicate to the public by telecommunication or on the Internet, loan, distribute and sell theses worldwide, for commercial or noncommercial purposes, in microform, paper, electronic and/or any other formats.

The author retains copyright ownership and moral rights in this thesis. Neither the thesis nor substantial extracts from it may be printed or otherwise reproduced without the author's permission.
AVIS:

L'auteur a accordé une licence non exclusive permettant à la Bibliothèque et Archives Canada de reproduire, publier, archiver, sauvegarder, conserver, transmettre au public par télécommunication ou par l'Internet, prêter, distribuer et vendre des thèses partout dans le monde, à des fins commerciales ou autres, sur support microforme, papier, électronique et/ou autres formats.

L'auteur conserve la propriété du droit d'auteur et des droits moraux qui protège cette thèse. $\mathrm{Ni}$ la thèse ni des extraits substantiels de celle-ci ne doivent être imprimés ou autrement reproduits sans son autorisation.
In compliance with the Canadian Privacy Act some supporting forms may have been removed from this thesis.

While these forms may be included in the document page count, their removal does not represent any loss of content from the thesis.
Conformément à la loi canadienne sur la protection de la vie privée, quelques formulaires secondaires ont été enlevés de cette thèse.

Bien que ces formulaires aient inclus dans la pagination, il n'y aura aucun contenu manquant. 
The undersigned hereby recommend

to the Faculty of Graduate and Postdoctoral Affairs

acceptance of the thesis

\title{
A New Framework For Helicopter Vibration Suppression; Time-Periodic System Identification and Controller Design
}

\author{
submitted by Fatma Demet Ulker
}

in partial fulfillment of the requirements for the degree of

\section{Doctor of Philosophy}

Professor Fred Nitzsche, Thesis Supervisor

Professor Sypridon Voutsinas, Thesis Co-supervisor

Professor Norman Wereley, External Examiner

Professor Metin I. Yaras, Chair

Carleton University

2011 


\section{Abstract}

In forward flight, helicopter rotor blades function within a highly complex aerodynamic environment that includes both near-blade and far-blade aerodynamic phenomena. These aerodynamic phenomena cause fluctuating aerodynamic loads on the rotor blades. These loads when coupled with the dynamic characteristics and elastic motion of the blade create excessive amount of vibration. These vibrations degrade helicopter performance, passenger comfort and contributes to high cost maintenance problems. In an effort to suppress helicopter vibration, recent studies have developed active control strategies using active pitch links, flaps, twist actuation and higher harmonic control of the swash plate. In active helicopter vibration control, designing a controller in a computationally efficient way requires accurate reduced-order models of complex helicopter aeroelasticity. In previous studies, controllers were designed using aeroelastic models that were obtained by coupling independently reduced aerodynamic and structural dynamic models. Unfortunately, these controllers could not satisfy stability and performance criteria when implemented in highfidelity computer simulations or real-time experiments. In this thesis, we present a novel approach that provides accurate time-periodic reduced-order models and time-periodic $H_{2}$ and $H_{\infty}$ controllers that satisfy the stability and performance criteria. Computational efficiency and the necessity of using the approach were validated by implementing an actively controlled flap strategy.

In this proposed approach, the reduced-order models were directly identified from highfidelity coupled aeroelastic analysis by using the time-periodic subspace identification method. Time-periodic $\mathrm{H}_{2}$ and $H_{\infty}$ controllers that update the control actuation at every time step were designed. The control synthesis problem was solved using Linear Matrix Inequality and periodic Riccati Equation based formulations, for which an in-house periodic Riccati solver was developed.

The results show that first, important helicopter aeroelastic features can only be captured using high-fidelity coupled aeroelastic analysis; ignoring these features through uncoupled analysis leads to closed-loop performance degradation and instabilities. Second, timeperiodic models are necessary to obtain an accurate map between control actuation and helicopter aeroelastic response; time-invariant models fail to provide accurate prediction. 
Third, time-Periodic $H_{2}$ and $H_{\infty}$ controllers satisfy the stability and design performance criteria when implemented in high-fidelity aeroelastic analysis. Finally, we propose robust $H_{2}$ and $H_{\infty}$ controller design strategies that are capable of modeling variable advance ratios. 


\section{Acknowledgements}

First and foremost, I would like to thank my supervisor, Professor Fred Nitzsche for his academic guidance and financial support throughout the entire research period. For many aspects, he contributed to my wonderful experience as being an international student in Canada.

I greatly appreciate Professor Spyridon Voutsinas and Dr. Vasilis Riziots at the National Technical University of Athens for the time that they give me to develop the aeroelastic code.

I am grateful to Michael Rose, German Aerospace Center (DLR) for his interest in my research and collaboration on the development of the discrete-time periodic Riccati solver.

I would like to acknowledge the financial support given by the Ontario Center of Excellence during my four-month visit to National Technical University of Athens, Greece.

On a personal note, for sharing many valuable thoughts and incredibly fun moments, I would like to thank Maciej Balajewicz. My deep gratitude goes to my sister, Dr. Buket Ülker, who has been a role model and source of inspiration. Finally, i would like to thank my parents for all their encouragement, support, and strength. 


\section{Table of Contents}

$\begin{array}{ll}\text { Abstract } & \text { iii }\end{array}$

$\begin{array}{lll}\text { Acknowledgements } & \text { v }\end{array}$

Table of Contents $\quad$ vi

List of Figures $\quad x$

List of Tables $\quad$ xvi

List of Abbreviations $\quad$ xviii

List of Symbols $\quad$ xx

1 Sources of Helicopter Vibration and Means of Control $\quad 1$

1.1 Overview of the Comprehensive and Approximate Modeling of Helicopter Aerodynamics and Structural Dynamics . . . . . . . . . . 6

1.2 Modeling and System Identification of Time-Periodic Systems . . . . . . . . 9

1.2.1 Brief Introduction of LTP Systems and Input/Output Mapping . . . 9

1.2.2 Historical Development of Identification of Time Periodic Systems . 14

1.2.3 LTP Modeling of Helicopter Rotor Response . . . . . . . . . . . . . 18

1.3 Controller Synthesis for LTP Systems . . . . . . . . . . . . . . . . . 20

1.3.1 Literature Survey on Controller Design For Helicopter Vibration Sup-

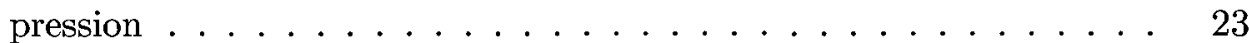

1.4 Proposed Active Vibration Control Validation Case . . . . . . . . . . . . . 25

1.5 Thesis Objectives . . . . . . . . . . . . . . . . 27 
1.6 Main Contributions . . . . . . . . . . . . . . . . 27

1.7 Organization of the Thesis . . . . . . . . . . . . 28

2 Aeroelastic Code and Simulations $\quad 30$

2.1 Introduction . . . . . . . . . . . . . . . . 30

2.2 Aeroelastic Code . . . . . . . . . . . . . . . . . . . . 30

2.3 Simulations for Baseline Case . . . . . . . . . . . . . . 37

2.3 .1 SHARCS Blade . . . . . . . . . . . . . . . 37

2.3 .2 Baseline Flight Condition . . . . . . . . . . . . 41

2.4 Assumptions for Baseline Flight Case and Trailing Edge Flap Modeling . 46

2.5 Conclusion . . . . . . . . . . . . . . . . . 46

3 Background of the Applied Theory: System Identification and Controller $\begin{array}{ll}\text { Design for Time Periodic Systems } & 48\end{array}$

3.1 Introduction . . . . . . . . . . . . . . . . . . 48

3.2 Modeling . . . . . . . . . . . . . . . . . 48

3.2.1 System Definition . . . . . . . . . . . . . . . 49

3.3 Overview on Linear Time Periodic Systems . . . . . . . . . . . . 50

3.4 State-Space Realization from Ensemble Input/Output Data Pairs . . . . . . 52

3.5 Stability of LTP Systems . . . . . . . . . . . . . . 56

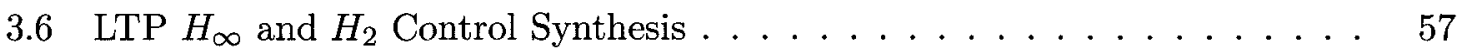

3.6.1 Discrete-Time Time-Periodic $H_{\infty}$ Controller Synthesis $[88,104,105,125] 60$

3.6.2 Discrete-Time Time-Periodic $H_{2}$ Controller Synthesis [48,50,89,110] 63

3.7 Solution of Discrete-Time Periodic Riccati Equation . . . . . . . . . . 65

4 Identification of Helicopter Rotor Blade Aeroelastic Response $\quad 69$

4.1 Introduction . . . . . . . . . . . . . . . . . . 69

4.2 System Identification as a Design Problem: Input/output Selection . . . . . 69

4.2.1 Sensitivity Analysis: Effect of TEF Deflection on the Hub Loads and Blade Loads . . . . . . . . . . . . . . . . . . . . 71 
4.3 Linearity, Periodicity, and Equivalency of the Helicopter Rotor Blade's Aeroelastic Response . . . . . . . . . . . . . . . 76

4.4 System Identification as a Design Problem: Model Set \& Simulation Design 84

4.5 Discrete-Time Time-Periodic State-Space Realization using Subspace Identification Part I: One Blade Analysis . . . . . . . . . . . . . . . . . 86

4.5.1 Stability of the System . . . . . . . . . . . . . 88

4.5 .2 Validation Simulations . . . . . . . . . . . . . . . 89

4.6 Discrete-Time Time-Periodic State-Space Realization using Subspace Identification Part II: All Blade Analysis . . . . . . . . . . . . . . . 95

4.6 .1 Stability of the System . . . . . . . . . . . . 96

4.6 .2 Validation Simulations . . . . . . . . . . . . . . . . 98

4.7 Aerodynamic and Aeroelastic Analysis of Trailing Edge Flap Deflection . . 108

4.8 Conclusion . . . . . . . . . . . . . . . . . 117

5 LTP Controller Synthesis and Implementation For Helicopter Vibration $\begin{array}{ll}\text { Suppression } & 119\end{array}$

5.1 Introduction . . . . . . . . . . . . . . . . . . . . 119

5.2 Formulation of the Controller Synthesis Problem for Helicopter Vibration

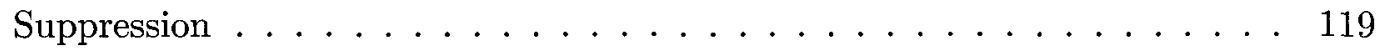

5.2.1 Controller Synthesis Problem Formulation: One Blade Analysis . . . 121

5.2.2 Controller Synthesis Problem Formulation: All Blades Analysis . . . 123

5.3 Disturbance Modeling and Performance Selection . . . . . . . . . . . 123

5.4 LTP $H_{\infty}$ Controller Synthesis and Closed-Loop Control Simulations . . . . 125

5.4.1 One Blade Analysis: Suppression of the Rotating Frame Blade Load,

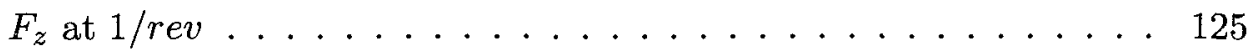

5.5 Implementation Problems and Propositions . . . . . . . . . . . . 130

5.6 LTP $\mathrm{H}_{2}$ Controller Synthesis and Closed-Loop Control Simulations . . . . . 132

5.6.1 One Blade Analysis: Suppression of the Rotating Frame Blade Load,

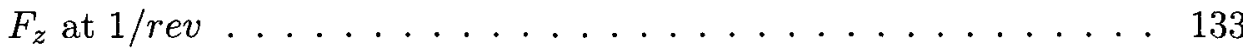

5.6.2 All Blade Analysis: Suppression of the Rotating Frame Blade Load, $F_{z}$ at $4 / r e v \ldots \ldots \ldots \ldots \ldots$ 
5.6.3 All Blade Analysis: Suppression of the Rotating Frame Blade Pitch Moment, $M_{y}$ at $3 / r e v \ldots \ldots \ldots \ldots \ldots$

5.7 Closed-Loop Control Results for Different Forward Flight Cases . . . . . . . 149

5.7.1 Closed-Loop Control Results for Forward Flight Case with Advance Ratio, $\mu=0.224 \ldots \ldots \ldots \ldots \ldots$

5.7.2 Closed-Loop Control Results for Forward Flight Case with Advance Ratio, $\mu=0.17 \ldots \ldots \ldots \ldots \ldots$. . . . . . . . . . . . . . . .

5.8 Conclusion . . . . . . . . . . . . . . . . . 159

6 Conclusions and Recommendations 162

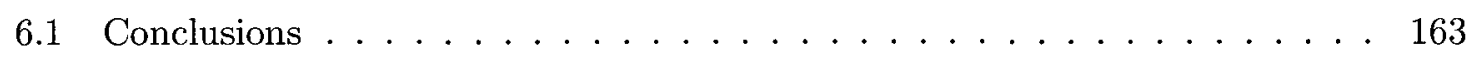

6.1.1 Important Aeroelastic Features of Helicopters Can Only be Captured With High-Fidelity Coupled Aeroelastic Analysis . . . . . . . . . 163

6.1.2 Time-Periodic Models are Necessary to Obtain an Accurate Map Between Control Actuation and Helicopter Aeroelastic Response . . . . 164

6.1.3 Time-Periodic $H_{2}$ and $H_{\infty}$ Controllers Satisfy the Stability and Design Performance Criteria When Implemented in High-Fidelity Aeroelastic Analysis . . . . . . . . . . . . . . . . 165

6.2 Recommendations . . . . . . . . . . . . . . 166

6.2 .1 Numerical-Analytical Improvements . . . . . . . . . . 166

6.2 .2 Modifications for Real-time Experiments . . . . . . . . . . 167

A Background of LMIs and Block Diagonal Representation $\quad 169$

A.1 Linear Matrix Inequality . . . . . . . . . . . . . . . . . . . . 169

A.2 Block-Diagonal Operator . . . . . . . . . . . . 170

$\begin{array}{ll}\text { References } & 171\end{array}$ 


\section{List of Figures}

1.1 Schematic representation of aerodynamic environment around helicopter in

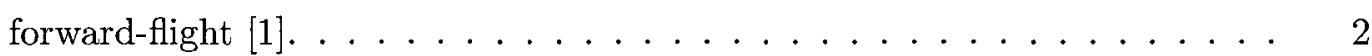

1.2 Traditional approach for active vibration control of helicopters. . . . . . . . 3

1.3 Proposed approach for active vibration control of helicopters. . . . . . . . . 5

1.4 Comparison of input-output relations for LTI and LTP systems. . . . . . . . 10

1.5 Time-lifting of continuous time signals $[50,62] \ldots \ldots \ldots \ldots$

1.6 General feedback diagram for controller synthesis problem [89]. . . . . . . . 20

1.7 Controller synthesis; direct versus indirect way $[103] \ldots \ldots 22$

1.8 The SHARCS blade with three individual control means [16] . . . . . . . 26

2.1 Representation of airfoil and its wake. . . . . . . . . . . 31

2.2 Representation of the blade and its wake with vortex sheets and formation of the vortex particle. ............................ 33

2.3 Illustrative example of wake with sinusoidal TEF deflection. . . . . . . . 34

2.4 A schematic diagram of the coordinate frames used by the structural com-

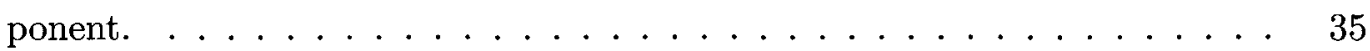

2.5 Definition of the finite element. . . . . . . . . . . 35

2.6 Definition of rotor and blade axis of reference in the GENUVP/GAST aeroelastic code. . . . . . . . . . . . . . . . 37

2.7 The SHARCS blade with three individual feedback systems [16](repeated). 38

2.8 Geometric properties of SHARCS blade. . . . . . . . . . . . . 38

2.9 Normalized mode shapes at the eigenfrequencies. . . . . . . . . . . . . 40

2.9 Normalized mode shapes at the eigenfrequencies (Cnt) . . . . . . . . . 41

2.10 Convergence of the baseline case: hub loads, $F_{X}, F_{Y}, F_{Z}, M_{X}, M_{Y}, M_{Z} \ldots$. 43 
2.11 Rotating blade loads and hub loads at baseline case. . . . . . . . . . .

3.1 State-space modeling of time-varying systems [121] . . . . . . . . . . . 51

3.2 General feedback block diagram representation. . . . . . . . . . . 58

3.3 Lower linear fractional transformation form . . . . . . . . . . . . . . 58

4.1 Definition of rotor and blade axis of reference in GENUVP/GAST (repeated). 70

4.2 Physically realizable TEF deflection,$\delta_{1} \ldots \ldots \ldots \ldots \ldots \ldots$

4.3 Comparison of the helicopter rotor system's aeroelastic response in terms of hub loads, $F_{X}, F_{Y}, F_{Z}$ and moments, $M_{X}, M_{Y}, M_{Z}$ to the physically realizable TEF deflection $\delta_{1} \ldots \ldots \ldots \ldots \ldots \ldots \ldots \ldots \ldots \ldots \ldots$

4.4 Comparison of the helicopter rotor system's aeroelastic response in terms blade loads $F_{x, 1}, F_{y, 1}, F_{z, 1}$ and moments $M_{x, 1}, M_{y, 1}, M_{z, 1}$ to the physically realizable TEF deflection $\delta_{1} \ldots \ldots \ldots \ldots \ldots \ldots$

4.5 Identification setup for the helicopter rotor system's aeroelastic response under TEF deflection. . . . . . . . . . . . . . . . . . .

4.6 Linearity of the net vibratory blade loads $F_{z, 1,2,3,4}$ caused by the TEF de-

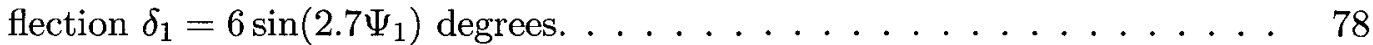

4.7 Linearity of the net vibratory blade loads $M_{y, 1,2,3,4}$ caused by the TEF de-

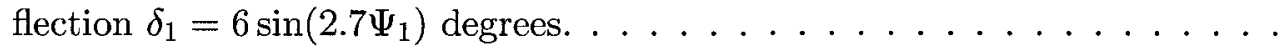

4.8 Comparison of helicopter rotor system's aeroelastic impulse responses in terms of blade load, $F_{z, 1}, M_{y, 1}$ to a TEF deflection of $4^{o}$ amplitude at azimuth locations $\Psi=90^{\circ}, \Psi=270^{\circ}$ and same azimuth location after a full revolution. 81

4.9 Comparison of aeroelastic impulse responses of the helicopter rotor system in terms of blade loads $F_{z, 1,3}, M_{y, 1,3}$ to a TEF deflection of $4^{\circ}$ amplitude at azimuth locations $\Psi=90^{\circ}, \Psi=270^{\circ} \ldots \ldots \ldots \ldots$

4.10 The GENUVP/GAST simulation setup for system identification. . . . . . . 86

4.11 Singular Values of the output Hankel matrix $R_{22} \ldots \ldots \ldots$. . . . . . . 87

4.12 Relative percentage $L_{2}$ error norm between the blade loads $F_{z, 1}, M_{y, 1}$ obtained from the GENUVP/GAST simulation and system model of different

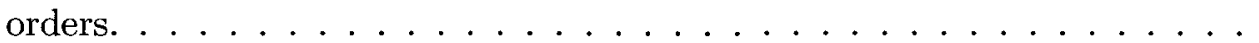


4.13 Distribution of the eigenvalues at azimuth Angles $\Psi=0,90,180,270$ degrees and the characteristic multipliers of the system. . . . . . . . .

4.14 Comparison of LTP system assumption and LTI system assumption: blade loads, $F_{z, 1}$ and $M_{y, 1}$ to a physically realizable random TEF deflection obtained from LTP and LTI reduced-order models, and the GENUVP/GAST simulations. . . . . . . . . . . . . . . . . .

4.15 Comparison of blade loads $F_{z, 1}$ and $M_{y, 1}$ obtained from LTP system model and the GENUVP/GAST aeroelastic simulations when the TEF of one blade was deflected at a frequency of $1 /$ rev.3/rev and $5 /$ rev, respectively. . . .

4.15 Comparison of blade loads $F_{z, 1}$ and $M_{y, 1}$ obtained from LTP system model and the GENUVP/GAST aeroelastic simulations when the TEF of one blade was deflected at a frequency of $1 /$ rev, $3 / r e v$ and $5 /$ rev respectively. (Cnt.)

4.16 Distribution of the eigenvalues at azimuth angles, $\Psi=0,90,180,270$ degrees and the characteristic multipliers of the system. . . . . . . . .

4.17 Comparison of the blade loads obtained from the LTP models of the one blade analysis (1BA) with order $n_{k}=24$, and the all blades analysis (ABA) with order $n_{k}=48$, and the GENUVP/GAST aeroelastic simulations when the TEF of all blades was deflected with a phase shift $\phi_{2}=90^{\circ}$ at $1 /$ rev

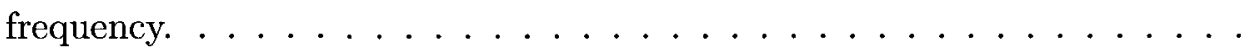

4.18 Comparison of the hub loads obtained from the LTP models of the one blade analysis (1BA) with order $n_{k}=24$, and the all blade analysis (ABA) with order $n_{k}=48$, and the GENUVP/GAST aeroelastic simulations when the TEF of all blades was deflected with a phase shift $\phi_{\imath}=90^{\circ}$ at $1 /$ rev frequency. 100

4.19 Phase-shifted TEF deflections at $3 /$ rev frequency $\left(\delta_{\imath}=6 \sin \left(3 \Omega+\phi_{\imath}\right)\right.$ degrees. $) 103$

4.20 Comparison of the blade loads obtained from the LTP models of the one blade analysis $(1 \mathrm{BA})$ with order $n_{k}=24$, and the all blade analysis (ABA) with order $n_{k}=48$, and the GENUVP/GAST aeroelastic simulations when the TEF of all blades was deflected with a phase shift $\phi_{\imath}$ at $3 /$ rev frequency. 104 
4.21 Comparison of the hub loads obtained from the LTP models of the one blade analysis (1BA) with order $n_{k}=24$, and the all blade analysis (ABA) with order $n_{k}=48$, and the GENUVP/GAST aeroelastic simulations when the TEF of all blades was deflected with a phase shift $\phi_{2}$ at $3 / r$ rev frequency. .

4.22 Relative percentage error norm $L_{2}$ between the blade loads $F_{z, 1}$ obtained from GENUVP/GAST simulation and identified model of different orders for the one blade analysis and the all blades analysis. . . . . . . . . . . . 107

4.23 Sectional lift coefficient. . . . . . . . . . . . . . . . . . . . . 109

4.24 Sectional moment coefficient. . . . . . . . . . . . . . . . . 110

4.25 Wake of the four-bladed helicopter rotor system in forward flight. . . . . . . 112

4.26 Comparison of the blade loads obtained from the GENUVP/GAST aeroelastic simulations for SHARCS blade and the softened blade when the TEF of all blades was deflected with $\phi_{2}=90^{\circ}$ phase shift at $1 /$ rev frequency. . .

4.27 Comparison of the blade loads obtained from the GENUVP/GAST aeroelastic simulations for SHARCS blade and the softened blade when the TEF of all blades was deflected with $\phi_{\imath}=90^{\circ}$ phase shift at $3 /$ rev frequency. . .

5.1 General feedback block diagram representation. . . . . . . . . . . . 120

5.2 Linear fractional transformation form (repeated) [89]. . . . . . . . . . . 121

5.3 Control of $1 /$ rev rotating frame blade load, $F_{z} . H_{\infty}$ controller design performance and disturbance weights. . . . . . . . . . . .

5.4 Control of $1 /$ rev rotating frame blade load, $F_{z}$. Control command given to

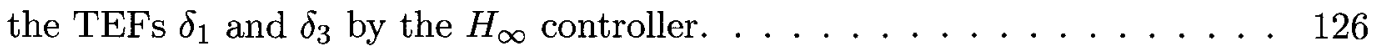

5.5 Control of $1 /$ rev rotating frame blade load, $F_{z}$. Closed-loop control simulations: comparison between the baseline and controlled blade loads, $F_{z}$ obtained from the reduced-order model and the GENUVP/GAST simulations.128

5.6 Control of $1 /$ rev rotating frame blade load, $F_{z}$. Closed-loop control simulations: comparison between the baseline and controlled hub loads, $F_{Z}, M_{X}$, and $M_{Y}$ obtained from the reduced-order model and the GENUVP/GAST

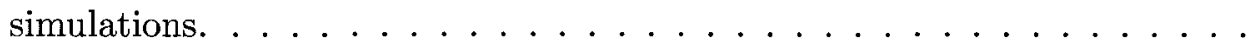


5.7 Control of $1 /$ rev rotating frame blade load, $F_{z} . H_{2}$ controller design performance and disturbance weights. . . . . . . . . . . . . . . 134

5.8 Control of $1 /$ rev rotating frame blade load, $F_{z}$. Control commands given to

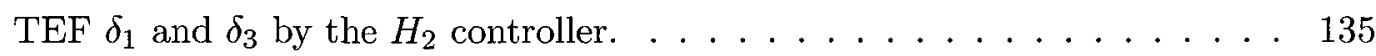

5.9 Control of $1 /$ rev rotating frame blade load, $F_{z}$. Closed-loop control simulations: comparison between the baseline and controlled blade loads, $F_{z}$ obtained from the reduced-order model and the GENUVP/GAST simulations.136

5.10 Control of $1 /$ rev rotating frame blade load, $F_{z}$. Closed-loop control simulations: comparison between the baseline and controlled hub loads, $F_{Z}, M_{X}$ and $M_{Y}$ obtained from the reduced-order model and the GENUVP/GAST

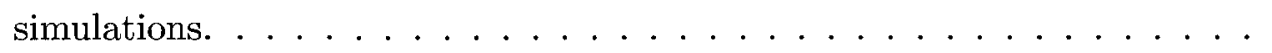

5.11 Control of $4 /$ rev rotating frame blade load, $F_{z} . H_{2}$ controller design performance and disturbance weights. . . . . . . . . . . . . .

5.12 Control of $4 /$ rev rotating frame blade load, $F_{z}$. Control command given to all TEFs $\delta_{1,2,3,4}$ by the $H_{2}$ controller. . . . . . . . . . . . . . . 141

5.13 Control of $4 / r e v$ rotating frame blade load, $F_{z}$. Closed-loop control simulations: comparison between the baseline and controlled blade loads, $F_{z}$ obtained from the reduced-order model and the GENUVP/GAST simulations.142

5.14 Control of $4 / r e v$ rotating frame blade load, $F_{z}$. Closed-loop control simulations: comparison between the baseline and the controlled hub loads, $F_{Z}, M_{X}$ and $M_{Y}$ obtained from the reduced-order model and the GENUVP/GAST simulations. . . . . . . . . . . . . . . . . .

5.15 Control of $3 /$ rev rotating frame blade load, $M_{y} . H_{2}$ controller design performance and disturbance weights. . . . . . . . . . . . . . . . 145

5.16 Control of $3 /$ rev rotating frame blade load, $M_{y}$. Control command given to the TEF $\delta_{1}$ and $\delta_{3}$ by the $H_{2}$ controller. . . . . . . . . . . . 146

5.17 Control of $3 /$ rev rotating frame blade load, $M_{y}$. Closed-loop control simulations: comparison between the baseline and the controlled blade loads, $F_{z}$ and $M_{y}$ obtained from the reduced-order model and the GENUVP/GAST simulations. . . . . . . . . . . . . . . . . . 14 
5.18 Control of $3 /$ rev rotating frame blade load, $M_{y}$. Closed-loop control simulations: comparison between the baseline and controlled hub loads, $F_{Z}, M_{X}$ and $M_{Y}$ obtained from the reduced-order model and the GENUVP/GAST simulations. . . . . . . . . . . . . . . . . . . 148

5.19 Control of $4 / \mathrm{rev}$ rotating frame blade load, $F_{z}$. Control command given to the TEF $\delta_{1}$ and $\delta_{3}$ by the $H_{2}$ controller, advance ratio, $\mu=0.224$.

5.20 Control of $4 / r e v$ rotating frame blade load, $F_{z}$. Closed-loop control simulations: comparison between the baseline and controlled blade loads, $F_{z}$ obtained from the GENUVP/GAST simulations, at advance ratio, $\mu=0.224 .152$

5.21 Control of $4 /$ rev rotating frame blade load, $F_{z}$. Closed-loop control simulations: comparison between the baseline and controlled hub loads, $F_{Z}, M_{X}$ and $M_{Y}$ obtained from the GENUVP/GAST simulations, at advance ra-

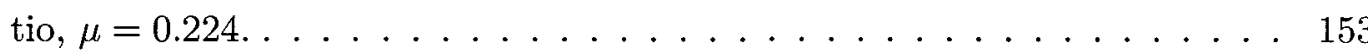

5.22 Control of $4 / r e v$ rotating frame blade load, $F_{z}$. Control command given to the TEF $\delta_{1}$ and $\delta_{3}$ by the $H_{2}$ controller, at advance ratio, $\mu=0.17 \ldots$. . .

5.23 Control of $4 / r e v$ rotating frame blade load, $F_{z}$. Closed-loop control simulations: comparison between the baseline and controlled blade loads, $F_{z}$ obtained from the GENUVP/GAST simulations, at advance ratio, $\mu=0.17$. 156

5.24 Control of $4 / r e v$ rotating frame blade load, $F_{z}$. Closed-loop control simulations: comparison between the baseline and controlled hub loads, $F_{Z}, M_{X}$ and $M_{Y}$ obtained from the GENUVP/GAST simulations, at advance ratio, $\mu=0.17 \ldots \ldots \ldots \ldots \ldots \ldots \ldots$ 


\section{List of Tables}

2.1 Summary of geometric properties of SHARCS blade. . . . . . . . . . . . 39

2.2 Eigenfrequencies at a rotational speed, $\Omega=162.8 \mathrm{rad} / \mathrm{s} \ldots \ldots . \ldots 39$

4.1 Sensitivity of TEF deflection on the blade loads and hub loads in terms of

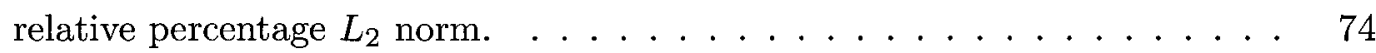

4.2 The relative percentage error norm $L_{2}$ between the blade loads obtained from LTI and LTP models $\left(n_{k}=12\right)$ and the GENUVP/GAST aeroelastic simulations under the physically realizable random TEF deflection of one blade. . . . . . . . . . . . . . . . . . . . . . .

4.3 Relative percentage error norm $L_{2}$ between the blade loads obtained from the LTP model of the One Blade Analysis with order $n_{k}=12$ and the GENUVP/GAST simulations when the TEF of one blade was deflected at the frequencies $1 /$ rev, $3 /$ rev and $5 /$ rev separately. . . . . . . . . .

4.4 Relative percentage error in amplitudes between the aeroelastic responses obtained from the system model and the GENUVP/GAST simulations at frequencies $1 / r e v, \cdots, 6 / r e v$, under the TEF excitation at frequencies $1 / r e v$, $3 / r e v$ and $5 / r e v \ldots \ldots \ldots \ldots \ldots \ldots \ldots \ldots \ldots \ldots \ldots \ldots \ldots \ldots \ldots \ldots$

4.5 The Relative percentage error norm $L_{2}$ between the blade loads obtained from the LTP models of the one blade analysis (1BA) with order $n_{k}=24$, and the all blades analysis (ABA) with order $n_{k}=48$, and the GENUVP/GAST aeroelastic simulations, when the TEF of all blades was deflected with a $\phi_{i}=90^{\circ}$ phase shift at $1 /$ rev frequency. . . . . . . . . . . . . . 101 
4.6 The relative percentage error in amplitudes between the blade loads obtained from the LTP models of the one blade analysis (1BA) with order $n_{k}=24$, and the all blades analysis (ABA) with order $n_{k}=48$, and the GENUVP/GAST simulations at the frequencies $1 / \mathrm{rev}, \cdots, 6 / \mathrm{rev}$, when the TEFs of all blades were deflected with a $\phi_{\imath}=90^{\circ}$ phase shift at $1 /$ rev frequency. . . . . . . .

4.7 The relative percentage error in amplitudes between the hub loads obtained from the LTP models of the one blade analysis (1BA) with order $n_{k}=24$, and the all blade analysis (ABA) with order $n_{k}=48$, and the GENUVP/GAST simulations at the frequencies $1 /$ rev $, \cdots, 6 / r e v$, when the TEFs of all blades were deflected with a $\phi_{\imath}=90^{\circ}$ phase shift at $1 /$ rev frequency. . . . . . . . 102

4.8 Relative percentage error in amplitudes between the blade loads obtained from the LTP models of the one blade analysis (1BA) with order $n_{k}=24$, and all blade analysis (ABA) with order $n_{k}=48$, and the GENUVP/GAST simulations at the frequencies $1 /$ rev, $\cdots, 6 / r e v$, when the TEFs of all blades were deflected with a phase shift $\phi_{i}$ at $3 /$ rev frequency. . . . . . . .

4.9 Relative percentage error in amplitudes between the hub loads obtained from the LTP models of the one blade analysis (1BA) with order $n_{k}=24$, and the all blade analysis (ABA) with order $n_{k}=48$, and the GENUVP/GAST simulations at the frequencies $1 / r e v, \cdots, 6 / r e v$, when the TEFs of all blades were deflected with a phase shift $\phi_{\imath}$ at $3 /$ rev frequency. . . . . . . . . . 108

4.10 Comparison of eigen frequencies of the SHARCS blade and the softened blade at a rotational speed of $\Omega=162.8 \mathrm{rad} / \mathrm{s} \ldots \ldots \ldots$ 


\section{List of Abbreviations}

1BA One Blade Analysis

ABA All Blades Analysis

ACF Actively Controlled Flap

ACSR Active Control of Structural Response

ACT Actively Controlled Tip

APL Active Pitch Link

ATR Active Twist Rotor

CFD Computational Fluid Dynamics

CSD Computational Structural Dynamics

DLR Deutsches Zentrum für Luft-und Raumfahrt, German Aerospace Center

EMP Exponentially Modulated Periodic

FEM Finite Element Method

GAST General Aerodynamic and Structural Tool

GENUVP GENeral Unsteady Vortex Particle

GP Geometrically Periodic

H Hold device

HHC Higher Harmonic Control

HTF Harmonic Transfer Function

xviii 
LFT Linear Fractional Transformation

LMI Linear Matrix Inequalities

LPV Linear Parameter Varying

LQG Linear Quadratic Gaussian

LQR Linear Quadratic Regulator

LTP Linear Time Invariant

LTP Linear Time Periodic

MOESP Multivariable Output-Error State-sPace

N4SID Numerical algorithms for Subspace State-Space System Identification

NTUA National Technical University of Athens

ONERA Office National D'Etudes et de Recherches Aerospatiales, French Aerospace Laboratory

PARMA Periodic Auto-Regressive Moving-Average

PTF Parametric Transfer Function

RFA Rational Function Approximation

S Sample device

SHARCS Smart Hybrid Active Rotor Control System

SIM Subspace Identification Methods

TEF Trailing Edge Flap

TFO Transfer Function Operator

VAM Variational-Asymptotic Method 


\title{
List of Symbols
}

\author{
Non-dimensional frequency $(\omega / \Omega)$ \\ $\alpha_{s} \quad$ Shaft angle \\ $\beta$ \\ Pumping amplitude \\ $\delta_{\imath} \quad$ Deflection of a trailing edge flap attached to the blade number $i$, positive \\ upwards
}

$\Delta_{\Psi}$

$\delta_{\Psi}$

$\Delta_{l k}$

$\Delta_{l_{s k}}$

$\zeta$

$\theta$

$\theta_{0}$

$\theta_{1 c}$

$\theta_{1 s}$

$\Theta_{w k}$

$\Theta_{s k}$
Azimuth angle separation in system identification simulations

Azimuth angle separation in aeroelastic simulations

Length of the vortex sheet originated from $k^{\text {th }}$ lifting body's trailing edge

Length of the vortex sheet originated from $k^{\text {th }}$ lifting body's separation point, respectively

Damping

Elastic deformation in torsional direction

Collective pitch angle

Lateral cyclic pitch angle

Longitudinal cyclic pitch angle

Angle of the vortex sheet originated from $k^{\text {th }}$ lifting body's trailing edge

Angle of the vortex sheet originated from $k^{\text {th }}$ lifting body's separation point 


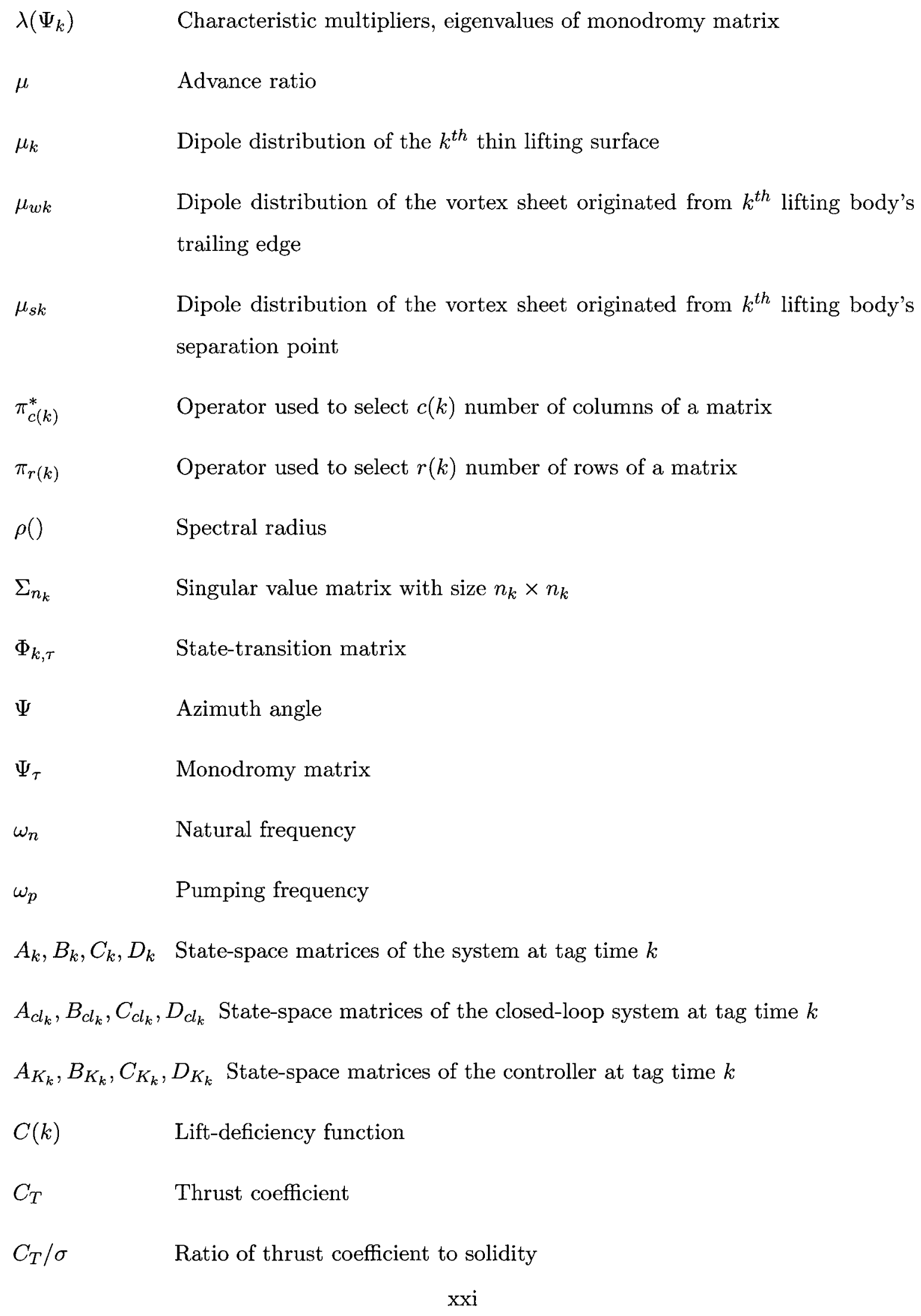




\begin{tabular}{|c|c|}
\hline $\mathcal{C}_{k}$ & Controllability matrix \\
\hline$F$ & Force \\
\hline$F_{L}()$ & Lower Linear Fractional Transformation \\
\hline$F_{x, \imath}$ & Rotating frame blade chord-wise load for blade number $i$ \\
\hline$F_{X}$ & Hub load in longitudinal direction \\
\hline$F_{y, \imath}$ & Rotating frame blade axial load for blade number $i$ \\
\hline$F_{Y}$ & Hub load in lateral direction \\
\hline$F_{z, 2}$ & Rotating frame blade vertical load for blade number $i$ \\
\hline$F_{Z}$ & Hub vertical load \\
\hline$g(t, \tau)$ & Impulse response function \\
\hline $\mathcal{G}$ & Harmonic Transfer Function \\
\hline$k$ & Sampled tag time index in one period \\
\hline$K_{k}$ & Controller system model at tag time $\mathbf{t}$ \\
\hline$K_{\infty_{k}}$ & $H_{\infty}$ Controller system model at tag time $\mathrm{t}$ \\
\hline$m$ & Number of inputs \\
\hline$M_{\jmath}\left(t_{k}\right)$ & Markov Parameters \\
\hline$M_{k}$ & System model in Linear Fractional Transformation form \\
\hline$m_{k}$ & Number of inputs at tag time $k$ \\
\hline$m_{\text {act }}$ & Number of inputs to the actuator weight \\
\hline$m_{d \imath s t}$ & Number of inputs to the disturbance weight \\
\hline$m_{n}$ & Number of inputs to the noise weight \\
\hline
\end{tabular}




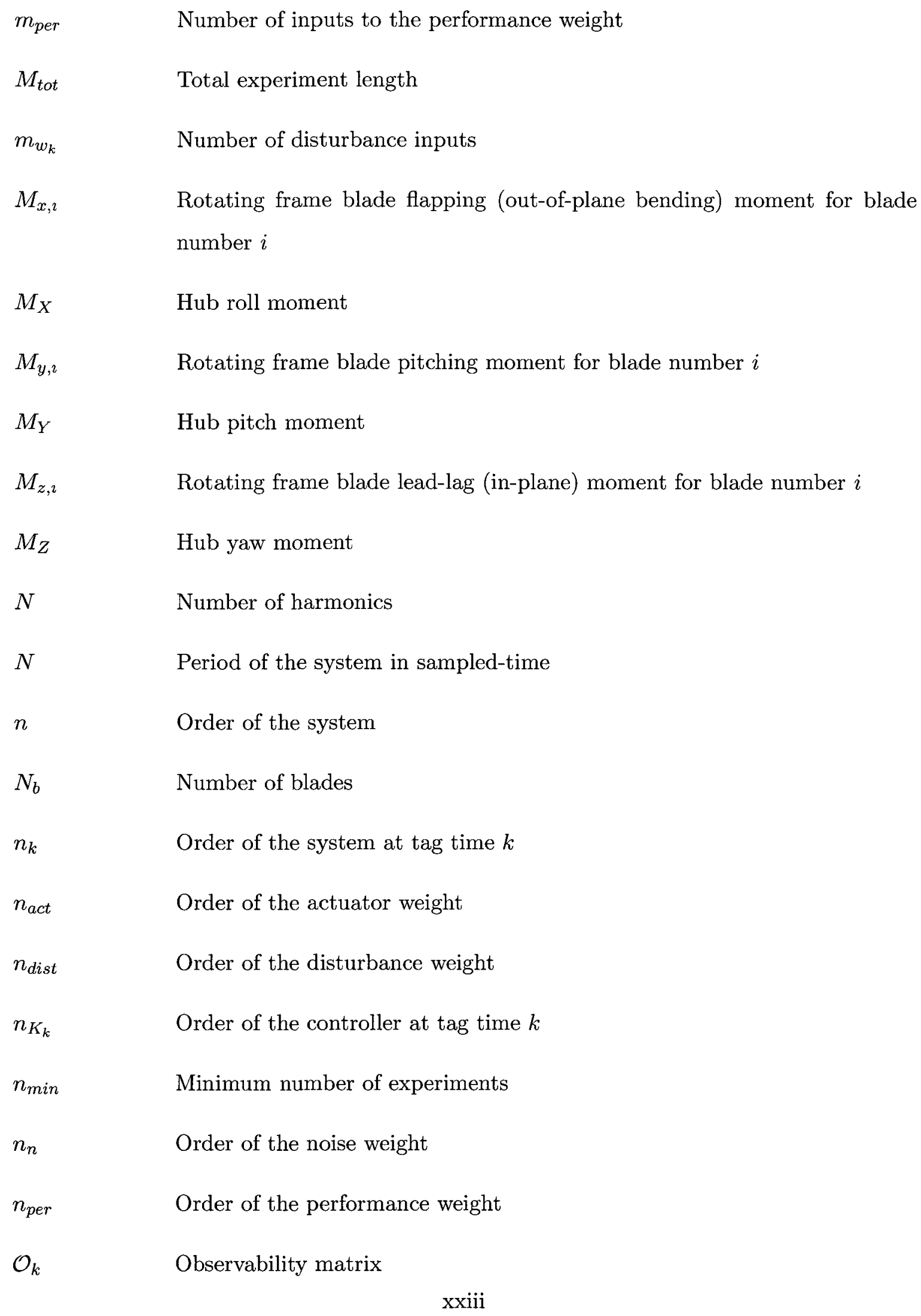




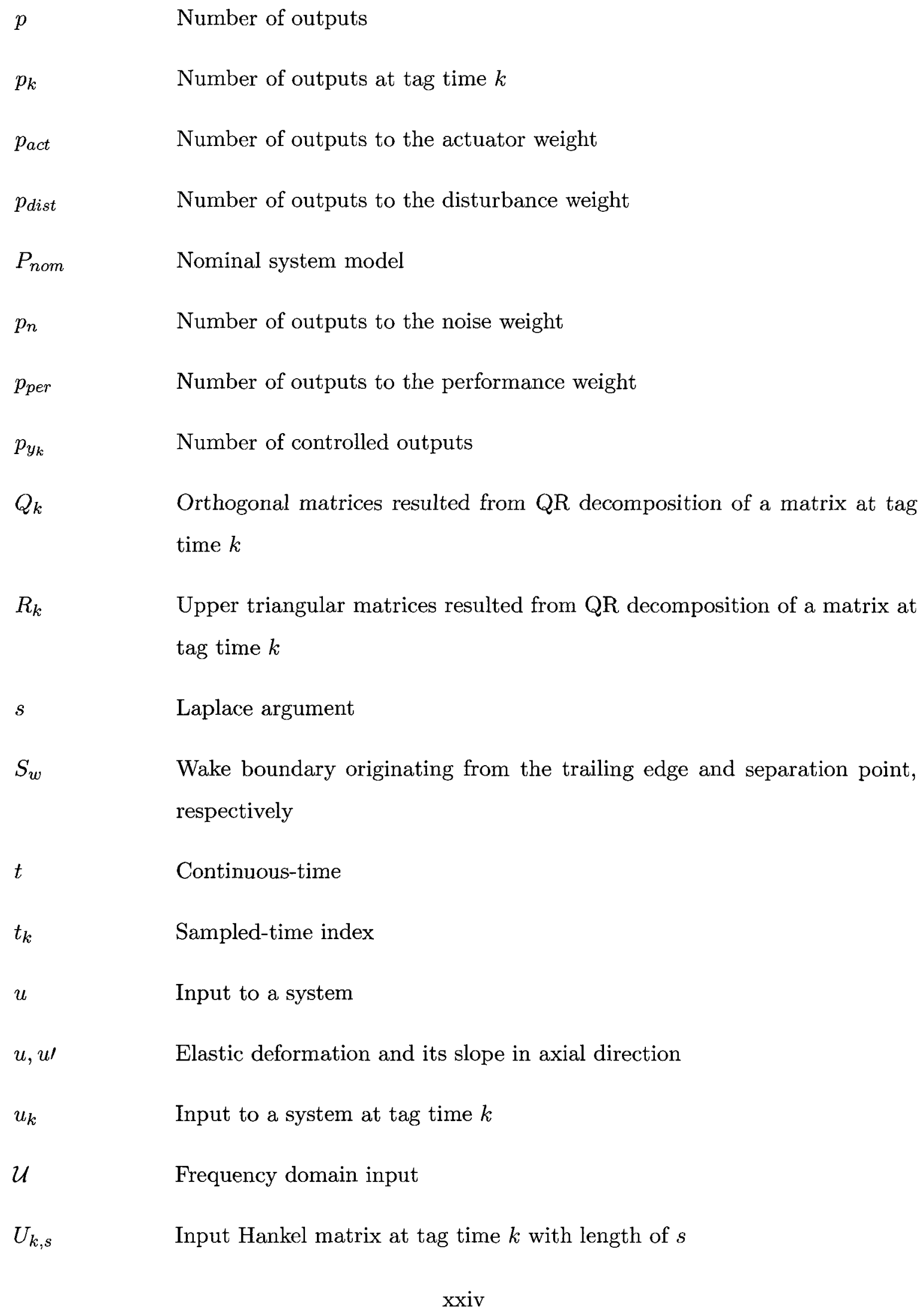




$\begin{array}{ll}v, v \prime & \text { Elastic deformation and its slope in cord-wise direction } \\ v_{k} & \text { Discrete-time signal } \\ \underline{v}_{p} & \text { Time-Lifted signal } \\ V_{k}\left(x_{k}\right) & \text { Time-varying Lyapunov's candidate function } \\ w & \text { Disturbance } \\ w, w \prime & \text { Elastic deformation and its slope in flapping direction } \\ W_{n} & \text { Weight for noise measure } \\ W_{a c t} & \text { Weight for actuation measure } \\ W_{d i s t} & \text { Weight for disturbance measure } \\ W_{p e r} & \text { Orequency domain output } \\ Y_{k, s} & \text { Weight for performance measure } \\ y_{k} & \text { State-vector variable } \\ x_{K} & \text { State vector for the controller at tag time } k \\ & \text { Output the system }\end{array}$




\section{Chapter 1}

\section{Sources of Helicopter Vibration and Means of Control}

Helicopters operate differently from fixed wing aircraft due to their hover and vertical take-off and landing capabilities. These unique capabilities make helicopters well-suited for tasks such as search and rescue and runway independent transportation. It is the multifunctional helicopter rotor, which can simultaneously provide thrust, propulsive force in forward flight, and attitude and position control of the helicopter, that contributes to its operational flexibility [1]. While performing all these tasks, helicopter rotor blades function within a highly complicated aerodynamic environment. In the forward flight regime in particular, aerodynamic complexity increases drastically as a result of near-blade and farblade aerodynamic phenomena. These two phenomena are schematically illustrated in Fig. 1.1. Near-blade aerodynamic phenomena include the effect of transonic flow with shock waves, which occur at the tip of the blade on the advancing side, and dynamic stall, originating from boundary layer separation, experienced by the blade on the retreating side. Far-blade aerodynamic phenomena are related to blade vortex interaction (BVI), which is caused by the interaction of tip vortices that emanates from the advancing blade with the upcoming blades for several revolutions. This far-field flow phenomena plays a very important role in helicopter blade aerodynamic loading $[1,2]$.

These aerodynamic phenomena result in fluctuating aerodynamic loads that, when coupled with the dynamic characteristics and elastic motion of the blade, create a challenging aeroelastic problem [2,3]. The main outcome of the aeroelastic problem is an exces- 


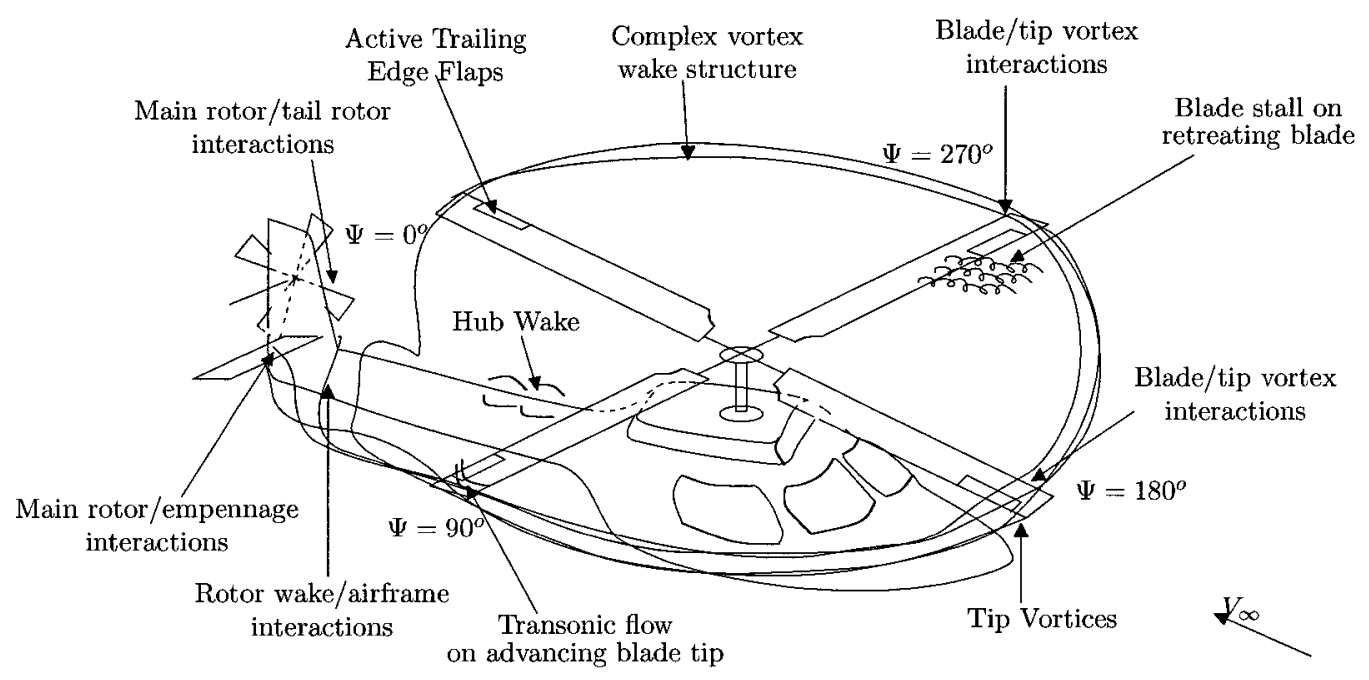

Figure 1.1: Schematic representation of aerodynamic environment around helicopter in forward-flight [1].

sive amount of noise and vibration transmitted to the fuselage through the rotor hub and shaft [2]. This excessive level of vibration degrades helicopter performance, pilot/passanger comfort and contributes to high cost maintenance problems.

The challenges of the helicopter vibration control problem are twofold. First, the development of control strategies for helicopter vibration suppression requires a coupled analysis of different research fields: aerodynamics, structural dynamics, and control theory. Second, since the helicopter blade experiences different flow phenomena at different azimuth angles, the aeroelastic problem is time-periodic, which adds mathematical complexity [2].

In the last three decades, several studies aimed at increasing helicopter performance from both an operational and economical point of view have been conducted [4]. Early attempts at helicopter vibration suppression are characterized by designs that include passive vibration absorbers and isolators attached to the helicopter frame. In this way, vibration was suppressed after it was transferred to the frame [4]. Although used in many operational helicopters, passive vibration suppression methods require undesirable weight additions and are generally ineffective at reducing the vibrations resulting from the complex unsteady aerodynamics of the rotor at different flight conditions [4]. In response to the high demand for reducing the helicopter vibration from industry, recent efforts have concentrated 
on developing active vibration suppression strategies [4]. These include Active Control of Structural Response (ACSR) [5-7], Classical Higher Harmonic Control (HHC) [8, 9], Individual Blade Control (IBC) with Active Pitch Link (APL) [10-12], Actively Controlled Flap (ACF) [13-18] and the Active Twist Rotor (ATR) [19-21].

Active vibration control requires three main steps: definition of the system, modeling of the system, and design and implementation of the controller. In order to develop a control strategy for helicopter vibration suppression by active means, efforts have traditionally followed a similar approach, here after referred to as the traditional approach, in completing these steps. In Fig. 1.2, the common features of the traditional approach are illustrated. In the traditional approach, the complex aerodynamic environment and the structural dynamics of the helicopter blade are independently reduced to a mathematically tractable model. The aeroelastic coupling between these models is developed and performed independently. Active control is implemented in the reduced aeroelastic model [9, 14, 15, 18, 22-24].

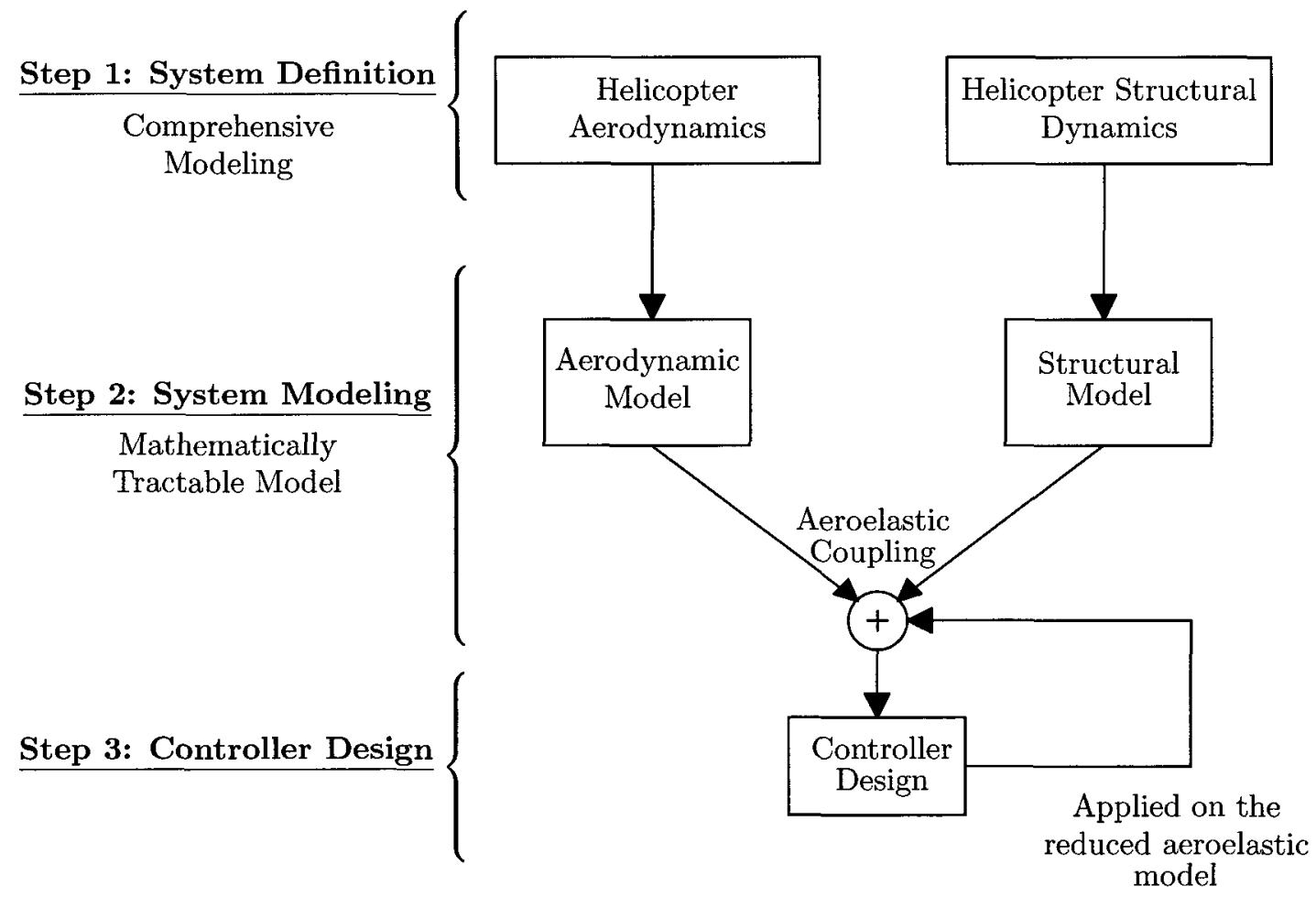

Figure 1.2: Traditional approach for active vibration control of helicopters. 
In this traditional approach, models of the rotor aerodynamics are derived from unsteady aerodynamic theory, and include predefined wake models. These models are indicial response function representations [1] and finite state approximations [25-27]. Alternatively, 2-D Computational Fluid Dynamics (CFD) simulations can be introduced through leastsquare fit of the aerodynamic response by rational function approximations [28]. The aerodynamic models used for controller design are essentially linear and the equations involve the time periodic velocity terms only $[9,24,29]$. Before aeroelastic coupling is performed, the unsteady aerodynamic models are further reduced to a quasi-steady model $[14,15,18,22,23]$. In addition, to model the structural deformations related to the out-of-plane bending, inplane bending, and elastic torsion of helicopter blade an Euler-Bernoulli beam model is commonly utilized. The beam model is discretized and Hamilton's principle is applied to obtain the equation of motion [14,22]. Displacements are expressed by Galerkin's method using the mode shapes of a rotating beam [24]. As a further simplification, only the rigid outof-plane bending of the elastic beam is considered when controller design is performed [15]. In summary, the traditional approaches have been using over-simplified aerodynamic and structural modes for controller design purposes.

Although this traditional approach is commonly utilized for vibration control applications, numerous experimental studies suggest that such oversimplified helicopter aerodynamics fail to predict actual helicopter blade loads [30]. The following references show that the assumptions of quasi-steadiness and the use of static, predefined wake models are erroneous [14,31-33]. For ACF applications in particular, the quasi-steady assumption was deemed invalid [30].

To this end, a novel active helicopter vibration control design methodology is proposed. The goal of the proposed approach is to start with a more accurate representation of the helicopter system, which would provide means of designing better active control systems still in the preliminary design phase of the project and potentially being directly employed in subsequent experiments. In the proposed approach, the system is defined by a coupled aeroelastic model that is obtained from comprehensive Computational Fluid Dynamics (CFD) and Computational Structural Dynamic (CSD) computer codes. Since coupled CFD/CSD aeroelastic simulations can only provide input/output relationships of 
the helicopter aeroelastic system, mathematically tractable models are directly obtained from detailed system identification. Very importantly, periodicity of the helicopter rotor aeroelastic response in the forward flight regime is fully modeled and no assumptions or simplifications are made. In addition, time-periodicity of the system is retained in the controller design stage and time-periodic controllers are designed. The use of a $H_{\infty}$ controller is proposed to prevent instability and performance degradation caused by the uncertainties in the system modeling. This proposed approach is depicted in Fig. 1.3.

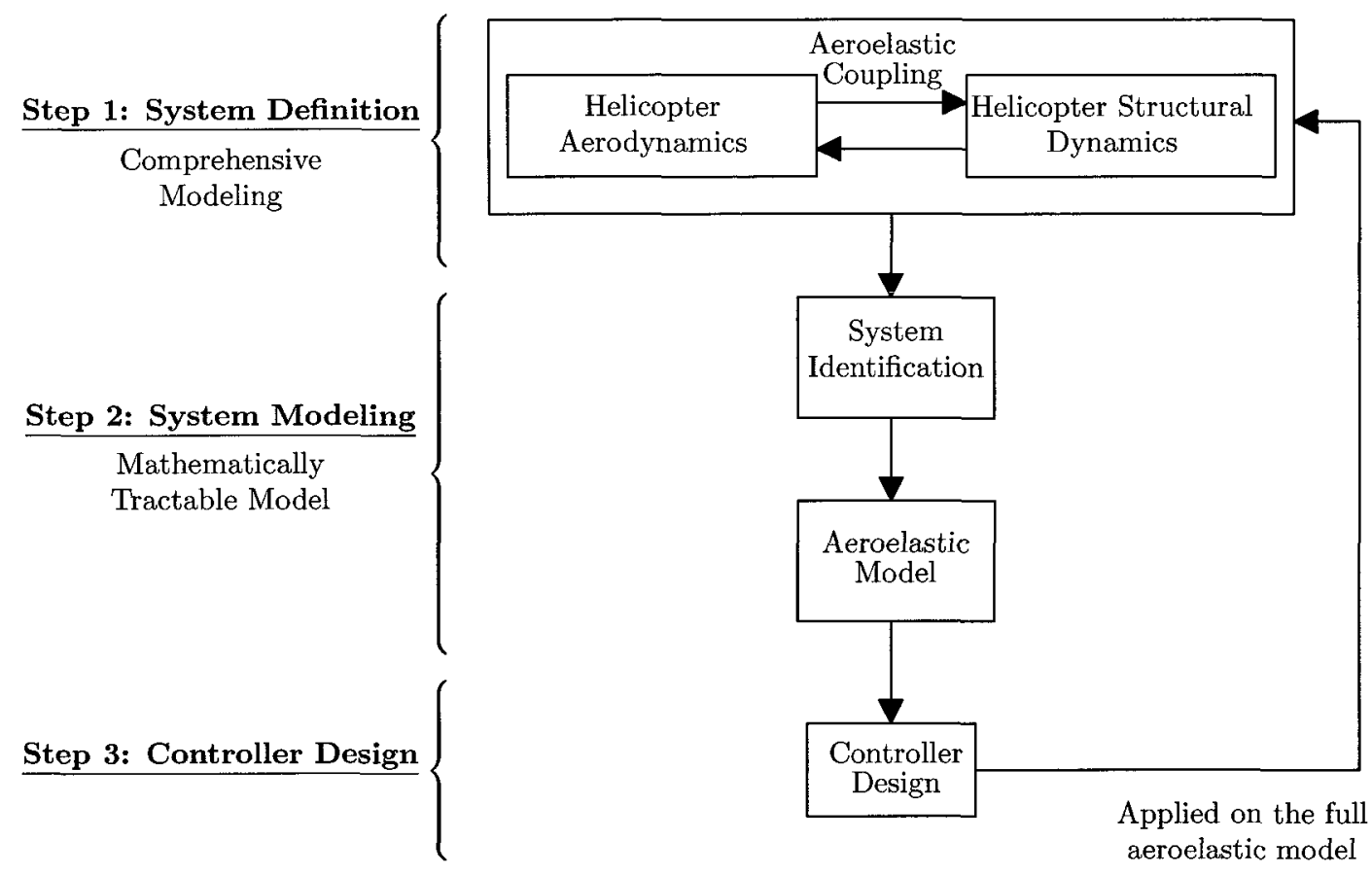

Figure 1.3: Proposed approach for active vibration control of helicopters.

The advantages of the proposed approach over the traditional approach are

- The aerodynamic and structural dynamics of the helicopter are modeled with intrinsically coupled CFD/CSD aeroelastic computer codes instead of using approximate analytical models.

- The aeroelastic coupling is performed before the model reduction instead of reducing the aerodynamic and the structural models independently. 
- True time periodicity of the aeroelastic motion is retained in the mathematical modeling.

- The proposed approach is directly transferrable to an experimental setting; i.e. on-line identification and real-time control.

We presented a summary of the causes of helicopter vibration and the commonly applied approaches for the helicopter vibration reduction, and introduced a novel approach to reduce the helicopter vibration and compared with previously applied traditional approaches. In the following sections, a literature review on the steps of the proposed approach, which includes aeroelastic modeling, time-periodic system modeling and control is presented. In Section 1.1, common helicopter aeroelastic modeling methodologies used for vibration reduction purposes are discussed. In Section 1.2, methods used to identify the helicopter's time periodic aerodynamic characteristics in a forward flight regime are introduced. In Section 1.3, controller design methods which are applicable to time-periodic systems are reviewed and summarized. In the final section of this chapter, previously applied controller design methods for helicopter vibration control applications are summarized.

\subsection{Overview of the Comprehensive and Approximate Mod- eling of Helicopter Aerodynamics and Structural Dy- namics}

An accurate modeling of both the aerodynamics and structural dynamics of the helicopter blade is necessary to achieve successful vibration control results in real-time implementations [28]. Although approximate aerodynamic models are crucial during initial stages because of their simplicity and ease of integration with controller design methods, the approximations lead to unexpected results in the implementation stages. As such, the final design of a controller for helicopter vibration suppression purposes should be conducted using higher fidelity aerodynamic and structural models [28]. For this purpose, comprehensive coupled CFD/CSD aeroelastic computer codes, in which the complex rotor aerodynamics and the rigid body and elastic deformations of the helicopter blade can be resolved more 
accurately, are paramount.

CFD methods vary significantly in terms of cost of computation and their ability to accurately model helicopter aerodynamics. Commonly used CFD methods for 3D helicopter applications model the aerodynamics using the Navier-Stokes equations [34], Euler equations and vortex particle methods [35]. A recent and detailed literature review on helicopter CFD methods is available [36]. Besides CFD methods, there are approximate analytical aerodynamic models that are used to express the unsteady aerodynamic loads of the helicopter rotor [1]. These approximate analytical methods have served as a basis for many controller design developments. The three most commonly referenced approximate aerodynamic models are indicial response function representation [1], finite state approximation [25-27] and rational function approximation (RFA) [28].

Development of approximate aerodynamic models was initiated with quasi-steady theory, in which wake effects on the aerodynamic force calculations were ignored [37]. To include the effect of shed vortices on the aerodynamic loads, Theodorsen introduced a liftdeficiency function, $C(k)$ [37]. In addition to the shed vortices, the effect of vortices that are present due to the preceding blades in aerodynamic lift and moment calculations were analyzed by Loewy [37]. Building on both Theodorsen's unsteady aerodynamic theory and Loewy's work, Leishman developed an approximate model in terms of indicial response function representations of the aerodynamic loads on the helicopter blades [1]. In his formulation, the effect of TEF deflections on the aerodynamic loads was also modeled. The validation studies of this approximate model were conducted by comparing the results of the model with the available experimental results [30]. For high Mach numbers and high oscillating frequency of TEFs, discrepancies between the approximate model and the experimental results were mentioned. It was also noted that the TEF hinge moment shows highly unsteady effects and, therefore, quasi-steady assumptions are invalid [30]. Peters developed a finite-state model for helicopter aerodynamic analysis [26]. His formulation accounts for the aerodynamic loads resulting from large translations and rotations and small elastic deformations of the helicopter blade. In addition, using an appropriate wake model (free or prescribed) the effect of the wake on the induced flow and the resultant aerodynamic loads is included in the finite state model. These aerodynamic loads are introduced 
as apparent mass, damping and stiffness matrices with finite dimensions. Although, the finite state approximation was considered to be very promising for helicopter applications, it cannot provide the same accuracy as compared to the CFD calculations for various flight regimes [26]. Another approximate model was obtained based on Rational Function Approximation (RFA) [28]. RFA was developed by approximating the aerodynamic loads in the frequency domain as rational functions using a least-squares fit to oscillatory response data. In [28], the simulations were conducted in an isolated 2-D airfoil; hence the model is not capable of representing the interaction of the blades specifically when the helicopter is flying in forward flight regime. In addition, the range of the oscillation amplitudes imposed on the airfoil may lead to errors when the blade experiences large amplitude oscillations.

In addition to CFD tools, CSD tools are needed to resolve the out-of-plane bending (flapping), in plane bending (lead-lag), torsional and axial deformations of the helicopter blade caused by unsteady aerodynamic loading and inertial loading. In the formulation of the kinematics, geometrical nonlinearities and initial twist must be included when the rotor blade is experiencing large deflections and rotations [38]. This formulation of the beam equations was first proposed by Hodges [39]. A more updated method is the VariationalAsymptotic Method (VAM), in which a 3-D geometrically nonlinear elastic blade is analyzed in two parts. First, from linear 2-D analysis, the cross-sectional properties of the blade are obtained. Next, a nonlinear 1-D analysis, using the cross-sectional properties derived in the first step, is used to solve for displacements, generalized strains and stresses $[38,40]$. However, for controller design purposes, the common approach is to discretize a Euler-Bernoulli beam model, express the displacements by Galerkin's method, and apply Hamilton's principle to obtain the equations of motion $[14,22,24]$.

As stated previously, this thesis introduces a new approach to active helicopter vibration suppression, summarized in Fig. 1.3. The proposed approach uses high fidelity aerodynamic and structural models. For an accurate representation of helicopter aeroelasticty, the GENeral Unsteady Vortex Particle (GENUVP)/ General Aerodynamic and Structural Tool (GAST) in-house aeroelastic CFD/CSD tool, developed in partnership by Carleton University and the Technical University of Athens, was used. The aerodynamic component of the code solves the complete flow field by coupling an indirect panel method with a vortex 
particle description of the wake. For viscous correction, an a posteriori correction scheme was applied to the loads using the ONERA model [35,41-44]. The structural components (helicopter blades, TEF, shaft, etc.) are modeled using a non-linear beam model formulated in second-order accuracy. The beam equation is discretized and solved using FEM methods. The dynamic and structural coupling of the flexible components is performed in the context of a multi-body analysis. Each flexible component is allowed to undergo rigid-body motions under kinematic and load constraints in addition to its elastic deformations. Aeroelastic coupling in the GENUVP/GAST code is performed by transferring the aerodynamic loads of a particular aerodynamic element to the corresponding structural element as an externally applied force. The resultant elastic deformations are then transferred back to the corresponding aerodynamic element by deforming the aerodynamic mesh [35,41-44]. More detailed information on the GENUVP/GAST aeroelastic code is provided in Section 2.2.

\subsection{Modeling and System Identification of Time-Periodic Sys- tems}

This section provides an overview of time-periodic systems, specifically Linear Time Periodic (LTP) systems. A historical development on the input/output maps and system identification methods of LTP systems is included.

\subsubsection{Brief Introduction of LTP Systems and Input/Output Mapping}

The first detailed theory on LTP systems dates back to the 1870's when Mathieu conducted research on the natural modes of vibration of lakes with elliptic boundaries [45]. Shorty after, Floquet introduced the well known Floquet Theory that serves as a basis for stability and analysis of LTP systems [45].

Time-periodic systems have been studied in many engineering problems $[45,46]$. In signal processing and control applications, the system undergoes computational operations that force the system to become time-periodic. For example, in sampled-data systems, where the sampling is performed with different rates $[47,48]$, continuous-time systems are controlled with discrete-time controllers $[49,50]$, and Linear Time Invariant (LTI) systems are 
controlled with periodic controllers. As a result, the controlled system shows time-periodic behavior [51]. In addition, when nonlinear systems are linearized along time periodic trajectories, the resultant linear system becomes LTP. Two examples of such phenomenon are satellite altitude control [52], and control of inverter locomotives [53]. On the other hand, in some engineering problems the actual physics of the system is TP. Examples of naturally TP systems are helicopter rotor systems in forward flight [3], electrical and thermal diffusion systems with elliptical boundary conditions, and diffraction of acoustic and electromagnetic waves around ellipsoidal objects [45].

Mathieu's equation (Eq. 1.1) is a simple TP system used as a representative model for general TP system behavior. In this equation $w_{p}$ and $\beta$ are the pumping frequency and pumping amplitude or amplitude of parametric excitation, respectively.

$$
\ddot{x}(t)+2 \zeta \omega_{n} \dot{x}(t)+\omega_{n}^{2}\left[a-2 \beta \cos \left(\omega_{p} t\right)\right] x(t)=\frac{F(t)}{m}
$$

In the case where $\beta=0$, Mathieu's equation reduces to a time-invariant system. Figure 1.4 gives the comparison of the steady state output responses of the Mathieu equation when $\beta=0$ (time-invariant) and when $\beta \neq 0$ (time-periodic).
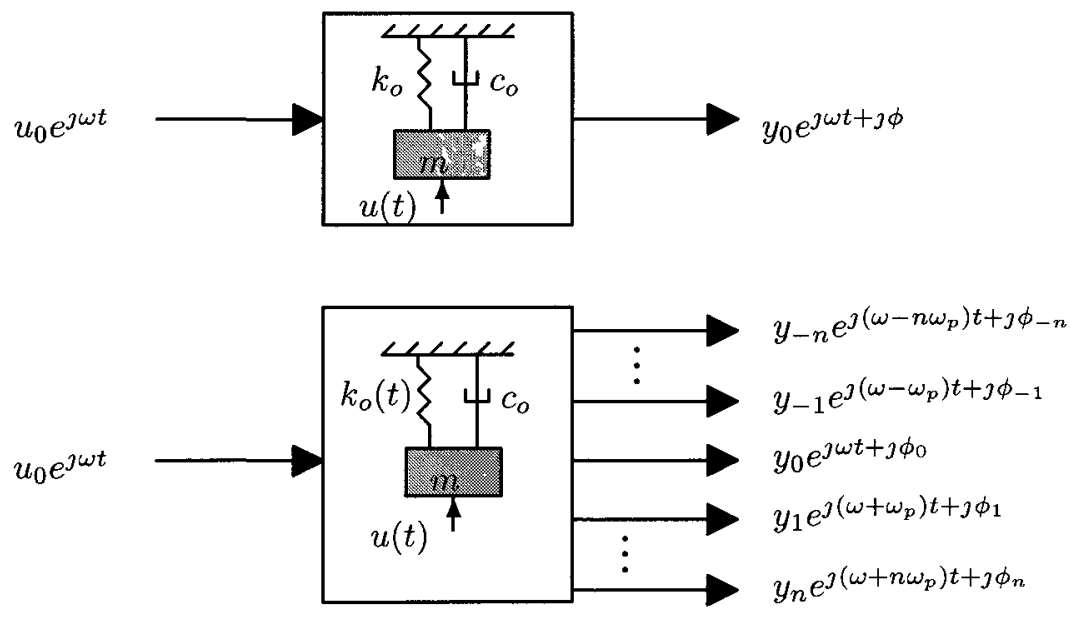

Figure 1.4: Comparison of input-output relations for LTI and LTP systems.

Although the steady-state output of an LTI system that is driven by a complex exponential input has the same frequency content $(\omega)$ with a different amplitude and phase, an 
LTP system's output includes not only the forcing frequency. $\omega$, but also higher harmonics of frequency $\omega \pm n \omega_{p}$ at amplitudes and phases $y_{n}$ and $\phi_{n}, n=0, \pm 1, \cdots, \infty$, respectively. This frequency response behavior of LTP systems shows that the complex exponential signal $u(t)=e^{\jmath w t}$ cannot provide a simple input/output operator. This result was observed by both Floquet and Hill, who suggested the use of Geometrically Periodic (GP) and complex Exponentially Modulated Periodic (EMP) signals as test signals for LTP systems [54].

In the time domain, the response of an LTP system to an impulsive input is observed to be dependent on the time that the input is applied. This result shows that the impulse response function of an LTP system is time-dependent, $g(t, \tau)$ and that, if the input/output map is needed, a single impulse response function cannot provide enough information on the system's characteristics [46].

Different methods have been introduced to provide an input/output operator map for LTP systems. These methods are based on the knowledge of either the time-dependent impulse response function, and/or the state-space model of the system under consideration $[48,55,56]$. Three different, commonly referenced operator maps that are obtained using impulse response functions and state-space models are: the Parametric Transfer Function, the Harmonic Transfer Function and the Transfer Function Operator. Relationships between these input/output maps are given in [57].

The Parametric Transfer Function (PTF) was introduced for the analysis of continuoustime, time-varying systems by Zadeh [55]. His formulation can be found in [58]. PTF was derived from the convolution integral of the time varying impulse response function $g(t, \tau)$ and the input $e^{\jmath w t}$, and the resultant transfer function has both frequency and time dependency, $H(\jmath \omega, t)$.

The Harmonıc Transfer Function (HTF) was introduced by Wereley and Hall, specifically for helicopter applications [56]. HTF maps EMP input space to EMP output space using the Fourier coefficients of the continuous-time impulse response matrix, $g(t, \tau)$ or state-space matrices. HTF is an infinite dimensional transfer function operator that has no time dependency. An equivalent operator was introduced independently by Nitzsche in the Laplace domain by shifting the Laplace argument $(s)$ along the imaginary axis by an integer multiple of harmonics [59]. Although HTF serves as a good framework for theoret- 
ical analysis, its infinite dimension causes problems for practical implementations. Later studies investigated the criteria for truncation of infinite dimensional HTF based on the continuous-time, time-varying impulse response function behavior, i.e., roll-off of higher frequencies in terms of Markov Parameters [60]. For sampled-data systems, where there is a periodic control in the loop, and for discrete-time systems, it was shown that the HTF has a finite dimension $[15,61]$. For discrete-time systems, an equivalent expression to the HTF can be obtained by taking the $z$-transform of the discrete-time state-space matrices [48]. The mapping between the EMP input/output spaces with the HTF is given in Eq. 1.2. In this equation, $\mathcal{G}_{0}(s)$ gives the transfer function between the input at frequency $\omega$ to the output at frequency at $\omega$, and $\mathcal{G}_{ \pm 1}(s)$ give the transfer functions between the inputs at $\omega \mp \omega_{p}$ to the output at $\omega$.

$$
\left[\begin{array}{c}
\vdots \\
\mathcal{Y}\left(s-j \omega_{p}\right) \\
\mathcal{Y}(s) \\
\mathcal{Y}\left(s+j \omega_{p}\right) \\
\vdots
\end{array}\right]=\left[\begin{array}{ccccc}
\ddots & \vdots & \vdots & \vdots & . \cdot \\
\ldots & \mathcal{G}_{0}\left(s-j \omega_{p}\right) & \mathcal{G}_{-1}\left(s-j \omega_{p}\right) & \mathcal{G}_{-2}\left(s-j \omega_{p}\right) & \ldots \\
\ldots & \mathcal{G}_{1}(s) & \mathcal{G}_{0}(s) & \mathcal{G}_{-1}(s) & \ldots \\
\cdots & \mathcal{G}_{2}\left(s+j \omega_{p}\right) & \mathcal{G}_{1}\left(s+j \omega_{p}\right) & \mathcal{G}_{0}\left(s+j \omega_{p}\right) & \ldots \\
. & \vdots & \vdots & \vdots & \ddots
\end{array}\right]\left[\begin{array}{c}
\vdots \\
\mathcal{U}\left(s-j \omega_{p}\right) \\
\mathcal{U}(s) \\
\mathcal{U}\left(s+j \omega_{p}\right) \\
\vdots
\end{array}\right]
$$

For a system of order $n\left(x \in \mathbb{R}^{n}\right)$, with $m$ number inputs $\left(u \in \mathbb{R}^{m}\right)$ and $p$ number of outputs $\left(y \in \mathbb{R}^{p}\right)$, the transfer function matrix has dimension $\mathcal{G}_{0}(s) \in \mathbb{C}^{m \times p}$ and the resultant $\mathrm{HTF}$ has dimension $\mathcal{G} \in \mathbb{C}^{m(2 N+1) \times p(2 N+1)}$, where $\mathrm{N}$ is the number of harmonics included. The Transfer Function Operator (TFO) was obtained for discrete-time systems by applying $z$-transform to a time-lifted formulation [48]. Time-lifting has been commonly used in sampled data systems, which include both continuous and discrete-time systems in the loop. A simplified sampled data system is given in the block diagram representation in Fig. 1.5, where the continuous-time signals are shown as solid lines and the discrete-time signals are shown as dotted lines. $S$ and $H$ are sample and hold devices, and $K_{d}$ represents the discrete-time controller. The base period, $h$ is divided into $N$ intervals, and the discrete time signal, $v_{k}$ is referred to the subperiod $\frac{h}{N}$ of the base period $h$. The lifted signal, $\underline{v}_{p}$ is 

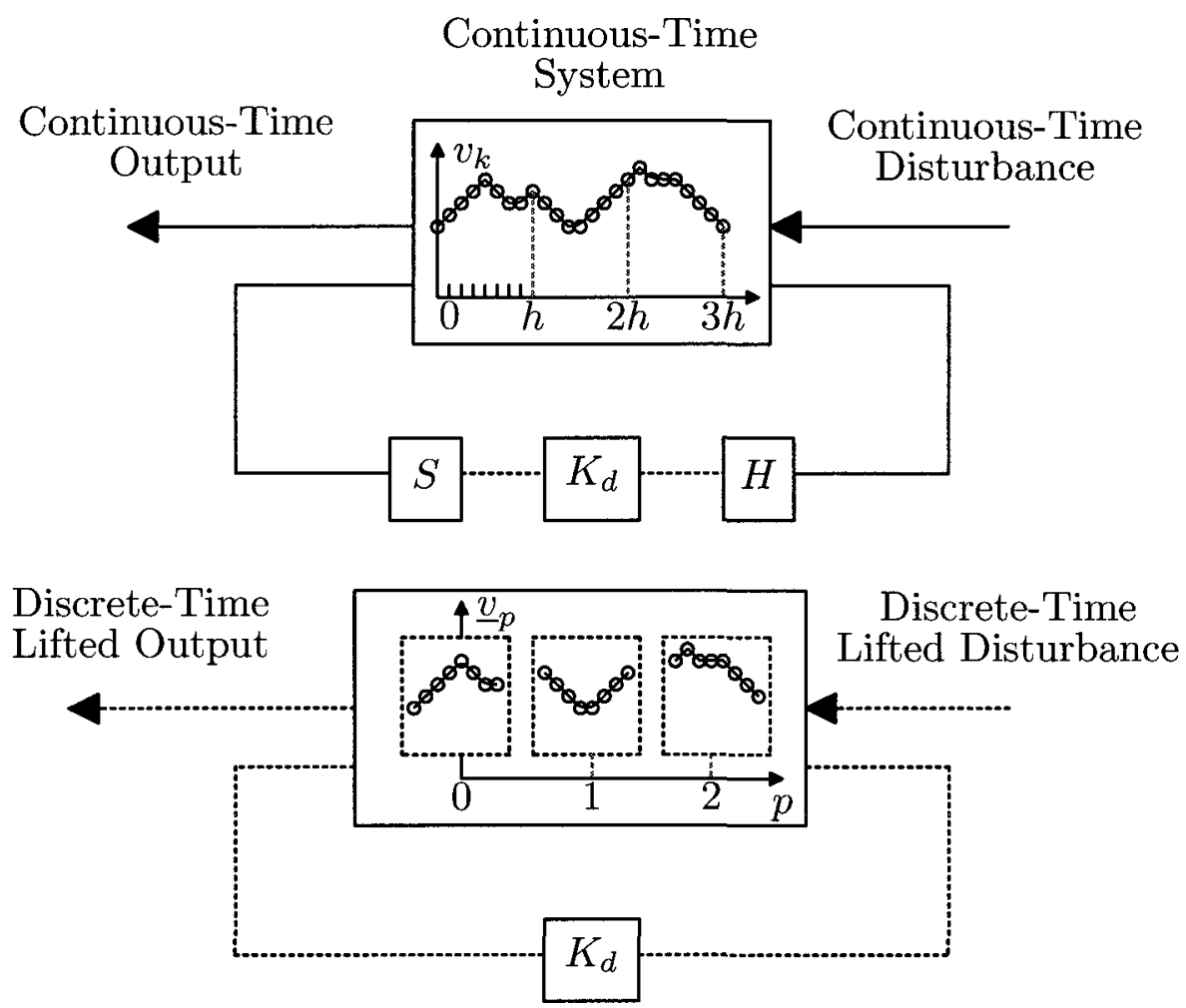

Figure 1.5: Time-lifting of continuous time signals [50,62].

expressed by packing all subperiod signals, as shown in Eq. 1.3.

$$
\underline{v}_{p}=\left\{\left[\begin{array}{c}
v_{0} \\
v_{1} \\
\vdots \\
v_{N-1}
\end{array}\right],\left[\begin{array}{c}
v_{N} \\
v_{N+1} \\
\vdots \\
v_{2 N-1}
\end{array}\right], \cdots,\left[\begin{array}{c}
v_{p h} \\
v_{p h+1} \\
\vdots \\
v_{(p+1) h-1}
\end{array}\right]\right\}
$$

In an analogous way, time-lifting is applied to LTP systems for which the base period, $h$ is the period of the LTP system $(h=T)$, and $\frac{h}{n}$ is the sampling period of the discretetime signal. Both the input and output spaces are lifted, as shown in Eq. 1.3, and the system's states are expressed in time-invariant form [63]. In this formulation, the lifted state-space equation has a time-invariant dynamic matrix but the input and output matrices become larger in dimension. This result is analogous to a result of the Floquet theory for continuous-time TP systems. For frequency-domain analysis, the Transfer Function 
Operator is obtained by taking a $z$-transform of the lifted state-space matrices. For a system of order $n\left(x \in \mathbb{R}^{n}\right)$, with $m$ number of inputs $\left(u \in \mathbb{R}^{m}\right)$ and $p$ number of outputs

$\left(y \in \mathbb{R}^{p}\right)$, the resultant time-lifted state-space matrices have dimensions $F \in \mathbb{R}^{n \times n}, G \in$ $\mathbb{R}^{n \times N m}, H \in \mathbb{R}^{N p \times n}$, and $E \in \mathbb{R}^{N p \times N m}$.

As mentioned previously, all methods listed above assume that the time-varying impulse response function, $g(t, \tau)$, or state-space model matrices are available either in the continuous or in the discrete-time domains. However, when the system's response is given as input/output pairs only, system identification becomes necessary to obtain the impulse response functions or the state-space matrices.

\subsubsection{Historical Development of Identification of Time Periodic Systems}

System identification is a methodology by which a system model is selected based on an input/output data set [64]. Modern system identification methods date back to 1965 when two papers were introduced to the system identification community [65]. The first paper, by Åström and Bohlin, was based on the Maximum Likelihood method for the parameter estimation of input-output models [65]. The second paper, by Ho and Kalman, gave the first solution to state-space identification (i.e realization) and laid of the foundations for stochastic realization, and much later for the subspace identification methods $[65,66]$. These two papers introduced the idea of looking at the system identification problem as a design problem [64]. This design problem aims at obtaining an approximate model of the system depending on its application [64]. System identification methods have been developed under these directions since then, and were applied to LTP systems with slight modifications.

System identification has three design elements: an input/output data set, a model set, and a criterion to select the best model [64]. To obtain an informative input/output data set for the system identification, a priori knowledge on the characteristics of the system is required to design the experiment (or the simulation). By using the input/output data set, the model set is iteratively updated until it satisfies the defined criterion. Model sets can be classified as non-parametric and parametric. Non-parametric models include impulse responses, frequency responses, spectral density functions, etc. Parametric models include transfer functions, state-space models, and time series that are written in terms of a finite 
number of parameters [64].

Non-parametric system identification of the HTF was first implemented by applying time shifted sine-sweep signals to the swash-plate [67]. The algorithm given in [67] assumes that for the identification of $N$ harmonics $2 N+1$ time-shifted sine-sweep input signals should be given to the system. On the other hand, assuming that $N$ number of harmonics contribute to the system response leads to errors in the system identification, and results in an over-estimation of the included harmonics. This result was also observed by Nitzsche [59]. The impulse response characteristics should have been analyzed first to determine the highfrequency roll-off characteristics. It was later shown by Hwang that the sine-sweep signal is not an adequate test signal for the LTP systems for the identification of the HTF [68]. Instead, he suggested a method based on spectral theory and applied it to the modeling of rigid out-of-plane motion of the helicopter blade. Using optimization, parameters of the equation of motion, such as the advance ratio, were searched to obtain a best fit for the HTF. This parameter search could be applicable for systems with lower dimensions, however, with increasing system complexity, computational costs increase significantly and render the approach inefficient.

In parametric system identification, when the parameter-based model is defined in terms of difference equations, the parameters are estimated to minimize the prediction error using recursive methods. If the parameter-based model is defined using state-space matrices, recursive estimation using periodic Kalman filter, state-space realization methods or subspace identification methods is necessary. Although all these methods are equally capable of identifying parametric models, only the subspace identification method offers the added advantage of not requiring precise a priori estimation of the systems time variation and order [69].

The difference equation for discrete-time representation of LTP systems is expressed using the Periodic Auto-Regressive Moving-Average (PARMA) model (see Eq. 1.4). The AR part contains the periodic parameters, $a_{r}(k)$, which relate the previous outputs to the output at time $k$, whereas the MA part contains the periodic parameters, $b_{s}(k)$, which relate 
the effect of previous inputs to the output at time $k$.

$$
\begin{aligned}
y(k)= & a_{1}(k) y(k-1)+a_{2}(k) y(k-2)+\cdots+a_{r}(k) y(k-r) & \text { [AR part] } \\
& +b_{0}(k) u(k)+b_{1}(k) u(k-1)+\cdots+b_{s}(k) u(k-s) & \text { [MA part] }
\end{aligned}
$$

where $a_{\imath}(k)=a_{\imath}(k+N) \in \mathbb{R}^{p}$ and $b_{\jmath}(k)=b_{\jmath}(k+N) \in \mathbb{R}^{m}$

For the estimation of these periodic parameters, three different approaches have been historically considered. In the first approach, the PARMA model was written in a time-lifted form, and then maximum-likelihood, least-squares. or pseudo-linear regression algorithms were applied to estimate the parameters recursively $[48,61]$. In the second approach, each periodic parameter of the PARMA model was rewritten as a summation of an $N$ number of harmonics, as given in Eq. 1.5, which reduces the problem to a recursive estimation of the constant parameters [70].

$$
\begin{aligned}
y(k) & =\sum_{n=0}^{N} a_{1_{n}} e^{\jmath n w t} y(k-1)+\cdots+\sum_{n=0}^{N} a_{r_{n}} e^{\jmath n w t} y(k-r) \\
& +\sum_{n=0}^{N} b_{0_{n}} e^{\jmath n w t} u(k)+\cdots+\sum_{n=0^{N}} b_{s_{n}} e^{\jmath n w t} u(k-s)
\end{aligned}
$$

where $a_{\imath_{n}} \in \mathbb{R}^{p}$ and $b_{\jmath_{n}} \in \mathbb{R}^{m}$. In the third approach, the PARMA model was written in state-space form and the parameters were estimated using the periodic Kalman filter [71,72].

Minimal state-space realization was first introduced by Ho and Kalman and was based on the sequence of Markov Parameters (impulse response) of a discrete-time deterministic LTI system. Markov Parameters were used to construct the Hankel matrix that was then decomposed into the observability and controllability matrices to obtain the state-space matrices. The method was adapted to LTP systems and time-lifted state-space realizations of LTP systems were obtained by Lin and King [73]. This realization method was only applicable for fixed-dimension LTP systems, and the resultant state-space realization was not minimal. An extension of this method provides state-space realization of lifted-systems with time-varying dimension [74]. However, since time-lifted state-space models have higher input-output matrix dimensions and cause time delays in the control implementations, they are not very favorable [75]. In addition, Varga [76] identified numerical stability problems of time-lifted systems caused by the non-orthogonal transformations. These conclusions lead researchers to seek robust methods of realizing periodic state-space matrices. Both Wang 
and Varga proposed a method to calculate periodic state-space realization from the timelifted state-space matrices with a time-varying dimension $[75,76]$. Once the time-periodic state-space matrices are obtained, a cyclic form, introduced by Flamm [77], is used to obtain the time-invariant representation. However, this form also causes the system to have higher dimensions and, therefore, for computational purposes is not effective [76].

Whereas these state-space realization methods are applicable for discrete-time LTP systems, Sandberg introduced a method for state-space realizations of continuous-time LTP systems [78]. The state-space matrices were expanded in Floquet-Fourier form and then the input and output relation matrices were written as a sum of Fourier coefficients. For the systems having sufficiently smooth impulse responses, the method provides a statespace realization. Unfortunately, the method given in [78] also suffers for not providing time-periodic state space matrices at each time step.

The Subspace Identification Method (SIM) is an extension of standard state-space realization methods used for the identification of deterministic systems whose input-output data are corrupted by noise yielding a combined deterministic-stochastic system. SIM does not require impulse responses to identify the state-space matrices; only input/output pairs (with some constraints) are used to identify the state-space matrices. In addition, SIM does not require precise a priori knowledge of the model's structure. Starting from the state-space realization algorithm of Ho and Kalman, state-space matrices are obtained by imposing a constraint to their dimension to approximate the system's impulse response [79]. After this initial work, studies focused on developing new algorithms to provide state-space matrices under the noisy input/output data set, without the use of stochastic information of the data. The most widely referenced algorithms are intersection algorithms [80], the Multivariable Output-Error State-sPace (MOESP) algorithm [69], and the numerical algorithms for subspace state-space system identification (N4SID) [81]. A detailed history of the development of subspace identification algorithms for discrete-time LTI systems is given in [81]. Inspired by these developments, researchers adapted SIM algorithms to timeperiodic systems. Time-periodic state-space matrices were first identified from time-lifted state-space matrices [82]. Later algorithms, such as MOESP, used ensemble input/output pairs to directly identify the periodic state-space matrices [69]. Detailed information on the 
MOESP algorithm for LTP systems is presented in Section 3.4

In summary, system identification is now recognized as a design problem where the choice of model set, experimental design (or simulation), identification method and identification algorithm all depend on the specific problem at hand. If the system model is identified for controller design purposes, the capability of the model and its limitations should be clearly stated to ensure stability of the closed-loop system. For LTP systems, time-invariant representations are commonly utilized (HTF in continuous-time, time-lifted form in discrete-time). While in HTF the frequencies are lifted at integer multiples of the pumping frequency, $\omega_{p}$. In time-lifted state-space representations, the input/output spaces are lifted in time. For closed-loop control applications, where the controller is designed based on the HTF, delays are created when the Fourier transform of the output within one full period is taken. If the controller is designed based on the time-lifted state-space model, it is then necessary to wait one full period to sample all the information and calculate the control signal to be applied in the next period. As a result, a preferable approach is to obtain the periodic state-space matrices at each time step in one period, rather than identifying time-lifted state-space matrices that represent the behavior of the system in one full period. In addition, identification methods are available in both continuous and discrete-time; during the controller design stage, the conversion between the described two time representations might be necessary.

\subsubsection{LTP Modeling of Helicopter Rotor Response}

Experimental HTF identification of a helicopter rotor response was first carried out by Shin [21] using $2 N+1$ shifted sine-sweep excitations in the twist degree of freedom. However, during further analysis the authors decided to ignore the time periodicity of the system and model the dynamics as LTI. This conclusion was likely a result of the incorrect initial assumption that only $N=1$ harmonics are present in the output. Spectral-based system identification was first performed by Hwang [68] to model the rigid out-of-plane motion of the helicopter blade with HTF. The identification method was combined with an optimal search algorithm to obtain the parameters of the equation of motion. Unfortunately, as pointed out by the author, this parameter search becomes very inefficient for systems of 
higher order and/or complexity. Time-lifting formulations for HHC control applications were first carried out by Colaneri [83] to identify helicopter blade out-of-bending response. Later, a more detailed aeroelastic motion of the helicopter rotor system was considered, and the time-lifting formulation was used to express time-invariant helicopter dynamics for vibration control purposes with periodic HHC [9].

For helicopter vibration control purposes, the PARMA model identification was carried out by Roget [14] to model the transfer function of a TEF. The parameters of the transfer function were estimated on-line using the Kalman filter over one full rotation. One disadvantage of the PARMA model is the fact that precise information on the model is required. If the system model is not defined accurately, the estimation of the time-periodic parameters often requires many iterations to minimize the output error. For example, in the experimental study Roget [14] estimates the transfer function parameters from TEF deflections using between 40 - 160 iterations, where each iteration requires 8 blade revolutions. The long time required to compute the parameters causes significant computational cost when high-fidelity computer simulations (CFD) are used to model the helicopter rotor aerodynamics.

In the past, researchers have also used Subspace Identification Methods (SIM) for the identification of helicopter rotor dynamics for vibration suppression $[84,85]$. Helicopter rotor dynamics was modeled as a Linear Parameter Varying (LPV) system where the parameters vary periodically [85]. However, in this model, very simplified aerodynamic and structural properties were used. The SIM was also used for the wind turbine applications, where the parameter variation of the wind turbine dynamics was introduced and modeled as time-periodic [86] and time-varying [87]. In both of these studies, several Monte Carlo simulations were carried out to obtain the LPV state-space matrices. Since SIM does not require a precise a priori knowledge of the model structure, as the PARMA model does, it is in general considered to be a better approach for the identification of time-varying, complex dynamical systems [69]. 


\subsection{Controller Synthesis for LTP Systems}

Controllers modify the dynamics of the system under consideration in order to meet certain objectives and specifications. One common and fundamental objective is to stabilize an unstable system by including a controller either in open-loop or closed-loop (feedback). However, controllers alter the dynamic characteristics of stable systems [88]. These characteristics are defined in terms of location of poles, desired loop-shapes and norm-based performance functions [88].

Controllers are designed based on the system model to be controlled as well as the uncertainty model that adequately captures the essential features of the plant and its environment. The uncertainty may appear in different forms, as disturbances and noise (environment and signal uncertainty), or as imperfections in the system model (model uncertainty). For a defined system model, i.e. for a given set of systems, $M$ and a set of exogenous inputs, $w$ designing an implementable controller so that the feedback system satisfies the given specifications, $z$ defines a controller synthesis problem [89]. The general feedback diagram for the controller synthesis problem is shown in Fig. 1.6. In this diagram the system $M$ includes the plant model and the filters (weighting functions) that are used to shape the disturbance and noise in the plant's environment. $\Delta$ shows the uncertainty in the system model and $K$ is the controller.

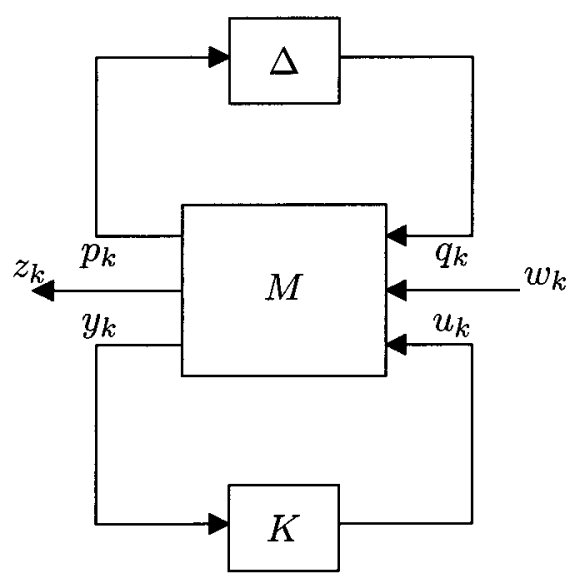

Figure 1.6: General feedback diagram for controller synthesis problem [89]. 
There are many controller design methods that account for the variations in the plant model and its environment. Examples are gain scheduling, adaptive controllers, robust controllers and their combinations. These three methods are well developed and several textbooks are available [50,88-91]. In gain scheduling, the control parameters are adjusted in an open-loop manner depending on the switching condition [90,92]. In the adaptive control method, the controller parameters are adjusted based on adaptation rules and, in general, parametric type system models are required [90]. In robust control, sets of system models are defined and the controllers are designed to satisfy the stability and performance criteria for all previously defined sets of models [50,88, 89, 91]

$H_{\infty}$ is a robust controller synthesis problem, in which the performance criteria is defined based on the infinite norm of the transfer function between the disturbance and error signal [89]. The $H_{\infty}$ control design method was first introduced in the 1980's to overcome the robustness issues of Linear Quadratic Gaussian (LQG) controllers (special form of $H_{2}$ controller) [93]. Since then, their research has focused on both the formulation of the $H_{\infty}$ synthesis problem and its solution. The Matlab Robust Control toolbox was developed for both continuous-time and discrete-time LTI systems [94]. There are currently three methods available to solve the optimal controller synthesis problem with respect to both $H_{2}$ and $H_{\infty}$ norms. The first is based on state-space models using Riccati Equations [88, 89,91]. The second is a polynomial approach that requires system models represented by polynomial matrices [95]. Finally, the third method is based on an optimization problem using Linear Matrix Inequalities (LMI) as constraints [96,97]. More detailed information on the conditions, formulations and solutions of $H_{2}$ and $H_{\infty}$ control synthesis problem are available $[48,50,52,98-100]$.

$H_{\infty}$ controllers provide very satisfactory results specifically in real-time aerospace applications when there is an uncertainty in the plant and its environment. Ulker et al. $[101,102]$ showed in the wind tunnel experiments to study fin buffet alleviation that superior vibration suppression levels can be obtained by using the $H_{\infty}$ controller when compared to the LQGtype controllers over different inflow speeds. In addition, while the LQG controller created instabilities in the closed-loop system with the increasing inflow speed, the $H_{\infty}$ controller was robust to changes in the damping and in the stiffness of this aeroelastic system. 
In real-time implementations, the plant's environment is in the continuous-time domain, and the control signal is calculated using sampled sensor information. For these feedback systems, controllers can either be designed in an indirect or direct way $[50,103]$. In the indirect way, either the continuous-time controllers are designed for the continuous-time plant model and then discretized with a given sample rate, or the plant model is first discretized and then the discrete-time controllers are designed for this discrete-time plant model. In the direct way, the discrete-time controllers are designed for the continuous-time plant model [50]. Figure 1.7 shows the different possible configurations of the controller design problem [103]. Depending on the plant model either the direct or indirect way can be used.

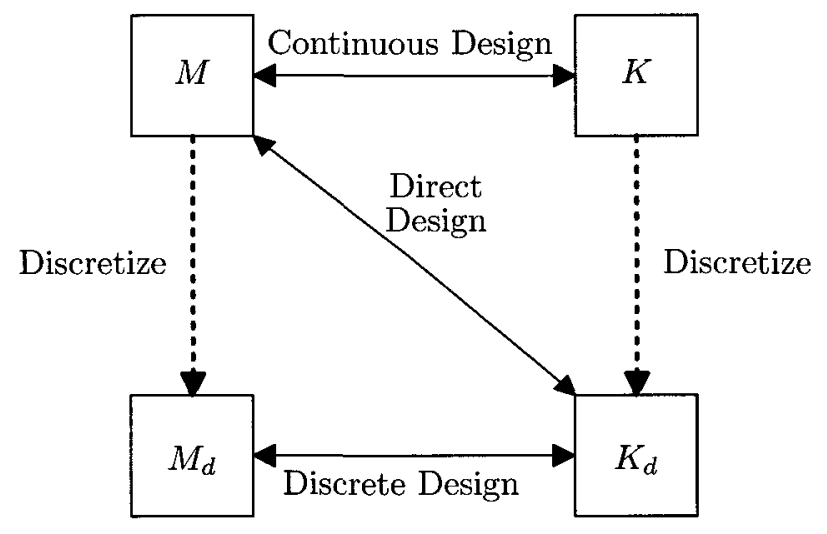

Figure 1.7: Controller synthesis; direct versus indirect way [103].

Controller synthesis for discrete-time LTP systems has been focussed in the research of multirate sampled-data systems, which are LTP systems. In Fig. 1.7, the direct design approach results in a LTP system, and the controller design for these systems has been performed by first lifting the continuous-time state-space model into a LTI discrete-time state-space model. Then, discrete-time $\mathrm{H}_{2}$ (LQG) or $H_{\infty}$ formulations and their respective solutions are applied on this discrete-time LTI system.

Based on the above two different representations of the time-periodic system, two different controller synthesis formulations have been considered. In the first formulation, the time-lifted state-space formulation was used to express the continuous-time system as 
a discrete-time system and a Ricatti-based solution was proposed for the controller design [50]. The resultant controller was time-periodic but the controller state-space matrices were also in time-lifted form. In the implementation of time-lifted controllers, it is necessary to wait for a full period to be completed, which results in time delays. In the second formulation, the time-periodic state-space matrices were given in cyclic form [77] and the $H_{2}$ (LQG) and $H_{\infty}$ controllers were designed either using LMIs [88,100,104,105] or solving the time periodic Riccati Equations [106-111]. The resultant controller was obtained to be time-periodic and the state-space matrices of the controller are available at each time along the period.

The solution of control problem using LMIs does not require additional software. Once the control problem is formulated, the Matlab Robust Control toolbox can be used to solve the time periodic controller parameters. However, the solution of discrete-time periodic Riccati Equation requires new algorithms [106]. Recently, a Periodic Systems Toolbox was developed for Matlab [109]. Yet it is not still available for public use.

\subsubsection{Literature Survey on Controller Design For Helicopter Vibration Suppression}

Helicopter vibration control studies have predominately used $T$-matrix algorithms for Higher Harmonic Control (HHC) [112]. The T-matrix is the sensitivity matrix between the control input from the rotor swashplate (or Trailing Edge Flaps) and the output measured in terms of blade and hub loads. The control action includes the adaptive estimation or identification of the $T$-matrix. The estimation of the $T$-matrix has been carried out using the Kalman filter or recursive least-squares for either online or off-line applications in the vicinity of baseline conditions (i.e local), or yet for the full-flight envelope (i.e. global) [112]. Since the steady $T$-matrix was shown to be equivalent to the time-invariant part of HTF (Eq. 1.2), frequency-domain system identification techniques were also used in $T$-matrix identification [56]. The fundamental assumption of the $T$-matrix algorithm is that the helicopter dynamics can be modeled as Linear Time Invariant (LTI). It also assumes that the response of a helicopter to higher harmonic inputs is quasi-steady [9]. Controller design based on the estimated or identified $T$-matrix is usually carried out by minimizing the 
quadratic cost function to regulate the helicopter vibratory hub loads. Linear Quadratic Regulators (LQR) and LQGs are the commonly used controller design techniques for helicopter vibration control $[9,24,56]$. Both design techniques depend heavily on the accurate estimation or identification of the $T$-matrix. In the implementation of the controller there is a wait time between control updates to allow the transformation from time domain to frequency domain, and to allow dissipation of a transient response. Furthermore, after the control signal is applied, the signal is kept constant during the entire interval, which is generally taken as one revolution or one quarter of the revolution for four-bladed rotors. Recently, Periodic HHC (PHHC) control using the T-matrix has been extended and now includes the periodicity [9]. In this latter study, static feedback LQR controllers were designed based on the time-lifted LTP system.

$T$-matrix based optimal controller has been designed for helicopter vibration control using TEFs where the quadratic cost was defined to minimize both the TEF deflection and the hub loads [113]. The static controller coefficients were based on both the estimated $T$-matrix and the cost function weights. Other research focused on the design of LQG controllers based on a LTP transfer function from the solution of the continuous-time periodic Ricatti equations for the control of vibration caused by dynamic stall using TEFs [24].

Adaptive controller design was also used for helicopter vibration control using TEFs [14, 114]. On-line identification of the transfer function from individual TEF deflections was carried out recursively using Kalman filter estimators. A deterministic controller was designed based on the model by minimizing the linear quadratic cost function. The effectiveness of the controller was validated by wind tunnel tests resulting in vibration suppression levels.

Neural-network based controllers were also utilized to control helicopter vibration using TEFs to overcome the modeling errors in the aeroelasticity of the helicopter rotor sytems [23]. Neural-network based controllers do not require a priori knowledge of the disturbances in the helicopter environment. Hence, it is not necessary to identify the disturbances to design the controller. In this specific application, the TEF deflection was adaptively controlled to reduce the blade loads without performing system identification. The controllers were developed both for hover and forward flight regimes and closed-loop computer simulations were performed. These closed-loop simulations produced promising 
results for helicopter vibration control. However, in neural network control applications, because the system identification was not carried out, on-line controller design was necessary, which lengthens the experiment (or simulation) time.

$H_{\infty}$-based controllers have been designed for helicopter vibration control using an actively controlled pitch link. A continuous time state-space model was obtained from aeroelastic computer simulations $[115,116]$. In forward flight, time-periodic state-space matrices were obtained by first performing the Fourier transform of the time-varying state-space matrices, and then reducing the order of state-space matrices based on their singular values. Time-invariant $H_{\infty}$ controllers were designed based on this reduced model, and for the forward-flight case, the gain scheduling method was applied on the time-invariant controllers at the specific azimuth angle $[115,116]$.

Controller design for helicopters is an attractive subject for systems and control engineers because the helicopter's TP behavior in forward flight has been well documented by simplified aerodynamic (quasi-steady flow) and structural dynamic equations (mainly the rigid body motions) [3]. Based on these simplified mathematical models, LQ controllers were designed along with Kalman state estimators for several flight conditions [117]. Then, using the same model, the flight conditions (i.e. the advance ratios) were modeled as parameter variations and the TP Linear Parameter Varying system approach was used to model the helicopter response under higher harmonic excitations of the swash plate. For vibration suppression of the helicopter blade, $H_{\infty}$ controllers were designed based on the TP Linear Parameter Varying (LPV) system model and closed-loop simulations were shown to be very successful [118].

\subsection{Proposed Active Vibration Control Validation Case}

For the purpose of validating the proposed approach, active vibration of the Smart Hybrid Active Rotor Control System (SHARCS) blade has been attempted (see Fig. 1.3). SHARCS is an international project lead by the Carleton University Rotorcraft Research Group [16]. The hybrid control concept introduced by the SHARCS project uses an Active Pitch Link (APL), an Actively Controlled Flap (ACF), and an Actively Controlled Tip (ACT) simul- 
taneously to control both the structural and flow properties of the helicopter blade system. For structural control, the conventional pitch link is replaced with the APL that changes the blade's modal frequencies and/or damping [119]. For flow control, the effective camber of the helicopter blade is modified by actively controlling the deflection of the TEF. In addition, the strength and the location of vortices at the blade's tip are altered by the ACT to control Blade Vortex Interaction that generates noise and vibration. Figure 1.8 illustrates the SHARCS blade with three individual control means.

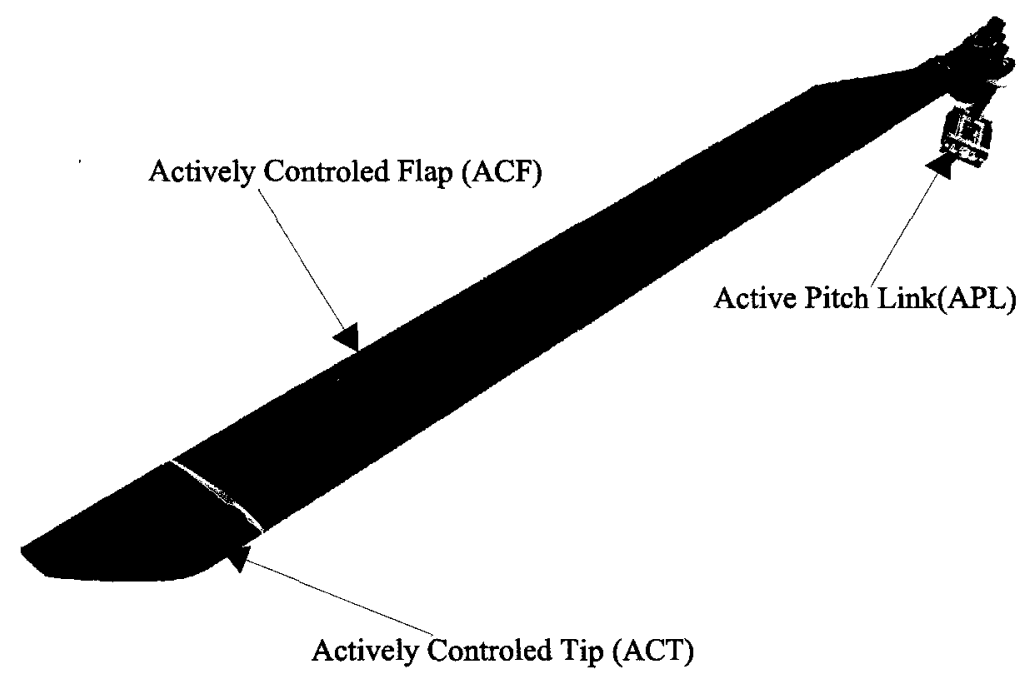

Figure 1.8: The SHARCS blade with three individual control means [16].

There are three main reasons for using the SHARCS blade in the validation analysis. First, all the necessary structural and mechanical properties of the SHARCS blade are freely and readily available from the Carleton University Rotorcraft Research Group. Second, the SHARCS blade was equipped with novel actuation mechanisms that allow more flexibility on the actuator capability. Third, the ultimate goal of the SHARCS project is to perform the wind tunnel validation tests.

To actively control the vibratory hub loads, only the TEF is used in this research. The numerical simulations were performed using a comprehensive in-house aeroelastic code, GENUVP/GAST, using the SHARCS blade structural and mechanical properties. The system identification was carried out to obtain the effect of the Trailing Edge Flap (TEF) on the helicopter blade loads around a pre-defined baseline condition defined by a fixed 
advance ratio and trimmed swash plate settings.

\subsection{Thesis Objectives}

The objectives of this thesis are in summary to;

1. demonstrate the applicability of the developed theory for the experimental system identification of coupled aeroelastic response of helicopter rotor system in forward flight regime;

2. identify a discrete-time Linear Time Periodic state-space model of aeroelastic response of helicopter rotor system caused by the deflection of the trailing edge flap from measurements that are obtained from high fidelity coupled CFD/CSD aeroelastic computer codes;

3. design a discrete-time Linear Time Periodic $H_{\infty}$ controller for the suppression of the blade loads using optimization methods with LMIs;

4. analyze the stability of the discrete-time Linear Time Periodic helicopter rotor system when the controller is connected in feedback and validate the performance of the $H_{\infty}$ controller, which is designed based on the model, by implementing it in the high fidelity coupled CFD/CSD aeroelastic simulations;

5. design a discrete-time Linear Time Periodic $\mathrm{H}_{2}$ controller for the suppression of the blade loads and hub loads by solving time-periodic discrete-time Riccati Equations.

6. validate the stability and performance of the $H_{2}$ controller by implementing it in the high fidelity coupled CFD/CSD aeroelastic simulations;

\subsection{Main Contributions}

The main contributions of this research for the state-of-the-art knowledge in the field of developing helicopter active control systems for the suppression of vibration are as follows: 
1. First application of system identification to obtain a discrete-time Linear Time Periodic state-space model from comprehensive coupled CFD/CSD aeroelastic tools. Compared to previous applications, where overly-simplified aerodynamic models were utilized, coupled CFD/CSD aeroelastic computer simulations provide higher-fidelity approximations of true helicopter rotor aeroelasticity.

2. First design and numerical simulation of a Linear Time Periodic $H_{\infty}$ and $H_{2}$ controller that does not create time delays (other than time-lifting applied for $15^{\circ}$ azimuth angle) in control applications and that can be directly applied in experimental setups.

3. Development of in-house solver for discrete-time Riccati Equations to design $\mathrm{H}_{2}$ controllers.

\subsection{Organization of the Thesis}

Chapter 1 presents a brief background on the modeling of aeroelastic response of helicopters for control applications. A detailed background on system modeling, system identification and controller design methods for Linear Time Periodic systems is presented. The SHARCS project, which was taken as the test case for all the simulations, is introduced. The significance of the thesis, objectives and the main contributions are given in the present chapter.

In Chapter 2, the comprehensive coupled CFD/CSD aeroelastic code is introduced. The properties of the SHARCS blade are given and the selected baseline simulation results are summarized.

In Chapter 3, the applied theory on the system identification of the Linear Time Periodic systems from noisy measurements is presented. The formulation of discrete-time Linear Time Periodic $H_{\infty}$ and $H_{2}$ controller synthesis problems and their solution methods are described step-by-step.

In Chapter 4, the application of the theory presented in Chapter 3 on the identification of the helicopters aeroelastic response caused by the TEF deflection is presented. First, the assumptions of linearity and periodicity are validated. Then, the helicopter rotor system's aeroelastic model is obtained in discrete-time LTP state-space form and validated using simulation results obtained from the aeroelastic code. 
Chapter 5 discuss the selection of the $H_{\infty}$ and $H_{2}$ controller design parameters and formulation of the block diagram for the helicopter vibration control purposes. The optimal control TEF deflections and the closed-loop control simulation results, obtained from reduced-order model and GENUVP/GAST aeroelastic simulations are presented in this chapter.

Chapter 6 concludes by summarizing the results and recommendations for future work. 


\section{Chapter 2}

\section{Aeroelastic Code and Simulations}

\section{$2.1 \quad$ Introduction}

In this chapter, the in-house aeroelastic computer code is introduced. Aerodynamic modeling of near-blade and far-field phenomena, structural modeling of flexible components and coupling between the aerodynamic and structural results are briefly explained. Properties of the SHARCS blade and the simulation results at the selected flight regime are summarized.

\subsection{Aeroelastic Code}

The GENeral Unsteady Vortex Particle (GENUVP)/ General Aerodynamic and Structural Tool (GAST) has been extensively used by Carleton University RotorCraft Research Group for the helicopter rotor system aeroelastic/aeroacustic time domain simulations for the vibration reduction purposes. It was first developed at the National Technical University of Athens (NTUA) and then modified for further improvements. The code was validated in comparison with experimental data by NTUA for different flight regimes [35,41-44] and the validations of the code incorporating the trailing edge flap (TEF) is still under investigation.

The aerodynamic component of the code, the GENUVP is a panel method code utilizing a vortex particle wake model for calculating the flow field around multi-component configurations. For the viscous correction, an a posteriori correction scheme was applied to the loads based on the ONERA model. Both attached and separated flows can be resolved by the GENUVP/GAST. The basis of GENUVP code is the Helmholtz decomposition in 
which the flow field around a rotorcraft is decomposed into its irrotational and rotational components. The irrotational component is due to the presence of bluff bodies in the proximity to the rotor, while the rotational component is due to the vortices that are on the lifting surface and shed by the lifting surfaces. The thick lifting surfaces are described by a combination of source and vorticity distribution whereas the thin lifting surfaces are described by only dipole distributions. The vortices shed by the airfoil are given as straight vortex sheets when they are shed for the first time. Subsequently the vorticity that the sheet segment carries is integrated to form a vortex particle and it is convected in the flow field.

The detailed 2-D and 3-D modeling of the blade and its wake, and the formation of the vortex particles are given in Fig. 2.1 and Fig. 2.2, respectively. In Fig. 2.1, $S_{w}$ shows the wake boundary originating from the trailing edge and $S_{s}$ shows the wake boundary due to separation. The location of the separation point is obtained from the viscous flow properties. $\mu_{k}, \mu_{w k}$ and $\mu_{s k}$ are the dipole distributions of the $k^{t h}$ thin lifting surface, and the vortex sheets that are originated from the $k^{\text {th }}$ lifting body's trailing edge and separation point respectively. The dipole distributions are considered to be piece-wise constant for the wake and for the bound vortex, $\mu_{k}$; two constant value distributions are imposed starting from the leading edge to the separation point and from the separation point until the trailing edge.

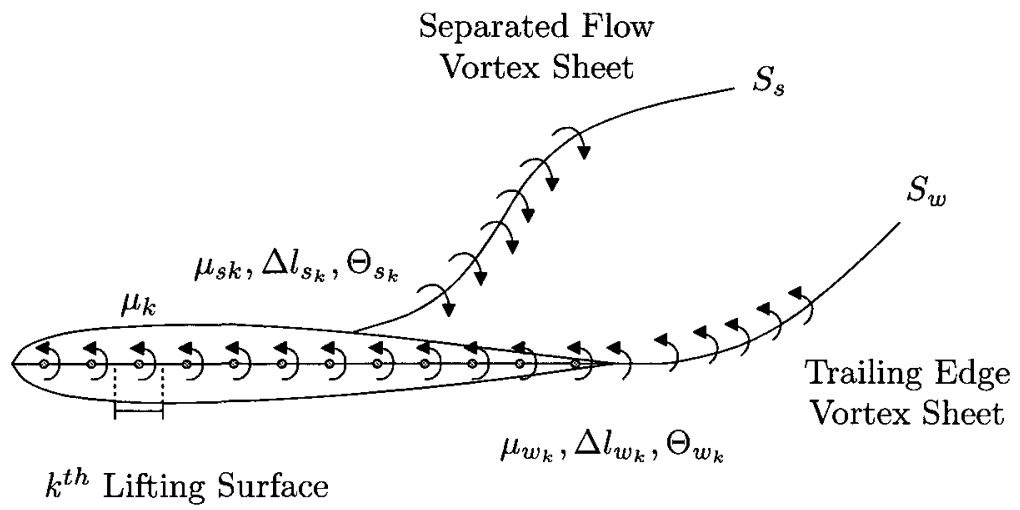

Figure 2.1: Representation of airfoil and its wake. 
Due to the unsteadiness of the flow, the unknown distributions $\mu_{k}, \mu_{w k}$ and $\mu_{s k}$ corresponding to $k^{t h}$ lifting surface vary with time. In addition, since the vortex sheets $S_{w_{k}}, S_{s_{k}}$ are freely moving material surfaces, the geometry of the problem, i.e. the length $\left(\Delta l_{w_{k}}, \Delta l_{s_{k}}\right)$ and the angle $\left(\Theta_{w_{k}}, \Theta_{s_{k}}\right)$ of the vortex sheets, is also time dependent. In order to solve for these unknown flow parameters two types of conditions are imposed. The first one is the kinematic condition: the non-entry condition on all the solid surfaces, and the condition that describes the material motion of the vortex sheets. The second one is the dynamic condition: the zero pressure jump throughout the vortex sheets.

The vortex sheet $S_{w}$ is shed at each time step from so called vorticity emission line, which is the trailing edge of lifting body and possibly its tip edges. The tip edge emission occurs in the case of blade tip vortices and the gap between the trailing edge flap and blade. Once the vortex sheet is shed, the surface vorticity of the sheet is integrated to create a vortex particle as shown in Fig. 2.2. Therefore, vortex sheet assumption is retained only for the near region of every wake, and then rest is transformed into free spatial vorticity (a vortex particle approximation is introduced here). 


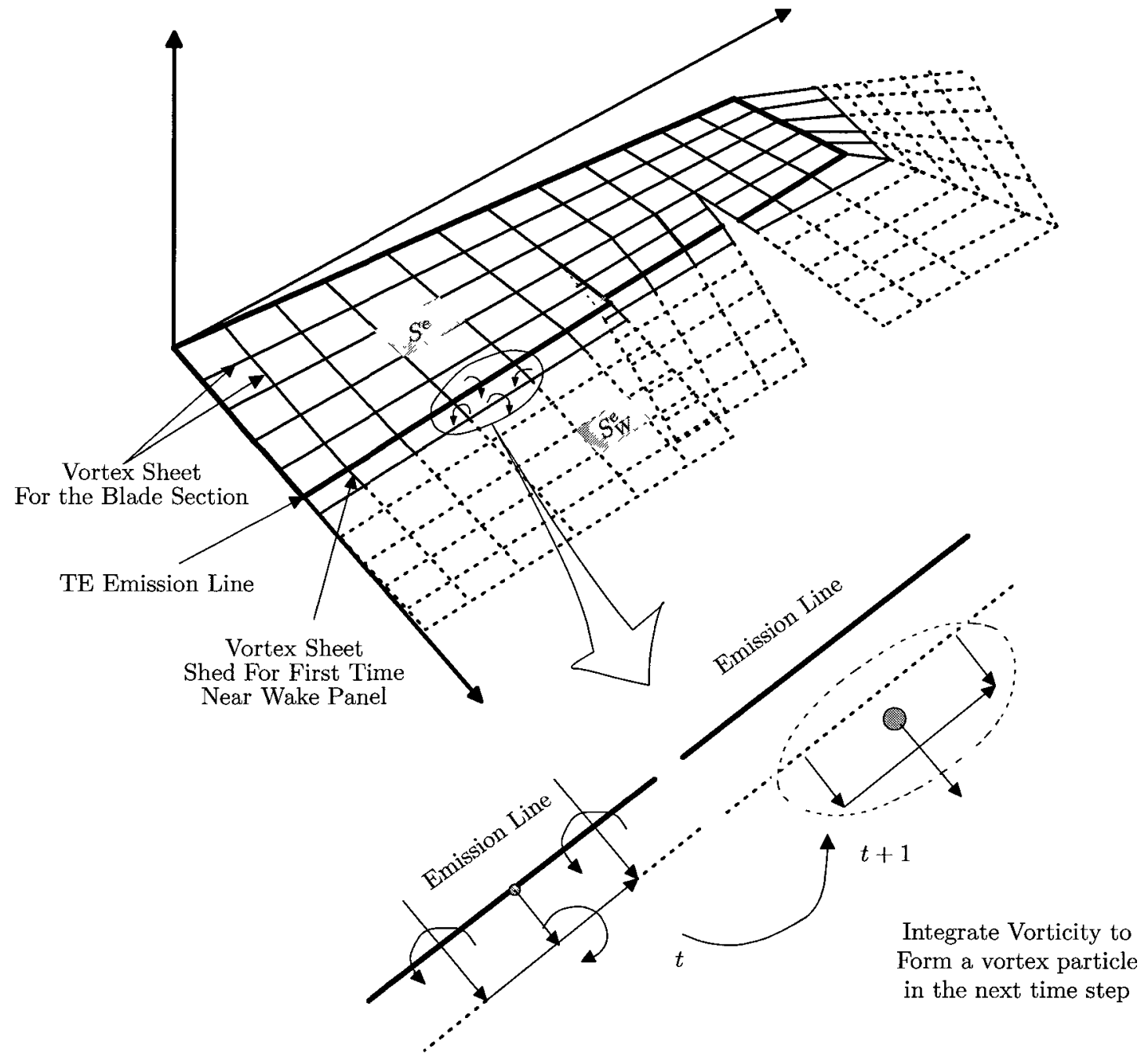

Figure 2.2: Representation of the blade and its wake with vortex sheets and formation of the vortex particle.

For illustrative purposes, in Fig. 2.3 the wake of one blade and the formation of the vortex particles are presented. For this particular simulation, the TEF of the blade was deflected in a sinusoidal manner. The wake was emitted from the trailing edge of a blade and from the sides of the TEF without any separation. For better visualization, the condition of ascending-flight with high collective pitch setting was given to the blade in Fig. 2.3(a) and no aeroelastic coupling was introduced. In addition, the simulations were performed with a small time step. 


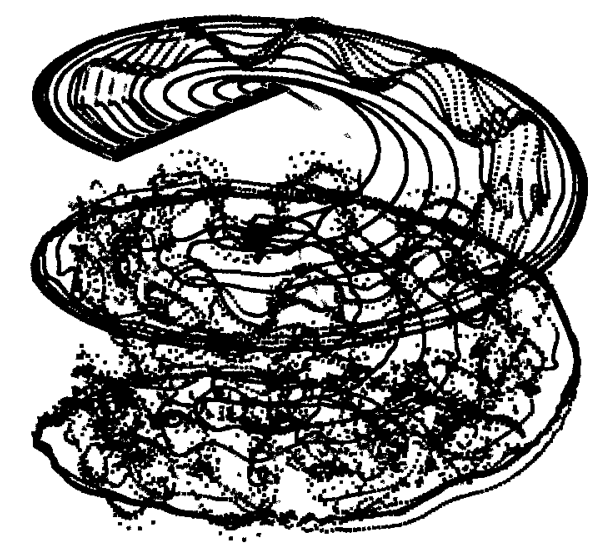

(a) Ascending-flight

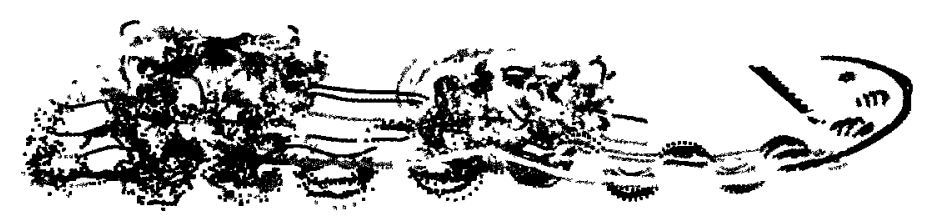

(b) Forward-flight

Figure 2.3: Illustrative example of wake with sinusoidal TEF deflection.

The outputs of the GENUVP are the aerodynamic loads and moments calculated with respect to blade's local coordinate system. When the aerodynamic loads are applied on the nodes, the blade undergoes both rigid-body displacements and elastic deformations. These structural deformations are calculated using the structural component of the aeroelastic code, called GAST. In GAST, flexible structures are represented by a beam model with second-order accuracy. For the solution, the equation of the beam model is discretized using Finite Element (FE) method. The dynamic and structural coupling of the flexible components are performed in the context of a multi-body analysis, in which the flexible components are allowed to undergo rigid-body displacements and elastic deformations.

In Fig. 2.4, the deformed and undeformed frame definitions are shown. The global frame is a rotating reference frame that follows the beam around the rotor azimuth. The undeformed frame accounts for the initial curvature and pre-twist of the beam and the deformed frame includes both rigid and elastic beam deformations. The beam undergoes out-of-plane (flapping) and in-plane (lead-lag) bending with respect to $x \prime$ and $z \prime$ axis, respectively. In 
addition, it experiences extension and torsional motion in the $y \prime$ direction.

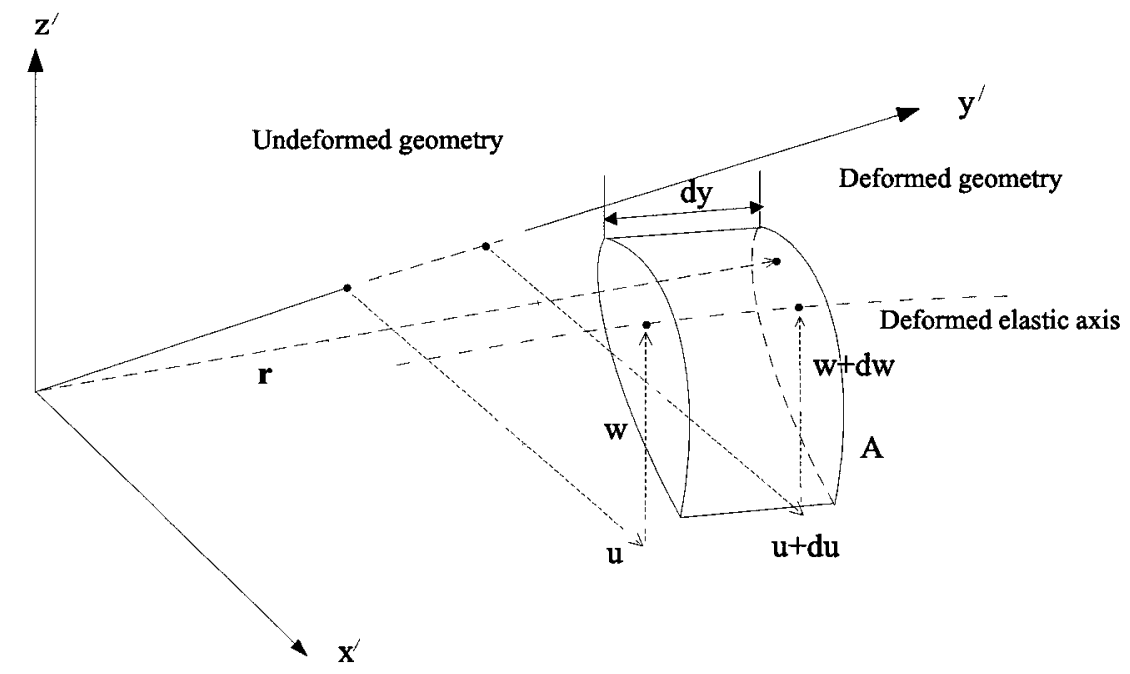

Figure 2.4: A schematic diagram of the coordinate frames used by the structural component.

For the FE formulation, the blade is divided into $N$ number of elements along the span direction. Each element contains 5 nodes, 3 of which located within the element. Figure 2.5 illustrates the element's nodal distribution and its assigned degrees-of-freedom (d.o.f.). The bending d.o.f. is approximated by $3^{\text {rd }}$ order polynomials, and the extension and the torsion by $2^{\text {nd }}$ and $3^{\text {rd }}$ order polynomials, respectively.

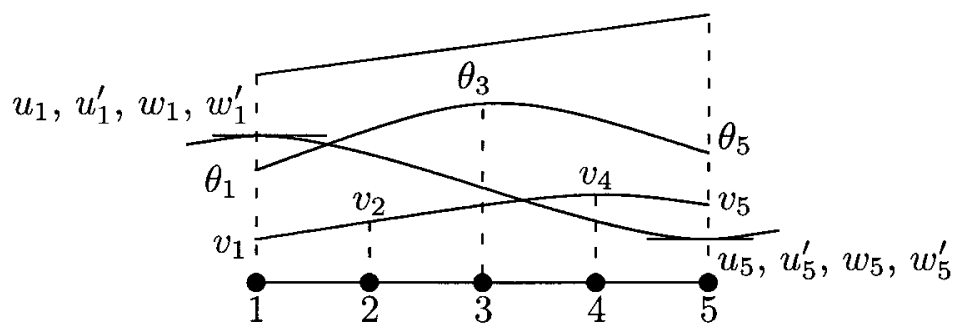

Figure 2.5: Definition of the finite element.

The TEF is modeled as a separate Euler-Bernoulli beam having the elastic axis passing through its hinge and it is connected to the blade by imposing the following boundary conditions. The inner board of the TEF is connected to the blade with a rigid link con- 
nection, which imposes the deformations $u, v, w, \theta$ of the TEF being equal to the levered deformations of blade section at which the TEF is connected. However, the slopes are not constrained. The same boundary conditions were imposed when connecting the outer board of the TEF to the blade, with one exception; the torsional deformation of the TEF, $\theta$ was not constrained.

The rigid-body displacements and elastic deformations of the blade at each node are calculated and the aerodynamic mesh is modified according to the new position of the blade. In addition, the velocity induced by the blade's motion is transferred to the aerodynamic code, GENUVP. Both the aerodynamic loads and the structural deformations are obtained in an iterative aeroelastic solution, i.e. within each time step the aeroelastic coupling is satisfied by means of an iterative process. The result of this aeroelastic analysis produces the aerodynamic loads acting on the blade, blade deformations and the hub loads at any instant of time by transferring the loads from the rotating to the non-rotating frame. Figure 2.6 shows the coordinate system of the rotor with respect to which the blade loads and hub loads are calculated. The blade coordinate system shown in the Fig. 2.6, $(x \prime-y \prime-z \prime)$ corresponds to the blade root axis, which is located aft of the flap, lead-lag and feathering hinges. The rotating frame blade coordinate system, $(x-y-z)$ is located before the flap, lead-lag and feathering hinges. 


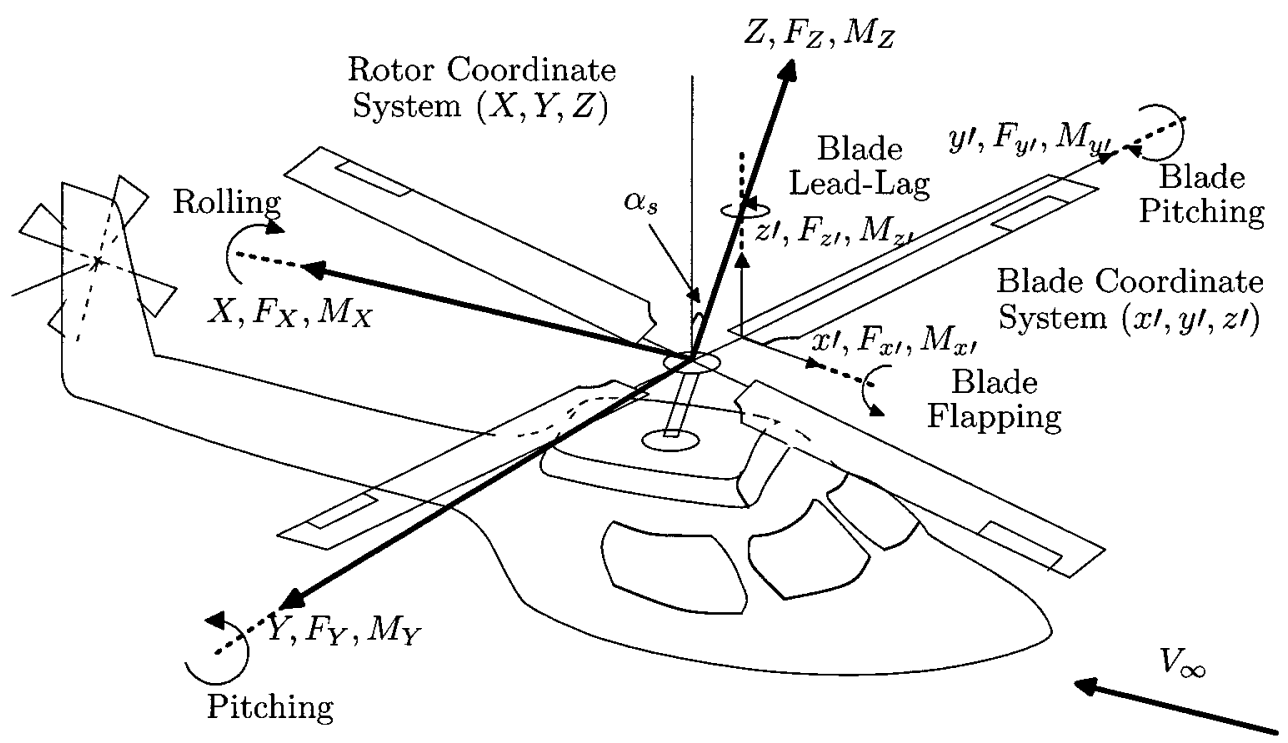

Figure 2.6: Definition of rotor and blade axis of reference in the GENUVP/GAST aeroelastic code.

\subsection{Simulations for Baseline Case}

\subsubsection{SHARCS Blade}

The SHARCS blade has a linear pre-twist distribution with a total twist of 6.75 degrees, and a zero twist distribution between $65 \%$ and $90 \%$ of rotor radius. The operational rotational speed is $\Omega=162.8 \mathrm{rad} / \mathrm{s}(1,555 \mathrm{rpm})$ [16]. A summary of the SHARCS blade properties is given in Table 2.1 . 


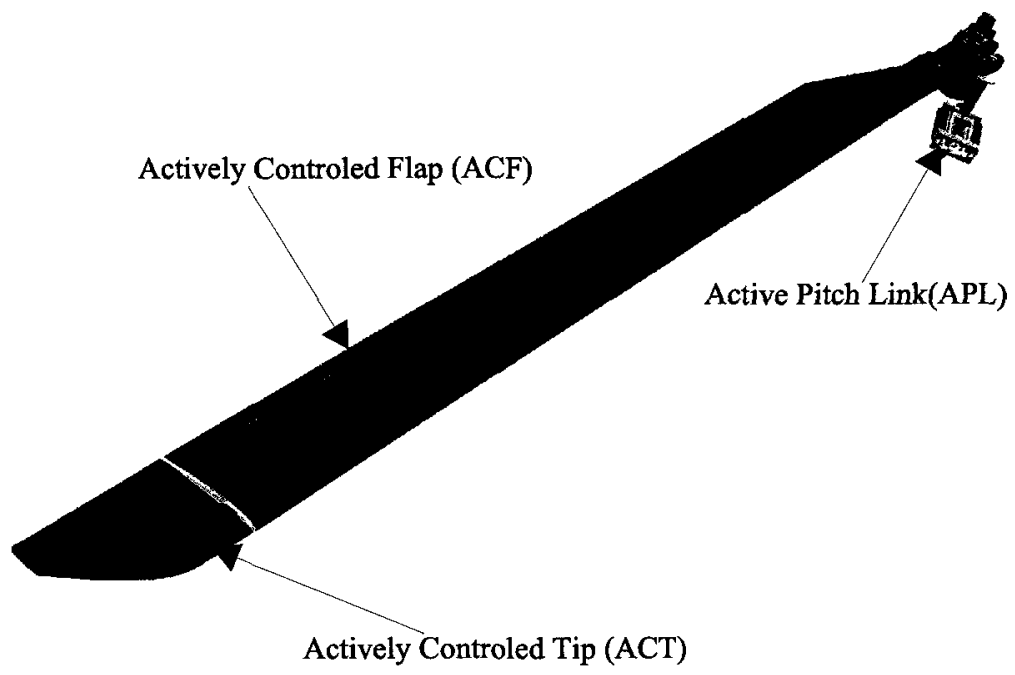

Figure 2.7: The SHARCS blade with three individual feedback systems [16](repeated).

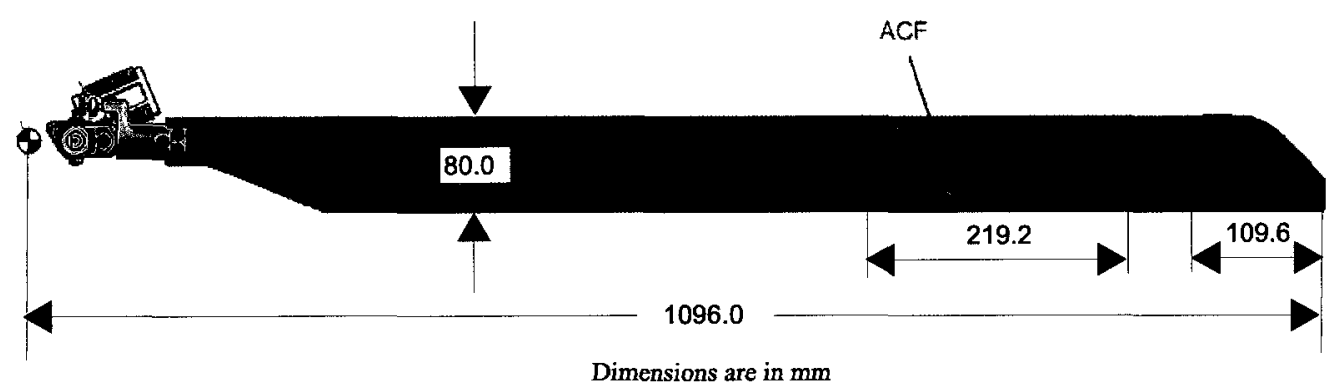

Figure 2.8: Geometric properties of SHARCS blade. 
Table 2.1: Summary of geometric properties of SHARCS blade.

\begin{tabular}{ll}
\hline Rotor Radius & $1096 \mathrm{~mm}$ \\
Rotor Speed $(\Omega)$ & $162.8 \mathrm{rad} / \mathrm{s}$ \\
Rotor Tip Speed & $0.52 \mathrm{Mach}$ \\
Airfoil Section & NACA0015 \\
Chord length & $80 \mathrm{~mm}$ \\
TEF Span & $219.2 \mathrm{~mm}(20 \% \mathrm{span})$ \\
TEF Chord Length & $12 \mathrm{~mm}(15 \%$ chord $)$ \\
\hline
\end{tabular}

The modal frequencies of the SHARCS blade that is equipped with a TEF at the operational speed of $\Omega=162.8 \mathrm{rad} / \mathrm{s}$ are given in Table 2.2. The non-dimensional mode shapes at these frequencies are given in Fig. 2.9. At $\omega=0.24 r e v$, the coupled motion is dominated by lead-lag and at $\omega=1.03 / r e v$ and $\omega=2.96 /$ rev it is dominated by flapping. At frequency $5.23 / \mathrm{rev}$, coupled elastic flapping and torsional modes have the highest contributions. The first elastic lead-lag mode occurs at a frequency $5.26 / \mathrm{rev}$, which is very close to the coupled flapping and torsional modes.

Table 2.2: Eigenfrequencies at a rotational speed, $\Omega=162.8 \mathrm{rad} / \mathrm{s}$.

\begin{tabular}{lc} 
Mode Number & Frequency $(/$ rev $)$ \\
Rigid Lead-Lag & 0.24 \\
Rigid Flapwise Beam Bending & 1.03 \\
Elastic Flapwise Beam Bending & 2.96 \\
Coupled Elastic Flapwise Beam Bending -Elastic Torsion & 5.23 \\
Elastic Chordwise Bending & 5.26 \\
Elastic Torsion & 6.48 \\
Coupled Elastic Flapwise Beam Bending -Elastic Torsion & 9.11 \\
Coupled Elastic Flapwise Beam Bending -Elastic Torsion & 13.69 \\
\hline
\end{tabular}




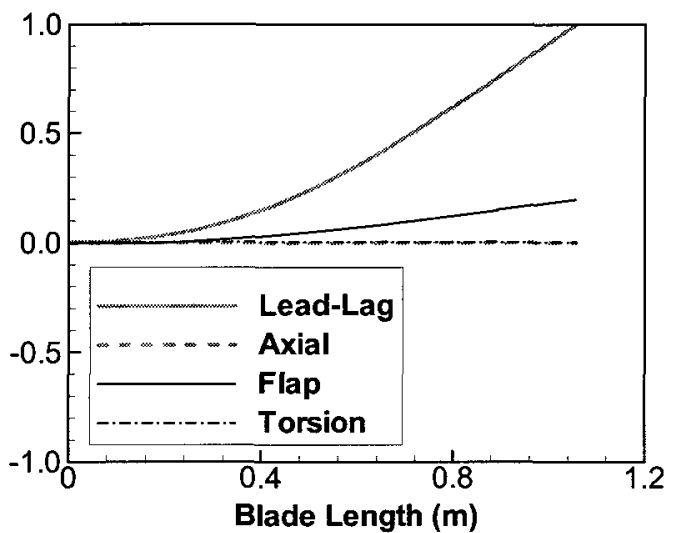

(a) $\omega=0.24 / \mathrm{rev}$

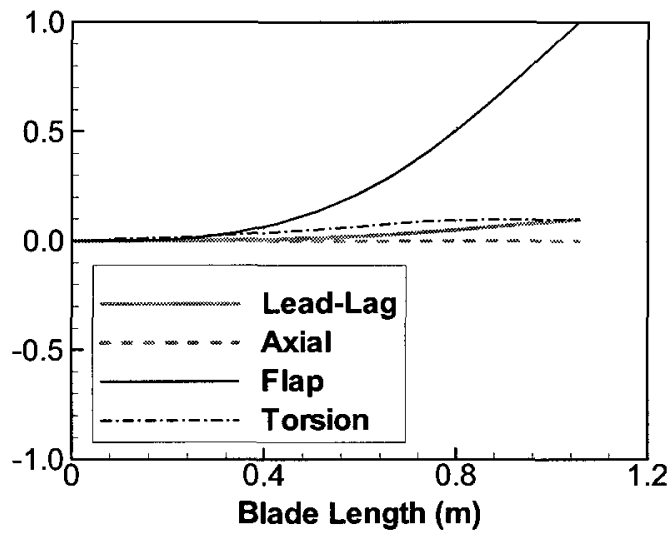

(c) $\omega=2.96 /$ rev

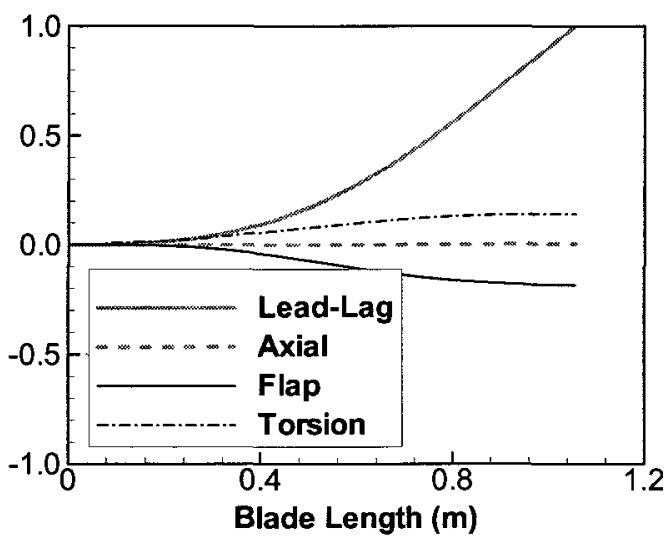

(e) $\omega=5.26 / r e v$

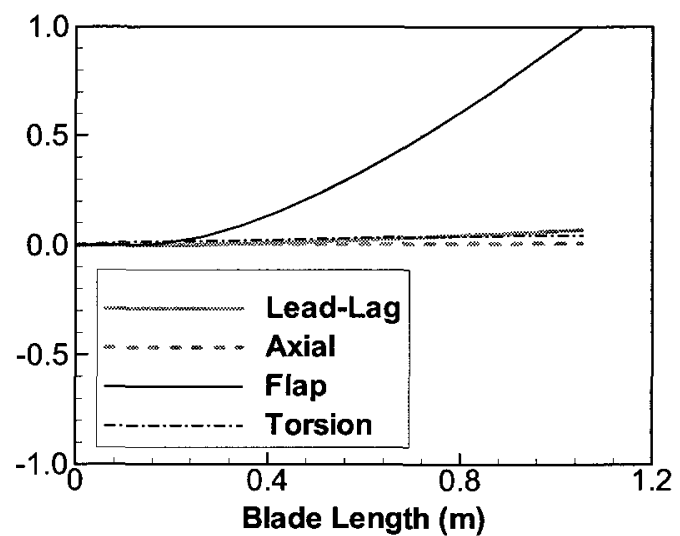

(b) $\omega=1.03 / r e v$

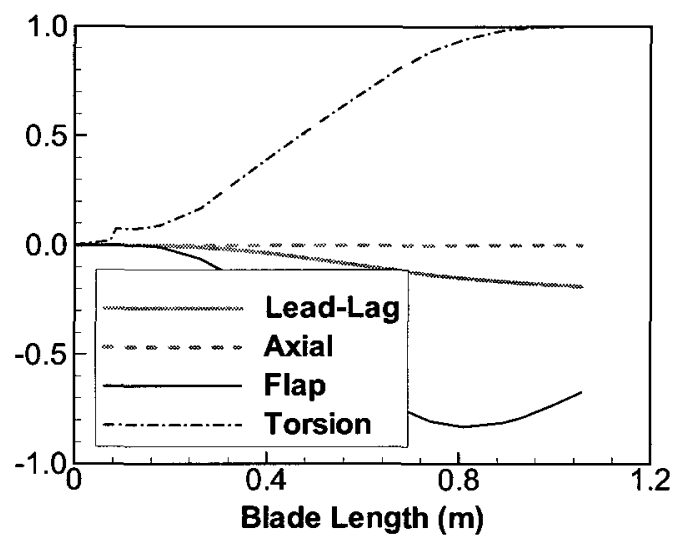

(d) $\omega=5.23 /$ rev

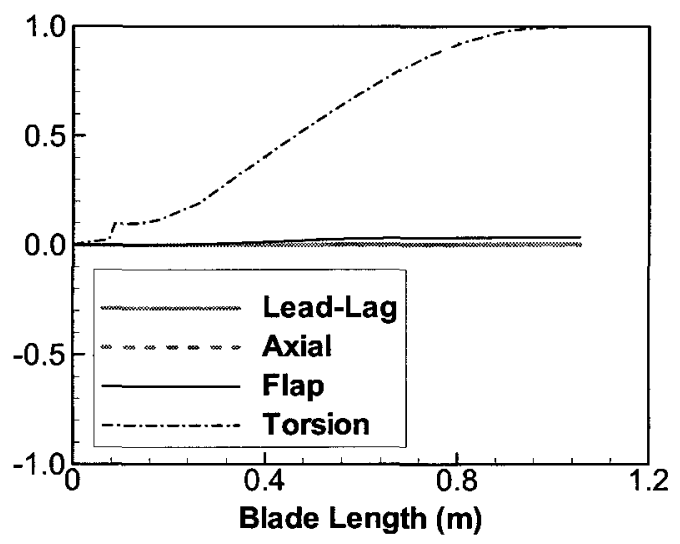

(f) $\omega=6.48 / \mathrm{rev}$

Figure 2.9: Normalized mode shapes at the eigenfrequencies. 


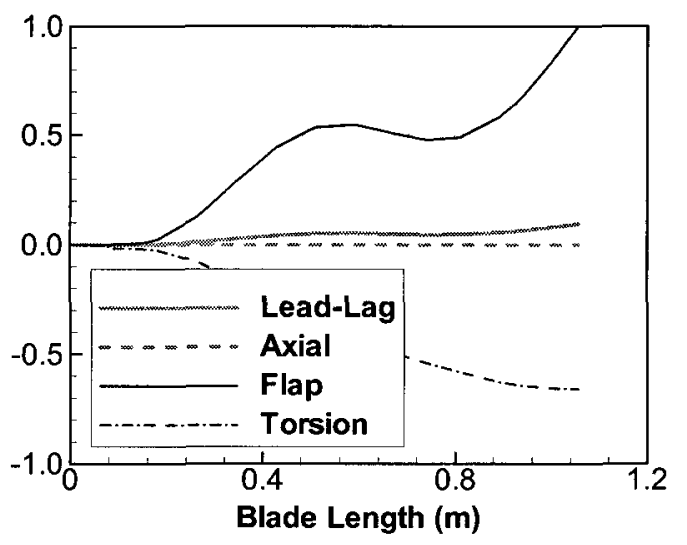

(g) $\omega=9.11 / r e v$

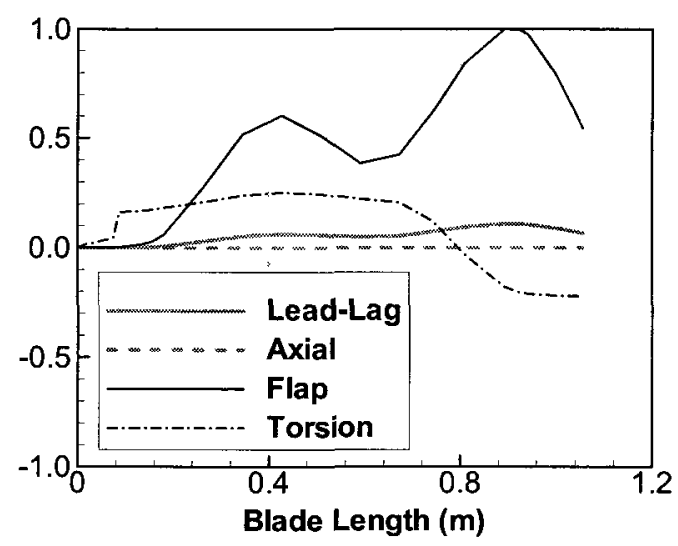

(h) $\omega=13.69 / \mathrm{rev}$

Figure 2.9: Normalized mode shapes at the eigenfrequencies (Cnt).

\subsubsection{Baseline Flight Condition}

The SHARCS blade operates at the baseline forward-flight regime with a rotational speed of $\Omega=162.8 \mathrm{rad} / \mathrm{s}$ and an advance ratio of $\mu=0.28$. The wind tunnel trim condition was satisfied by adjusting the swash plate settings. Wind tunnel trimming requires minimization of the oscillatory rigid flapping amplitude of blade while matching the defined trust coefficient. It does not take into account the loads from fuselage and tail rotor, and it does not require the equilibrium of the longitudinal, lateral and vertical force equations [1]. The trust coefficient, $C_{T}$ was selected to yield $C_{T} / \sigma=0.075$. The forward shaft tilt angle of was taken as $\alpha_{s}=3^{\circ}$. These selected parameters are typical for forward flight regimes. The collective, $\theta_{0}$ and cyclic, $\theta_{1 s}, \theta_{1 c}$ swash plate setting that satisfy the wind tunnel trim condition were obtained from trimming simulations, and produced the values of $\theta=5.42^{\circ}, \theta_{1 s}=-3.72^{\circ}, \theta_{1 c}=1.44^{\circ}$, respectively.

At these swashplate settings, the GENUVP/GAST simulations reached the steady-state values in ten revolutions for the flapping motion, trust, $F_{Z}$ rolling moment, $M_{X}$ and pitching moment, $M_{Y}$ of the helicopter rotor system. However, since the lead-lag motion of the blade has very low damped (no drag correction was applied), it did not reach a perfect steadystate condition. In addition, there is considerable difference in the oscillatory amplitude of the lead-lag motion of each blade. The effect of this difference on the identification of TEF 
deflection on the helicopter's aeroelastic response is analyzed and discussed in Section 4.3. The blades flap about a mean angle of 1.46 degree in the upward direction and lag about a mean angle of 0.5 degree with considerably different oscillatory amplitudes. The baseline hub loads over thirty revolutions and their frequency responses obtained after ten revolutions are presented in Fig. 2.10. 

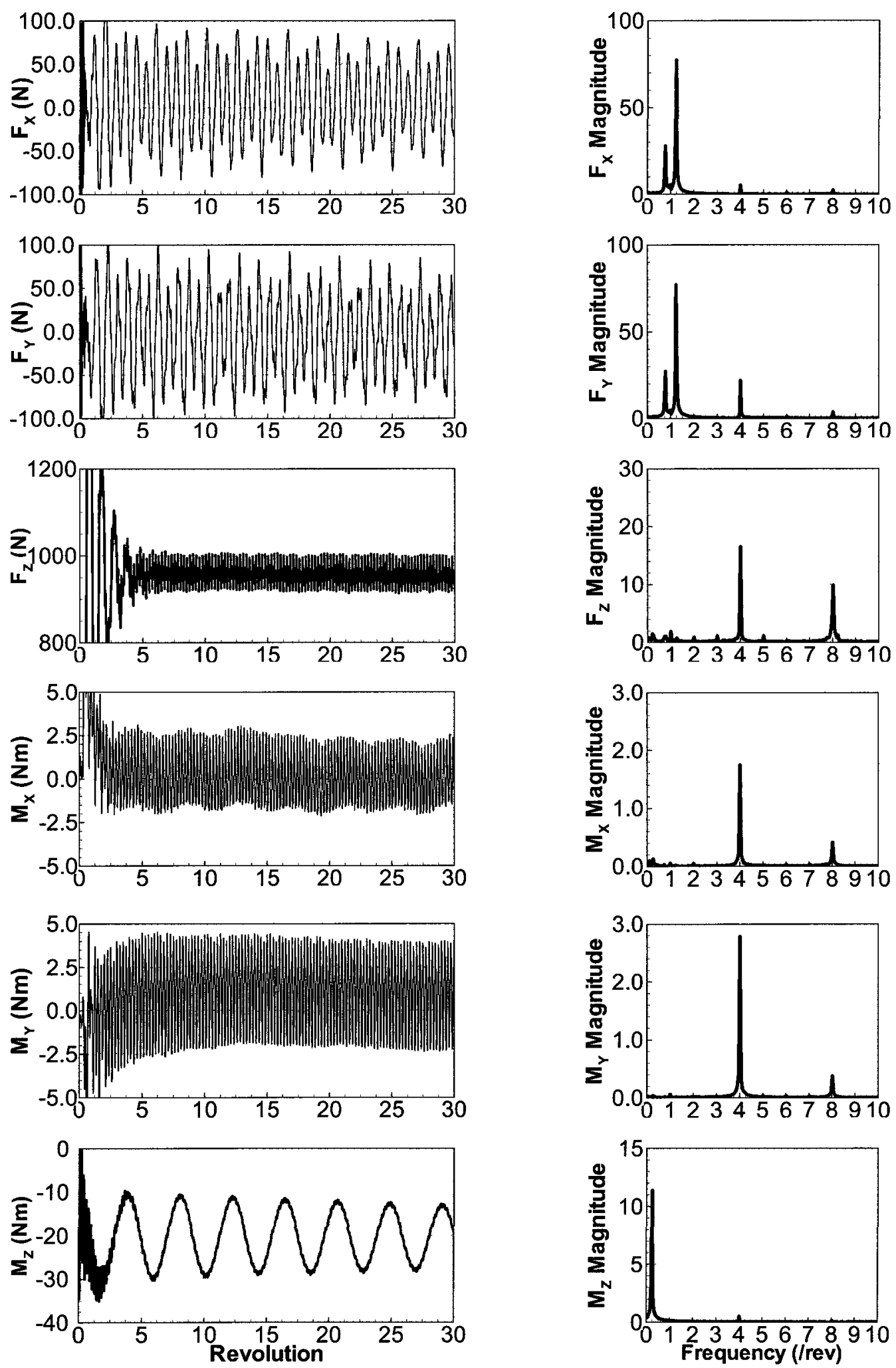

Figure 2.10: Convergence of the baseline case: hub loads, $F_{X}, F_{Y}, F_{Z}, M_{X}, M_{Y}, M_{Z}$. 
The mean trust value over one revolution satisfies the trim condition of the trust coefficient. Also, both rolling moment and pitching moments have approximately zero mean values over one revolution. As seen from the frequency responses of the hub loads, they oscillate about the mean value with harmonics of $N_{b} / r e v$ frequency, where $N_{b}$ is the number of blades. Since only the main rotor was analyzed, the trim value of the yawing moment $M_{Z}$ could not be adjusted to zero.

The rotating frame blade loads, $F_{z}$ and $M_{y}$ that are primarily used for the identification and the control purposes and the corresponding hub loads, $F_{Z}, M_{X}$ and $M_{Y}$ are presented in Fig. 2.11 for two full revolutions after the steady-state baseline flight condition is reached. 

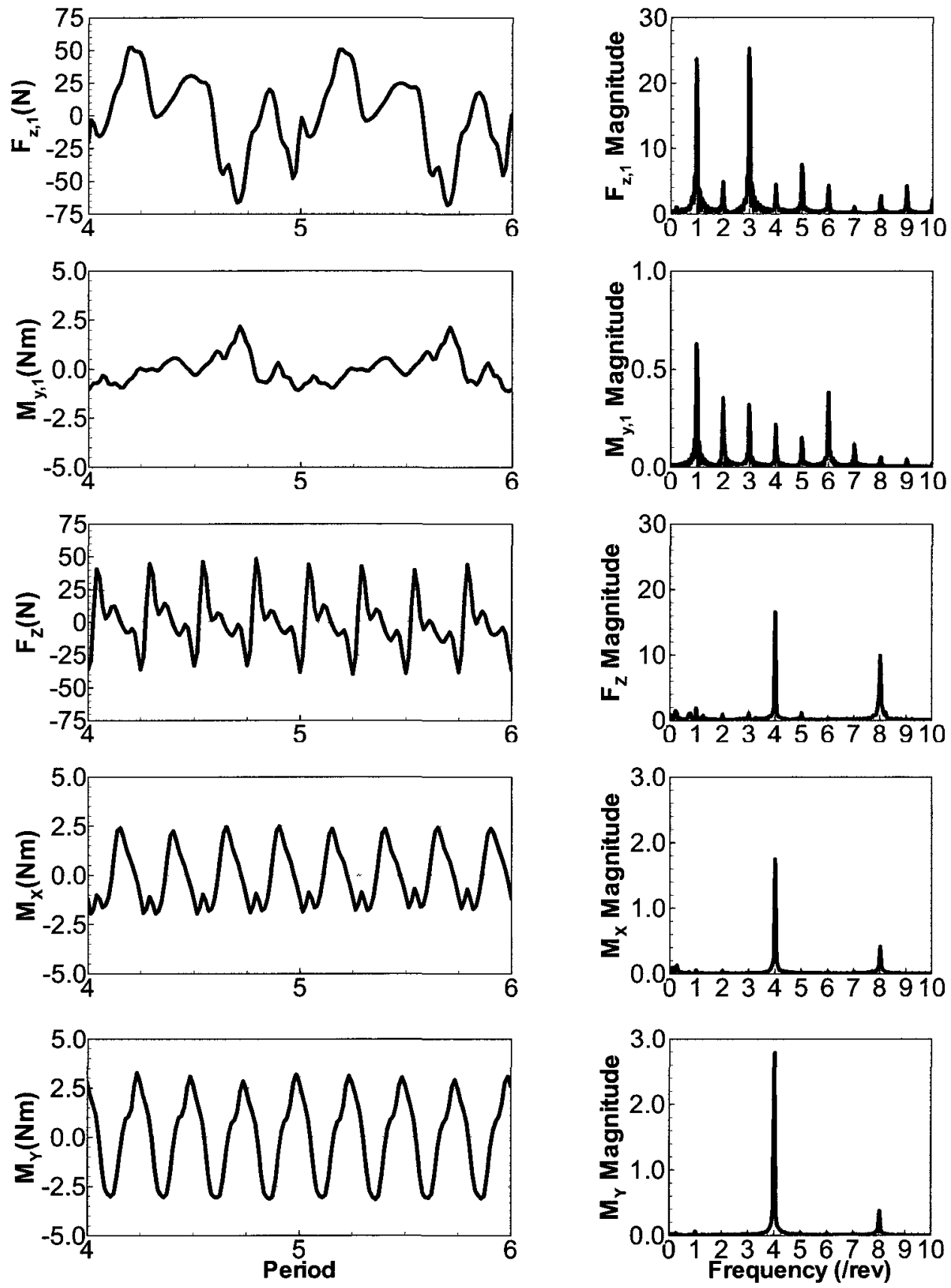

Figure 2.11: Rotating blade loads and hub loads at baseline case.

Compared to Fig. 2.10, where the total frequency response of hub loads in the nonrotating frame is given, the transformation from the rotating frame to the non-rotating frame and the summation of the transformed rotating frame forces produces, as expected, 
the known "filtering effect" for identical rotor blades (loads multiple of the rotating frequency are passed to the non-rotating frame).

\subsection{Assumptions for Baseline Flight Case and Trailing Edge Flap Modeling}

The basic assumptions for the baseline flight conditions of the SHARCS blade are summarized below:

1. The swash plate settings for the baseline case were obtained to satisfy the wind tunnel trim condition for a given advanced ratio and shaft angle. The baseline case for the advance ratio $\mu=0.28$ with forward shaft angle of $\alpha_{s}=3^{\circ}$ and swash plate collective and cyclic settings of $\theta=5.42^{\circ}, \theta_{1 s}=-3.72^{\circ}$, and $\theta_{1 c}=1.44^{\circ}$ approximately reached the steady-state condition ten revolutions after the code started. These settings were kept constant in the identification of the TEF effect on the blade and hub loads.

2. The blades are identical.

3. The elasticity of the shaft and the swash plate is not modeled.

4. No drag correction is performed.

5. For the aerodynamic calculations, the flow was assumed to be attached, and the vortices were emitted from both the trailing edge of the blade and the gap between the blade and the TEF.

6. Rigid TEF deflections were imposed. The dynamics of the TEF and actuation mechanism for TEF were not included in this study.

\subsection{Conclusion}

In this chapter, the computational tool, the GENUVP/GAST time-marching aeroelastic code was introduced and the SHARCS blade properties were presented. The baseline forward flight regime characteristics at the trimmed state were summarized. The baseline 
flight regime shows that the hub loads oscillate around the trimmed state with an expected $N_{b} / r e v$ frequency. The assumptions in the modeling of the aerodynamic and the structural dynamics of the blades and the TEFs were listed as well. 


\section{Chapter 3}

\section{Background of the Applied Theory: System Identification and Controller Design for Time Periodic Systems}

\subsection{Introduction}

In this chapter the summary of the applied theory on the identification of discrete-time LTP systems from ensemble input/output data and the stability conditions for the LTP systems are presented. First, the formal definition of the system is given, then the identification procedure is introduced. The last part of this chapter is dedicated to the formulation of $H_{\infty}$ and $H_{2}$ controller synthesis problems that result in time-periodic controllers.

\subsection{Modeling}

Modeling of a complex physical system can be realized in three distinct ways, namely: fundamental modeling, grey-box modeling, and black-box modeling. Fundamental models can be obtained if a perfect knowledge of the system behavior exists, allowing the application of the fundamental principles of the physics, such as conservation of mass, moment, and energy. Grey-box modeling combines the knowledge on the fundamental principles with observations from the input/output pairs to identify the unknown physical parameters. In black-box modeling, commonly referred as system identification, only the input/output relationships are used in the system description and there is no available direct physical 
interpretation for the system behavior. Using one of these methods, a mathematical model, which provides a map between the input and output spaces, can be obtained.

Since, the helicopter's aeroelastic response involves the solution of the flow field around the helicopter blade, which is coupled with both the rigid body and elastic deformation of the blades, the overall system cannot be described from simple fundamental principles. Hence, system identification was applied to model the helicopter's aeroelastic response caused by the deflection of the trailing edge flaps (TEF). In the system identification, the model was chosen to be the LTP because of the observed time-periodic behavior of helicopter aeroelastic response in forward flight regime. In addition, since the model was intended to be used to design a controller whose effectiveness can be tested in aeroelastic computer simulations, the LTP model was formulated in discrete time. Moreover, statespace modeling was chosen because the helicopter's aeroelastic model will be used to design controllers using $H_{\infty}$ and $H_{2}$ design framework, which has been developed using state-space models.

\subsubsection{System Definition}

Operator mapping from the input to the output space of a system shows different properties depending on the system characteristics such as linear or non-linear, time-invariant or timevarying. In this mapping, the input space is composed of controlled and uncontrolled input variables such as disturbances, noise and reference signal. It is represented by a vector, $u \in \mathbb{R}^{m}$ with $m$ being the number of inputs. The output space, composed of measured and controlled output variables such as performance measures (i.e. error signal, actuator limitations), is represented by a vector $y \in \mathbb{R}^{p}$ with $p$ being the number of outputs. The mapping used in this study is considered to be causal, linear, and periodic. The reason being as follows:

Causal because the system's output, $y(t)$ at any time instant, $t$ is only affected by the inputs at the present time, $t$ and the inputs applied in the past. All the physically realizable systems are causal.

Linear because the total output caused by the linear combination of $N$ inputs is equal 
to the linear combination of scaled outputs caused by the individual inputs.

$$
\begin{aligned}
& u(t)=\sum_{\imath=1}^{N} \alpha_{\imath} u_{\imath}(t) \\
& y(t)=\sum_{\imath=1}^{N} \alpha_{\imath} y_{\imath}(t), \quad \forall \alpha_{\imath} \in \mathbb{R}
\end{aligned}
$$

Periodic because there exists a time $T$ such that, when an input $u(t)$ is applied to the system at time $t_{o}$ and the system responds to this input as $y(t)$, if the same input is applied at $t_{o}+T$ and the system response at $t+T$ is equal to $y(t)$, i.e.;

$$
u\left(t_{o}\right)=u_{d}\left(t_{o}+T\right), \quad y(t)=y_{d}(t+T)
$$

where, $u_{d}$ and $y_{d}$ are used to represent one period delayed input and output.

The smallest $T$ satisfying the above equality is called the period of the system and the system is then called $T$-periodic system. If a system obeys the three properties stated above, then it is called Linear Time Periodic system. If a zero-order-hold device is used in a LTP system, assuming that the system is constant along the sampling period, $T_{s}$, the resultant system becomes discrete-time LTP with period $N=\frac{T}{T_{s}}$.

\subsection{Overview on Linear Time Periodic Systems}

For causal discrete-time LTP systems, the input/output relationship (the output at time $t_{k}, y\left(t_{k}\right)$ as a result of all past inputs) can be written using periodic Markov parameters, $M_{\imath}\left(t_{k}\right), M_{\imath}\left(t_{k}\right)=M_{\imath}\left(t_{k}+N\right)$, where $t_{k} \in \mathbb{Z}$ refers to a discrete-time index and $N \in \mathbb{Z}$ is used to define the discrete-time period.

$$
\begin{aligned}
y\left(t_{k}\right) & =M_{o}\left(t_{k}\right) u\left(t_{k}\right)+M_{1}\left(t_{k}\right) u\left(t_{k}-1\right)+M_{2}\left(t_{k}\right) u\left(t_{k}-2\right)+\cdots \\
& =\sum_{\jmath=0}^{\infty} M_{\jmath}\left(t_{k}\right) u\left(t_{k}-j\right)
\end{aligned}
$$

For $t_{k}=p N+k$, the output $y\left(t_{k}\right)$ is written in terms of the output of a LTI system, $\hat{y}_{\imath, k}(p)$ as given in Eq. 3.5b. In Eq. 3.5b, $M_{\imath, k}, M_{N+\imath, k}, \cdots, M_{\jmath N+\imath, k}$ represents the Markov 
parameters of the time invariant system. The tag time index for the output and input variables are $k$ and $k-i$ respectively, and $p$ shows the number of periods completed [120].

$$
y_{p N+k} \quad=\sum_{\imath=0}^{N-1} \hat{y}_{\imath, k}(p)
$$

where

$$
\begin{aligned}
& \hat{y}_{\imath, k}(p) \quad=\sum_{\jmath=0}^{\infty} M_{\jmath N+\imath, k} \hat{u}_{\imath, k}(p-j) \\
& \hat{u}_{\imath, k}(p-j)=u_{(p-\jmath) N+k-\imath}
\end{aligned}
$$

This global mapping between the input and output when decomposed into a sequence of local operations is shown in Figure 3.1, where new variables are needed to describe these operations. These intermediate quantities pass the history of the system for future references, and so they are called "states" of the system [121]. The states of the system do not necessarily have a physical meaning and they are not unique. Also, as seen in Figure 3.1, for time-varying systems the number of states, $n_{k}$ does not need to be constant.

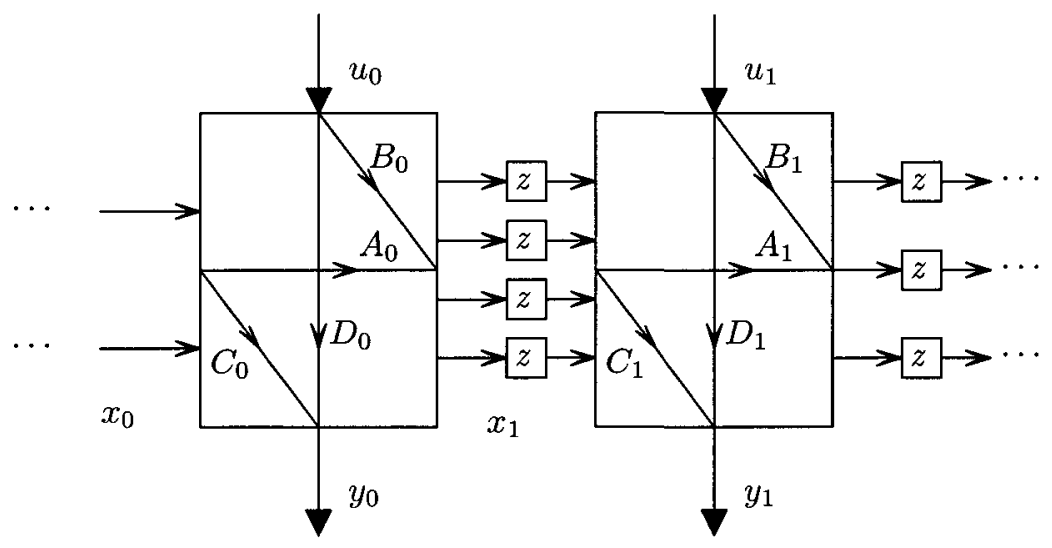

Figure 3.1: State-space modeling of time-varying systems [121].

In Fig. 3.1, $z$ shows the time shift operator and $A_{k}, B_{k}, C_{k}, D_{k}$ are the discrete-time time-periodic state-space matrices with which the system's input/output relationship is 
written using the states as defined in Eq. 3.6.

$$
\left[\begin{array}{c}
x_{k+1} \\
y_{k}
\end{array}\right]=\left[\begin{array}{cc}
A_{k} & B_{k} \\
C_{k} & D_{k}
\end{array}\right]\left[\begin{array}{l}
x_{k} \\
u_{k}
\end{array}\right]
$$

where

$$
\begin{array}{ll}
A_{k+N}=A_{k} \in \mathbb{R}^{n_{k} \times n_{k}}, & B_{k+N}=B_{k} \in \mathbb{R}^{n_{k} \times m_{k}} \\
C_{k+N}=C_{k} \in \mathbb{R}^{p_{k} \times n_{k}}, & D_{k+N}=D_{k} \in \mathbb{R}^{p_{k} \times m_{k}}
\end{array}
$$

The Markov parametes can be written in terms of the discrete-time state-space matrices as shown in Eq. 3.7 [122].

$$
\begin{aligned}
& M_{o, k}=D_{k} \\
& M_{1, k}=C_{k} B_{k-1} \\
& M_{2, k}=C_{k} A_{k-1} B_{k-2} \\
& M_{3, k}=C_{k} A_{k-1} A_{k-2} B_{k-3} \\
& \quad \vdots \\
& M_{p T+\imath, k}=C_{k} \Psi_{k}^{p} \Phi_{k, k-\imath+1} B_{k-\imath}
\end{aligned}
$$

where $\Phi_{k, \tau}$ with $k \geq \tau$ is the state-transition matrix, and $\Psi_{\tau}$ is called monodromy matrix, whose eigenvalues do not depend on $\tau$. The definitions of $\Phi_{k, \tau}$ and $\Psi_{\tau}$ are given in Eq. 3.8a and Eq. 3.8b, respectively.

$$
\begin{aligned}
& \Phi_{k, \tau}=\left\{\begin{array}{lll}
A_{k-1} A_{k-2} \cdots A_{\tau} & \text { if } \quad k \geq \tau \\
I & \text { if } \quad k=\tau
\end{array}\right\} \\
& \Psi_{\tau}=\Phi_{N+\tau, \tau}
\end{aligned}
$$

\subsection{State-Space Realization from Ensemble Input/Output Data Pairs}

The state-space model of the system, $A_{k}, B_{k}, C_{k}, D_{k}$, can be obtained using the input/output data of the system utilizing a subspace identification method. Subspace identification does not require precise, a prıor knowledge of the model parameters. Therefore, a more efficient 
system identification using shorter experimental and/or computational input/output data sets is possible. Also, the subspace identification method (SIM) is capable of identifying the system model using noise corrupted input/output data sets acquired from different experiment runs $[69,123]$. In this work, the SIM was carried out using the Multivariable OutputError State-sPace (MOESP) algorithm for time-varying systems. The MOESP algorithm is based on recovering the column space of the extended observability matrix and uses the shift-invariant structure of this matrix to estimate the matrices $A_{k}$ and $C_{k}[69,121,123]$.

Although, the precise information of the model structure is not needed, an approximate order of the system is necessary to define the total number of experiments to be performed, $n$ and the duration of each experiment, $s[69,123]$. The ensemble input/output sequences can be either obtained by performing different experiments or just one experiment with a length $M_{\text {tot }}$. By using ensemble input/output data, the input/output relation was analyzed at times $N+k, 2 N+k, \cdots,(n-1) N+k$, at which the system has identical characteristics (see Eq. 3.6). The input/output sequence is defined in Eq. 3.9.

$\begin{array}{llll}y_{k} & y_{k+1} & \ldots & y_{k+s-1} \\ y_{N+k} & y_{N+k+1} & \ldots & \cdot \\ \vdots & & & \vdots \\ y_{(n-2) N+k} & & \ldots & \cdot \\ y_{(n-1) N+k} & & \ldots & y_{M_{t o t}}\end{array}$

where the minimum number of experiments, $n_{\min }$ and the total experiment length, $M_{t o t}$ are defined as

$$
n_{\min }=\max _{k \in[1, N]}\left(M_{k}^{s}+n_{k}\right) \quad \text { and } \quad M_{\text {tot }_{\min }}=n_{\min } N+s-1
$$

where

$$
M_{k}^{s}=\sum_{\tau=k}^{k+s-1} m_{\tau} \quad \text { and } \quad P_{k}^{s}=\sum_{\tau=k}^{k+s-1} p_{\tau}
$$

The corresponding generalized input and output Hankel matrices, $U_{k, s} \in \mathbb{R}^{n \times M_{k}^{s}}$ and $Y_{k, s} \in \mathbb{R}^{n \times P_{k}^{s}}$, respectively are written for $k \in[1, N]$. Equation 3.12 shows only the output 
Hankel matrix, $Y_{k, s}$ the input Hankel matrix, $U_{k, s}$ is written by replacing the output, $y$ with input, $u$.

$$
Y_{k, s}=\left[\begin{array}{llll}
y_{k} & y_{k+1} & \cdots & y_{k+s-1} \\
y_{N+k} & y_{N+k+1} & \cdots & \cdot \\
\vdots & & & \vdots \\
y_{(n-2) N+k} & & \cdots & \cdot \\
y_{(n-1) N+k} & & \cdots & y_{(n-1) N+k+s-1}
\end{array}\right]
$$

When the system is excited by random inputs, both the input and output Hankel matrices are formed as given in Eq. 3.12 for $k \in[1, N]$. Using input/output Hankel matrices, the compound matrices, $\left[\begin{array}{l:l}U_{k, s} & Y_{k, s}\end{array}\right] k \in[1, N]$, are obtained. They were then factorized using the QR factorization method as shown in Eq. 3.13:

$$
\left[\begin{array}{l:l}
U_{k, s} & Y_{k, s}
\end{array}\right]=\left[\begin{array}{l:l}
Q_{1_{k}} & Q_{2_{k}}
\end{array}\right]\left[\begin{array}{c:c}
R_{11_{k}} & R_{12_{k}} \\
\hdashline 0 & R_{22_{k}}
\end{array}\right]
$$

where the dimension of the partitioned matrix $R$ is $R_{11_{k}} \in \mathbb{R}^{M_{k}^{s} \times M_{k}^{s}}, R_{11_{k}} \in \mathbb{R}^{M_{k}^{s} \times P_{k}^{s}}$, and $R_{22_{k}} \in \mathbb{R}^{n-M_{k}^{s} \times P_{k}^{s}}$. Matrix $Q$ contains the orthonormal basis with the properties $Q_{1_{k}}^{T} Q_{1_{k}}=I_{M_{k}^{s}}$ and $Q_{1_{k}}^{T} Q_{2_{k}}=0$. The row space of, $R_{22, k}$ is equal to the row space of the observability matrix, $\mathcal{O}_{k}$, which is defined in Eq. 3.14 along with the controllability matrix, $\mathcal{C}_{k}$. By using the SVD decomposition of the matrix $R_{22, k}$ (see Eq. 3.15), the extended observability matrix was obtained to find the state-space matrices $A_{k}, C_{k}$ as follows:

$$
\begin{aligned}
& \mathcal{O}_{k}=\left[\begin{array}{c}
C_{k} \\
C_{k+1} A_{k} \\
C_{k+2} A_{k+1} A_{k} \\
\vdots
\end{array}\right] \\
& \mathcal{C}_{k}=\left[\begin{array}{llll}
B_{k-1} & A_{k-1} B_{k-2} & A_{k-1} A_{k-2} B_{k-3} & \cdots
\end{array}\right]
\end{aligned}
$$




$$
R_{22, k}=\left[\begin{array}{c}
U_{k} \\
\hdashline\left(U_{k}^{\perp}\right)
\end{array}\right]\left[\begin{array}{c:c}
\Sigma_{n_{k}} & 0 \\
\hdashline 0 & \Sigma_{0_{k}}
\end{array}\right]\left[\begin{array}{c}
\left(V_{k}\right)^{T} \\
\hdashline\left(V_{k}^{\perp}\right)^{T}
\end{array}\right]
$$

where, $\Sigma_{n_{k}}$ gives the singular values of $R_{22, k}$ and $\Sigma_{n_{k}} \in \mathbb{R}^{n_{k} \times n_{k}}$. The order of the system at time instant $k$ is defined by the number of singular values taken into account. In this work, $r$ is number of singular values of the $R_{22, k}$ matrix that are selected at each time instant $k$ by pre- or post-multiplying the matrix $R_{22, k}$ by the operator $\pi_{r(k)}=\left[\begin{array}{llll}I_{r} & 0 & 0 & \cdots\end{array}\right] .\left(\pi_{r(k)}\right.$ selects the $r(k)$ number of rows of the $R_{22, k}$ matrix and its conjugate transpose, $\pi_{c(k)}^{*}$ selects the $c(k)$ number of columns of the $R_{22, k}$ matrix). The reduced-dimension $\hat{R}_{22, k}$ are then formed. From the reduced order observability matrix, $\hat{\mathcal{O}}_{k}$, the state-space matrices $\hat{A}_{k}, \hat{C}_{k}$ are obtained via Eq. 3.16a. The periodicity of the system is imposed by using an appropriate row space of the extended observability matrix $\mathcal{O}_{k}$ at various time instances [69].

$$
\begin{aligned}
& \hat{A}_{k}=\hat{\mathcal{O}}_{k+1} \hat{\mathcal{O}}_{k+1}^{\dagger} \quad \text { and } \quad \hat{C}_{k}=\hat{\mathcal{O}}_{k} \pi_{1}^{*} \\
& \hat{\mathcal{O}}_{k}=\mathcal{O}_{k} \pi_{c(k)}^{*} \quad \text { and its pseudo-inverse } \quad \hat{\mathcal{O}}_{k}^{\dagger}=\hat{\mathcal{O}}_{k}^{*}\left[\hat{\mathcal{O}}_{k} \hat{\mathcal{O}}_{k}^{*}\right]^{-1}
\end{aligned}
$$

For the solution of matrices $B_{k}$ and $D_{k}$, an alternative method was used, which is based on the minimization of the output error of the identified system where $\hat{A}_{k}$ and $\hat{C}_{k}$ are known [84]. The output of the model is re-written by substituting the definition of the Markov parameters (Eq. 3.7) into Eq. 3.5b as follows:

$$
\hat{y}_{p N+k}=\hat{D}_{k} u_{p N+k}+\sum_{\jmath=0}^{p} \sum_{\imath=1}^{k} \hat{C}_{k} \Psi(k)^{\jmath} \hat{\Phi}(k, k-\imath+1) \hat{B}_{k-\imath} u_{\jmath N+k-\imath}
$$

By using the Kronecker's matrix product, denoted as $\otimes$, and the notation of vec(.), to define a vector formed by stacking the columns of a matrix, Eq. 3.17 can be written as [84]:

$$
\hat{y}_{p N+k}=\left[u_{p N+k} \otimes I_{m}\right]^{\prime} \operatorname{vec}\left(\hat{D}_{k}\right)+\left(\sum_{\jmath=0}^{p} \sum_{\imath=1}^{k} u_{\jmath N+k-\imath} \otimes \hat{C}_{k} \hat{\Psi}(k)^{\jmath} \hat{\Phi}(k, k-i+1)\right) \operatorname{vec}\left(\hat{B}_{k-\imath}\right)
$$

Therefore, $\hat{B}_{k}$ and $\hat{D}_{k}$ can be solved using the solution of Eq. 3.19 to minimize the error 
between the actual output of the system and the identified output:

$$
\hat{B}_{k}, \hat{D}_{k}=\arg \min _{\hat{B}_{k}, \hat{D}_{k}}\left[y_{p N+k}-\hat{y}_{p N+k}\right]^{2}
$$

Once the system model is obtained in the state-space form, it needs be validated. For the validation, the response of the model is compared with the response obtained from the full-order system. Full-order system comparison results are obtained either from the measurements done in the experiments or from computer simulations obtained from a comprehensive analysis of the system. If the error is higher than a defined criteria, the order of the system is increased.

\subsection{Stability of LTP Systems}

The stability of a discrete-time LTI system is defined in terms of the eigenvalues of the dynamic matrix, $A$. If the eigenvalues of $A$ are inside the unit circle (spectral radius, $\rho(A)<1$, the LTI system is stable. On the other hand, the stability of a LTP system, described in Eq. 3.6, does not depend on the stability of the individual dynamic matrices, $A_{k}$

obtained at each time instant, $k$. Even if at a specific time instant $k$, one of the eigenvalues of $A_{k}$ lie outside the unit circle, the LTP system can still be stable. As introduced by FloquetLyapunov, the stability of LTP system is based on the eigenvalues of the monodromy matrix, which are called characteristic multipliers, $\lambda\left(\Psi_{k}\right)$ (Eq. 3.20):

$$
\begin{aligned}
& \Psi_{k}=\Phi_{k+N, k}=A_{k+N-1} A_{k+N-2} \cdots A_{k} \\
& \Lambda=\Lambda_{k}=\lambda\left(\Psi_{k}\right)
\end{aligned}
$$

Therefore, the LTP system defined in Eq. 3.6 is stable if the eigenvalues of the monodromy matrix lie inside the unit circle [120].

Another stability condition is provided by the Lyapunov's stability theory. A function, $V_{k}\left(x_{k}\right)$ is called a Lyapunov's candidate function if it satisfies the conditions given by 
Eq. $3.21[52]:$

$$
\begin{aligned}
& V_{k}(0)=0, \forall k \in \mathbb{Z} \\
& V_{k}\left(x_{k}\right)>0, \forall x_{k} \neq 0, \forall k \in \mathbb{Z} \quad \text { (Positive Definite) } \\
& \lim _{|| x_{k} \| \rightarrow \infty} V_{k}\left(x_{k}\right)=\infty
\end{aligned}
$$

The system is said to be asymptotically stable if the Lyapunov function satisfies the inequality given in Eq. 3.22:

$$
V_{k+1}\left(x_{k+1}\right)-V_{k}\left(x_{k}\right)<0, \forall k \in \mathbb{Z} \quad \text { (Negative Definite) }
$$

If $V\left(x_{k}\right)$ is associated with the energy of the system, $V_{k}(0)=0$ states that at the equilibrium

condition $x_{e q}=0$, the system has zero energy, and $V_{k+1}\left(x_{k+1}\right)-V_{k}\left(x_{k}\right)<0$ guarantees that the energy of the system is decreasing with time, i.e. all possible system evolution trajectories converge to zero [52]. The condition on the asymptotic stability is given by the existence of $N$ periodic symmetric positive definite matrices $P_{k} \in S^{n+} \forall k=1,2, \cdots, N$ that satisfy Eq. 3.23:

$$
\begin{aligned}
& V_{k}\left(x_{k}\right)=x_{k}^{\prime} P_{k} x_{k} \quad \text { where } \quad P_{k+N}=P_{k} \\
& A_{k}^{\prime} P_{k+1} A_{k}-P_{k}<0, \quad \forall k \in 1,2, \cdots, N \\
& P_{k+N}=P_{k}
\end{aligned}
$$

The requirement given in Eq. 3.23 is a special form of Linear Matrix Inequality (LMI) condition (Appendix A p.170) $[88,97,124]$.

\subsection{LTP $H_{\infty}$ and $H_{2}$ Control Synthesis}

This section gives the formulation of the control synthesis problem for $H_{\infty}$ and $H_{2}$ controllers. First, the general block diagram representation is presented. Then for each controller design method, the necessary conditions and the solution methods are given.

A general block diagram representation shown in Fig. 3.2 defines the plant and its environment with disturbances, noise, and limitations in the actuation. It should be noted that, in the framework of this study the uncertainties in the plant model were not considered. In this block diagram representation, $P$ is the nominal plant model, i.e. the state-space 


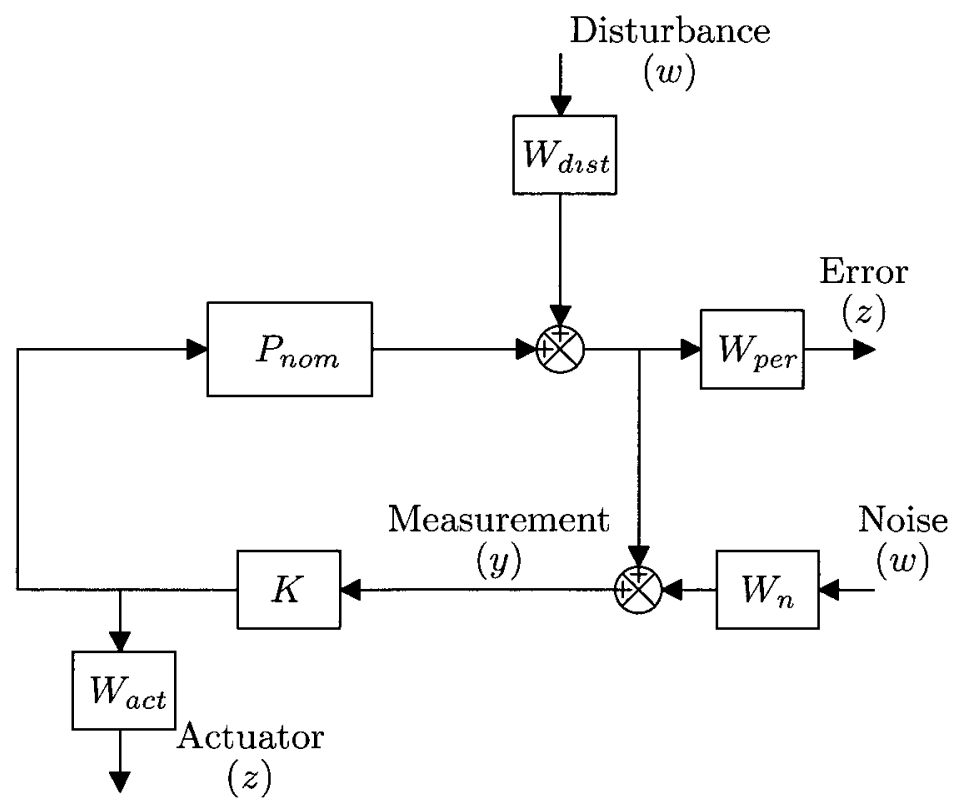

Figure 3.2: General feedback block diagram representation.

model obtained from system identification, the weights $W_{\text {act }}$ and $W_{\text {per }}$ define the actuation limitation and the performance criteria for the output control, respectively. $W_{d i s t}$ and $W_{n}$ show the disturbances in the plant's environment and the error in the measurement, respectively, and $K$ is the controller. Embedding all these weights in matrix $M$, collecting all the disturbances in vector $w_{k}$, and the performance measures in vector $z_{k}$, the general block diagram representation is reduced to the form given in Fig. 3.3, which is called a Linear Fractional Transformation (LFT) [88].

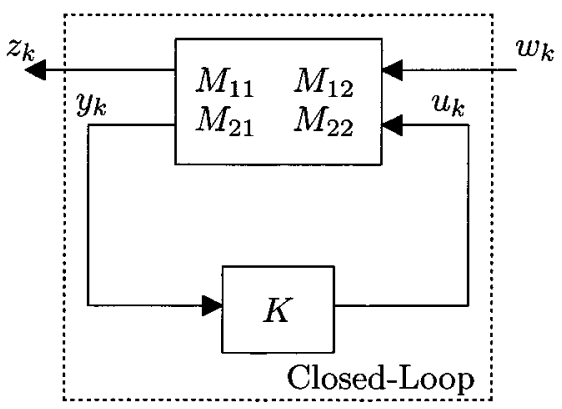

Figure 3.3: Lower linear fractional transformation form. 
The model $M_{k}$ has $n_{M_{k}}=n_{k}+n_{\text {per }}+n_{\text {dist }}+n_{\text {act }}$ number of states, where $n_{k}, n_{\text {per }}, n_{\text {dist }}, n_{\text {act }}$ are the number of states for the nominal plant model $P_{n o m_{k}}$, and weights $W_{\text {per }}, W_{\text {dist }}, W_{\text {act }}$ respectively. The system model has $m_{w_{k}}=m_{\text {dist }}+m_{\text {noise }}$ number of disturbance inputs, $n_{u_{c}}$ number of control inputs, $p_{z_{k}}=p_{p e r}+p_{a c t}$ number of performance outputs, and $p_{y_{k}}$ number of controlled outputs.

Although, this block diagram representation retains the same form for all types of controllers (satisfying the rank constraints), the formulation of the optimization problem changes accordingly. In the given diagram, the controller can be a state-control, a measured output-control, or a static or dynamic feedback control. In addition, the order of the controller $\left(n_{K_{k}}\right)$, its time dependency (time-invariant or time-periodic), and if the controller is time-periodic its periodicity defines the problem formulated in Eq. 3.26. In this study, dynamic time-periodic controllers of order $n_{K_{k}}=n_{M_{k}}$ with a period equal to the period of the nominal system were designed. The state-space model of the block diagram in LFT form is given by the Eq. 3.24a, whereas the dynamic controller is given by the Eq. 3.24 b.

$$
\begin{gathered}
{\left[\begin{array}{l}
x_{k+1} \\
z_{k} \\
y_{k}
\end{array}\right]=\underbrace{\left[\begin{array}{c:cc}
A_{k} & B_{1_{k}} & B_{2_{k}} \\
\hdashline C_{1_{k}} & D_{11_{k}} & D_{12_{k}} \\
C_{2_{k}} & D_{21_{k}} & D_{22_{k}}
\end{array}\right]}_{M_{k}}\left[\begin{array}{l}
x_{k} \\
w_{k} \\
u_{k}
\end{array}\right]} \\
{\left[\begin{array}{l}
x_{K_{k+1}} \\
u_{k}
\end{array}\right]=\underbrace{\left[\begin{array}{ll}
A_{K_{k}} & B_{K_{k}} \\
C_{K_{k}} & D_{K_{k}}
\end{array}\right]}_{K_{k}}\left[\begin{array}{l}
x_{K_{k}} \\
y_{k}
\end{array}\right]}
\end{gathered}
$$

The closed-loop system shown in Fig. 3.3 has the closed-loop transfer function defined in terms of lower $\operatorname{LFT}\left(F_{L}\left(M_{k}, K_{k}\right)\right)$ as follows:

$$
F_{L}\left(M_{k}, K_{k}\right)=M_{11_{k}}+M_{12_{k}} K_{k}\left(I-M_{22_{k}}\right)^{-1} M_{21_{k}}=\left[\begin{array}{cc}
A_{c l_{k}} & B_{c l_{k}} \\
C_{c l_{k}} & D_{c l_{k}}
\end{array}\right]
$$

While both $H_{\infty}$ and $H_{2}$ controllers have to satisfy the stability of the closed-loop system, $F_{L}\left(M_{k}, K_{k}\right)$, the norm constraint on the closed-loop transfer function changes according to 
the controller.

\subsubsection{Discrete-Time Time-Periodic $H_{\infty}$ Controller Synthesis $[88,104,105$, 125]}

The objective of a suboptimal $H_{\infty}$ synthesis problem is to find a controller, $K_{\infty_{k}}$ that stabilizes the closed-loop system and gives to the infinite norm of the closed-loop transfer function between the disturbance, $w_{k}$ and error, $z_{k}$ signal a value less than some predefined parameter, $\gamma$. This condition is summarized as

$$
\begin{array}{ll}
\text { find } & K_{\infty_{k}} \\
\text { subject to } & \left\|F_{L}\left(M_{k}, K_{\infty_{k}}\right)\right\|_{\infty}<\gamma \\
& A_{c l_{k}}^{\prime} P_{k+1} A_{c l_{k}}-P_{k}<0 \\
& P_{k+N}=P_{k}
\end{array}
$$

The closed-loop state-space matrices given in Eq. 3.25 are defined by using the block diagonal operator, $\mathbb{\rrbracket}$ (for the definition of block diagonal operator see Appendix A):

$$
\begin{aligned}
& \left.\left.A_{c l_{k}}=\llbracket \begin{array}{cc}
A+B_{2} D_{K} C_{2} & B_{2} C_{K} \\
B_{K} C_{2} & A_{K}
\end{array}\right]_{k}, B_{c l_{k}}=\llbracket \begin{array}{c}
B_{1}+B_{2} D_{K} D_{21} \\
B_{K} D_{21}
\end{array}\right]_{k} \\
& C_{c l_{k}}=\llbracket C_{1}+D_{12} D_{K} C_{2} \quad D_{12} C_{K} \rrbracket_{k}, D_{c l_{k}}=\llbracket D_{11}+D_{12} D_{K} D_{21} \rrbracket_{k}
\end{aligned}
$$

which can be expressed as:

$$
\begin{aligned}
& A_{c l}=\bar{A}+\underline{B} K_{\infty} \underline{C}, \quad B_{c l}=\quad \bar{B}+\underline{B} K_{\infty} \underline{D}_{21} \\
& C_{c l}=\bar{C}+\underline{D}_{12} K_{\infty} \underline{C}, D_{c l}=\bar{D}_{11}+\underline{D}_{12} K_{\infty} \underline{D}_{21}
\end{aligned}
$$


where

$$
\begin{aligned}
& \left.\bar{A}=\llbracket\left[\begin{array}{cc}
A & 0 \\
0 & 0
\end{array}\right], \bar{B}=\llbracket\left[\begin{array}{c}
B 1 \\
0
\end{array}\right], \underline{B}=\llbracket\left[\begin{array}{cc}
0 & B_{2} \\
I & 0
\end{array}\right], K_{\infty}=\llbracket \llbracket \begin{array}{cc}
A_{K} & B_{K} \\
C_{K} & D_{K}
\end{array}\right] \\
& \left.\bar{C}=\llbracket\left[\begin{array}{ll}
C_{1} & 0
\end{array}\right], \underline{C}=\llbracket\left[\begin{array}{cc}
0 & I \\
C_{2} & 0
\end{array}\right], \underline{D}_{12}=\llbracket\left[\begin{array}{ll}
0 & D_{12}
\end{array}\right] \bar{D}_{21}=\llbracket \begin{array}{c}
0 \\
D_{21}
\end{array}\right]
\end{aligned}
$$

and the matrices $A, B_{1}, B_{2}, \cdots, C_{K}, D_{K}$ are defined in Eq. A.3:

$$
A=\left[\begin{array}{cccc}
A_{1} & & & \\
& A_{2} & & \\
& & \ddots & \\
& & & A_{N}
\end{array}\right], \cdots, \quad D_{K}=\left[\begin{array}{llll}
D_{K_{1}} & & & \\
& D_{K_{2}} & & \\
& & \ddots & \\
& & & D_{K_{N}}
\end{array}\right]
$$

The statement on the feasibility of obtaining a controller $K_{\infty}$ satisfying the stability and performance conditions stated in Eq. 3.26, is formulated by the following theorem, scaling $\gamma$ to 1 and assuming $D_{22_{k}}=0 \forall k=1, \cdots, N$ :

feasibility $X_{\infty}, Y_{\infty}$

subject to $\left[\begin{array}{cc}N_{c} & 0 \\ 0 & I\end{array}\right]^{*}\left[\begin{array}{ccc}Z A X_{\infty} A^{*} Z^{*}-X_{\infty} & Z A X_{\infty} C_{1}^{*} & Z B_{1} \\ C_{1} X_{\infty} A^{*} Z^{*} & C_{1} X_{\infty} C_{1}^{*}-I & D_{11} \\ B_{1}^{*} Z^{*} & D_{11}^{*} & -I\end{array}\right]\left[\begin{array}{cc}N_{c} & 0 \\ 0 & I\end{array}\right]<0$

$$
\begin{aligned}
& {\left[\begin{array}{cc}
N_{o} & 0 \\
0 & I
\end{array}\right]^{*}\left[\begin{array}{ccc}
A^{*} Z^{*} Y_{\infty} Z A-Y_{\infty} & A^{*} Z^{*} Y_{\infty} Z B_{1} & C_{1}^{*} \\
B_{1}^{*} Z^{*} Y_{\infty} Z A & B_{1}^{*} Z^{*} Y_{\infty} Z B_{1}-I & D_{11}^{*} \\
C_{1} & D_{11} & -I
\end{array}\right]\left[\begin{array}{cc}
N_{o} & 0 \\
0 & I
\end{array}\right]<0} \\
& {\left[\begin{array}{cc}
X_{\infty} & I \\
I & Y_{\infty}
\end{array}\right] \geq 0}
\end{aligned}
$$

where $\operatorname{Im}()$ and $\operatorname{ker}()$ define the image and the kernel of the matrix, respectively, $Z$ is 
the shift operator defined in Eq. 3.30.

$$
\begin{aligned}
& \operatorname{Im} N_{c}=\operatorname{ker}\left[\begin{array}{ll}
B_{2}^{*} Z^{*} & D_{12}^{*}
\end{array}\right], \quad N_{c} N_{c}^{*}=I \\
& \operatorname{Im} N_{o}=\operatorname{ker}\left[\begin{array}{ll}
C_{2} & D_{21}
\end{array}\right], \quad N_{o}^{*} N_{o}=I
\end{aligned} \quad \text { and } Z=\left[\begin{array}{cccc}
0 & \cdots & 0 & I \\
I & & 0 \\
& \ddots & \vdots \\
& I & 0
\end{array}\right]
$$

The search on the sub-optimal LTP $H_{\infty}$ controller that has the same period with the system model using LMIs (see Eq. 3.30), therefore requires

$$
N \underbrace{n_{M_{k}} \frac{n_{M_{k}}+1}{2}}_{X_{\infty_{k}}}+N \underbrace{n_{M_{k}} \frac{n_{M_{k}}+1}{2}}_{Y_{\infty_{k}}}
$$

number of decision variables associated with the $X_{\infty}, Y_{\infty}$ matrices. The solutions $X_{\infty}, Y_{\infty}$ are also block-diagonal and positive definite symmetric, as given in Eq. 3.32.

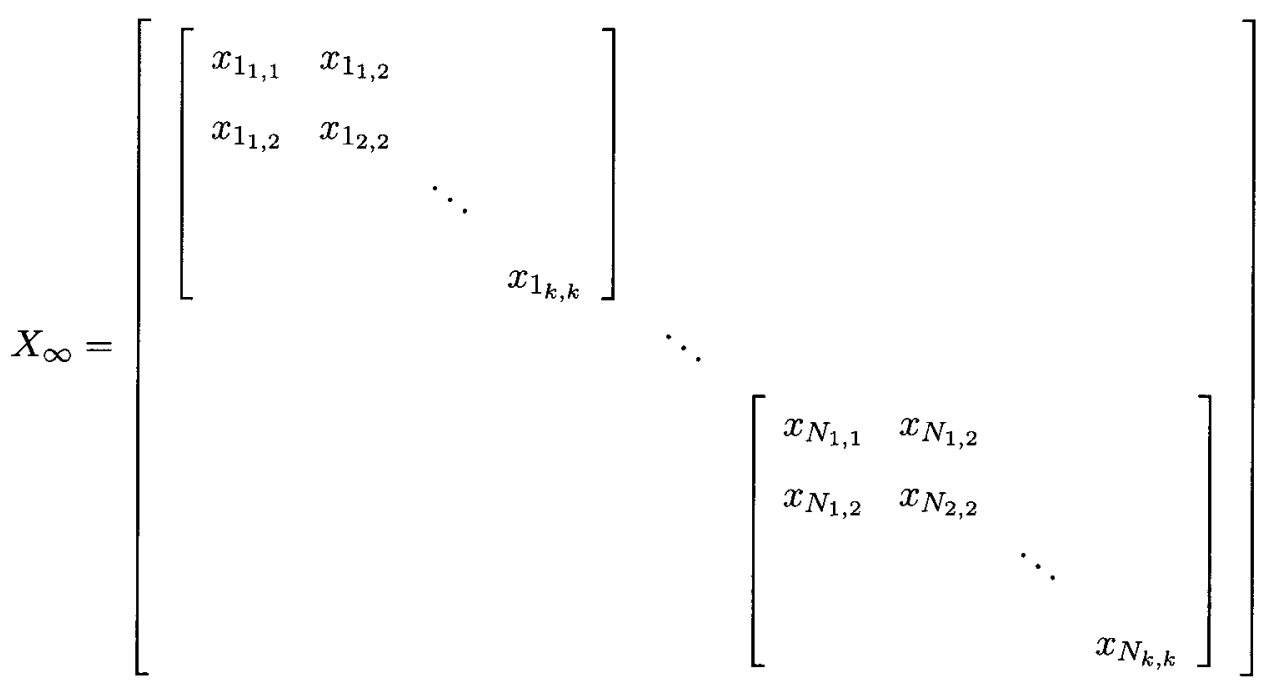

If the LMI conditions are found to be feasible, i.e. if there are periodic positive definite $X_{\infty}, Y_{\infty}$ matrices that satisfy the LMI constraints, then the controller's state-space matrices can be reconstructed using these matrices. The resultant controller is also time-periodic.

The controller's state-space matrices are reconstructed from the solutions $X_{\infty}, Y_{\infty}$ as follows. First, a matrix $X_{2}$ is defined by $Y_{\infty}-X_{\infty}^{-1}=X_{2} X_{2}^{*}$. Next, the $X_{c l}$ is defined 
using Eq. 3.33:

$$
X_{c l}=\llbracket\left[\begin{array}{cc}
Y_{\infty} & X_{2}^{*} \\
X_{2} & I
\end{array} \rrbracket, \quad X_{c l}^{-1}=\llbracket \begin{array}{cc}
X_{\infty} & -X_{\infty} X_{2}^{*} \\
-X_{2} X_{\infty} & I+X_{2} X_{\infty} X_{2}^{*}
\end{array}\right]
$$

The controller's state-space matrices are obtained by solving the feasibility problem on $K_{\infty}$, which satisfies the following LMI condition:

$$
\begin{array}{ll}
\text { feasibility } & K_{\infty} \\
\text { subject to } & H_{X_{c l}}+Q_{X_{c l}}^{*} K_{\infty}^{*} P_{X_{c l}}+P_{X_{c l}}^{*} K_{\infty} Q_{X_{c l}}<0
\end{array}
$$

where $K_{\infty}$ is used to represent the controllers state-space matrices, and the matrices $H_{X_{c l}}, Q_{X_{c l}}, P_{X_{c l}}$ are given in Eq. 3.35:

$$
\begin{array}{ll}
K_{\infty}=\left[\begin{array}{cc}
A_{K} & B_{K} \\
C_{K} & D_{K}
\end{array}\right], & H_{X_{c l}}=\left[\begin{array}{cccc}
-Z^{*} X_{c l}^{-1} Z & \bar{A} & \bar{B} & 0 \\
\bar{A}^{*} & -X_{c l} & 0 & \bar{C}^{*} \\
\bar{B}^{*} & 0 & -I & \bar{D}^{*} \\
0 & \bar{C} & \bar{D} & -I
\end{array}\right] \\
Q_{X_{c l}}=\left[\begin{array}{llll}
0 & \underline{C} & \underline{D}_{21} & 0
\end{array}\right], & P_{X_{c l}}=\left[\begin{array}{llll}
\underline{B}^{*} & 0 & 0 & \underline{D}_{12}
\end{array}\right]
\end{array}
$$

The controller matrix obtained from the optimization problem given in Eq. 3.34 has order $n_{K_{k}}=n_{M_{k}}$. The order of the controller can be decreased if necessary using periodic balanced order reduction [126]. Once the controller is designed, the stability and performance properties of the closed-loop system given in Eq. 3.28 are tested using a higher-order system model. In the final stage, the controller is validated by performing closed-loop control simulations on the full-order model.

\subsubsection{Discrete-Time Time-Periodic $\boldsymbol{H}_{2}$ Controller Synthesis $[48,50,89$, 110]}

The objective of an optimal $H_{2}$ synthesis problem is to find a controller, $K_{2_{k}}$ that stabilizes the closed-loop system and minimizes the $\mathrm{H}_{2}$ norm of the closed-loop transfer function 
between the disturbance, $w_{k}$ and error, $z_{k}$

$$
\begin{array}{ll}
\text { find } & K_{2_{k}} \\
\text { subject to } & \min \left\|F_{L}\left(M_{k}, K_{2_{k}}\right)\right\|_{2} \\
& \left|\lambda\left(\Psi_{c l_{k}}\right)\right|<1
\end{array}
$$

where the $\mathrm{H}_{2}$-norm of the time-periodic system (see Eq. 3.37b) can be calculated from the definition of controllability gramian, $P_{k}$ (see Eq. 3.37b) and observability gramian, $Q_{k}$ (see Eq. 3.37c) [48]:

$$
\begin{aligned}
\left\|F_{L}\left(M_{k}, K_{2_{k}}\right)\right\|_{2}^{2} & =\operatorname{trace}\left[\sum_{k=1}^{N} C_{c l_{k}} P_{k} C_{c l_{k}}^{\prime}+D_{c l_{k}} D_{c l_{k}}^{\prime}\right] \\
& =\operatorname{trace}\left[\sum_{k=1}^{N} B_{c l_{k}}^{\prime} Q_{k+1} B_{c l_{k}}+D_{c l_{k}}^{\prime} D_{c l_{k}}\right]
\end{aligned}
$$

where

$$
\begin{gathered}
P_{k+1}=A_{c l_{k}} P_{k} A_{c l_{k}}^{\prime}+B_{c l_{k}} B_{c l_{k}}^{\prime} \\
Q_{k}=A_{c l_{k}}^{\prime} Q_{k+1} A_{c l_{k}}+C_{c l_{k}}^{\prime} C_{c l_{k}}
\end{gathered}
$$

With some assumptions, this control problem can be written in terms of Riccati Equations. The assumptions are [50]

- Stabilizable $\left(A_{k}, B_{2_{k}}\right)$ and detectable $\left(C_{2_{k}}, A_{k}\right)$

- Non-singular $M_{k}=D_{12_{k}}^{\prime} D_{12_{k}}$ and $N_{k}=D_{21_{k}} D_{21_{k}}^{\prime}$

- Zero direct feed-through term $D_{22, k}=0$

The solution is based on two discrete-time TP Riccati Equations corresponding to the $H_{2}$ filtering problem (see Eq. 3.38a) and state-feedback control problem (see Eq. 3.38b) [110].

$$
\begin{aligned}
Y_{k+1}= & A_{k} Y_{k} A_{k}^{\prime}+B_{1_{k}} B_{1_{k}}^{\prime}-\left(A_{k} Y_{k} C_{2_{k}}^{\prime}+B_{1_{k}} D_{21_{k}}^{\prime}\right) \\
& \left(D_{21_{k}} D_{21_{k}}^{\prime}+C_{2_{k}} Y_{k} C_{2_{k}}^{\prime}\right)^{-1}\left(A_{k} Y_{k} C_{2_{k}}^{\prime}+B_{1_{k}} D_{21_{k}}^{\prime}\right)^{\prime} \\
X_{k}= & A_{k}^{\prime} X_{k+1} A_{k}+C_{1_{k}}^{\prime} C_{1_{k}}-\left(A_{k}^{\prime} X_{k+1} B_{2_{k}}+C_{1_{k}}^{\prime} D_{12_{k}}\right) \\
& \left(D_{12_{k}}^{\prime} D_{12_{k}}+B_{2_{k}}^{\prime} X_{k+1} B_{2_{k}}\right)^{-1}\left(A_{k}^{\prime} X_{k+1} B_{2_{k}}+C_{1_{k}}^{\prime} D_{12_{k}}\right)^{\prime}
\end{aligned}
$$


TP controller matrices are obtained from the positive definite symmetric solution of Riccati equations, $\left(P_{k}>0, Q_{k}>0\right)$ as [50]:

$$
\left[\begin{array}{c:c}
A_{K_{k}} & B_{K_{k}} \\
\hdashline C_{K_{k}} & D_{K_{k}}
\end{array}\right]=\left[\begin{array}{c:c}
A_{k}+B_{2_{k}} F_{k}-B_{2_{k}} L_{o_{k}} C_{2 k}+L_{k} C_{2_{k}} & B_{2_{k}} L_{o_{k}}-L_{k} \\
\hdashline F_{k}-L_{o_{k}} C_{2_{k}} & L_{o_{k}}
\end{array}\right]
$$

where,

$$
\begin{aligned}
& L_{k}=-\left(A_{k} Y_{k} C_{2_{k}}^{\prime}+B_{1_{k}} D_{21_{k}}^{\prime}\right)\left(D_{21_{k}} D_{21_{k}}^{\prime}+C_{2_{k}} Y_{k} C_{2 k}^{\prime}\right)^{-1} \\
& L_{o_{k}}=\left(F_{k} Y_{k} C_{2_{k}}^{\prime}+F_{o_{k}}+D_{21_{k}}^{\prime}\right)\left(D_{21_{k}} D_{21_{k}}^{\prime}+C_{2_{k}} Y_{k} C_{2_{k}}^{\prime}\right)^{-1} \\
& F_{k}=-\left(D_{12_{k}}^{\prime} D_{12_{k}}+B_{2_{k}}^{\prime} X_{k+1} B_{2_{k}}\right)^{-1}\left(B_{2_{k}}^{\prime} X_{k+1} A_{k}+D_{12_{k}}^{\prime} C_{1_{k}}\right) \\
& F_{o_{k}}=-\left(D_{12_{k}}^{\prime} D_{12_{k}}+B_{2_{k}}^{\prime} X_{k+1} B_{2_{k}}\right)^{-1}\left(B_{2_{k}}^{\prime} X_{k+1} A_{k}+D_{12_{k}}^{\prime} D_{11_{k}}\right)
\end{aligned}
$$

For non-zero direct feed-through term, $D_{22, k} \neq 0$, as in the case of blade pitch moment, the controller given in Eq. 3.39a is reconstructed. For this reconstruction, the matrix $Q_{D}=I+D_{K, k} D_{22, k}$ must be non-singular [89].

$$
\left[\begin{array}{c:c}
\hat{A}_{K_{k}} & \hat{B}_{K_{k}} \\
\hdashline \hat{C}_{K_{k}} & \hat{D}_{K_{k}}
\end{array}\right]=\left[\begin{array}{c:c}
A_{K_{k}}-B_{K_{k}} D_{22, k} Q_{D}^{-1} C_{K_{k}} & B_{K_{k}}\left(I-D_{22, k} Q_{D}^{-1} D_{K_{k}}\right) \\
\hdashline Q_{D}^{-1} C_{K_{k}} & Q_{D}^{-1} D_{K_{k}}
\end{array}\right]
$$

By substituting the controller matrices in Eq. 3.28, the closed-loop system is obtained (see Fig. 3.3). Then the stability the closed-loop system is analyzed by looking at the characteristic multipliers of $F_{L}\left(M_{k}, K_{k}\right)$. The performance of the controller was analyzed with the closed-loop control simulations.

\subsection{Solution of Discrete-Time Periodic Riccati Equation}

An alternative method to solve an optimal control problem $\left(H_{2}, H_{\infty}\right)$ is based on Riccati Equations [106]. Although many software packages such as Matlab provide a solution to time-invariant Riccati equations, there is no accessible algorithm for the solution of time-periodic Riccati Equations at the moment. Therefore, we attempted to develop an in- 
house discrete-time periodic Riccati Equation solver. The method is based on generalized eigenvalue problems and deflating subspaces and summarized as follows [106-111].

A unique $N$-periodic stabilizing solution $\left(X_{k}=X_{k}^{T} \geq 0, X_{k}=X_{k+N}\right)$ of discrete-time periodic Riccati Equation of the form [82]:

$$
\begin{aligned}
X_{k}= & A_{k}^{\prime} X_{k+1} A_{k}+C_{1_{k}}^{\prime} C_{1_{k}}-\left(A_{k}^{\prime} X_{k+1} B_{2_{k}}+C_{1_{k}}^{\prime} D_{12_{k}}\right) \\
& \left(D_{12_{k}}^{\prime} D_{12_{k}}+B_{2_{k}}^{\prime} X_{k+1} B_{2_{k}}\right)^{-1}\left(A_{k}^{\prime} X_{k+1} B_{2_{k}}+C_{1_{k}}^{\prime} D_{12_{k}}\right)^{\prime}
\end{aligned}
$$

can be formulated as a generalized eigenvalue problem, by defining the matrix pair formed with the states and the co-states of the system [82]:

$$
L_{k}\left[\begin{array}{c}
x_{k+1} \\
p_{k+1}
\end{array}\right]=M_{k}\left[\begin{array}{l}
x_{k} \\
p_{k}
\end{array}\right]
$$

where

$$
\begin{aligned}
L_{k} & =\left[\begin{array}{ll}
I & B_{2_{k}}\left(D_{12_{k}}^{\prime} D_{12_{k}}\right)^{-1} B_{2_{k}}^{\prime} \\
0 & \left(A_{k}-B_{2_{k}}\left(D_{12_{k}}^{\prime} D_{12_{k}}\right)^{-1} C_{1_{k}}\right)^{\prime}
\end{array}\right] \in R^{2 n \times 2 n} \\
M_{k} & =\left[\begin{array}{ll}
A_{k}-B_{2_{k}}\left(D_{12_{k}}^{\prime} D_{12_{k}}\right)^{-1} C_{1_{k}} & 0 \\
-C_{1_{k}}^{\prime}\left(I-D_{12_{k}}\left(D_{12_{k}}^{\prime} D_{12_{k}}\right)^{-1} D_{12_{k}}^{\prime}\right) C_{1_{k}} & I
\end{array}\right] \in R^{2 n \times 2 n}
\end{aligned}
$$

The matrix pair $\left(L_{k}, M_{k}\right)$ has $2 n$ characteristic multipliers and they are symmetric around the unit circle. For the systems with pure delays, there are zero characteristic multiplies and the corresponding infinite characteristic multipliers as shown in Eq. 3.43 [110,127]:

$$
\underbrace{0, \cdots, 0}_{r}, \underbrace{\lambda_{r+1}, \cdots, \lambda_{n}}_{n-r}, \underbrace{\frac{1}{\lambda_{n}}, \cdots, \frac{1}{\lambda_{r+1}}}_{n-r}, \underbrace{\infty, \cdots, \infty}_{r}
$$

with

$$
0<\left|\lambda_{\imath}\right|<1, \quad i=r+1, \cdots, n
$$

The $N$-periodic stabilizing solution, $X_{k}$ of Eq. 3.42 can be obtained from the basis of stable deflating subspaces by applying an ordered generalized real Schur form as outlined in [110]. 
In the first step, $N$-periodic orthogonal matrices, which triangularize the system associated with the generalized cyclic eigenvalue problem, are calculated [82]. In the second step, a re-ordering scheme is applied to have the eigenvalues of the monodromy matrix $\Psi_{T_{11}^{-1} H_{11}}$ within the unit circle. The resultant orthogonal matrices $Q_{k}$ and $Z_{k}$ give the $N$-periodic stabilizing solution, $X_{k}$ of Eq. 3.42:

$$
\begin{gathered}
Q_{k} L_{k} Z_{k+1}=\left[\begin{array}{cc}
T_{k, 11} & T_{k, 12} \\
0 & T_{k, 22}
\end{array}\right], \quad Q_{k} M_{k} Z_{k}=\left[\begin{array}{cc}
H_{k, 11} & H_{k, 12} \\
0 & H_{k, 22}
\end{array}\right] \\
X_{k}=Z_{k, 21} Z_{k, 11}^{-1}
\end{gathered}
$$

where

$$
Z_{k}=\left[\begin{array}{ll}
Z_{k, 11} & Z_{k, 12} \\
Z_{k, 21} & Z_{k, 22}
\end{array}\right]
$$

Since $L_{k}$ is not regular, the periodic Schur decomposition is applied [128]. The periodic Schur decomposition results in a matrix pair $L_{k}, M_{k}$ being in generalized periodic real Schur form. The transformation matrices are stored in the form $Q_{k}^{1}, Z_{k}^{1}$. The eigenvalues of the monodromy matrix are located on the diagonals as $1 \times 1$ blocks (real) and/or $2 \times 2$ blocks (complex conjugates) [128]. The re-ordering scheme is applied to sort the eigenvalues of the monodromy matrix so that the stable eigenvalues are located at the upper left corner. The re-ordering scheme is based on swapping the $1 \times 1$ and $2 \times 2$ blocks by finding the periodic orthogonal transformations $U_{k}$ and $V_{k}$ [129]. The orthogonal transformation matrices are obtained from the solution of periodic generalized (coupled) Sylvester equations, which are written in the Kronecker product form [129]. The swapping of each block continues until all the stable eigenvalues of the monodromy matrix are located at the upper left corner. The orthogonal transformation matrices are stored in the form $Q_{k}^{2}, Z_{k}^{2}$. The details of the reordering of eigenvalues of periodic matrix pairs are shown in the reference publication [129].

The resultant transformation matrix, $X_{k}$ that gives the $N$-periodic stabilizing solution to discrete-time $N$-periodic Riccati Equation is obtained from the accumulated and partitioned 
orthogonal transformation matrices:

$$
\begin{gathered}
Z_{k}=Z_{k}^{1} Z_{k}^{2}=\left[\begin{array}{cc}
Z_{k, 11} & Z_{k, 12} \\
Z_{k, 21} & Z_{k, 22}
\end{array}\right] \\
X_{k}=Z_{k, 21} Z_{k, 11}^{-1}
\end{gathered}
$$

The $N$-periodic positive definite solutions $Y_{k}, X_{k}$ of $H_{2}$ filtering (Eq. 3.38a) and $H_{2}$ control problem (Eq. 3.38b), respectively are used to form the $H_{2}$ controller matrix as summarized in Eq. 3.39 . 


\section{Chapter 4}

\section{Identification of Helicopter Rotor Blade Aeroelastic Response}

\subsection{Introduction}

This chapter presents details of the identification procedure that results in a model of the helicopter rotor system's aeroelastic response in a forward flight regime caused by trailing edge flap deflections. First, the system identification as a design problem is analyzed. Then, in Section 4.3, the input/output relationship is used to show the linearity and the periodicity of the helicopter rotor system's aeroelastic response around the operational condition. In Sections 4.5 and 4.6, discrete-time time-periodic state-space realization of the helicopter rotor system's aeroelastic response using subspace identification is presented. Two different system models are obtained and they are validated for several trailing edge flap deflection scenarios. The last section of this chapter, the effect of TEF deflection on the blade section aerodynamic properties is discussed.

\subsection{System Identification as a Design Problem: Input/output Selection}

The discrete-time time-periodic state-space model is used to design a controller to alleviate the helicopter rotor system's vibratory hub loads $\left(F_{X, Y, Z}, M_{X, Y, Z}\right)$ using TEFs. The hub loads are defined in the non-rotating frame and they are obtained from the summation of 
the transformed rotating frame blade loads of all blades in the non-rotating frame (Fig. 4.1). By using TEFs, the rotating frame blade loads can be altered as a result of the change in

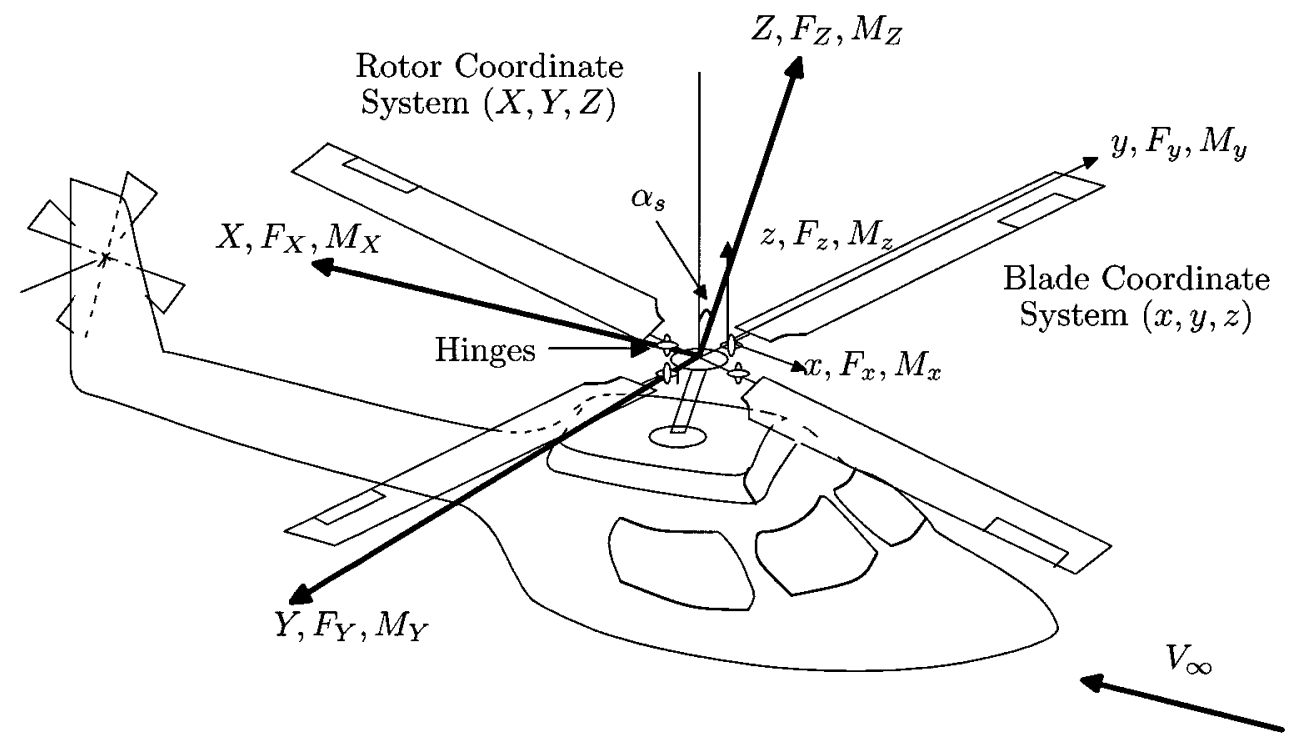

Figure 4.1: Definition of rotor and blade axis of reference in GENUVP/GAST (repeated).

the effective camber of the helicopter blade section. Since each blade contributes to the hub loads by controlling the blade loads of each blade at particular frequencies, the vibratory hub loads can be suppressed. Therefore, we chose to identify the blade loads and use them in the controller design. The reasons for this choice (Individual Blade Control) are as follows.

1. To analyze the direct influence of the TEFs:

In the rotating frame, blade loads have a rich frequency content. However, hub loads have only $n N_{b} / r e v, n=1,2, \cdots$ frequency content vibrations (see Section 2.3.2). This is because, the transformation of blade loads from rotating frame to non-rotating frame and their summation has a filtering effect caused by the phase difference between them. To analyze the direct influence of the TEF deflection, the rotating frame blade loads were analyzed before they were filtered.

2. To increase flexibility in control implementation: 
Helicopter vibration suppression using TEFs is an IBC approach, where each blade is equipped with its own control system. This approach provides flexibility in the hardware instrumentation in real-time applications, such as not installing TEFs to all blades.

3. To analyze the interactions of the blades through the disturbed wake:

The TEF deflection has the greatest influence on the blade loads of the blade that the TEF is located. However, the advancing blades are influenced by the deflection of the TEF of the preceding blades through the disturbed wake. To investigate this interaction, the blade loads under the effect of TEF deflection were identified.

TEF deflection does not change the rotating blade loads at a same amount in all coordinate directions $\left(F_{x, y, z}, M_{x, y, z}\right)$. First, to define the TEF actuation authority on the helicopter rotor system's aeroelastic response, physically realizable random deflections were imposed on the TEF and both the hub loads and the rotating frame blade loads were analyzed.

\subsubsection{Sensitivity Analysis: Effect of TEF Deflection on the Hub Loads and Blade Loads}

The TEF of one blade was deflected with a frequency bounded random signal as shown in Fig. 4.2. The effect of this deflection on to the total aeroelastic response in terms of hub loads and blade loads in all directions are given in Fig. 4.3 and Fig. 4.4 respectively.

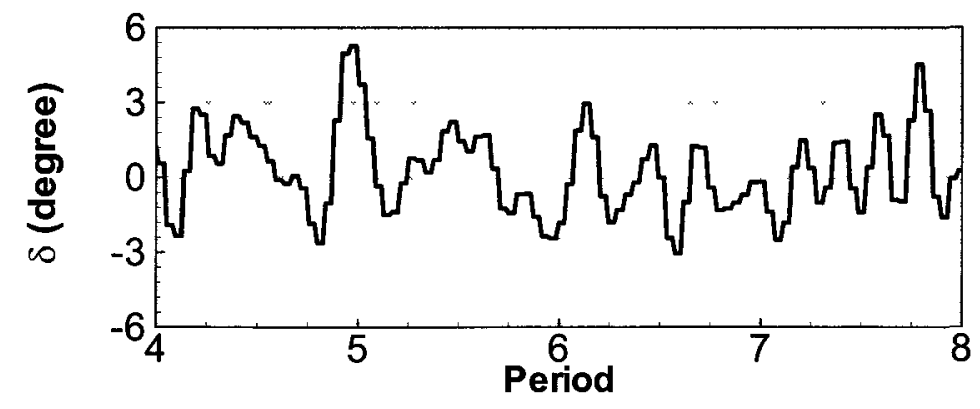

Figure 4.2: Physically realizable TEF deflection, $\delta_{1}$. 

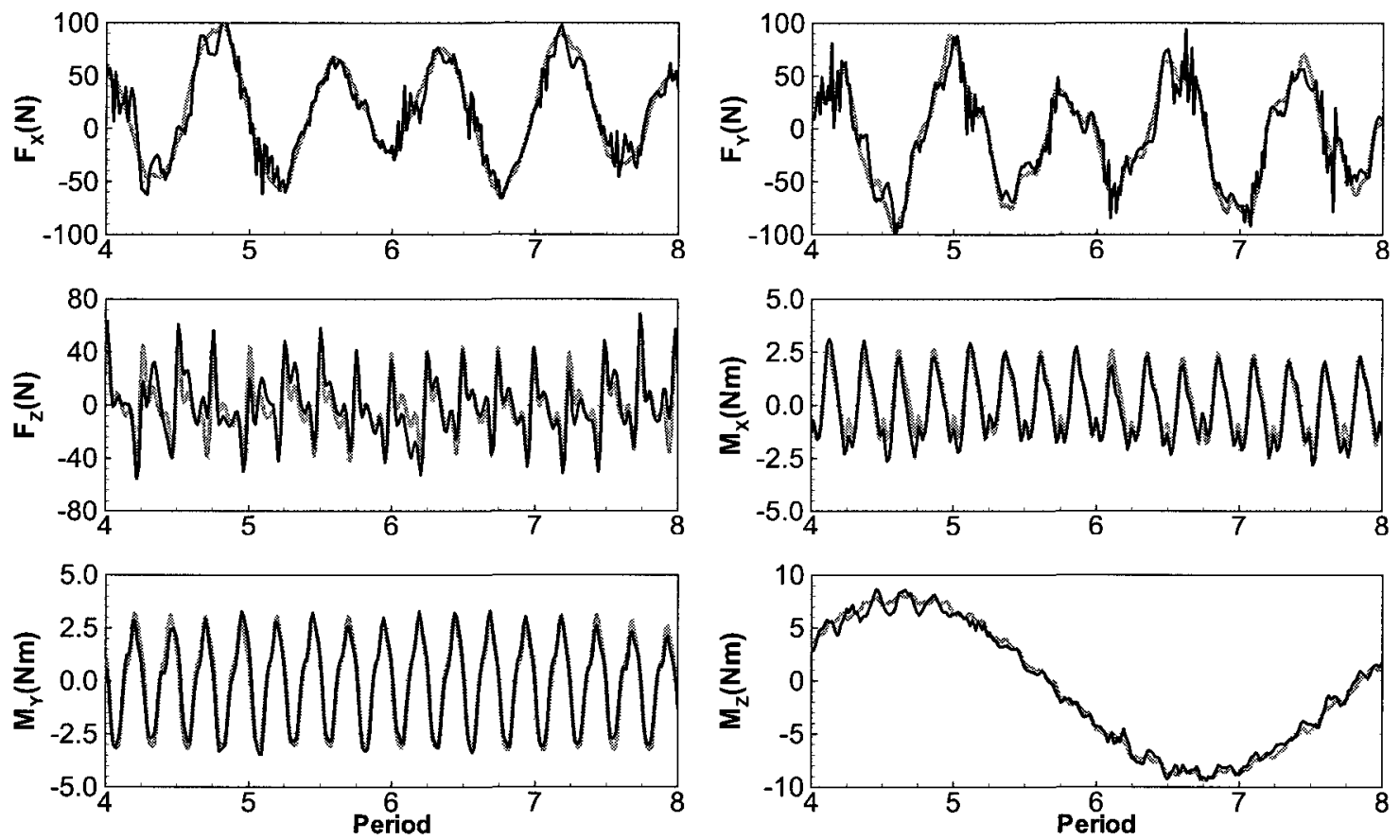

\section{Baseline}

Random TEF Deflection

Figure 4.3: Comparison of the helicopter rotor system's aeroelastic response in terms of hub loads, $F_{X}, F_{Y}, F_{Z}$ and moments, $M_{X}, M_{Y}, M_{Z}$ to the physically realizable TEF deflection $\delta_{1}$. 

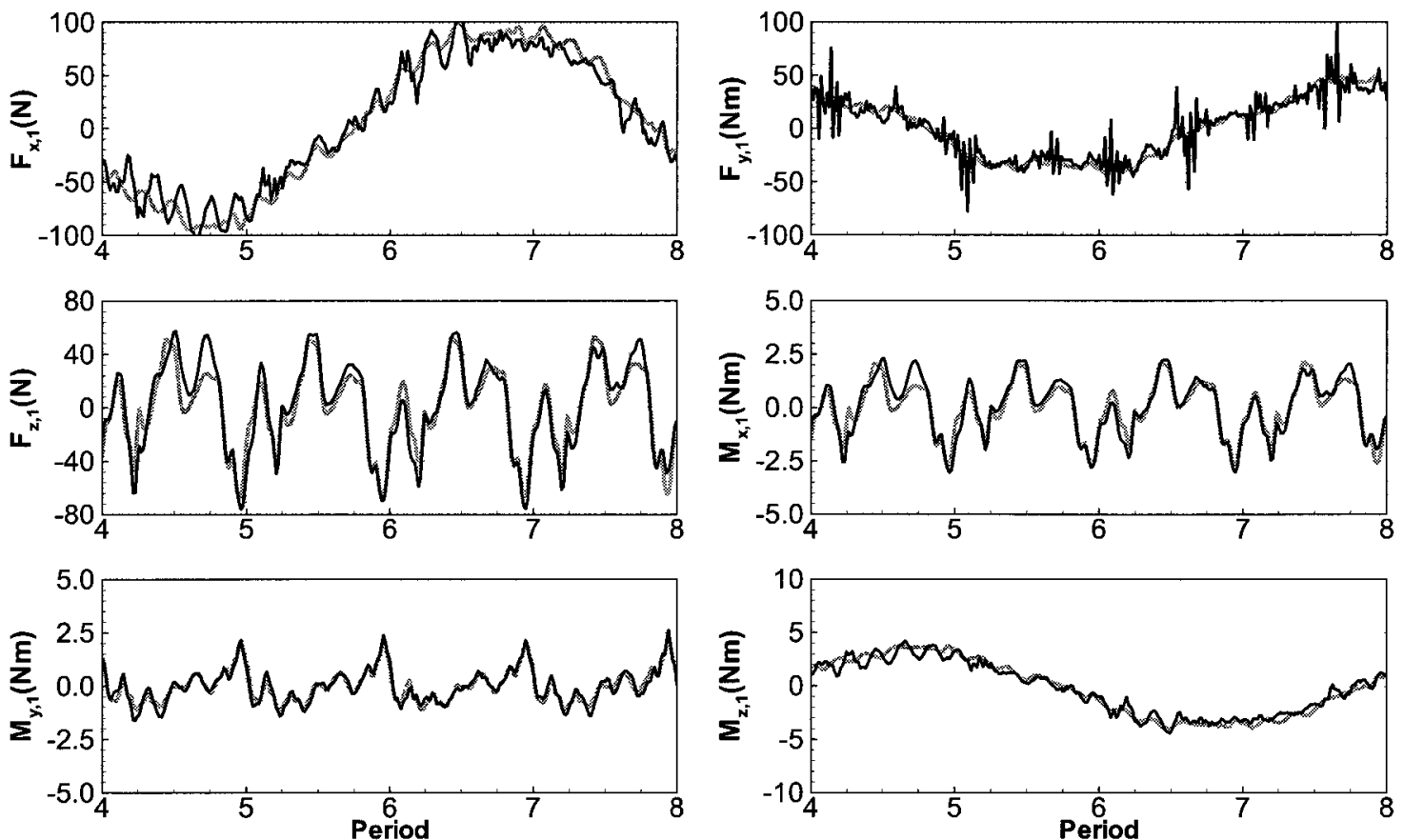

\section{Baseline \\ Random TEF Deflection}

Figure 4.4: Comparison of the helicopter rotor system's aeroelastic response in terms blade loads $F_{x, 1}, F_{y, 1}, F_{z, 1}$ and moments $M_{x, 1}, M_{y, 1}, M_{z, 1}$ to the physically realizable TEF deflection $\delta_{1}$. 
The average influence of the TEF deflection of one blade on the blade loads measured at all blades, and the hub loads were obtained in terms of relative $L_{2}$ norm (see Eq. 4.1) compared to the baseline vibrations. For physically realizable TEF deflections in the frequency range of $0-5 / \mathrm{rev}$ with maximum amplitude of $6^{\circ}$, the change in the overall response around the baseline in the frequency range of $0-10 / r e v$ is tabulated in Table 4.1.

$$
\text { The Relative Percentage } L_{2} \text { Norm } \equiv \sqrt{\frac{\sum_{\imath=1}^{N t}\left(F_{\imath}(\delta \neq 0)-F_{\imath}(\delta=0)\right)^{2}}{\sum_{\imath=1}^{N t} F_{\imath}(\delta=0)^{2}}} \times 100
$$

Table 4.1: Sensitivity of TEF deflection on the blade loads and hub loads in terms of relative percentage $L_{2}$ norm.

\begin{tabular}{|c|c|c|c|c|c|c|}
\hline Hub Loads & $F_{X}$ & $F_{Y}$ & $F_{Z}$ & $M_{X}$ & $M_{Y}$ & $M_{Z}$ \\
$L_{2}(\%)$ norm & 19.8 & 20.2 & 66.7 & 30.2 & 19.3 & 7.2 \\
\hline \hline Blade Loads & $F_{x, 1}$ & $F_{y, 1}$ & $F_{z, 1}$ & $M_{x, 1}$ & $M_{y, 1}$ & $M_{z, 1}$ \\
$L_{2}(\%)$ norm & 16.6 & 19.8 & 41.5 & 41.5 & 30 & 16.6 \\
\hline \hline Blade Loads & $F_{x, 2}$ & $F_{y, 2}$ & $F_{z, 2}$ & $M_{x, 2}$ & $M_{y, 2}$ & $M_{x, 2}$ \\
$L_{2}(\%)$ norm & 2.0 & 1.5 & 4.3 & 4.3 & 3.5 & 2.0 \\
\hline \hline Blade Loads & $F_{x, 3}$ & $F_{y, 3}$ & $F_{z, 3}$ & $M_{x, 3}$ & $M_{y, 3}$ & $M_{z, 3}$ \\
$L_{2}(\%)$ norm & 4.5 & 3.5 & 7.6 & 7.6 & 5.6 & 4.5 \\
\hline \hline Blade Loads & $F_{x, 4}$ & $F_{y, 4}$ & $F_{z, 4}$ & $M_{x, 4}$ & $M_{y, 4}$ & $M_{z, 4}$ \\
$L_{2}(\%)$ norm & 5.2 & 5.0 & 15.3 & 15.3 & 10.8 & 5.2 \\
\hline
\end{tabular}

The TEF deflection has a considerable influence on the trust, $F_{Z}$ the helicopter rotor system's hub roll moment, $M_{X}$ and hub pitch moment $\left(M_{Y}\right)$. However, the influence on the hub yaw moment, $M_{Z}$ caused by the rigid lead-lag motion of the blade at $0.2 /$ rev is not noticeable. A similar result was observed when the blade loads were analyzed and presented in Fig. 4.4. Therefore, controlling the blade's rigid lead-lag motion with TEF, and eventually the vibratory hub load, $M_{Z}$, with the use of TEFs is not feasible in the specific simulations we carried out. Since, in the calculations drag correction was omitted. The only influence 
on the blade lead-lag is caused by the coupling between the blade flapping and lead-lag through the pre-twist. However, the TEF could potentially reduce the hub vibratory loads in the vertical direction, $F_{Z}$ and roll and pitch moments, $M_{X}$ and $M_{Y}$, respectively. For the hub loads $F_{X}$ and $F_{Y}$, the TEF influence is also noticeable. However, this influence was mostly observed at the higher frequencies. The suppression of the vibration at the low frequency region is also questionable. In Table 4.1, the relative effect of the TEF deflection of one blade on the other blades aeroelastic response is also given. While the blade that is in the wake of the deflected TEF experiences the highest influence of the change in the flow field, the other blades experience slight change caused by the disturbance of the flow.

Based on the sensitivity analysis, by deflecting the TEFs, the hub loads $F_{Z}, M_{X}$ and $M_{Y}$ can be altered. In order to design a controller that suppress these vibratory hub loads while performing individual blade analysis, we chose to identify a map between the TEF deflection of one blade and the rotating frame blade loads $F_{z}$ and $M_{y}$. As a first step in the identification, properties of this map were analyzed around the baseline condition. Since only the effect of the TEF deflection on the aeroelastic responses in terms of rotating frame blade loads has to be considered, the baseline vibrations presented in Section 2.3.2 was subtracted from the total aeroelastic response. The resultant system identification set up for the controller design and analysis is summarized in Fig. 4.5. 


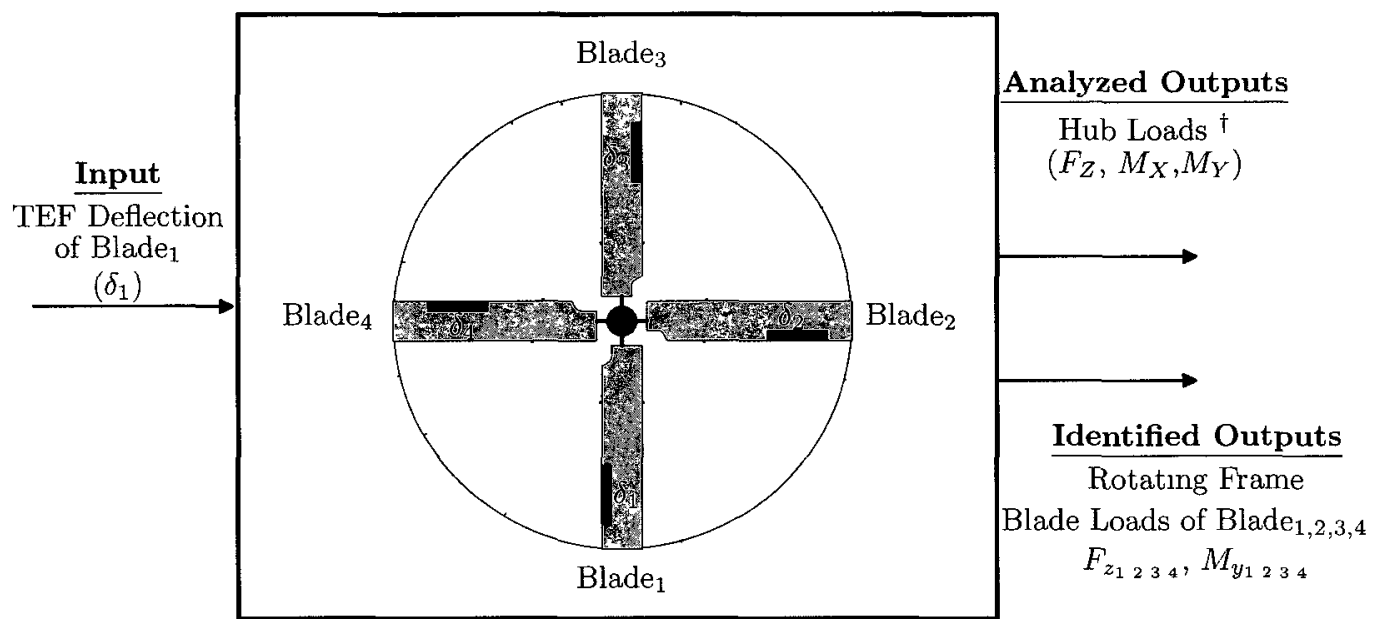

( ${ }^{\dagger}$ Baselıne loads were substracted)

Figure 4.5: Identification setup for the helicopter rotor system's aeroelastic response under TEF deflection.

\subsection{Linearity, Periodicity, and Equivalency of the Helicopter Rotor Blade's Aeroelastic Response}

The helicopter rotor system is exposed to highly complex aerodynamic loading that is caused by a combination of several aerodynamic phenomena (see Chapter 1 ). Under this complex aerodynamic and inertial loading, the rotor blade undergoes large elastic deformations. As a result, the resultant helicopter blade aeroelastic response in terms of hub loads and blade loads shows non-linear behavior in the complete flight envelope. Although the non-linearity is an inherent behavior of the complete flight envelope, it is necessary to determine if the helicopter rotor system's aeroelastic response caused by the TEF deflection can be modeled by a linear operator around a baseline flight regime. In other words, it is necessary to investigate if the helicopter rotor system's aeroelastic response has a linear dependency to the TEF deflection around the baseline flight regime. The baseline forward-flight regime and the trim state condition are presented in Section 2.3.2.

To investigate linearity around the baseline condition, several simulations were carried 
out by imposing a deflection to the TEF in the frequency and amplitude range of interest when the helicopter is flying in the baseline forward flight regime. For the sake of brevity and clarity, only one of the results is presented here. The trailing edge flap of the first blade was deflected with $\delta_{1,1}=2 \cos \left(2.7 \Psi_{1}\right)$ degrees, $\delta_{2,1}=4 \cos \left(2.7 \Psi_{1}\right)$ degrees and $\delta_{3,1}=\delta_{1,1}+\delta_{2,1}$ in three different simulations. Resultant blade loads on the first blade, caused by the TEF deflections $\delta_{1,1}, \delta_{2,1}$ and $\delta_{3,1}$ were called as outputs $y_{1,1}, y_{2,1}$ and $y_{3,1}$, respectively. To validate the linearity assumption, the output obtained from the linear combination of two separate outputs, $y_{1}=y_{1,1}+y_{2,1}$ and the output $y_{3,1}$ are presented in Fig. 4.6 and Fig. 4.7. 

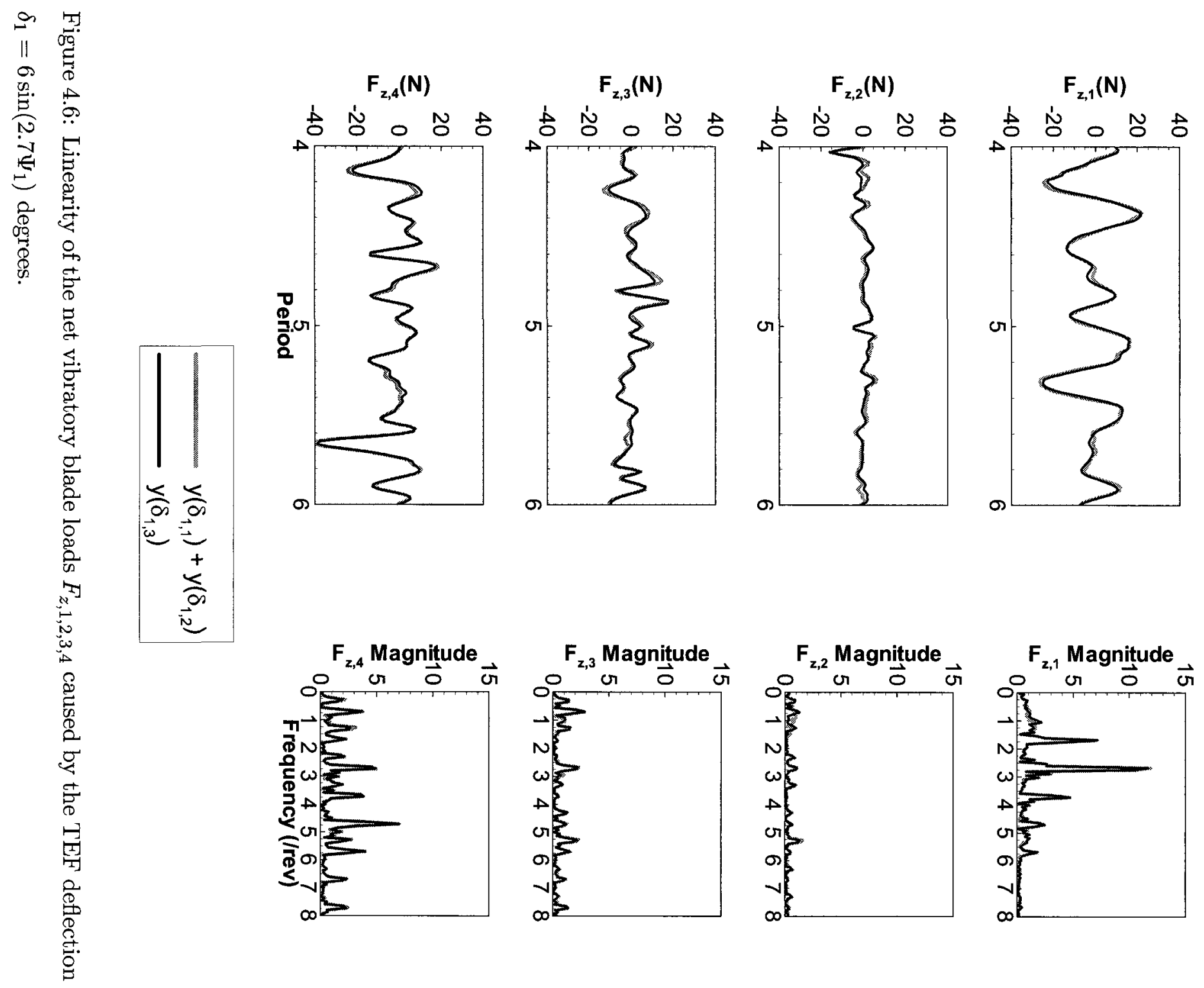

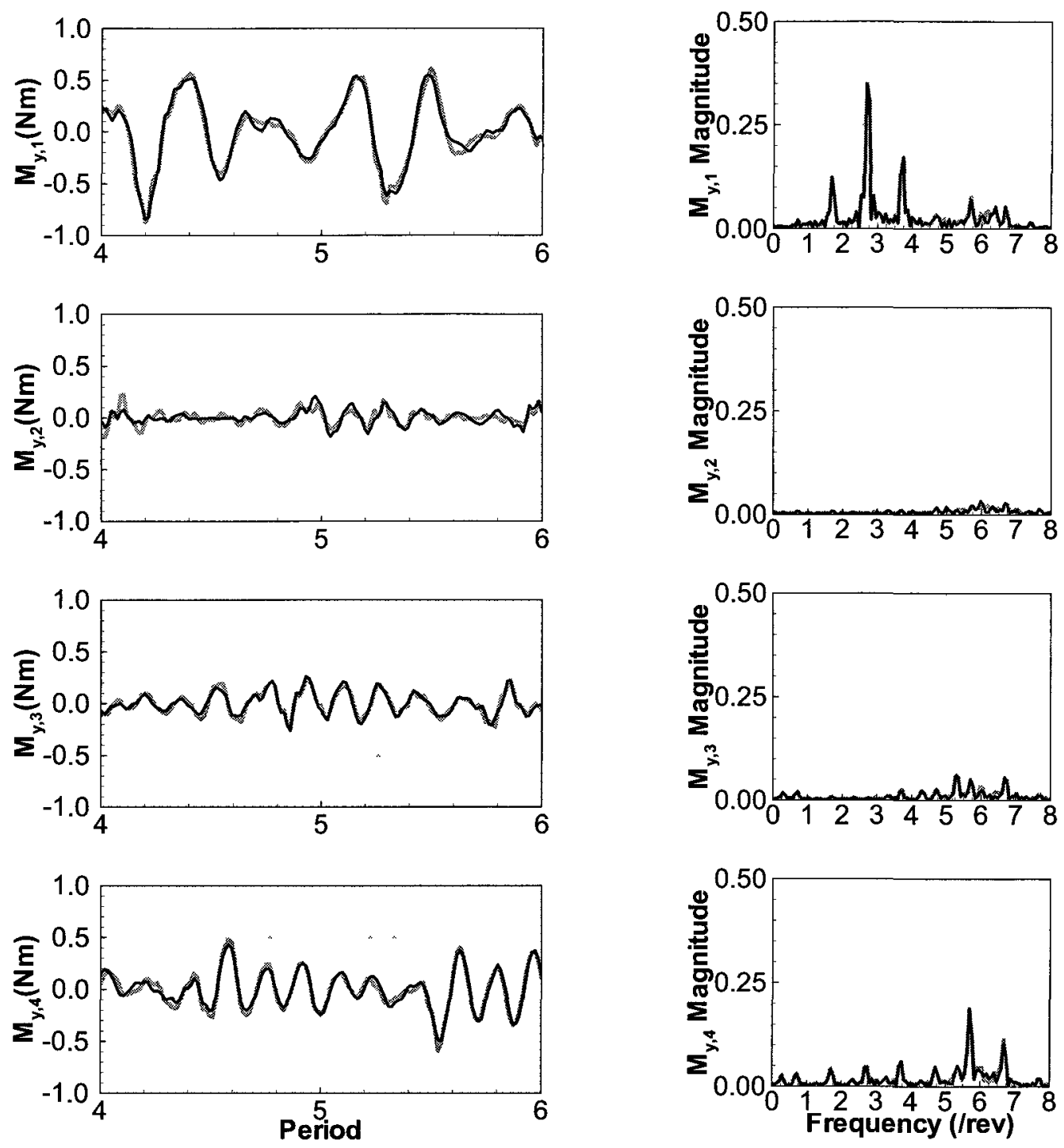

$$
\begin{aligned}
& y\left(\delta_{1,1}\right)+y\left(\delta_{1,2}\right) \\
& y\left(\delta_{1,3}\right)
\end{aligned}
$$

Figure 4.7: Linearity of the net vibratory blade loads $M_{y, 1,2,3,4}$ caused by the TEF deflection $\delta_{1}=6 \sin \left(2.7 \Psi_{1}\right)$ degrees.

The blade one loads $F_{z, 1}, M_{y, 1}$ caused by the deflection of the TEF on the same blade, $\delta_{3,1}=\delta_{1,1}+\delta_{2,1}$ are almost equal to the linear combination of the individual blade loads 
caused by the TEF deflections of $\delta_{1,1}$ and $\delta_{2,1}$ (direct effect). The same conclusion can be drawn when the blade loads of the other blades, $F_{z, 2,3,4}, M_{y, 2,3,4}$ are analyzed. In conclusion, the system response to the TEF deflection with the frequency of $2.7 /$ rev, with maximum amplitude of $\pm 6^{\circ}$, can be considered as linear around the operational baseline condition. This result will be assumed typical for the entire range of frequencies of interest, i.e., between $0-5 /$ rev.

It is a well known fact that the helicopter rotor system's aeroelastic response in forward flight regime, with high advance ratio, shows a time periodic behavior [3]. Time periodicity implies that the impulse response function is time-periodic $(g(t, \tau)=g(t+T, \tau+T))$. To validate the periodicity of the aeroelastic response, an impulsive input was given to the TEF of the first blade at azimuth angles $\Psi=90^{\circ}$ and $\Psi=270^{\circ}$ with an amplitude of $\delta_{1}=4^{\circ} \delta_{c}\left(\Psi-\Psi_{1}\right)$, and the blade loads of the first blade caused by the TEF deflections were compared (see Fig. 4.8). 

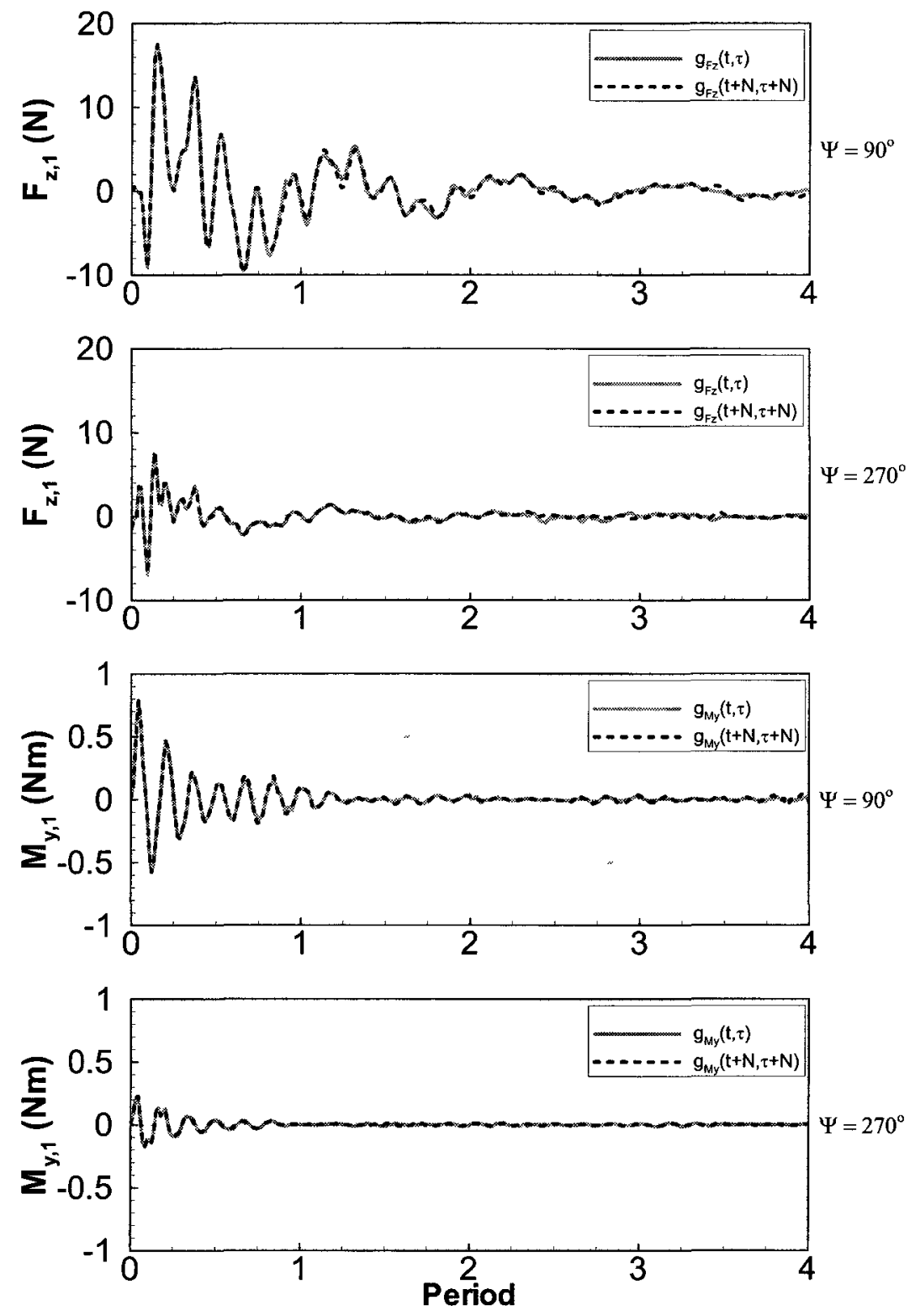

Figure 4.8: Comparison of helicopter rotor system's aeroelastic impulse responses in terms of blade load, $F_{z, 1}, M_{y, 1}$ to a TEF deflection of $4^{\circ}$ amplitude at azimuth locations $\Psi=90^{\circ}, \Psi=270^{\circ}$ and same azimuth location after a full revolution.

As the blade rotates, the aeroelastic response of the rotor blade to the impulsive TEF deflection varies significantly. Moreover, the impulse responses of the rotor blade to the TEF deflections applied at $\Psi=90^{\circ}$, and at the same azimuth angle after one full revolution are 
equivalent. This result confirms the time-periodic behavior of the helicopter rotor system's aeroelastic response in forward flight, $g(t, \tau)=g(t+T, \tau+T)$. In addition, Fig. 4.8 shows that when the blade is passing through the advancing side, around an azimuth angle $\Psi=90^{\circ}$, the aeroelastic response in terms of blade loads to the TEF deflection has its highest value for $F_{z, 1}, M_{y, 1}$. When it is passing through the retreating side, around an azimuth angle $\Psi=270^{\circ}$, it has its lowest value. This result was also expected since at the advancing side the velocity that the blade section experiences is higher. Accordingly, when the TEF is deflected at the advancing side, the amplitude of the impulsive force that the blade experiences caused by the TEF deflection is higher.

Another property of time periodic systems is analyzed in terms of the steady-state frequency responses (see Section 1.2.1). The frequency responses show that the steadystate helicopter rotor system's aeroelastic response in terms of the net rotating frame blade loads caused by the TEF deflection has a frequency content of not only the forcing frequency $\omega=2.7 / \mathrm{rev}$, but also integer multiples of rotation frequency $\omega \pm n \omega_{p}, n=0,1,2,3,4$ (see Fig. 4.6 and Fig. 4.7).

To determine the equivalency of the blades, both the structural and the aerodynamic properties of the blades were analyzed. The blades are considered structurally equivalent. However, at the baseline forward flight regime, due to the low-damping the lower-frequency lead-lag motion of the blades did not reach to the same steady-state value (Section 2.3.2). This difference leads to an aerodynamic dissimilarity due to the difference in the effective inflow velocity that each blade section experiences. To evaluate the influence of this dissimilarity, the impulse response of two blades was compared at two azimuth locations (see Fig. 4.9). 

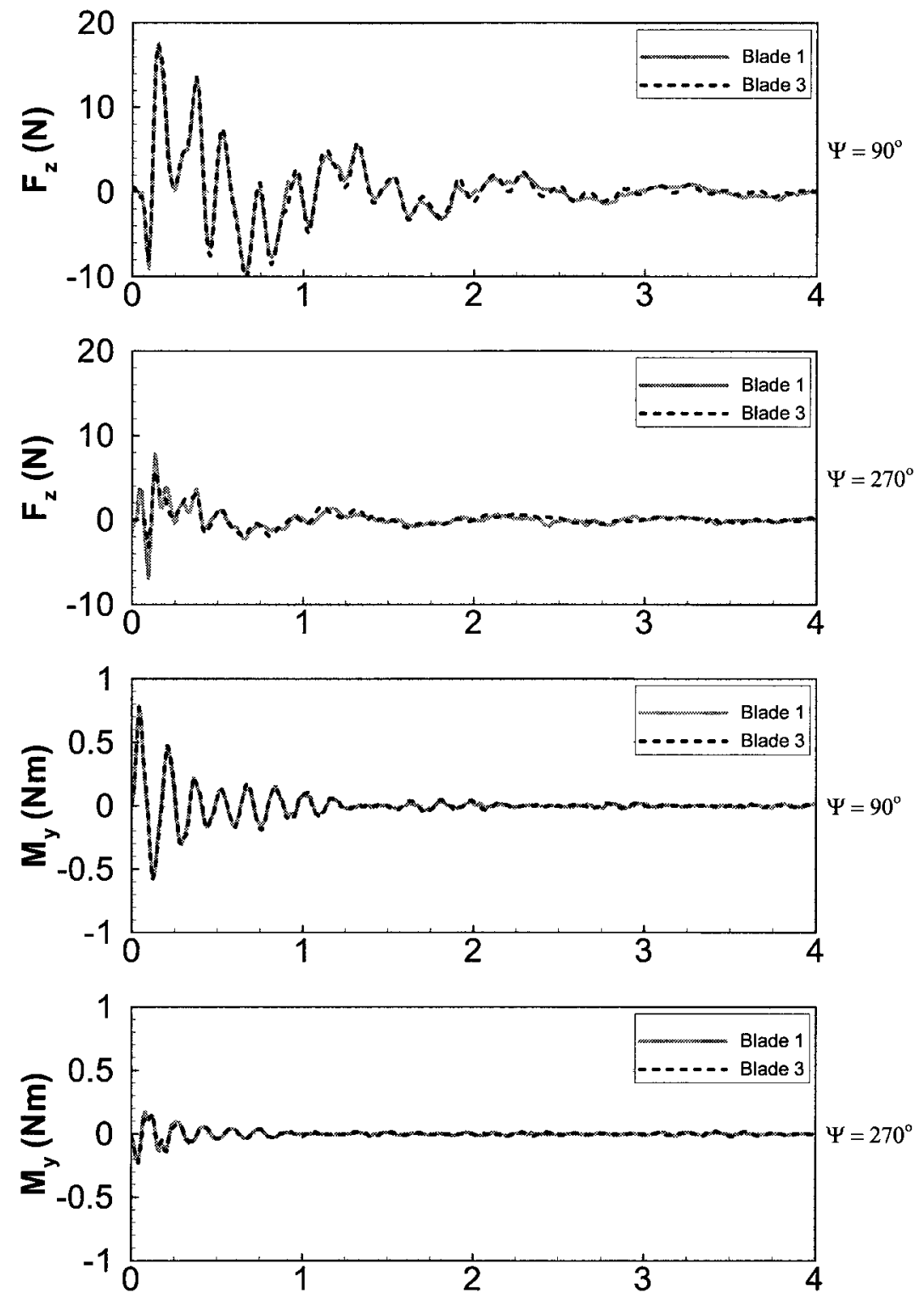

Figure 4.9: Comparison of aeroelastic impulse responses of the helicopter rotor system in terms of blade loads $F_{z, 1,3}, M_{y, 1,3}$ to a TEF deflection of $4^{\circ}$ amplitude at azimuth locations $\Psi=90^{\circ}, \Psi=270^{\circ}$.

Figure 4.9 shows that the impulse responses of blade one and three are very similar. The difference in the lead-lag motion of the blades did not cause significant dissimilarity in the blade's aeroelastic responses when the effect of the TEF deflection was analyzed. 
Therefore, the blades can be considered aeroelasticly equivalent.

\subsection{System Identification as a Design Problem: Model Set \& Simulation Design}

In this section, the GENUVP/GAST simulation for the identification of the LTP operator, which gives a map between the TEF deflection and the rotating frame blade loads $F_{z}$ and $M_{y}$, is summarized. The input/output map was chosen to be a discrete-time periodic state-space model as given in Eq. 4.2.

$$
\left[\begin{array}{c}
x_{k+1} \\
y_{k}
\end{array}\right]=\left[\begin{array}{cc}
A_{k} & B_{k} \\
C_{k} & D_{k}
\end{array}\right]\left[\begin{array}{l}
x_{k} \\
u_{k}
\end{array}\right]
$$

where,

$$
\begin{array}{ll}
A_{k+N}=A_{k} \in \mathbb{R}^{n_{k} \times n_{k}}, & B_{k+N}=B_{k} \in \mathbb{R}^{n_{k} \times m_{k}} \\
C_{k+N}=C_{k} \in \mathbb{R}^{p_{k} \times n_{k}}, & D_{k+N}=D_{k} \in \mathbb{R}^{p_{k} \times m_{k}}
\end{array}
$$

where, $n_{k}$ is the order of the system, not known a priori, $x_{k} \in \mathbb{R}^{n_{k}}, m_{k}$ is the number of inputs, $u_{k} \in \mathbb{R}^{m_{k}}$, and $p_{k}$ is the number of outputs, $y \in \mathbb{R}^{p_{k}}$. Equation 4.2 states that the LTP system is $N$-periodic.

Referring back to Fig. 4.5, the input is the TEF deflection of the first blade, $u=\delta_{1}, m_{k}=1 \forall k=1, \cdots N$. Two different analyses were performed concerning the output of the system. In the first analysis, the outputs of the system were taken as $y=F_{z, 1}, M_{y, 1} \in \mathbb{R}^{p_{k}}, p_{k}=2 \forall k=1, \cdots, N$, and the effect of the TEF deflection on the other blades was ignored. In the second analysis, the outputs of the system were taken as $y=F_{z, 1,2,3,4}, M_{y, 1,2,3,4} \in \mathbb{R}^{p_{k}}, p_{k}=8 \forall k=1, \cdots, N$. This includes the interaction of the blades through the disturbed wake. The resultant state-space model provides an input/output map between the TEF deflection of the first blade and the rotating frame blade loads of all blades.

In the GENUVP/GAST aeroelastic simulations, one full rotation was divided into twenty four equally spaced intervals as shown in Fig. 4.10. Physically realizable random deflections were given to the TEF of the first blade with a maximum amplitude of $5^{\circ}$ at 
each discrete azimuth location $\left(\Psi_{\{k=1,2,, 24\}}=0^{\circ}, 15^{\circ}, \cdots, 345^{\circ}, \Delta_{\Psi}=15^{\circ}\right)$. The TEF deflection along the interval $\Delta_{\Psi}=15^{\circ}$ was kept constant to satisfy the zero-order-hold assumption in the discretization. Since, the GENUVP/GAST required a better time resolution to solve for the helicopter rotor system's aeroelastic response, each azimuth interval $\Delta_{\Psi}$ was divided into three to have the sampling period of the GENUVP/GAST aeroelastic simulations being $\delta_{\Psi}=5^{\circ}$.

The (3-to-1) difference in the sampling period of the GENUVP/GAST $\left(\delta_{\Psi}\right)$ and the model $\left(\Delta_{\Psi}\right)$ shows a multi-rate sampling system behavior. To have a better resolution in the model, time-lifting was applied (Section 1.2.1). The outputs of the GENUVP/GAST aeroelastic code were packed as given in Eq. 1.3 to obtain the lifted outputs $\underline{F}_{z, \imath}$ and $\underline{M}_{y, 2}$. It should be noted that here the time-lifting technique was applied due to the multi-rate sampling of the GENUVP/GAST and the model, and not because the discrete-time statespace model is time-periodic. The resultant discrete-time time-periodic state-space model had a period $N=24$ with one input, $u=\delta_{1}, m_{k}=1 \forall k=1, \cdots 24$, and $8 \times 3$ outputs for the case of $y=\underline{F}_{z, 1,2,3,4}, \underline{M}_{y, 1,2,3,4}, p_{k}=24 \forall k=1, \cdots, 24$.

One important advantage of using the subspace identification method is to allow the use of an ensemble of data sets. To decrease the computational cost caused by the accumulation of vortex particles over time, which slows down the aeroelastic simulations, the identification simulations were carried out in twenty-six independent shorter period GENUVP/GAST aeroelastic runs. 


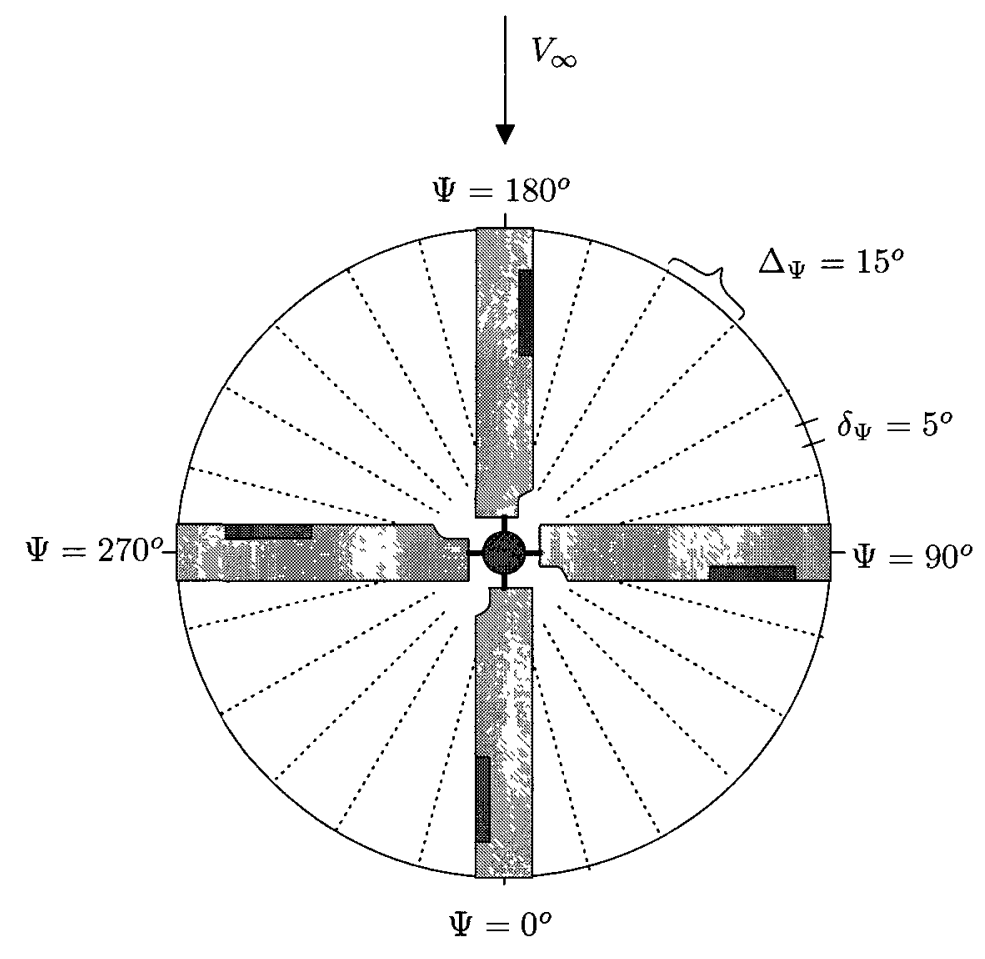

Figure 4.10: The GENUVP/GAST simulation setup for system identification.

\subsection{Discrete-Time Time-Periodic State-Space Realization us- ing Subspace Identification Part I: One Blade Analysis}

The ensemble of aeroelastic responses that resulted from physically realizable and persistently exciting TEF deflections was used to construct the generalized input/output Hankel matrices. By applying the MOESP algorithm, introduced in Section 3.4, the discrete-time time-periodic state-space model given in Eq. 4.3 was obtained for $k=1, \cdots, 24$.

$$
\left[\begin{array}{c}
x_{k+1} \\
y_{k}
\end{array}\right]=\left[\begin{array}{cc}
A_{k} & B_{k} \\
C_{k} & D_{k}
\end{array}\right]\left[\begin{array}{l}
x_{k} \\
u_{k}
\end{array}\right]
$$

The order of the system was determined from the singular values of the output Hankel 
Matrix, $R_{22, k}$ (Eq. 3.15) and from the comparison of the relative percentage $L_{2}$ error norm of the model outputs. The singular values of $R_{22, k}$ are given in Fig. 4.11.

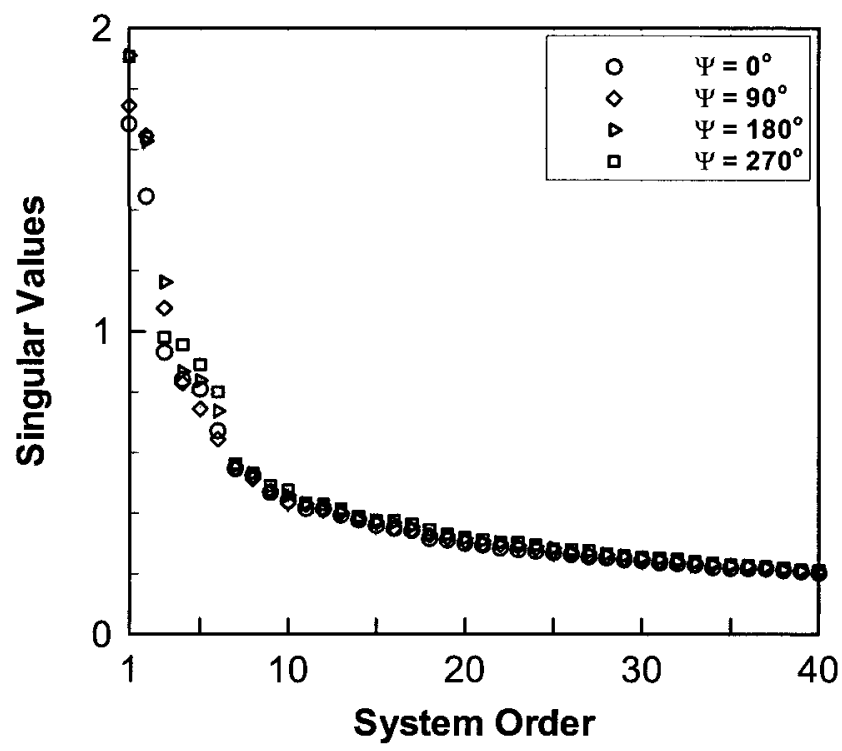

Figure 4.11: Singular Values of the output Hankel matrix $R_{22}$.

Figure 4.12 presents the relative percentage $L_{2}$ error norm between the blade loads $\underline{F}_{z, 1}$ and $\underline{M}_{y, 1}$ obtained from the GENUVP/GAST simulations and from the system model of different orders, under the physically realizable random TEF deflections of one blade. It should be noted that the TEF deflection used for error comparison purposes was not used in the identification process. 


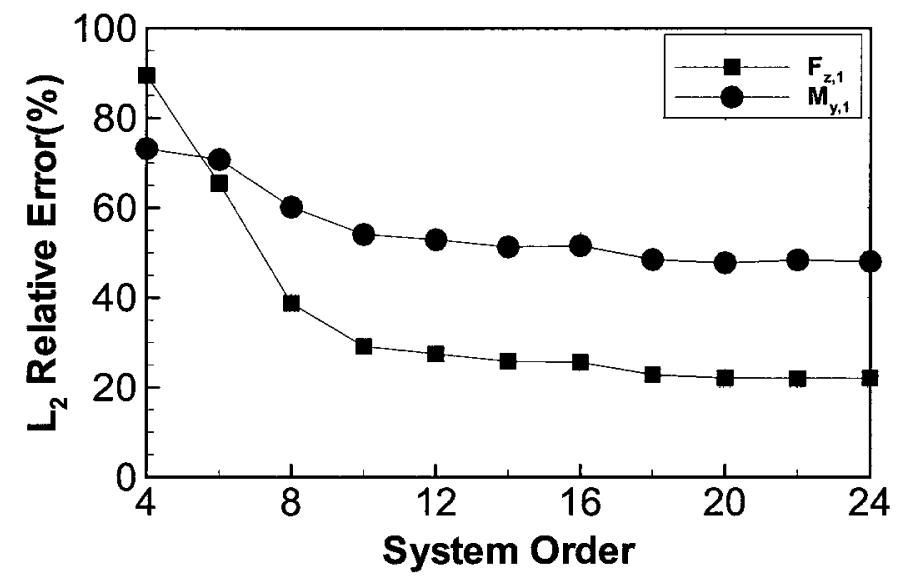

Figure 4.12: Relative percentage $L_{2}$ error norm between the blade loads $F_{z, 1}, M_{y, 1}$ obtained from the GENUVP/GAST simulation and system model of different orders.

As seen from Fig. 4.12, while the order of the system model was increased, the error decreased between the GENUVP/GAST simulations and the model. However, for the system model having an order above $n=12$, there was no significant improvement in predicting the actual response. In addition, the distribution of the singular values for each azimuth angle was almost the same above order 12. Therefore, the system order was chosen to be $n_{k}=12, \forall k=1, \cdots, 24$.

\subsubsection{Stability of the System}

The stability of the discrete-time LTP state-space model of the helicopter rotor system's aeroelastic response in terms of the rotating frame blade loads $\underline{F}_{z}$ and $\underline{M}_{y}$ caused by the TEF deflection was analyzed using Floquet Theory (see Section 3.5). The distribution of the eigenvalues of the system at different azimuth angles and the characteristic multipliers (eigenvalues of the monodromy matrix, $\Psi_{k}$ ) are presented in Fig. 4.13. At each azimuth angle the system has poles in very close vicinity of unit disk. However, we can not judge on the stability of the system based on the eigenvalues at individual azimuth locations. For LTP systems, the characteristic multipliers define the stability of the system. Since for our system they lie in the unit circle, the system is considered to be stable. 

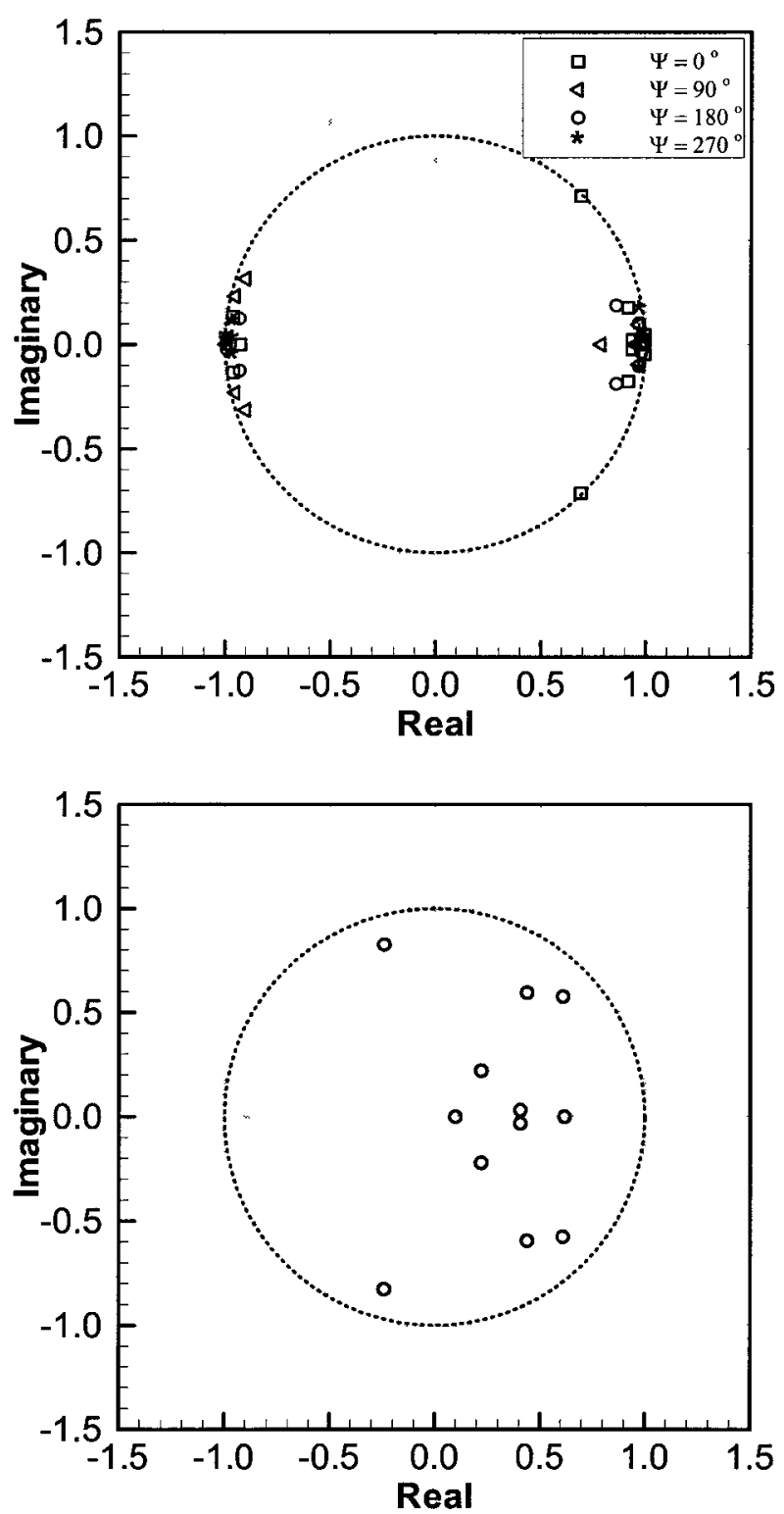

Figure 4.13: Distribution of the eigenvalues at azimuth Angles $\Psi=0,90,180,270$ degrees and the characteristic multipliers of the system.

\subsubsection{Validation Simulations}

The accuracy of the system model to predict the aeroelastic response obtained from the GENUVP/GAST computer simulations was validated for different TEF deflection scenar- 
ios. First, the LTP system model was compared with a LTI system model for a complete frequency range of TEF deflection $(0-5 / r e v)$. The LTI system model was obtained using the same ensemble data that was used to obtain the LTP system model. The physically realizable TEF deflections were applied only to the TEF of one blade during ten rotor revolutions (see Fig. 4.14). The responses obtained from the LTP and LTI system models are compared with the GENUVP/GAST aeroelastic computer simulations in Fig. 4.14.
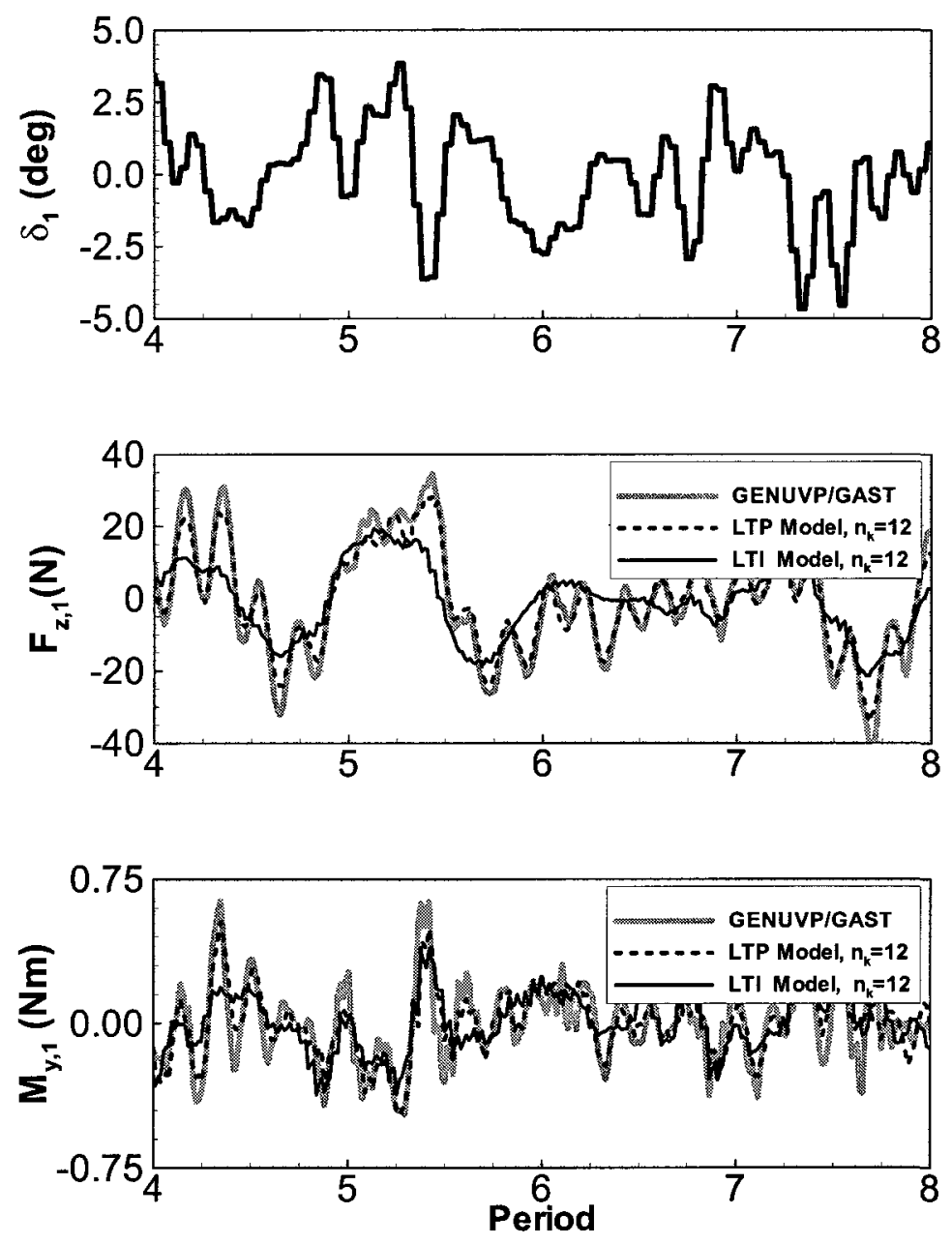

Figure 4.14: Comparison of LTP system assumption and LTI system assumption: blade loads, $F_{z, 1}$ and $M_{y, 1}$ to a physically realizable random TEF deflection obtained from LTP and LTI reduced-order models, and the GENUVP/GAST simulations. 
The relative percentage error norm $L_{2}$ between the LTI and LTP models and the GENUVP/GAST aeroelastic simulations for the defined random TEF deflections over ten revolutions are presented in Table 4.2 .

Table 4.2: The relative percentage error norm $L_{2}$ between the blade loads obtained from LTI and LTP models $\left(n_{k}=12\right)$ and the GENUVP/GAST aeroelastic simulations under the physically realizable random TEF deflection of one blade.

\begin{tabular}{|c|c|c|}
\hline System Model & LTI, $n=12$ & LTP, $n_{k}=12$ \\
\hline$\underline{F}_{z, 1}$ & 70.4 & 27.5 \\
\hline$\underline{M}_{y, 1}$ & 79.3 & 51.2 \\
\hline
\end{tabular}

Therefore, the LTI system having the same order fails to model the time-periodic helicopter rotor system's aeroelastic response. Greater modeling accuracy was obtained for $\underline{F}_{z, 1}$ by modeling the system as a LTP system as the percentage error decreased to $27 \%$.

For further analysis, the TEF of one blade was deflected at the critical frequencies $\left(\omega_{f}=1 /\right.$ rev, $3 /$ rev and $5 /$ rev $)$ for vibration suppression of the rotor systems. The helicopter rotor system's aeroelastic responses caused by the TEF of one blade at frequencies $\omega_{f}=1 /$ rev, $3 /$ rev and 5/rev are compared in Fig. 4.15 both in time and frequency domains. 

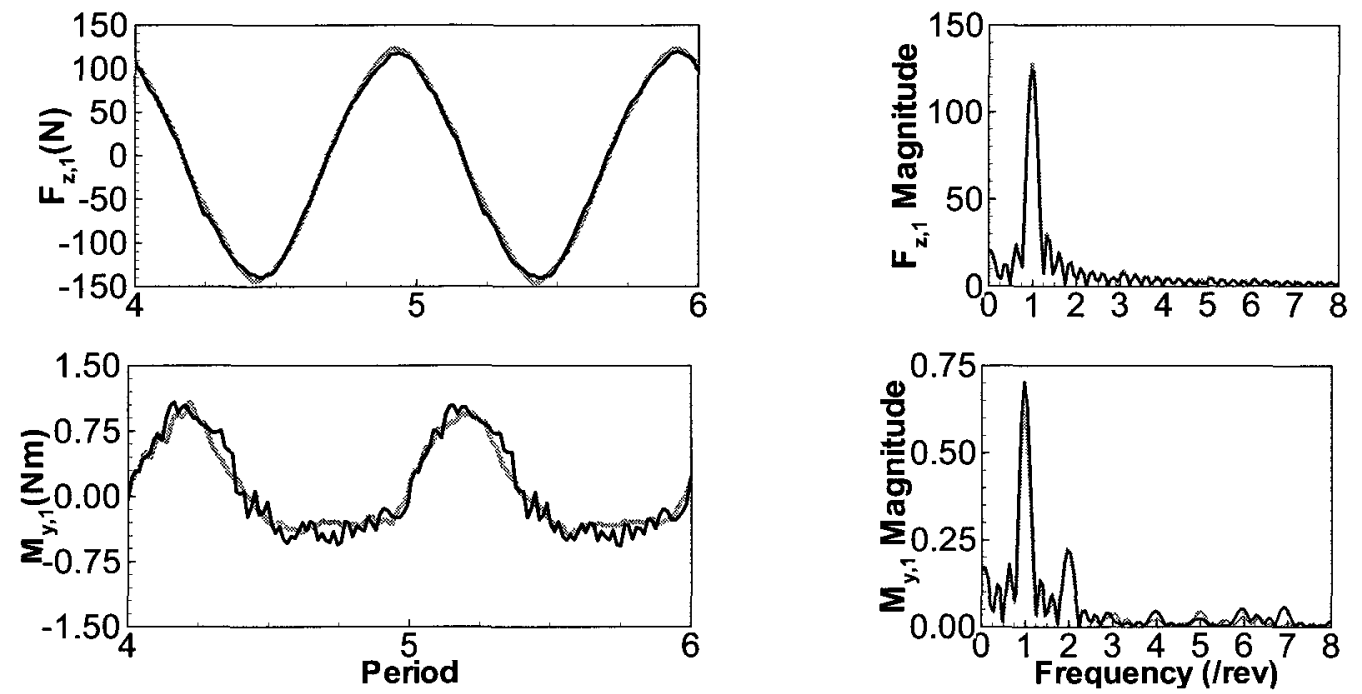

(a) TEF Deflection at $1 /$ rev
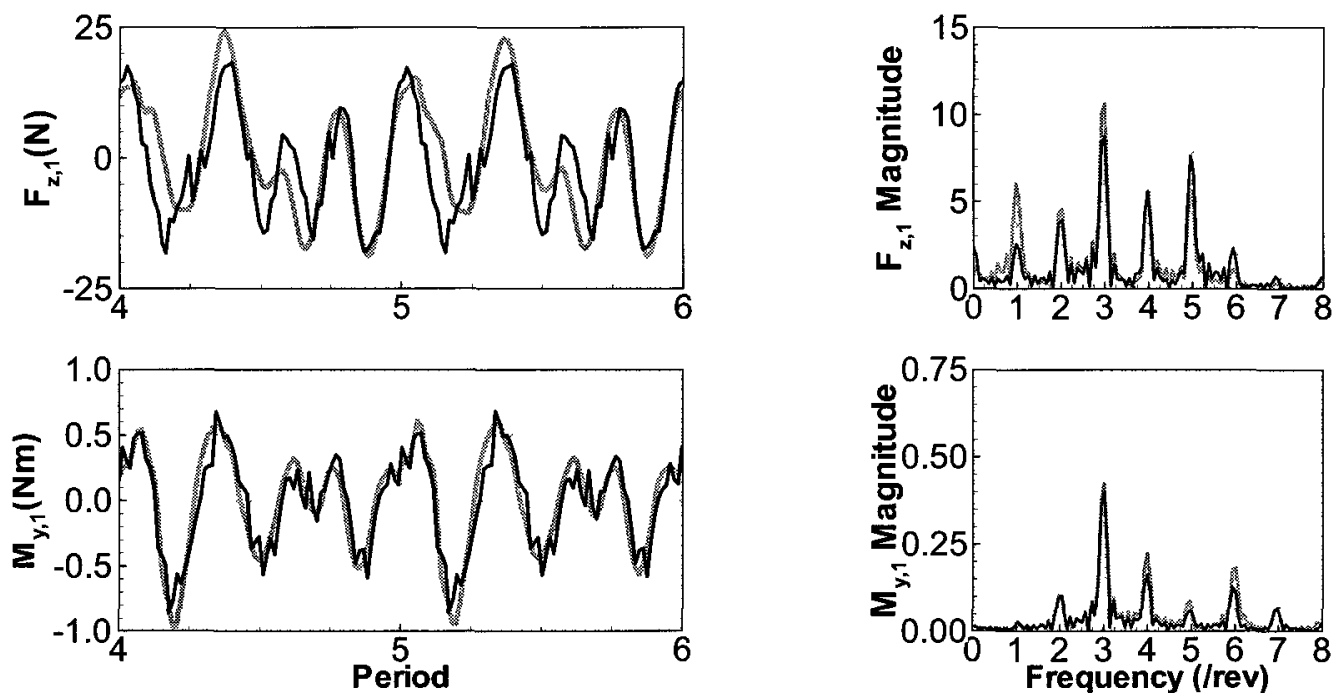

(b) TEF Deflection at $3 / r e v$

Figure 4.15: Comparison of blade loads $F_{z, 1}$ and $M_{y, 1}$ obtained from LTP system model and the GENUVP/GAST aeroelastic simulations when the TEF of one blade was deflected at a frequency of $1 /$ rev, $3 /$ rev and $5 /$ rev, respectively. 

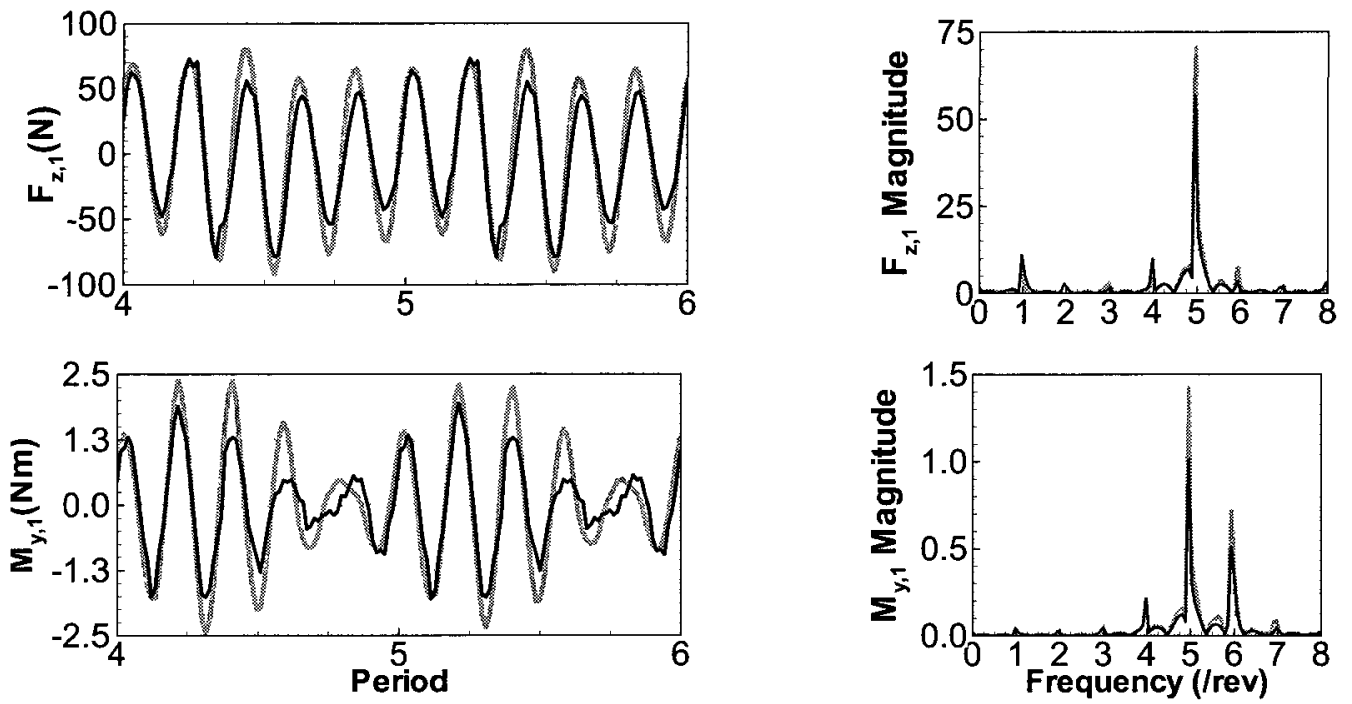

(c) TEF Deflection at $5 /$ rev

\section{GENUVP/GAST}

LTP Model, $n_{k}=12$

Figure 4.15: Comparison of blade loads $F_{z, 1}$ and $M_{y, 1}$ obtained from LTP system model and the GENUVP/GAST aeroelastic simulations when the TEF of one blade was deflected at a frequency of $1 / r e v, 3 / r e v$ and $5 / r e v$ respectively, (Cnt.)

The relative percentage error norm $L_{2}$ between the aeroelastic responses obtained from the system model and the GENUVP/GAST simulations, and the distribution of this error at frequencies $1 /$ rev, $\cdots, 6 /$ rev when the TEF of one blade was deflected at frequencies $1 /$ rev, $3 /$ rev and $5 /$ rev are presented in Tables 4.3 and 4.4, respectively. 
Table 4.3: Relative percentage error norm $L_{2}$ between the blade loads obtained from the LTP model of the One Blade Analysis with order $n_{k}=12$ and the GENUVP/GAST simulations when the TEF of one blade was deflected at the frequencies $1 /$ rev, $3 /$ rev and $5 /$ rev separately.

\begin{tabular}{|l|l|l|l|l|}
\hline \multirow{2}{*}{$\begin{array}{l}\text { Excitation } \\
\text { Frequency }\end{array}$} & \multicolumn{2}{|c|}{ Total Error } & \multicolumn{2}{c|}{$\begin{array}{l}\text { Error in Frequency } \\
\text { Range 0 - }\end{array}$} \\
\cline { 2 - 5 } & $\underline{F}_{z, 1}$ & $\underline{M}_{y, 1}$ & $\underline{F}_{z, 1}$ & $\underline{M}_{y, 1}$ \\
\hline $1 /$ rev & 5.7 & 29.8 & 3.4 & 14.3 \\
\hline $3 /$ rev & 57.6 & 37.8 & 31.3 & 19.1 \\
\hline $5 /$ rev & 30.0 & 39.5 & 23.7 & 29.0 \\
\hline
\end{tabular}

Table 4.4: Relative percentage error in amplitudes between the aeroelastic responses obtained from the system model and the GENUVP/GAST simulations at frequencies $1 /$ rev, $\cdots, 6 / r e v$, under the TEF excitation at frequencies $1 /$ rev, $3 /$ rev and $5 /$ rev.

\begin{tabular}{|c|c|c|c|c|c|c|c|c|c|c|c|c|}
\hline Excitation & \multicolumn{12}{|c|}{ Relative Percentage Error in Amplitude at Frequencies } \\
\hline \multirow[b]{3}{*}{$1 /$ rev } & \multicolumn{2}{|c|}{$1 /$ rev } & \multicolumn{2}{|c|}{$2 / r e v$} & \multicolumn{2}{|c|}{$3 / r e v$} & \multicolumn{2}{|c|}{$4 / r e v$} & \multicolumn{2}{|c|}{$5 / r e v$} & \multicolumn{2}{|c|}{$6 / r e v$} \\
\hline & $\underline{F}_{z, 1}$ & $\underline{M}_{y, 1}$ & $\underline{F}_{z, 1}$ & $\underline{M}_{y, 1}$ & $\underline{F}_{z, 1}$ & $\underline{M}_{y, 1}$ & $\underline{F}_{z, 1}$ & $\underline{M}_{y, 1}$ & $\underline{F}_{z, 1}$ & $\underline{M}_{y, 1}$ & $\underline{F}_{z, 1}$ & $\underline{M}_{y, 1}$ \\
\hline & 2.3 & 13.6 & 12.0 & 2.5 & - & - & - & 51.5 & - & 83.8 & $\underline{ }$ & 46 \\
\hline $3 / r e v$ & 57.6 & 17.0 & 13.3 & 0.8 & 6.0 & 17.7 & 36.8 & 0.7 & 44.2 & 32.0 & NA & 44.0 \\
\hline $5 /$ rev & 56.2 & - & - & - & - & - & NA & 1.4 & 19.9 & 40.8 & 51.4 & 38.8 \\
\hline
\end{tabular}

The tabulated results show that the aeroelastic responses are well captured at those frequencies at which the TEF of the one blade was excited, i.e. the contribution of the zeroth harmonic to the overall response. Moreover, the frequency responses at $\omega_{f} \pm 1 \omega_{p}$, $\omega_{p}=1 /$ rev, i.e. the contribution of the first harmonic, were also very well captured. However, at the excitation frequencies $3 / r e v$ and $5 / r e v$, the model was not capable of modeling the $1 /$ rev characteristics of the helicopter rotor system. Further analysis showed that the 
rotating frame blade load, $F_{z}$ resulted from the TEFs deflections with the $3 /$ rev, $4 /$ rev or $5 / r e v$ frequencies, shows nonlinearities at the $1 /$ rev frequency. The model can only capture the linear response, which explains the high prediction errors at $1 /$ rev frequency. Further investigations are necessary for the source of this nonlinearity, and left as future work.

\subsection{Discrete-Time Time-Periodic State-Space Realization us- ing Subspace Identification Part II: All Blade Analysis}

The all blade analysis includes the interaction of the blades through the disturbed wake vortices behind the TEFs in the discrete-time LTP state-space model realization. The results presented in Section 4.5 showed that the one blade analysis was able to predict the helicopter rotor system's aeroelastic response obtained from the GENUVP/GAST simulations when only the TEF of one blade was deflected. However, ignoring the interactions of the blades when the TEFs of all blades were deflected leads to errors in the analysis. These errors become significant at some specific frequencies, such as $3 /$ rev frequency that is primarily used for control purposes. In the all blade analysis, the model provides the map between the TEF deflection of one blade $\delta_{1_{k}}$ and the rotating frame blade loads of all blades $\underline{F}_{z, 1,2,3,4_{k}}$ and $\underline{M}_{y, 1,2,3,4_{k}} \forall k=1, \cdots, 24$. The same ensemble data set used and the order of the model was determined by following the same procedure outlined in Section 4.5. Since the model includes the dynamics of all blades, the order of the system was necessarily increased. The augmented state-space representation was used to have four-bladed rotor system analysis (see Eq. 4.6). The outputs of the augmented system were the rotating frame blade loads caused by the deflection of all the TEFs. To obtain the hub loads, a transformation in the coordinates was applied to the rotating frame blade loads according to each blade's azimuth location.

In Eq. 4.6, $y_{k_{j, i_{n}}}$ refers to the rotating frame blade loads of blades $j=1, \cdots, N_{b}$ caused by the $i_{n}{ }^{\text {th }}$ blade's TEF deflection; $j, i_{j}$ refers to the blade whose TEF was deflected, and $j=4$ refers to the blade following the blade whose TEF was deflected. The total blade load of blade number $j$ in the rotating frame, and the total vertical hub load caused only 
by the TEF deflection, $F_{Z}$, are given in Eq. 4.4 and Eq. 4.5 , respectively:

$$
\begin{aligned}
& F_{z_{\jmath}}=F_{z_{\jmath, 1}}+F_{z_{\jmath, 2}}+F_{z_{\jmath, 3}}+F_{z_{\jmath, 4}} \\
& F_{Z}=\sum_{j=1}^{4} F_{z_{\jmath}}
\end{aligned}
$$

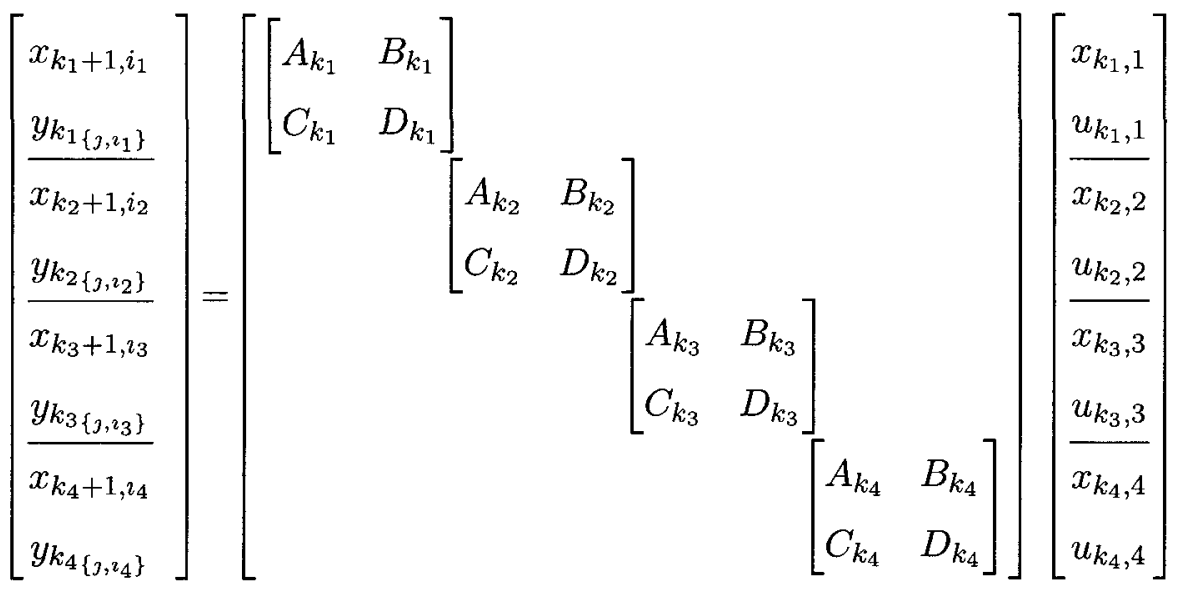

where $k_{i}=k_{1}+i_{n} N / N_{b}$ and $k_{1}=1, \cdots N / N_{b}$.

\subsubsection{Stability of the System}

The distribution of the eigenvalues of the system at different azimuth angles and the characteristic multipliers (eigenvalues of the monodromy matrix, $\Psi_{k}$ ) are presented in Fig. 4.16 for the augmented system of order $n_{k}=48, \forall k=1, \cdots, 24$. 

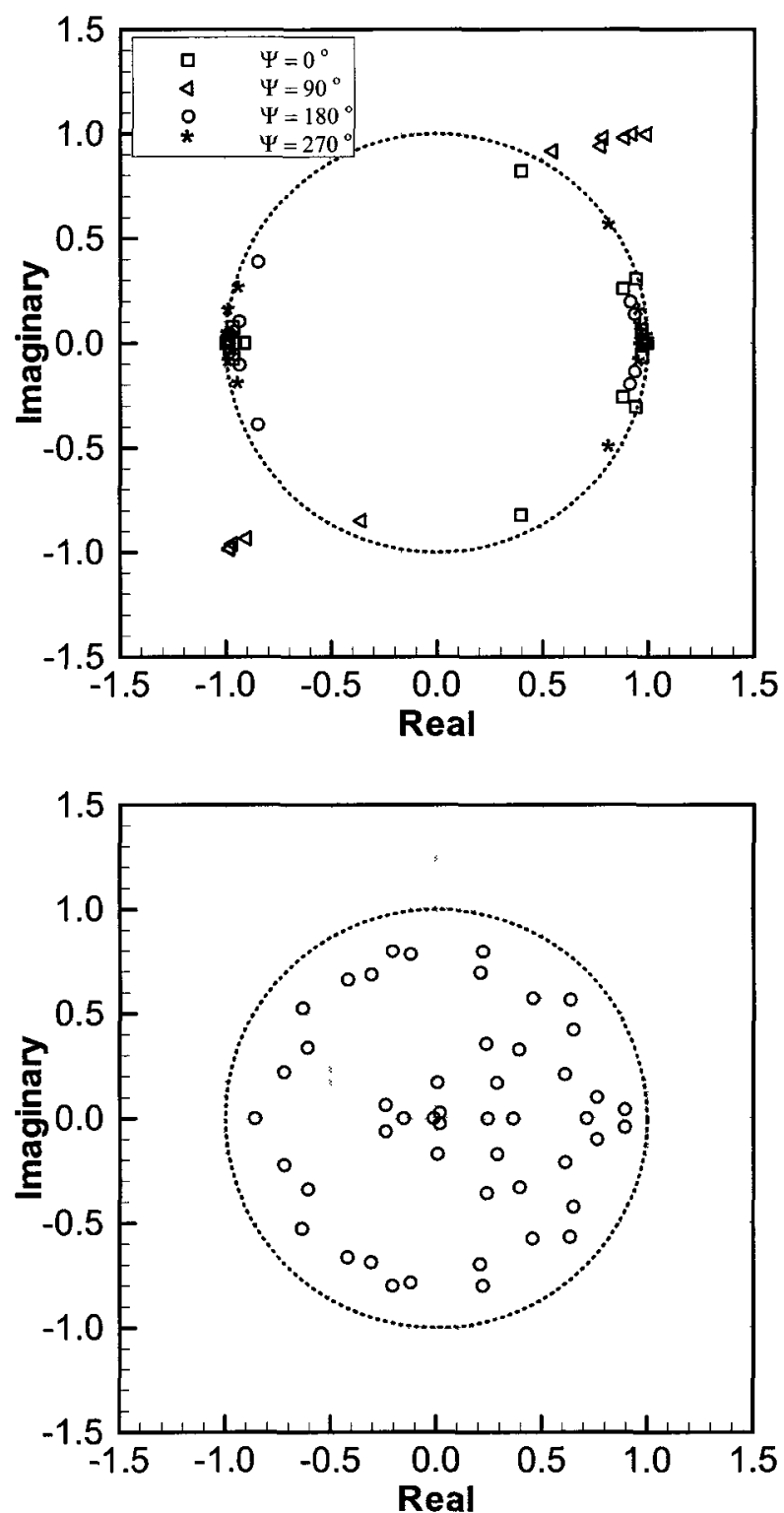

Figure 4.16: Distribution of the eigenvalues at azimuth angles, $\Psi=0,90,180,270$ degrees and the characteristic multipliers of the system.

Although, at azimuth angle $\Psi=90^{\circ}$, the system presents unstable poles, the characteristic multipliers lie in a unit circle. Therefore, the system is stable. The eigenvalues near the origin show the time delay in the response of the blades due to the deflection of the TEFs belonging to other blades. 


\subsubsection{Validation Simulations}

Validation simulations were carried out to compare the capabilities of the models obtained from the one blade and all blade analyses in predicting the aeroelastic response of helicopter rotor system for different TEF deflection scenarios. These validation results are summarized in this section.

The TEFs of all blades were deflected at $1 /$ rev frequency with a $90^{\circ}$ phase $\left(\delta_{i}=6 \sin \left(\Psi_{i}\right)\right.$ degrees). For this particular TEF deflection scenario, the rotating frame blade loads ( $\underline{F}_{z, 1,2,3,4_{k}}$ and $\left.\underline{M}_{y, 1,2,3,4}\right)$ and the hub loads $\left(F_{Z}, M_{X}\right.$ and $M_{Y}$ ) obtained from the GENUVP/GAST aeroelastic simulations and from the model simulations of the one blade analysis (1BA) and the all blades analysis (ABA) were compared in Fig. 4.17 and Fig. 4.18, respectively. The relative percentage error norm $L_{2}$ and the relative percentage error in amplitudes in the frequency range $0-6 /$ rev are presented in Table 4.5, Table 4.6 and Table 4.7 for the rotating frame blade loads and the hub loads, respectively. 

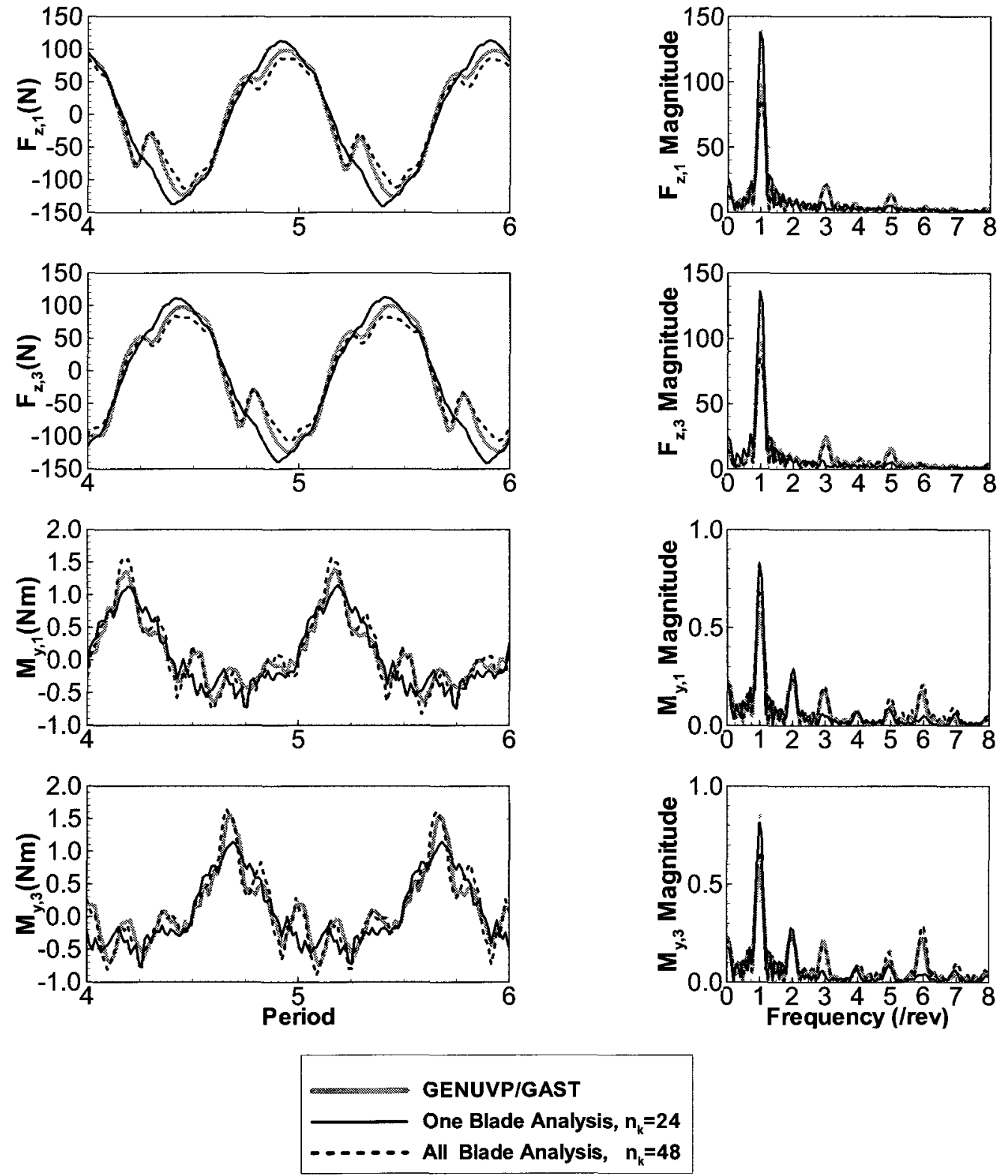

Figure 4.17: Comparison of the blade loads obtained from the LTP models of the one blade analysis (1BA) with order $n_{k}=24$, and the all blades analysis (ABA) with order $n_{k}=48$, and the GENUVP/GAST aeroelastic simulations when the TEF of all blades was deflected with a phase shift $\phi_{2}=90^{\circ}$ at $1 /$ rev frequency. 

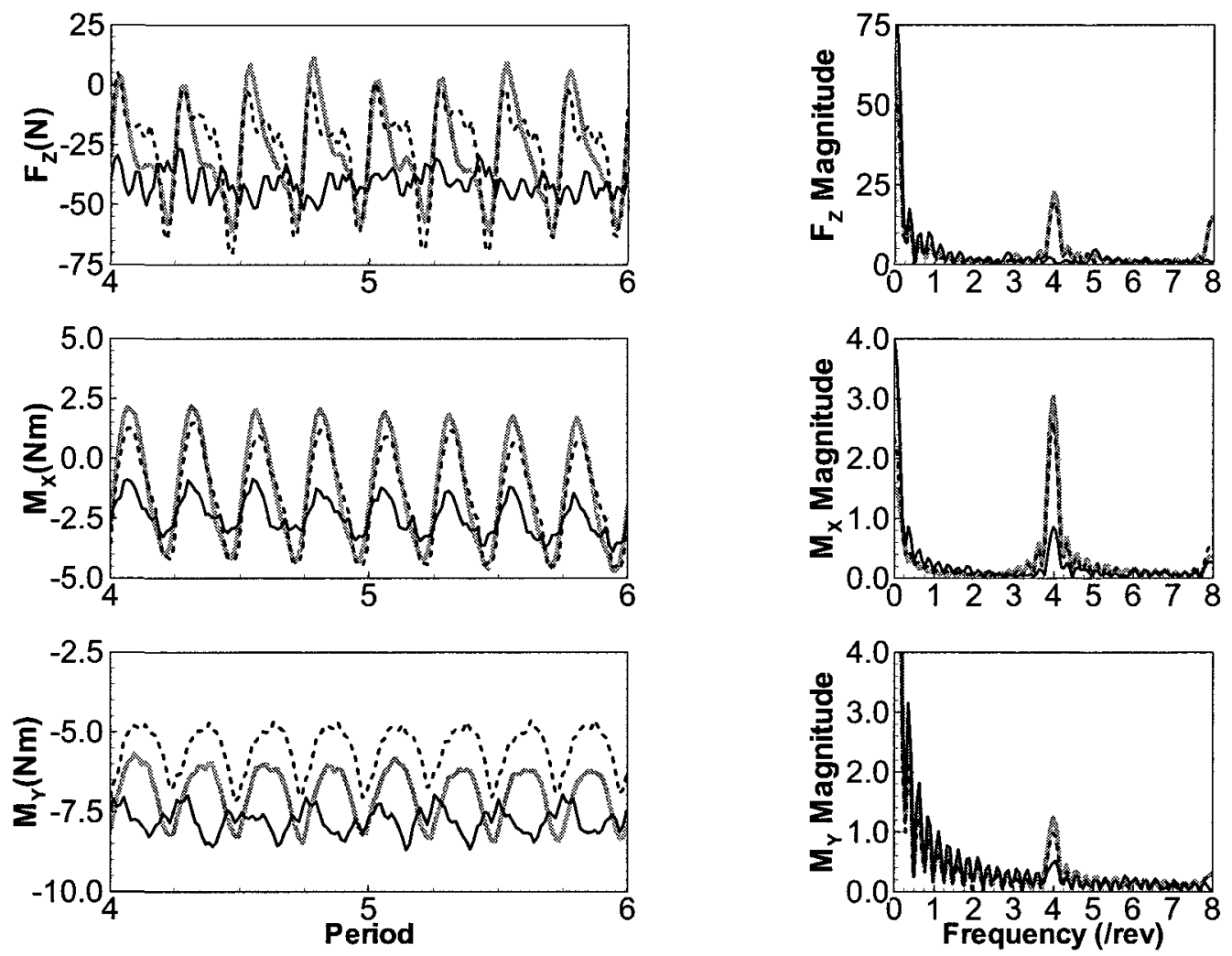

\section{GENUVP/GAST}

One Blade Analysis, $\mathrm{n}_{\mathrm{k}}=\mathbf{2 4}$

All Blade Analysis, $n_{k}=48$

Figure 4.18: Comparison of the hub loads obtained from the LTP models of the one blade analysis (1BA) with order $n_{k}=24$, and the all blade analysis (ABA) with order $n_{k}=48$, and the GENUVP/GAST aeroelastic simulations when the TEF of all blades was deflected with a phase shift $\phi_{\imath}=90^{\circ}$ at $1 /$ rev frequency. 
Table 4.5: The Relative percentage error norm $L_{2}$ between the blade loads obtained from the LTP models of the one blade analysis (1BA) with order $n_{k}=24$, and the all blades analysis (ABA) with order $n_{k}=48$, and the GENUVP/GAST aeroelastic simulations, when the TEF of all blades was deflected with a $\phi_{i}=90^{\circ}$ phase shift at $1 /$ rev frequency.

\begin{tabular}{|l|l|l|l|l|l|l|l|l|l|}
\hline \multicolumn{2}{|c|}{$\underline{F}_{z, 1}, \underline{F}_{z, 3}$} & \multicolumn{2}{c|}{$\underline{M}_{y, 1}, \underline{M}_{y, 3}$} & \multicolumn{2}{c|}{$\underline{F}_{Z}$} & \multicolumn{2}{c|}{$\underline{M}_{X}$} & \multicolumn{2}{c|}{$\underline{M}_{Y} \dagger$} \\
\hline $1 \mathrm{BA}$ & $\mathrm{ABA}$ & $1 \mathrm{BA}$ & $\mathrm{ABA}$ & $1 \mathrm{BA}$ & $\mathrm{ABA}$ & $1 \mathrm{BA}$ & $\mathrm{ABA}$ & $1 \mathrm{BA}$ & $\mathrm{ABA}$ \\
\hline $29.9,32.7$ & $13.7,14.9$ & $44.8,49.6$ & $31.5,28.9$ & 67.2 & 29.2 & 87.2 & 32.6 & 132.7 & 32.9 \\
\hline
\end{tabular}

†The mean values were subtracted.

Table 4.6: The relative percentage error in amplitudes between the blade loads obtained from the LTP models of the one blade analysis (1BA) with order $n_{k}=24$, and the all blades analysis (ABA) with order $n_{k}=48$, and the GENUVP/GAST simulations at the frequencies $1 /$ rev, $\cdots, 6 / r e v$, when the TEFs of all blades were deflected with a $\phi_{i}=90^{\circ}$ phase shift at $1 /$ rev frequency.

\begin{tabular}{|c|c|c|c|c|c|c|c|c|}
\hline Frequency & \multicolumn{2}{|c|}{$\underline{F}_{z, 1}$} & \multicolumn{2}{|c|}{$\underline{F}_{z, 3}$} & \multicolumn{2}{|c|}{$\underline{M}_{y, 1}$} & \multicolumn{2}{|c|}{$\underline{M}_{y, 3}$} \\
\hline & $1 \mathrm{BA}$ & $\mathrm{ABA}$ & $1 \mathrm{BA}$ & $\mathrm{ABA}$ & $1 B A$ & ABA & $1 \mathrm{BA}$ & $\mathrm{ABA}$ \\
\hline $1 /$ rev & 42.7 & 11.0 & 41.4 & 12.8 & 44.2 & 17.9 & 40.9 & 15.1 \\
\hline $2 / r e v$ & 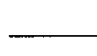 & 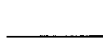 & 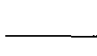 & 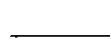 & 25.3 & 3.7 & 15.7 & 1.7 \\
\hline $3 /$ rev & 65.8 & 1.6 & 74.3 & 18.7 & 67.5 & 10.4 & 72.0 & 10.7 \\
\hline $4 / r e v$ & 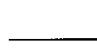 & 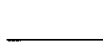 & 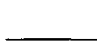 & 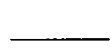 & 5.9 & 11.1 & 10.4 & 14.3 \\
\hline $5 /$ rev & 61.9 & 7.5 & 68.6 & 19.9 & 13.2 & 41.7 & 23.2 & 41.6 \\
\hline $6 /$ rev & 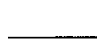 & 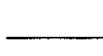 & 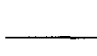 & 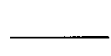 & 72.9 & 20.8 & 82.7 & 27.3 \\
\hline
\end{tabular}


Table 4.7: The relative percentage error in amplitudes between the hub loads obtained from the LTP models of the one blade analysis (1BA) with order $n_{k}=24$, and the all blade analysis (ABA) with order $n_{k}=48$, and the GENUVP/GAST simulations at the frequencies $1 / \mathrm{rev}, \cdots, 6 / \mathrm{rev}$, when the TEFs of all blades were deflected with a $\phi_{\imath}=90^{\circ}$ phase shift at $1 /$ rev frequency.

\begin{tabular}{|l|l|l|l|l|l|l|}
\hline \multirow{2}{*}{ Frequency } & \multicolumn{2}{|c|}{$F_{Z}$} & \multicolumn{2}{c|}{$M_{X}$} & \multicolumn{2}{c|}{$M_{Y}$} \\
\cline { 2 - 7 } $0 /$ rev & $1 \mathrm{BA}$ & $\mathrm{ABA}$ & $1 \mathrm{BA}$ & $\mathrm{ABA}$ & $1 \mathrm{BA}$ & $\mathrm{ABA}$ \\
\cline { 2 - 7 } & 40.0 & 6.5 & 70.0 & 11.2 & 10.8 & 21.8 \\
\hline $4 /$ rev & 89.2 & 12.0 & 71.6 & 13.5 & 57.8 & 20.0 \\
\hline $8 /$ rev & $\mathrm{NA}$ & 1.4 & 24.8 & 48.2 & $\mathrm{NA}$ & 12.0 \\
\hline
\end{tabular}

When all the TEFs were deflected at $1 /$ rev frequency with a $90^{\circ}$ phase shift, the blade loads $\underline{F}_{z, 1,2,3,4}$ and $\underline{M}_{y, 1,2,3,4}$ experience additional $3 /$ rev, 4rev, 5/rev and $3 /$ rev, $4 / r e v, 5 / r e v$, and $6 / r e v$ frequency components, respectively. The increase in $3 /$ rev frequency component is the greatest for both $F_{z}$ and $M_{y}$, in addition the increase in the $6 / r e v$ frequency component of $M_{y}$ is not negligible. The increase in these frequency components of the blade loads was not observed when the TEF of one blade was deflected and the blade loads on the same blade was analyzed. Since all $3 / r e v, 4 / r e v$, and $5 / r e v$ frequency components of blade loads in rotating frame contributes directly to the hub loads, the resultant hub loads can only be predicted accurately if the interaction of the blades through the disturbed wake is taken into consideration. This is a very important observation regarding control design.

The trim conditions were altered by imposing $1 /$ rev deflections to TEFs. This requires a simultaneous control of swashplate angles and the TEF deflections to satisfy the trim conditions in case of TEF deflections at 1/rev frequency. In the frame work of this thesis, the swashplate settings were kept constant at the baseline trim state condition regardless of the TEF deflections imposed.

The ability of the model to capture the aeroelastic response of the helicopter rotor system was analyzed in the case of aerodynamic dissimilarity in the blade system. Phase-shifted 
TEF deflections were imposed to all blades at 3/rev frequency as shown in Fig. 4.19. The helicopter rotor system's aeroelastic responses, in terms of blade loads $\left(\underline{F}_{z, 1,2,3,4}, \underline{M}_{y, 1,2,3,4}\right)$ and the hub loads $\left(\underline{F}_{Z}, \underline{M}_{X}, \underline{M}_{Y}\right)$ obtained from the GENUVP/GAST aeroelastic code simulations and from the models, are compared in Fig. 4.20 and Fig. 4.21.

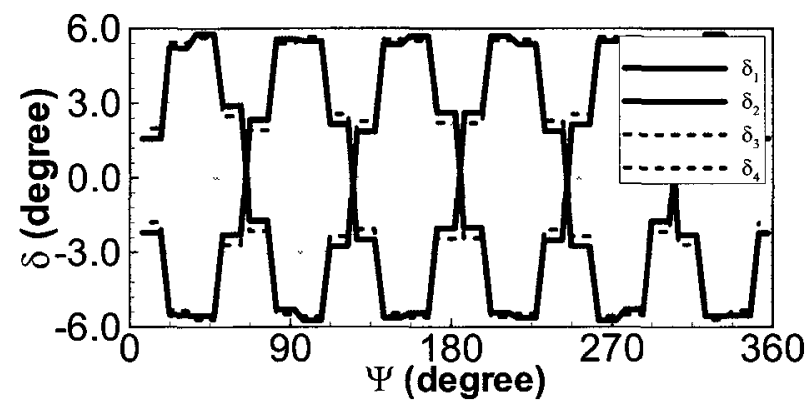

Figure 4.19: Phase-shifted TEF deflections at $3 /$ rev frequency $\left(\delta_{i}=6 \sin \left(3 \Omega+\phi_{i}\right)\right.$ degrees. $)$ 

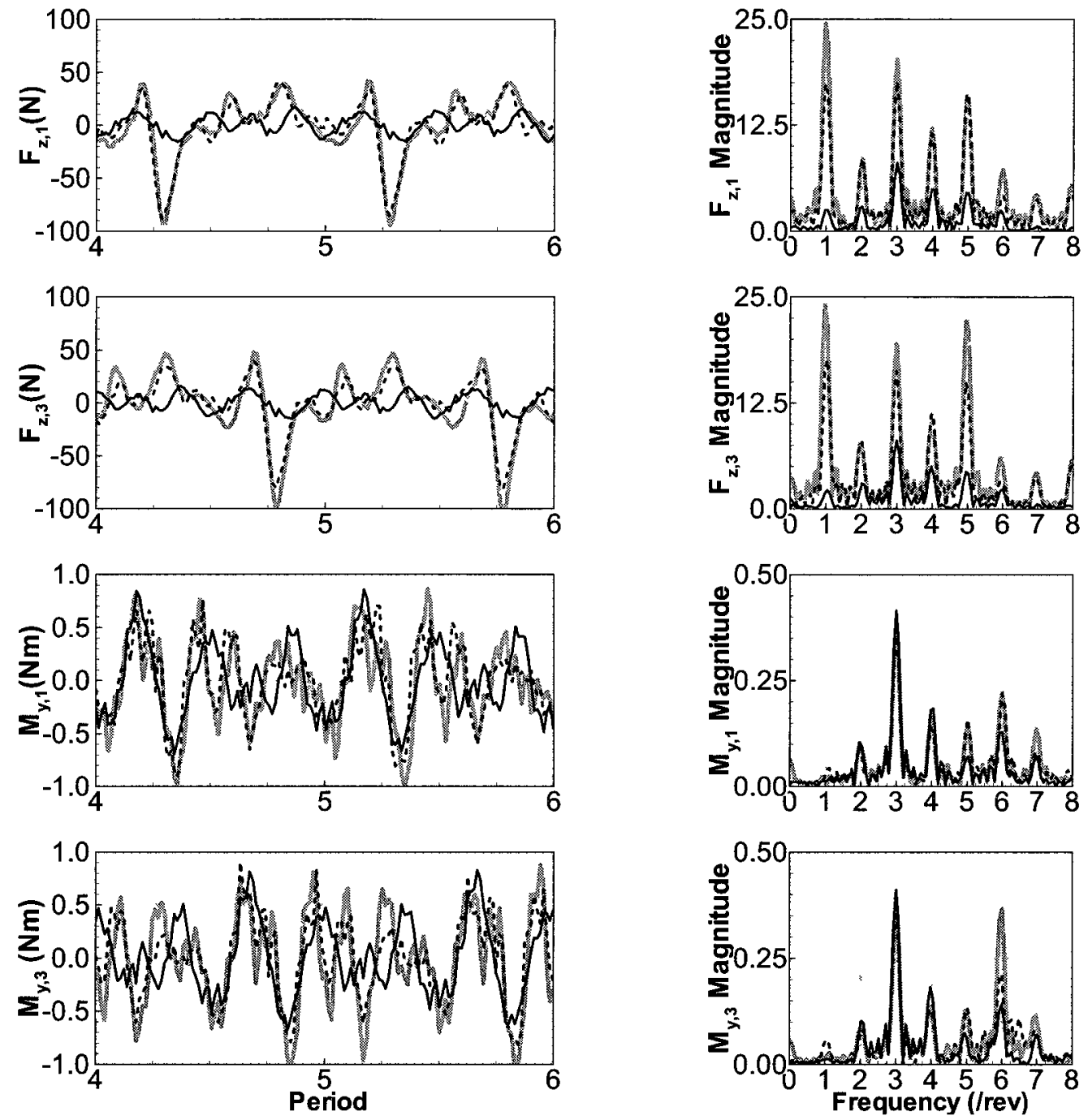

GENUVP/GAST

One Blade Analysis, $n_{k}=24$

All Blade Analysis, $n_{k}=48$

Figure 4.20: Comparison of the blade loads obtained from the LTP models of the one blade analysis $(1 \mathrm{BA})$ with order $n_{k}=24$, and the all blade analysis $(\mathrm{ABA})$ with order $n_{k}=48$, and the GENUVP/GAST aeroelastic simulations when the TEF of all blades was deflected with a phase shift $\phi_{2}$ at $3 / r e v$ frequency. 

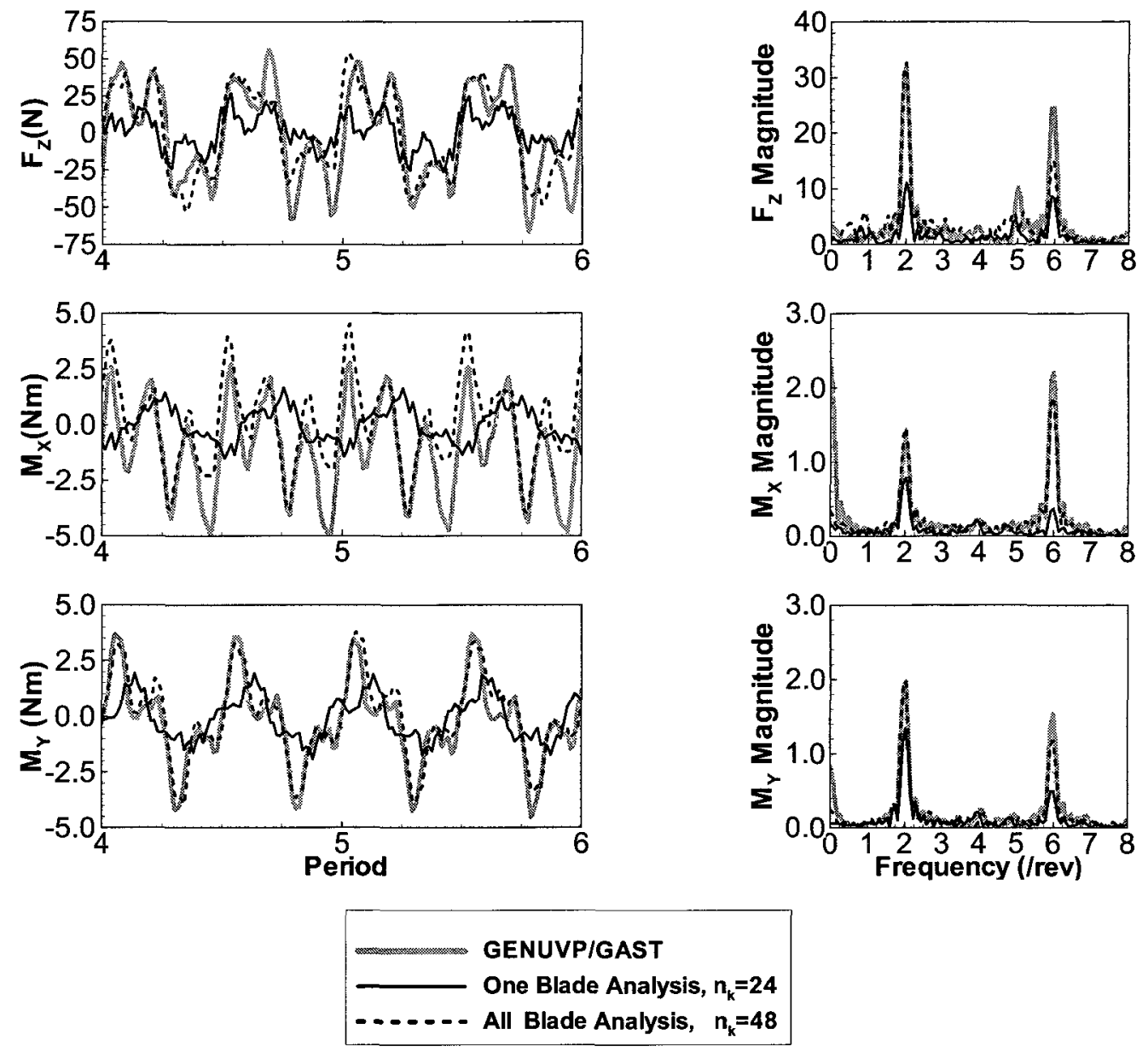

Figure 4.21: Comparison of the hub loads obtained from the LTP models of the one blade analysis (1BA) with order $n_{k}=24$, and the all blade analysis (ABA) with order $n_{k}=48$, and the GENUVP/GAST aeroelastic simulations when the TEF of all blades was deflected with a phase shift $\phi_{\imath}$ at $3 / r e v$ frequency.

Figure 4.21 shows that isolating the blade from the other blades, as in the case of the one blade analysis, indeed leads to inaccurate results, especially when the TEFs are deflected at $3 / r e v$ frequency. In this TEF deflection scenario, over $50 \%$ of the total vertical blade loads, $F_{z}$ recorded in each blade are caused by the TEF deflections of the other blades. Although, the one blade analysis was capable of predicting the effect of 3/rev deflections of one blade's TEF (see Fig. 4.15(b)), when all the blades were activated the one blade analysis did not 
predict the rotor aeroelastic response correctly. There is still some discrepancy in the prediction of the blade load $F_{z}$ at $1 /$ rev frequency in the all blade analysis. By increasing the system order, this error was decreased but could not be completely avoided. Since, $F_{z}$ resulted from the TEFs deflections with the $3 /$ rev frequency has nonlinearities at the $1 /$ rev frequency.

The blade loads of the blade number one and three, $\left(F_{z, 1,3}\right.$ and $\left.M_{y, 1,3}\right)$, that are obtained from the GENUVP/GAST aeroelastic code simulations were not equivalent at $5 /$ rev and $6 / r e v$ frequencies, respectively, at their steady-state values. One important reason for the higher error in the blade load prediction of the blade number three at these specific frequencies is the assumption of the blade equivalency (see Table 4.8).

The relative percentage $L_{2}$ norm of the error between responses obtained from the GENUVP/GAST aeroelastic simulations and from the discrete-time LTP state-space models are given for different system orders in Fig. 4.22. As the order of the system increased for the all blade analysis, the error decreases for the blade load $F_{z, 1}$. However, increasing the order did not change the accuracy of the $M_{y, 1}$ prediction. Even if the order of the system is increased in the one blade analysis, ignoring the blade interactions through the disturbed wake gives erroneous results in predicting the aeroelastic response of helicopter rotor system. 


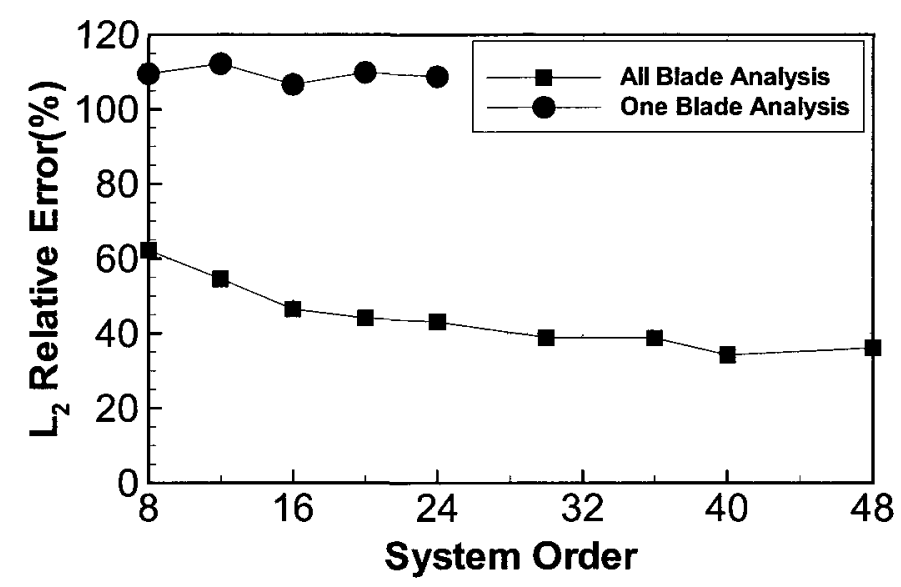

Figure 4.22: Relative percentage error norm $L_{2}$ between the blade loads $F_{z, 1}$ obtained from GENUVP/GAST simulation and identified model of different orders for the one blade analysis and the all blades analysis.

Table 4.8: Relative percentage error in amplitudes between the blade loads obtained from the LTP models of the one blade analysis (1BA) with order $n_{k}=24$, and all blade analysis (ABA) with order $n_{k}=48$, and the GENUVP/GAST simulations at the frequencies $1 /$ rev, $\cdots, 6 / r e v$, when the TEFs of all blades were deflected with a phase shift $\phi_{i}$ at $3 / r e v$ frequency.

\begin{tabular}{|l|l|l|l|l|l|l|l|l|}
\hline \multirow{2}{*}{ Frequency } & \multicolumn{2}{|c|}{$F_{z, 1}$} & \multicolumn{2}{c|}{$F_{z, 3}$} & \multicolumn{2}{c|}{$M_{y, 1}$} & \multicolumn{2}{c|}{$M_{y, 3}$} \\
\cline { 2 - 10 } $1 /$ rev & 1BA & ABA & $1 \mathrm{BA}$ & ABA & $1 \mathrm{BA}$ & ABA & $1 \mathrm{BA}$ & $\mathrm{ABA}$ \\
\cline { 2 - 10 } & 89.7 & 28.9 & 90.8 & 26.7 & & & & \\
\hline $2 /$ rev & 66.0 & 0.2 & 54.2 & 2.7 & 9.6 & 3.5 & 15.0 & 5.7 \\
\hline $3 /$ rev & 60.2 & 16.6 & 56.4 & 16.0 & 13.2 & 2.7 & 15.8 & 0.9 \\
\hline $4 /$ rev & 59.1 & 4.7 & 42.0 & 9.9 & 8.2 & 18.9 & 26.4 & 3.7 \\
\hline $5 /$ rev & 70.7 & 4.3 & 80.2 & 32.5 & 51.8 & 7.3 & 31.6 & 6.3 \\
\hline $6 /$ rev & 66.0 & 41.5 & 61.6 & 45.7 & 41.4 & 0.4 & 61.5 & 42.1 \\
\hline
\end{tabular}


Table 4.9: Relative percentage error in amplitudes between the hub loads obtained from the LTP models of the one blade analysis (1BA) with order $n_{k}=24$, and the all blade analysis (ABA) with order $n_{k}=48$, and the GENUVP/GAST simulations at the frequencies $1 /$ rev $, \cdots, 6 / r e v$, when the TEFs of all blades were deflected with a phase shift $\phi_{\imath}$ at $3 /$ rev frequency.

\begin{tabular}{|l|l|l|l|l|l|l|}
\hline \multirow{2}{*}{ Frequency } & \multicolumn{2}{|c|}{$F_{Z}$} & \multicolumn{2}{c|}{$M_{X}$} & \multicolumn{2}{c|}{$M_{Y}$} \\
\cline { 2 - 7 } & 1BA & $\mathrm{ABA}$ & $1 \mathrm{BA}$ & $\mathrm{ABA}$ & $1 \mathrm{BA}$ & $\mathrm{ABA}$ \\
\cline { 2 - 7 } $2 /$ rev & 65.2 & 2.2 & 40.6 & 9.7 & 32.2 & 0.2 \\
\hline $6 /$ rev & 64.4 & 39.0 & 83.3 & 14.9 & 67.5 & 23.2 \\
\hline
\end{tabular}

\subsection{Aerodynamic and Aeroelastic Analysis of Trailing Edge Flap Deflection}

The mapping identification between the TEF deflection and the rotating frame blade loads, and the resultant hub loads was carried out in the previous section. It was observed that if the interaction of the blades through the disturbed wake is ignored, both the blade and hub loads can not be modeled accurately. Specifically at TEF deflection at the $3 /$ rev frequency, the one blade analysis did not provide an accurate prediction of the rotating frame blade loads and hub loads at the frequencies of main interest. In this section, a detailed analysis on the effect of TEF on its own blade and on the other blades is presented with a pure aerodynamic analysis and modified aeroelastic coupling cases.

The GENUVP simulation results in terms of total aerodynamical lift and moment coefficients measured along the blade span were compared for different TEF deflections in Fig. 4.23 and Fig. 4.24. In all TEF deflection scenarios considered (named as one-blade, all blades, all blades-shift), the TEF of the blade one, $\delta_{1}$ experiences the same TEF deflection at $3 /$ rev with a zero phase shift. In the one-blade TEF deflection scenario, a zero TEF deflection was imposed on the other blades. In the all blades TEF deflection scenario, the TEF deflection was given as $\delta_{\imath}=6 \sin \left(3 \Psi_{\imath}\right)$ degrees, $i=1, \cdots, 4$, which depends on the blade's azimuth location. In the all blades shift TEF deflection scenario, the TEF deflection of blades follow each other with an opposite phase, as described in Fig. 4.19. 

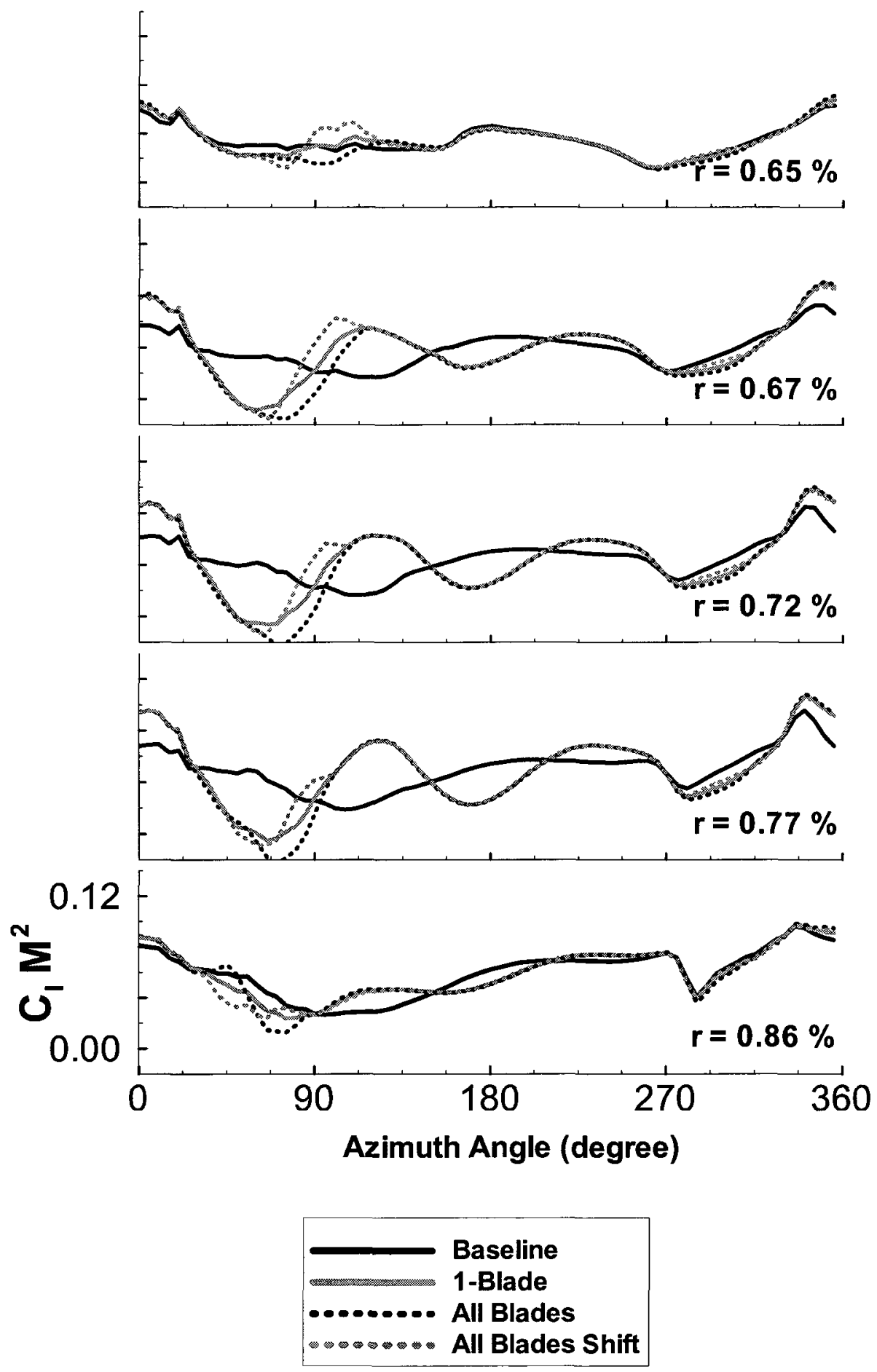

Figure 4.23: Sectional lift coefficient. 

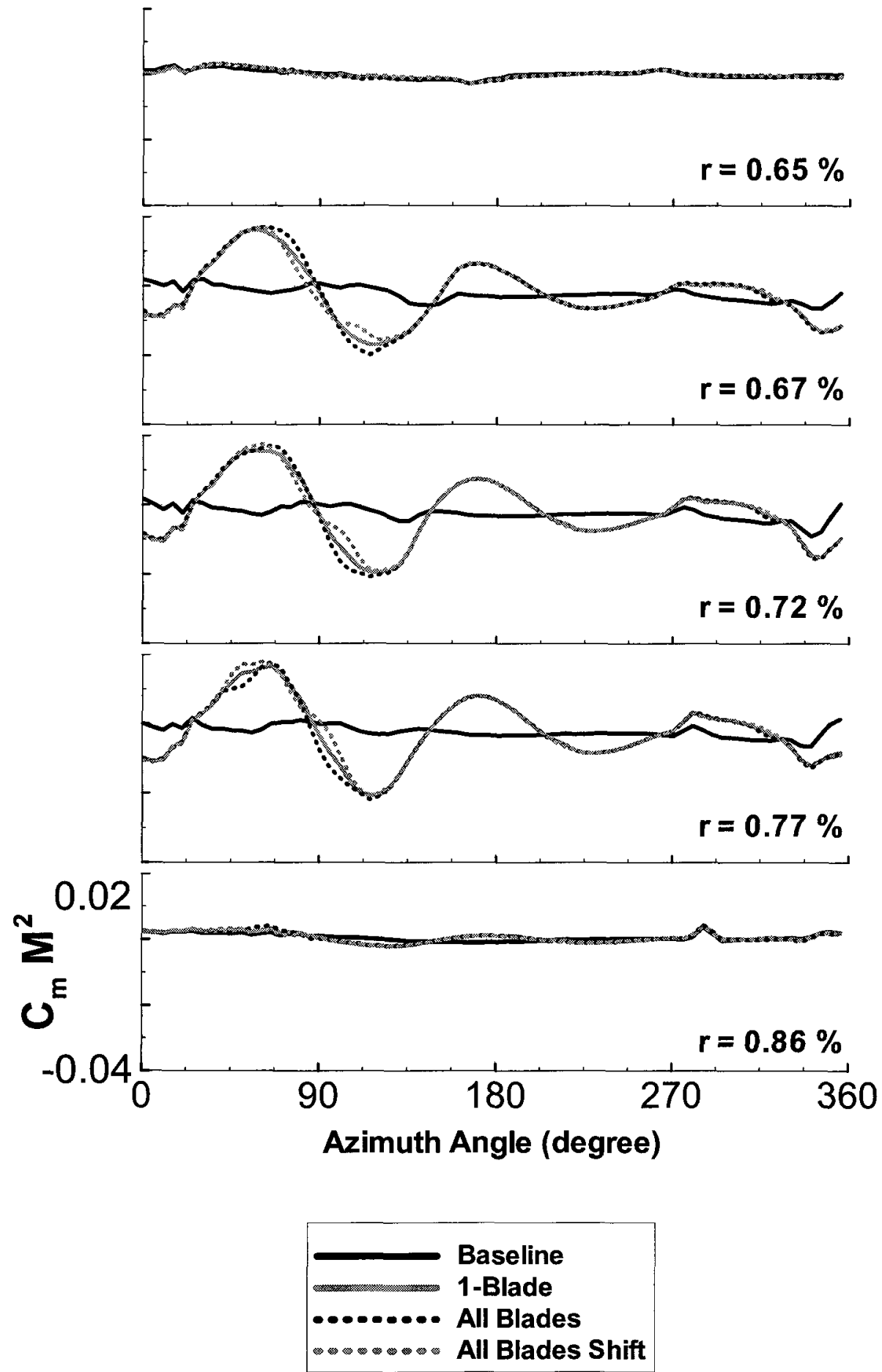

Figure 4.24: Sectional moment coefficient. 
The TEF deflection changes the lift and moment coefficients at the blade section where the TEF is attached. This change is greater around the $\% 77-\% 85$ spanwise location. This result was experimentally verified as well [130]. In addition, even though the $\delta_{1}$ TEF deflection is the same in all simulations, the aerodynamic lift and moment vary according to the preceding blade's TEF deflections. This variation is summarized as follows;

- The additional aerodynamic lift generated by the TEF deflection on the blade section is greater when all TEFs are activated rather than if only one TEF is activated. However, the amount of additional lift generated by the TEF deflection is also based on the spanwise location of the TEF as well as the azimuth location of the blade.

- The aerodynamic lift is influenced more then the sectional aerodynamic moment by the TEF deflection of the preceding blades.

- The greatest influence on the aerodynamic lift and moment was observed in the ranges of azimuth angles $\Psi=60^{\circ}-120^{\circ}$ and $\Psi=270^{\circ}-300^{\circ}$.

- In the azimuth angle range $\Psi=60^{\circ}-120^{\circ}$, the profile for the lift and moment change is strongly dependent on the type of the TEF deflection scenarios considered as listed above.

Therefore, based on pure aerodynamic analysis, it is concluded that the sectional aerodynamic loading changes depending on the TEF deflection of the preceding blades. While the influence of the TEF deflection on its own blade is the highest, the deflection of the TEF of the preceding blades is also significant specifically in the azimuth angle range $\Psi=60^{\circ}-120^{\circ}$. Since for the forward flight regime the wake vortices emitted from the preceding blade interacts with and/or impacts on the following blades in the azimuth angle range $\Psi=60^{\circ}-120^{\circ}$ (see Fig. 4.25), this observation is crucial for control design purpose. In addition, at the retreading side, depending on the shaft angle settings, the wake vortices would also interact with and/or impacts on the following blades in a similar manner. 


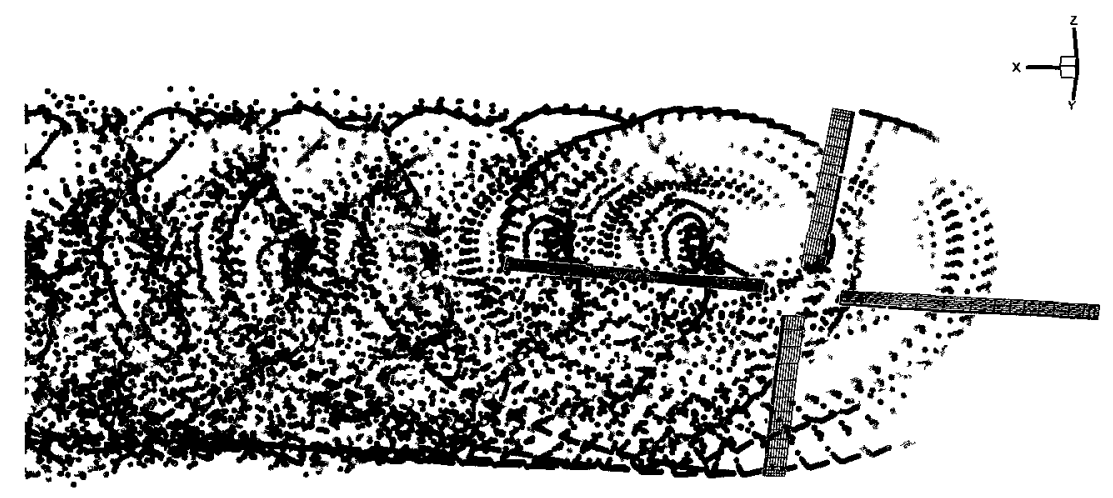

Figure 4.25: Wake of the four-bladed helicopter rotor system in forward flight.

The frequency content of the sectional aerodynamic lift and moment was analyzed at the blade sections having TEFs. For the defined 3/rev TEF deflections, the frequency content of aerodynamic lift coefficient at different spanwise location differs. When all the TEFs are deflected in the same way with respect to their azimuth angle (the all blades TEF deflection scenario) and with a specified phase shift (the all blades shift TEF deflection scenario), there are a greater $1 / r e v$ and $2 / r e v$ frequency components in the aerodynamic lift as compared to the one-blade TEF deflection case. This was verified for all spanwise locations analyzed. For all spanwise locations analyzed, the $3 /$ rev frequency component of the aerodynamic lift coefficient is higher when the TEFs are deflected in the same way with respect to their azimuth angle as compared to the one-blade TEF deflection case. In addition, the $4 / r e v$ component of the aerodynamic lift changes with respect to the TEF deflection and the spanwise location. The one blade and all blades analysis resulted in a decrease in the $4 / r e v$ component of aerodynamic lift with respect to the baseline case in all spanwise locations under consideration. But, the all blades shift analysis increased the 4/rev aerodynamic lift in the inner board $(65 \%-72 \%)$ and decreased in the outer board locations. This explains why the one blade analysis could not well predict the $4 /$ rev component of hub vertical load, $F_{Z}$. The $3 /$ rev TEF deflections change the sectional aerodynamic moment coefficients mostly at $2 / r e v, 3 / r e v, 4 / r e v$ frequencies. This change is the highest at $72 \%-77 \%$ spanwise locations. The difference between them is not considerable as compared to the difference in 
the aerodynamic lift coefficient. In conclusion, the analysis of aerodynamic loading on the blades shows that the sectional aerodynamic lift is highly influenced by the TEF deflection of the preceding blades. However, the sectional aerodynamic moment is mainly based on the TEF deflection of the same blade.

It was shown that when the TEFs of all blades were deflected with $1 /$ rev, the blade loads have 3/rev frequency content (see Fig. 4.17). This frequency content could not be observed if only the effect of TEF on its corresponding blade was analyzed and the TEFs of other blades were not deflected (see Fig. 4.17 and Fig. 4.15(a)). The question remains whether the $3 / r e v$ frequency content of the blade loads is caused by the interaction of wake vortices emanating from the preceding blades with the following blade, or by the specific structural property of the SHARCS's blade, i.e. this conclusion is valid only for the specific rotor analyzed. Since the first elastic flap bending mode of the SHARCS blade is very close to the $3 / r e v$ frequency, this property might cause a $3 / \mathrm{rev}$ frequency enhancement in the blade loads. To further investigate this additional frequency content at $3 / r e v$ frequency, a set of simulations were carried out using a softened blade in the flapwise bending direction, whose eigenfrequencies are given in the Table 4.10 .

Table 4.10: Comparison of eigen frequencies of the SHARCS blade and the softened blade at a rotational speed of $\Omega=162.8 \mathrm{rad} / \mathrm{s}$.

\begin{tabular}{lcc}
\hline Mode Number & \multicolumn{2}{c}{ Frequency $(/$ rev $)$} \\
& SHARCS Blade & Softened Blade \\
Rigid Lead-Lag & 0.24 & 0.24 \\
Rigid Flapwise Beam Bending & 1.03 & 1.03 \\
Elastic Flapwise Beam Bending & 2.96 & 2.80 \\
Elastic Chordwise Beam Bending & 5.23 & 4.88 \\
Coupled Elastic Flapwise Beam & 5.26 & 4.49 \\
Bending -Elastic Torsion & & \\
Elastic Torsion & 6.48 & 6.41 \\
\hline
\end{tabular}


Next, the TEFs of all blades were deflected at $1 /$ rev and $3 / r e v$ frequencies with a predefined phase shift. The helicopter rotor system's aeroelastic responses in terms of blade loads and hub loads are compared in Fig. 4.26 and Fig. 4.27 for the SHARCS blade and the softened blade.

Both the SHARCS blade and the softened blade experienced the blade loads having similar frequency content when the TEFs of all the blades are deflected. Softening the blade at the first elastic flap bending mode (from 2.96/rev to $2.80 /$ rev) decreased the $3 /$ rev excitation at the blade loads $F_{z, 1}$, when the TEF of all the blades were deflected with $1 /$ rev frequency with $90^{\circ}$ phase shift. However, there is still a $3 /$ rev frequency excitation at $F_{z, 1}$, which was not excited when only the TEF of one blade was deflected (see Fig. 4.15(a)). Although, there is a slight decrease in the $3 /$ rev excitation when the first flapping mode was shifted away from the integer multiple of rotational frequency, we cannot associate this additional $3 /$ rev excitation at $1 /$ rev TEF deflection of all blades by only having the particular structural property of the SHARCS blade. The same conclusion was drawn when the TEF of all blades were excited at $3 / r e v$ frequency with a phase shift (see Fig. 4.27). Knowing that the frequency content of the pure aerodynamic loading (without aeroelastic coupling) on the structure changes according to the TEF deflection of all blades, it is concluded that this additional excitation is mainly caused by the aerodynamics, i.e. the interaction of the blades with the wake vortices emanating from the preceding blades. In the previous research [14], there were some discrepancies between the comprehensive aeroelastic analysis and the experimental results performed with TEFs recorded and they were left as open questions. In [14], the comprehensive aeroelastic code utilized was based on a finite state approximation with a free-wake model that did not account for the TEF deflections. We have shown that the errors might been caused by the unmodelled disturbed wake behind the TEFs. 

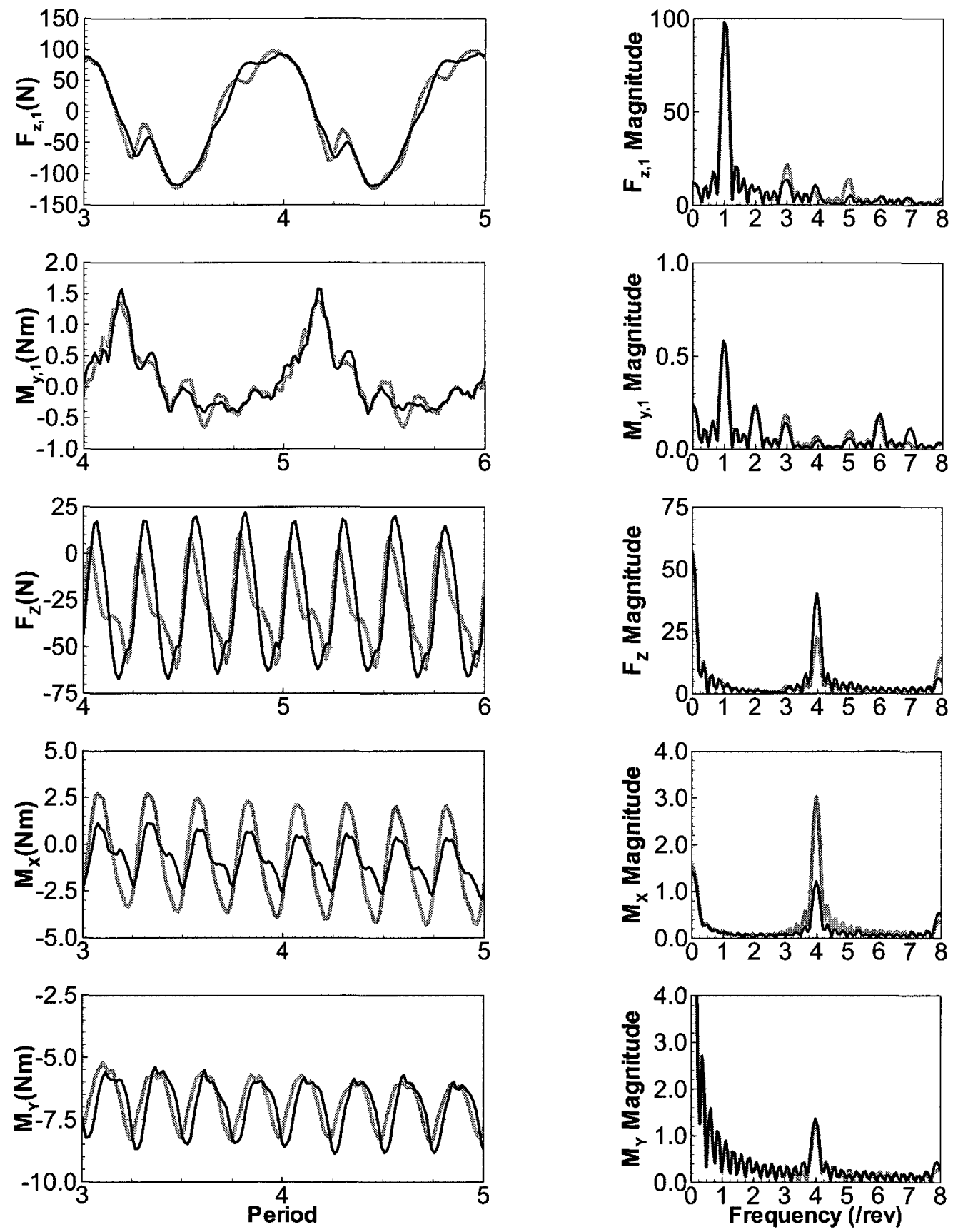

Figure 4.26: Comparison of the blade loads obtained from the GENUVP/GAST aeroelastic simulations for SHARCS blade and the softened blade when the TEF of all blades was deflected with $\phi_{\imath}=90^{\circ}$ phase shift at $1 /$ rev frequency. 

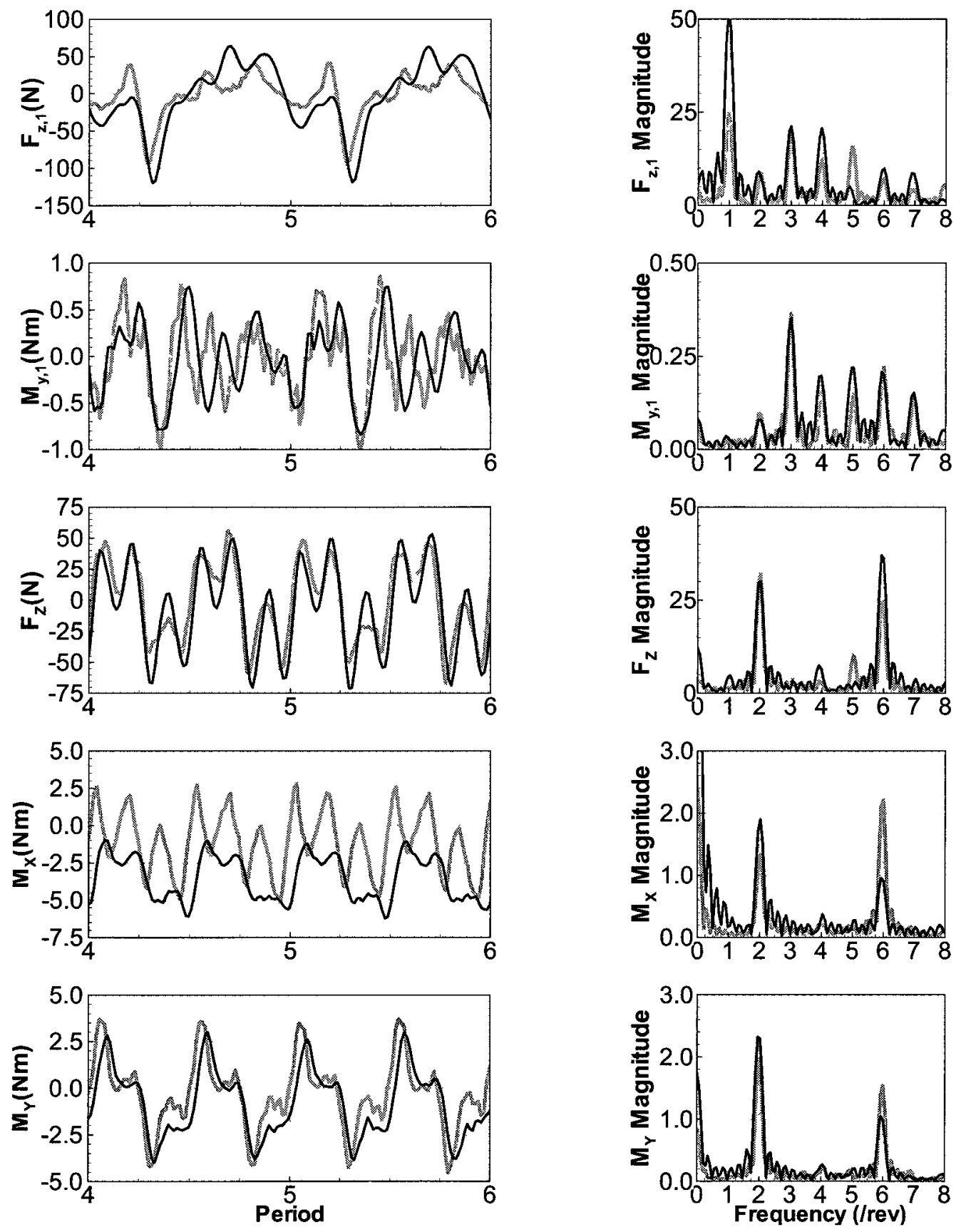

\section{SHARCS Blade} Soft Blade

Figure 4.27: Comparison of the blade loads obtained from the GENUVP/GAST aeroelastic simulations for SHARCS blade and the softened blade when the TEF of all blades was deflected with $\phi_{2}=90^{\circ}$ phase shift at $3 /$ rev frequency. 


\subsection{Conclusion}

In this chapter, the identification procedure for the discrete-time LTP state-space model of the helicopter rotor system's aeroelastic response under the TEF deflection is introduced and the validation simulations of the identified models are presented. First, the influence of the TEF deflection on the blade loads and hub loads was analyzed and presented in Section 4.2.1. It was shown that, for the baseline forward-flight regime and given helicopter rotor configuration, the suppression of vibratory hub loads, $F_{Z}, M_{X}$ and $M_{Y}$ is feasible using TEFs.

The linearity and the periodicity of the map from the TEF deflection to the rotating frame blade loads were analyzed in Section 4.3. It was shown that around the baseline forward-flight regime with an advance ratio of $\mu=0.28$, the effect of TEF deflection of one blade on the rotating frame blade loads, $F_{z}$ and $M_{y}$ of the same blade were time-periodic with periodicity of one revolution and assumed to be linear.

In Section 4.4, the simulation set up for the system identification using the subspace identification method was given. The model was considered to be a discrete-time LTP state-space model, where the zero-order-hold was assumed for the discretization. To obtain the system model at 24 azimuth locations, time-lifting was first applied to overcome the multi-sampling rate (3-to-1) of the GENUVP/GAST computer simulations and the model. Short aeroelastic runs were carried out in 26 independent simulations. A total of 430 rotor revolutions were performed in these aeroelastic runs for the identification of the strongly periodic state-space model. In fact, we only needed minimum of 192 revolutions to be completed the identification having maximum system order $n_{k}=48$ (see Eq. 3.10). Therefore, the necessary number of revolutions to identify the system model was verified to be less than those required by the parameter-based system identification method, which required from 560 to 1280 rotor revolutions with an increasing trend at higher advance ratios $[14,114]$. Therefore, the subspace identification method serves as a powerful tool to provide an initial estimate on the model set for the parameter-based system identification to decrease the time required to estimate the parameters. In $[14,114]$, the impulse responses of TEF deflections to the blade loads were taken to be over one complete period. However, according to our own analysis, the impulse responses shown to be dependent on the azimuth 
angle at which TEFs are deflected. At the advancing side of the rotor, the impulse responses die out in three revolutions for $F_{z}$ and in one revolution for $M_{y}$. At the retreading side of the rotor, the impulse responses die out in one revolution and less than a revolution for $F_{z}$ and $M_{y}$, respectively. Hence, it is strongly desirable to perform the subspace identification to obtain a reliable model set.

The discrete-time LTP state-space models were obtained in two different analyses. In the first analysis, the influence of the TEF of one blade on the other blades were ignored. Based on the model selection criteria, this analysis resulted in a model of order $n_{k}=12, \forall k=$ $1,2 \cdots 24$, with number of inputs, $m_{k}=1$ and number of outputs $p_{k}=2$. The model was validated by comparing the model output with the GENUVP/GAST simulations when the TEF of one blade was deflected with a physically realizable random input in the frequency range $0-5 / r e v$ and with single higher harmonics of rotor revolution $(1 /$ rev, $3 /$ rev, $5 /$ rev $)$.

In the second analysis, the influence of the TEF on to the aeroelastic response of the other blades was included in the system model. This resulted in a higher order system model with $n_{k}=48, \forall k=1,2 \cdots 24$, with number of inputs, $m_{k}=1$ and number of outputs, $p_{k}=8$. The aeroelastic response of the blades were compared for $\phi_{\imath}=i \frac{\pi}{2}$ phaseshifted $1 /$ rev and $3 /$ rev TEF deflections imposed to all TEFs $i=1, \cdots, 4$. It was observed that when the influence of the TEF deflection on to the other blades' loads is ignored, as performed in one blade analysis, the rotating frame blade loads cannot be predicted accurately.

More accurate predictions were obtained when the effect of TEF deflections on all blades were considered. However, this additional dynamics increased the system order. Unfortunately, the high dimensional system leads problems in the controller design process. 


\section{Chapter 5}

\section{LTP Controller Synthesis and Implementation For Helicopter Vibration Suppression}

\subsection{Introduction}

In this chapter LTP $H_{\infty}$ controller design and implementation for the helicopter vibration suppression is presented. First, the $H_{\infty}$ controller synthesis problem is formulated by selecting the design criteria. Then, the closed-loop control simulation results are given in Section 5.4. An alternative approach, $H_{2}$ controller design was carried out and the closedloop control results obtained from the implementation of $H_{2}$ controllers are discussed in Section 5.6.

\subsection{Formulation of the Controller Synthesis Problem for He- licopter Vibration Suppression}

The solution of the controller synthesis problem results in an implementable controller that makes the feedback system to satisfy the given specifications, $z_{k}$ for a given set of systems, $M_{k}$ and a set of exogenous inputs, $w_{k}$. To formulate the LTP controller synthesis problem, the inputs and outputs of the system are presented in a general block diagram representation as given in Fig. 5.1. In this figure, $P_{\text {nom }_{k}}$ corresponds to the nominal model of the helicopter aeroelastic response in terms of blade loads, $F_{z}, M_{y}$ caused by the TEF deflection, $\delta$ (see Chapter 4), and it is referred here as a plant model. Also, in this representation, the 
output disturbance models the existing baseline disturbances through the weight $W_{d \imath s t}$. The weight $W_{\text {per }}$ defines the performance objectives needed to meet for the vibration suppression. The weight $W_{a c t}$ imposes the limitation of the actuator. The noise weight, $W_{n}$ models the existing measurement noise in the system. These frequency shaped weights for the performance specifications and the existing disturbances have corresponding lifted discretetime state-space matrices, which are considered to be time-invariant.

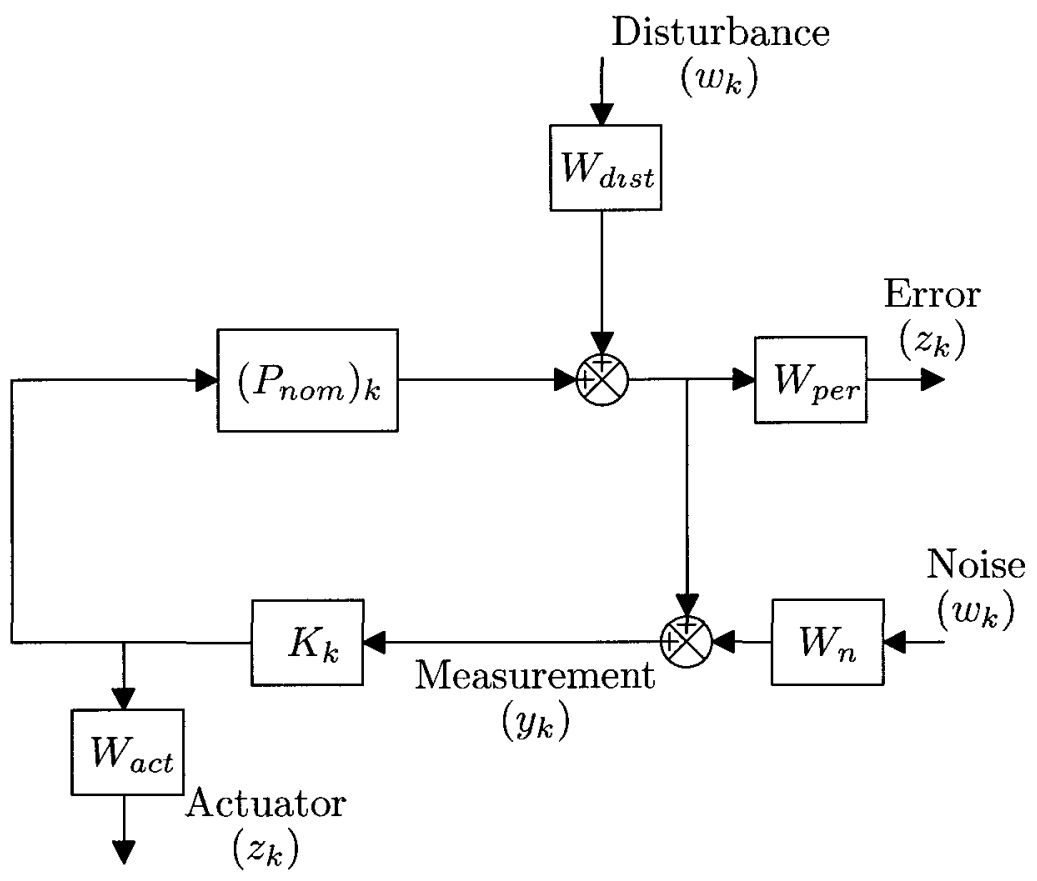

Figure 5.1: General feedback block diagram representation.

This general block diagram is then reduced to the LFT form and the system model, $M_{k}$ is obtained (see Eq. 3.24a). The system model, $M_{k}$ is common for both the $H_{\infty}$ and $\mathrm{H}_{2}$ control synthesis problems, and it is regulated to satisfy the required rank constraints (see Section 3.6.2). $M_{k}$ has $n_{M_{k}}=n_{k}+n_{p e r}+n_{d \imath s t}+n_{a c t}$ number of states, where $n_{k}, n_{\text {per }}, n_{d i s t}, n_{a c t}$ are the number of states for the nominal plant model, $P_{\text {nom }}$ and the weights, $W_{p e r}, W_{d \imath s t}, W_{a c t}$, respectively. $M_{k}$ has $m_{w_{k}}=m_{d \imath s t}+m_{\text {norse }}$ number of disturbance inputs, $n_{u_{c}}$ number of control inputs, $p_{z_{k}}=p_{p e r}+p_{a c t}$ number of performance outputs, and $p_{y_{k}}$ number of controlled outputs.

In the formulation of controller synthesis problem, two different nominal plant models 


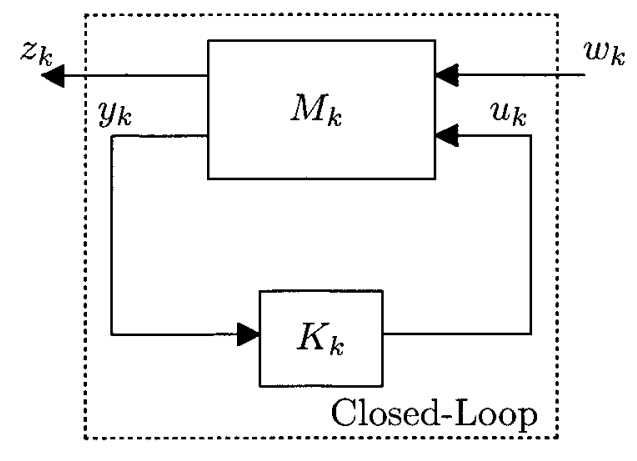

Figure 5.2: Linear fractional transformation form (repeated) [89].

are considered. The first model, obtained from the one blade analysis, ignores the interactions of the blades (Section 4.5). The second model, obtained from the all blade analysis, takes into account the interactions of the blades through the disturbed wake (Section 4.6). There are certain limitations of using these models in controller synthesis. It was shown in Section 4.5 that the one blade analysis induces erroneous results when all the TEFs are deflected. Specifically, the helicopter rotor system response at the $3 /$ rev and $5 /$ rev frequencies cannot be predicted accurately when the blade interactions are ignored. This means that the controller is prone to generate unexpected results and even cause instability of the closed-loop system due to the unmodelled system dynamics. One way of preventing instability in the closed-loop system is to introduce an uncertainty in the nominal plant model around these frequencies, which unfortunately increases the order of the system model, $M_{k}$. The second way is to use the nominal plant model obtained from the all blade analysis, which also increases the order of the system model, $M_{k}$. High dimensional system models increase the number of decision parameters in a control synthesis problem (see Eq. 3.31). In the optimization computation, performed using Matlab v.7.11.0, memory problems were then encountered.

\subsubsection{Controller Synthesis Problem Formulation: One Blade Analysis}

Recall that the one blade analysis resulted in a discrete-time LTP state-space model from input $u_{k}=\delta_{1}$, TEF deflection to outputs $y_{k}=\underline{F}_{z, 1}, \underline{M}_{y, 1} \forall k=1, \cdots, N$, the rotating frame blade loads of one blade for one full revolution. These outputs were time-lifted to overcome 
the sampling rate differences between the TEF input and the helicopter aeroelastic response (3-to-1). This results in lifted outputs, $\underline{F}_{z}, \underline{M}_{y}$ with $p_{k}=2(\times 3)$ number of outputs and $m_{k}=1$ number of inputs $\forall k=1, \cdots, N$.

$$
\left[\begin{array}{c}
x_{k+1} \\
y_{k}
\end{array}\right]=\left[\begin{array}{cc}
A_{k} & B_{k} \\
C_{k} & D_{k}
\end{array}\right]\left[\begin{array}{l}
x_{k} \\
u_{k}
\end{array}\right]
$$

where

$$
\begin{array}{ll}
A_{k+N}=A_{k} \in \mathbb{R}^{n_{k} \times n_{k}}, & B_{k+N}=B_{k} \in \mathbb{R}^{n_{k} \times m_{k}} \\
C_{k+N}=C_{k} \in \mathbb{R}^{p_{k} \times n_{k}}, & D_{k+N}=D_{k} \in \mathbb{R}^{p_{k} \times m_{k}}
\end{array}
$$

For the suppression of vibratory rotating frame blade loads, the nominal system was chosen to have one control input, $\delta_{1}$ and two controlled outputs, $\underline{F}_{z, 1_{T}}, \underline{M}_{y, 1_{T}}$, which are the total rotating frame blade loads. In this formulation, the interaction of the blades were ignored, and the designed controller, $K_{k}$ would have the same periodicity of the system $\left(N_{K}=N\right)$. The order of the controller would be equal to the order of the system model in LFT form $\left(n_{K_{k}}=n_{M_{k}}\right)$.

For the suppression of vibratory hub loads, the system model was augmented to form a four-blade analysis. The output of all the blades were first transferred from the rotating frame to the non-rotating frame according to their azimuth location, $\Psi_{k}$ and then summed to obtain the hub loads. The vibratory baseline disturbances were introduced either in the rotating frame and/or in the non-rotating frame to obtain the total hub loads, $\underline{F}_{Z_{T}}, \underline{M}_{X_{T}}, \underline{M}_{Y_{T}}$ to be controlled. The resultant nominal plant model, $P_{n o m_{k}}$ is periodic with a period $N$ and it has control inputs from the TEF of all blades, $\delta_{1,2,3,4}$ and controlled outputs $\underline{F}_{Z_{T}}, \underline{M}_{X_{T}}, \underline{M}_{Y_{T}}, p_{k}=3(\times 3) \forall k=1, \cdots, N$. The, resultant controller, $K_{k}$ is also $N$-periodic.

In this formulation, the blades are not interacting, however, since the controller's objective is to suppress the total vibratory hub loads, caused by all blades, the control command to deflect the TEF of each blade is not independent from each other. In addition, the required TEF deflections are obtained based on the hub loads, rather than rotating frame blade loads. 


\subsubsection{Controller Synthesis Problem Formulation: All Blades Analysis}

In the all blade analysis, the effect of the TEF deflection of one blade on to other blades caused by the disturbance in the wake was included in the system model. The resultant discrete-time LTP state space model has one input, $u_{k}=\delta_{1}$ and time-lifted outputs $y_{k}=\underline{F}_{z, 1,2,3,4}, \underline{M}_{y, 1,2,3,4}, p_{k}=8(\times 3)$ number of outputs $\forall k=1, \cdots, N$.

For the suppression of both vibratory hub loads, and rotating frame blade loads the system model was augmented to form a four-blade analysis as in Eq. 4.6. For the rotating frame blade load suppression, the direct output of the system was used. For the suppression of hub loads, the total rotating frame blade loads were calculated then were transferred from the rotating frame to the non-rotating frame according to their azimuth location, $\Psi_{k}$. The transferred loads were then summed to obtain the hub loads. The vibratory baseline disturbances were introduced either in the rotating frame and/or in the non-rotating frame. The resultant nominal plant model, $P_{n o m}$ is periodic with a period $N$ and it has control inputs $m_{k}=4, \forall k=1, \cdots, N$ from the TEF of all blades and controlled outputs $\underline{F}_{Z_{T}}, \underline{M}_{X_{T}}, \underline{M}_{Y_{T}}, p_{k}=3(\times 3) \forall k=1, \cdots, N$. The controller $K_{k}$ has the same periodicity of the plant model $\left(N_{K}=N\right)$.

Interactions of the blades are included in the formulation and, since the controller's objective is to suppress the total vibratory hub loads caused by all blades, the control command to deflect the TEF of each blade is dependent on every other.

\subsection{Disturbance Modeling and Performance Selection}

We aimed to suppress the helicopter's vibratory hub loads, $F_{Z}, M_{X}, M_{Y}$ by controlling the rotating frame blade loads. For identical blades having the same structural properties and experiencing the same aerodynamic characteristics, the integer multiples of $N_{b} / r e v$ hub loads at the hub vertical shear is caused by the integer multiples of $N_{b} /$ rev rotating frame vertical blade loads. For a four-bladed rotor system, only the harmonics of $N_{b} / r e v$ vibratory vertical loads at the rotating frame of each blade $\left(F_{z, 2}\right)$ are in phase; the other harmonics cancel out due to the phase differences between them. Therefore, to suppress, for example, the $4 /$ rev and $8 /$ rev vertical hub load, $F_{Z}$, it is necessary to suppress the $4 / r e v, 8 / r e v$ 
components of the vibratory vertical blade loads, $F_{z, \imath}$ in the rotating frame. Alternatively, it is necessary to create a phase difference at $N_{b} / r e v$ frequency between the blades to cancel each other out in the non-rotating frame.

To suppress the $4 / r e v, 8 / r e v$ vibratory hub roll and pitch moments, $M_{X}, M_{Y}$, it is necessary to suppress the $3 / r e v, 5 / r e v$ and $7 / r e v, 9 /$ rev vibratory rotating frame flap bending moment, $M_{x, \imath}$ and pitching moment, $M_{y, \imath}$. Because the transformation of the rotating frame blade loads with a frequency content of $3 / r e v, 5 /$ rev and $7 /$ rev, $9 /$ rev induces $4 /$ rev, $8 /$ rev non-rotating frame hub roll and pitch moments for an identical four-bladed rotor system. For this specific application (i.e. structural design and the selected flight condition), both the $3 / r e v$ and $5 / r e v$ frequency component of the rotating frame flap bending moment had higher contribution to the hub roll and pitch moments, $M_{X}, M_{Y}$. In addition, to maintain the same trim state, the $1 /$ rev component of rotating frame flap bending and pitching moments had to be balanced.

Two approaches were considered in the controller design. The first approach aimed to suppress the blade loads in the rotating frame. In this design, all the weights described in Section 5.2 were the same for all blades, and the performance weight was selected to decrease vibrations at some specific frequency. This type of approach guarantees that the resultant controller is phase shifted, however, it does not guarantee that the hub loads would be suppressed.

In the second approach, suppression of blade loads and/or hub loads was considered by performing a four-blade analysis. In the controller synthesis problem formulation, either the baseline disturbances can be given to each blade in the rotating frame or $N_{b} / r e v$ disturbances can be introduced in the non-rotating frame. The former necessitates the measurement of rotating frame blade loads or hub loads, while the latter requires the measurement of the hub loads. To satisfy the rank constraints of the $H_{2}$ controller design method (See Section 3.6.2), regularization is needed [50]. If only the hub load disturbances are introduced in the non-rotating frame, the controller is prone to fail since it cannot detect the source of the vibration. If the baseline disturbances are given to each blade, then the phase shift between the disturbances should be included in the disturbance weight. 


\subsection{LTP $H_{\infty}$ Controller Synthesis and Closed-Loop Control Simulations}

\subsubsection{One Blade Analysis: Suppression of the Rotating Frame Blade Load, $F_{z}$ at $1 /$ rev}

In the controller synthesis, the state-space model obtained from the one blade analysis was used as a nominal plant model, $P_{n o m}$. The performance weight, $W_{p e r}$ was chosen to minimize the rotating frame vibratory load, $F_{z, 1}$ at $1 /$ rev frequency. A high pass secondorder LTI filter was used to model $W_{\text {act }}$ to increase the weight of the TEF excitations over a specific frequency in the optimization. The weight $W_{n}$ was taken to be constant. It models the existing noise in measurement with signal-to-noise ratio of 1\%. Figure 5.3 gives the selected performance criteria, $W_{p e r}$ and $W_{a c t}$, and the disturbance weight, $W_{d s s t}$ for the suppression of the $1 / r e v$ vibratory rotating frame load, $F_{z}$.
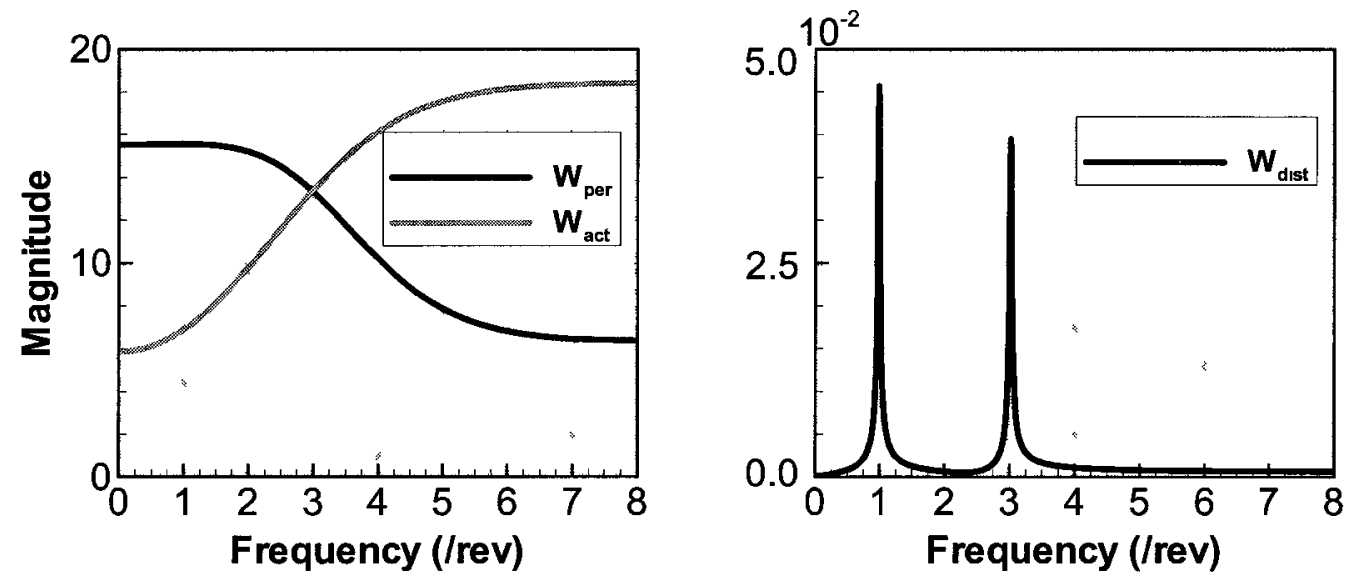

Figure 5.3: Control of $1 /$ rev rotating frame blade load, $F_{z} \cdot H_{\infty}$ controller design performance and disturbance weights.

These weights were substituted in the general feedback diagram then reduced in to LFT form to obtain the system matrix, $M_{k}$. For this system matrix, the theory given in Section 3.6.1 was first applied to design a discrete-time LTI $H_{\infty}$ controller. However, the optimization problem did not provide any feasible solution; i.e there is no LTI controller 
that satisfy the stability and performance criteria of the closed-loop system.

For the same system matrix, $M_{k}$ the optimization problem resulted in a LTP controller of order $n_{K_{k}}=18, \forall k=1, \cdots, N$. The closed-loop simulations were performed for the LTP controller both using the plant model of higher order $\left(n_{k}=24\right)$, and the GENUVP/GAST aeroelastic computer code. The controlled and uncontrolled rotating frame blade loads, $F_{z}$ and $M_{y}$ were compared for the blade number one and blade number three. The control commands given to the TEF's, $\delta_{1}, \delta_{3}$ are presented in Fig. 5.4. The GENUVP/GAST aeroelastic simulations resulted in higher flap deflections to control the $F_{z}$ as compared to the reduced-order model simulations. Less than 2-degrees peak-to-peak TEF deflections were required to reduce the $1 /$ rev component of the rotating frame vertical load, $F_{z}$ by $65 \%$.
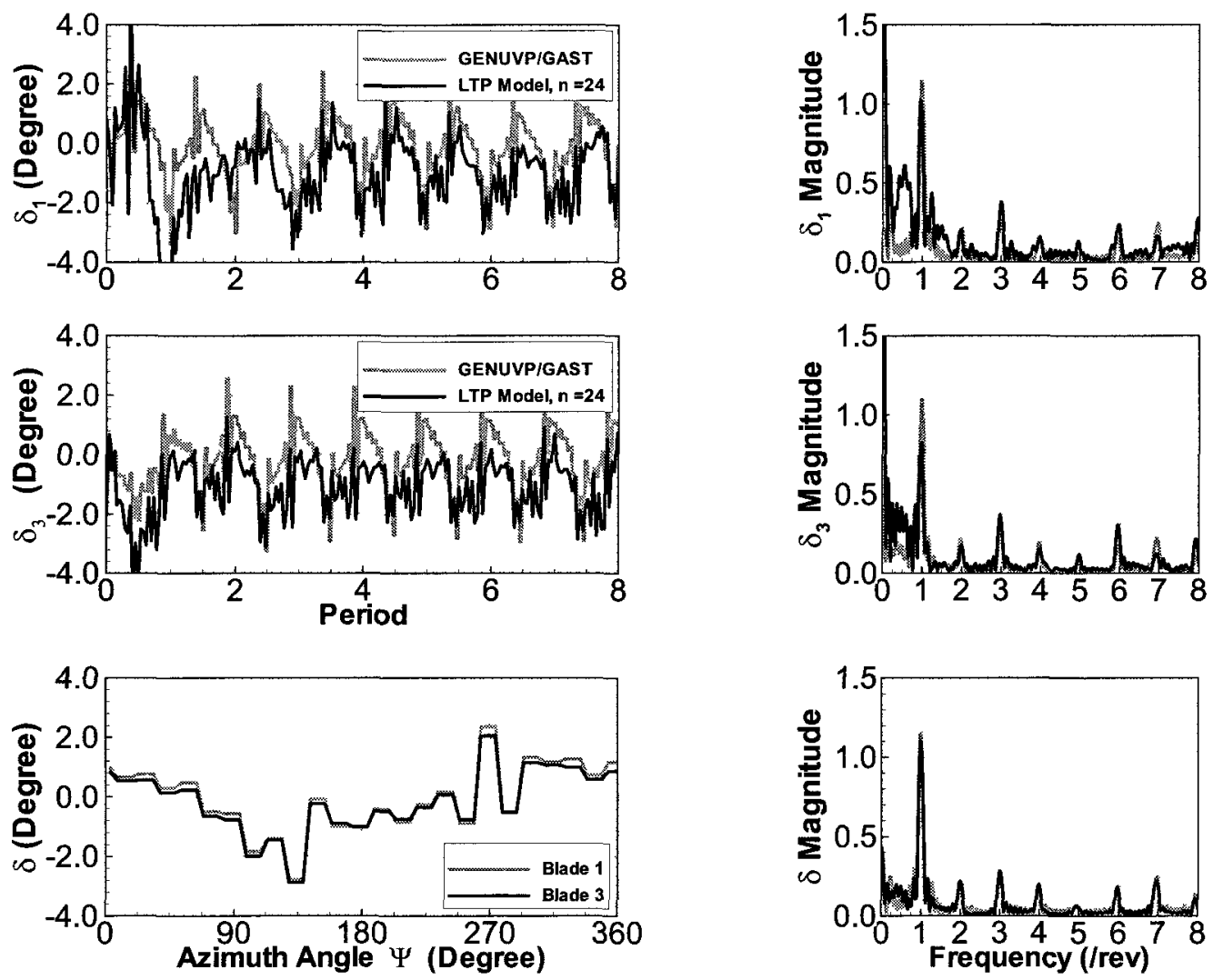

Figure 5.4: Control of 1/rev rotating frame blade load, $F_{z}$. Control command given to the TEFs $\delta_{1}$ and $\delta_{3}$ by the $H_{\infty}$ controller. 
The comparison between the uncontrolled and controlled baseline rotating frame blade loads and hub loads is presented in Fig. 5.5 and Fig. 5.6, respectively. Figure 5.5 shows that $65 \%$ reduction at the $1 /$ rev frequency content of $F_{z}$ in all blades did not cause any detrimental excitations of higher harmonics. Less than $20 \%$ increase in $4 / r e v$ and $5 / r e v$ frequency content of $F_{z}$ was observed. 

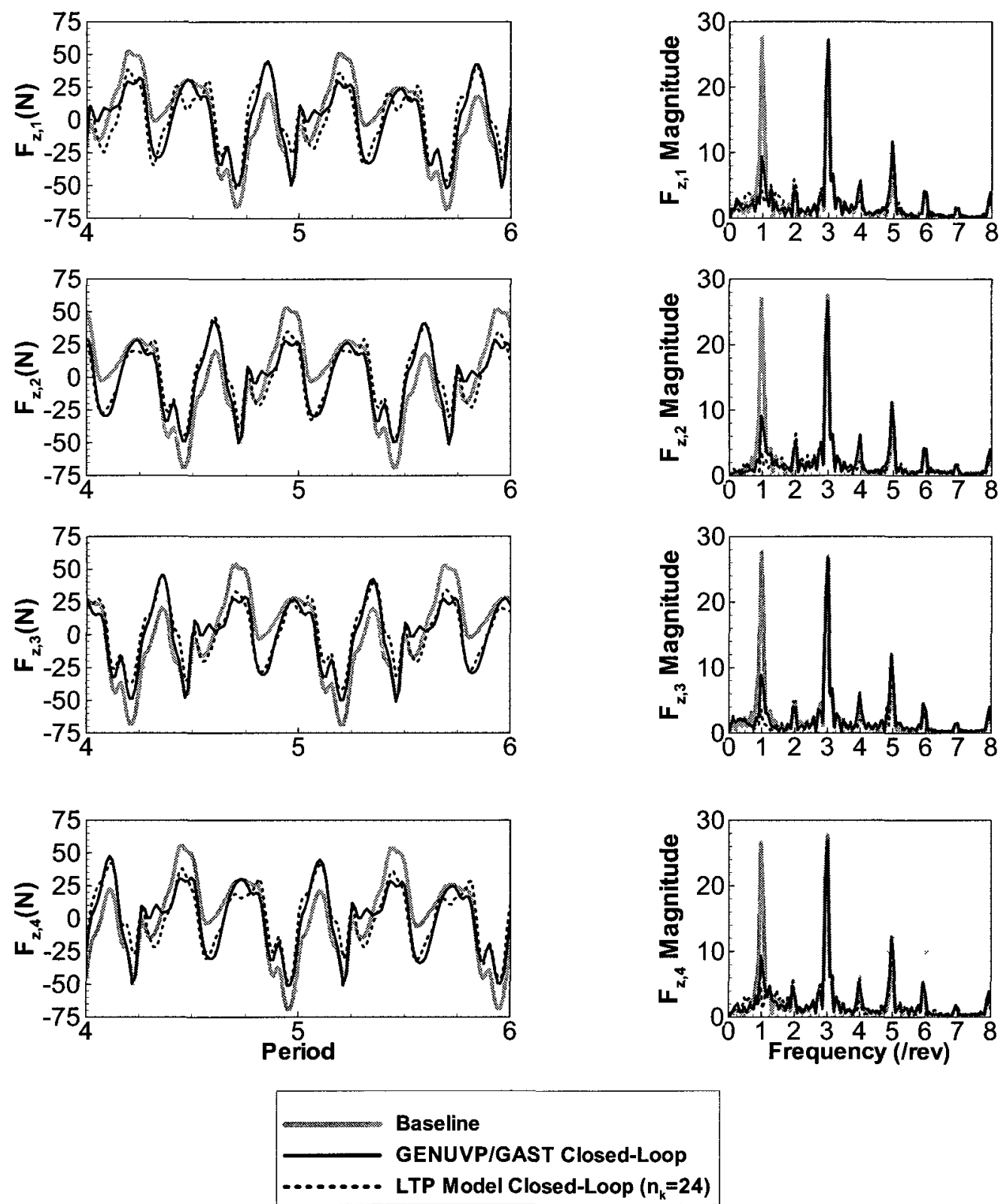

Figure 5.5: Control of $1 /$ rev rotating frame blade load, $F_{z}$. Closed-loop control simulations: comparison between the baseline and controlled blade loads, $F_{z}$ obtained from the reducedorder model and the GENUVP/GAST simulations. 

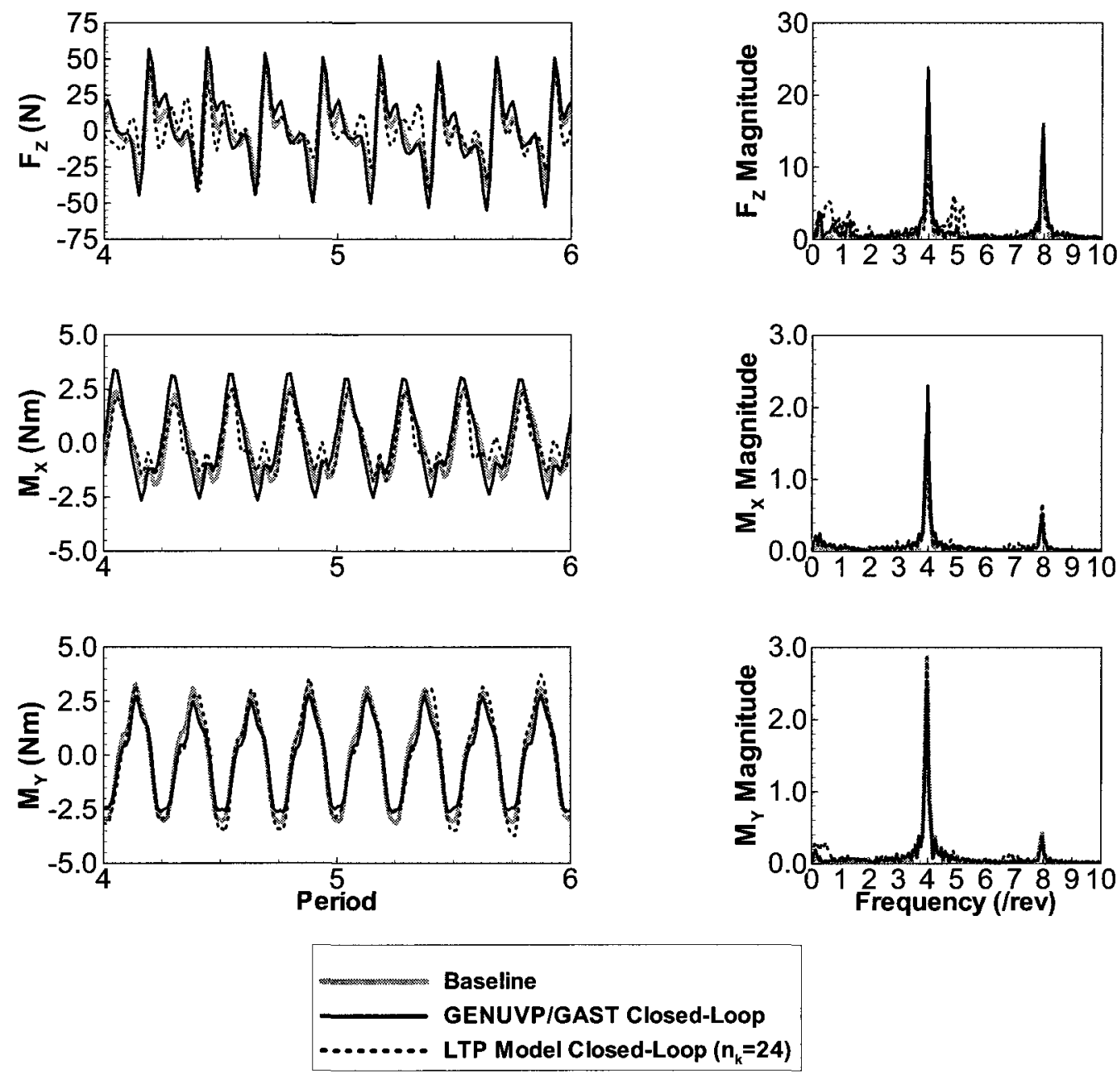

Figure 5.6: Control of 1/rev rotating frame blade load, $F_{z}$. Closed-loop control simulations: comparison between the baseline and controlled hub loads, $F_{Z}, M_{X}$, and $M_{Y}$ obtained from the reduced-order model and the GENUVP/GAST simulations.

Since, $1 /$ rev frequency content of $F_{z}$ was reduced for all blades, the effect of this reduction was not observed at the non-rotating frame due to the phase difference at $1 /$ rev frequency of each blade. However, the excitation of $5 / r e v$ frequency content of the vertical force decreased the hub pitch moment, and increased the hub roll moment at $4 / r e v$ frequency. This effect was previously mentioned in [14].

To improve the controller's performance, more accurate system representation obtained 
from the all blade analysis should be used. In addition, the minimization of hub loads, $F_{Z}, M_{X}$ and $M_{Y}$ should be included in performance objectives. However, each of these improvements caused implementation problems, which are listed as follows.

\subsection{Implementation Problems and Propositions}

The controller design problems for this specific application are listed as follows.

1. Computational Problems: Discrete-time LTP $H_{\infty}$ controller synthesis using LMIs for LTP systems causes implementation problems for complex systems having high dimension and long period. Since the current LMI solvers are roughly cubic in the decision variables, the controller design is computationally intensive [104].

2. System Model Problems: There were two system models obtained in Chapter 4 . While the one blade analysis ignores the interaction of the blades, the all blade analysis takes into account the interaction of the blades through the disturbed wake. The unmodelled dynamics caused by the disturbance of the wake if not introduced in the controller synthesis as model uncertainty can cause performance degradation and even instabilities in the closed-loop system. Even including only the effect of the TEF deflection on the blade directly under the influence of the disturbed wake, the computational problems cannot be avoided.

Possible solution methods related to implementation of the discrete-time LTP $H_{\infty}$ controller design are listed as follows.

1. Riccati Based Solution to $H_{\infty}$ Control Problem: Discrete-time periodic $H_{2}$ and $H_{\infty}$ controller synthesis problems can be formulated using two discrete-time periodic Riccati Equations. However, the solution of the discrete-time periodic Riccati Equation requires computational tools, which are not accessible at the moment [109]. 


\section{System Model Conversion:}

- Time-lifting can be applied to the discrete-time LTP state-space model to obtain the discrete-time LTI representation. In this case, the controlled variables are measured over a full revolution and the controller outputs are the TEF deflections that are applied at each azimuth angle in the next full revolution. The time-lifted formulation of a time-periodic system and design of a LTI controller for a full revolution was previously implemented for helicopter control applications $[9,56]$. In addition, the numerical instabilities [76] and control implementation problems [75] associated with the lifted system were already mentioned. Therefore, in this thesis, this approach will not be revisited.

- The system model can be transferred to continuous-time, and expressed as a summation of harmonics of $n / r e v, n=0,1, \cdots$. The controller structure can be written as in Eq. 5.3 and the controller matrices $K_{ \pm n} \forall n=0,1, \cdots$ can be solved using optimization.

$$
K=K_{0}+K_{ \pm 1} e^{ \pm j \Omega t}+\cdots+K_{ \pm n} e^{ \pm j r w t}
$$

\section{Structure of Controller:}

- This is specifically applicable when all blades are augmented as in Eq. 4.6. It is necessary to explore the possibility of defining a structure to the decision variable matrices, $X_{k}, Y_{k}$ in order to decrease the number of decision variables. In the nominal plant model representation the blades were represented in a block diagonal form, where the blades are uncoupled. However, the controlled output (hub loads) are obtained from the response of all blades. Hence the system model $\left(M_{k}\right)$ is coupled. The question arises: is it possible to uncouple the controllers, or would it be meaningful to uncouple them by defining a structure to the $X_{k}, Y_{k}$ matrices?.

- The controller periodicity can be taken different than the system's periodicity in order to decrease the number of decision variable matrices, $X_{k}, Y_{k}$. For a $N$ periodic system, an $N_{K}$-periodic controller, where $N_{K}<N$, can be designed [52]. 
For $N_{K}=1$, the controller is reduced to a LTI controller. This method, of course, causes performance degradation since the system response is different at each azimuth angle. Gain scheduling can also be applied if multiple LTI controllers are designed to accommodate the change in the system dynamics.

4. Different Controller Design Approach: By using the high dimensional system model, surrogate-based optimization can be carried out to minimize the hub loads. This method was indeed applied to obtain the swash plate settings to satisfy the wind tunnel trim state in the GENUVP/GAST aeroelastic code at different advance ratios.

In light of the proposed solution methods, we proceed with the development of an inhouse LTP Riccati solver to design $H_{\infty}$ controllers. However, since the solution of $H_{\infty}$ controller requires the coupled solution of two Riccati Equations with a search on optimization parameter, $\gamma$, the solver was first tested to design $\mathrm{H}_{2}$ controllers. Further modifications in the in-house TP Riccati solver to design $H_{\infty}$ controllers should be considered as future work.

\subsection{LTP $\mathrm{H}_{2}$ Controller Synthesis and Closed-Loop Control Simulations}

The computational problems limited the design of time-periodic $H_{\infty}$ controllers using LMIs. Therefore, the aim was at designing a time-periodic $H_{2}$ controller from the solution of two discrete-time periodic Riccati Equations [48]. The algorithm given in Section 3.7 was applied first to design the estimator and then the controller. In the controller design, the system models obtained from one blade analysis and all blade analysis were used (see Fig. 5.1). Different performance specifications were imposed to address the specific frequency content of the rotating frame and hub loads. In addition, the disturbance weight was considered time-invariant and the same for all blades, but time-invariant and phase shifted in respect to the blade azimuth angle. The results presented in the following sections are organized based on plant models, performance specifications, and disturbance weight selection.

The closed-loop system characteristics were analyzed by comparing the time and frequency responses of uncontrolled and controlled rotating frame blade and hub loads obtained 
from both GENUVP/GAST simulations, and reduced-order models.

\subsubsection{One Blade Analysis: Suppression of the Rotating Frame Blade Load, $F_{z}$ at $1 /$ rev}

The system model obtained from the one blade analysis was used as a plant model in the feedback configuration given in Fig. 5.1. The performance weight for the measured output was selected to minimize the vibratory rotating frame blade load, $F_{z}$ in the frequency range $0-5 / r e v$ and the actuator weight was selected to prevent high frequency excitation of TEFs. In addition, a low-pass filter was introduced in the general feedback diagram to prevent the excitation of higher harmonics caused by the discretization of the system. Since, the $\mathrm{H}_{2}$ controller design is sensitive to plant and environment modeling, the disturbance weight was selected to include the baseline disturbances at the $1 /$ rev, $\cdots, 5 /$ rev frequencies (see Fig. 5.7). The selected weights were substituted in the general feedback diagram, and then the LFT form (see Fig. 5.2) was obtained. Two discrete-time periodic Riccati Equations corresponding to the estimator and controller design were solved using an in-house solver. The order of the resultant controller is $n_{K_{k}}=25$. A closed-loop system matrix was formed and the $\mathrm{H}_{2}$-norm of the open-loop and closed-loop system matrices were calculated by using Eq. 3.37b. The controller reduces the $H_{2}$-norm of the closed-loop system by $52 \%$ weighted at the $1 /$ rev frequency. 

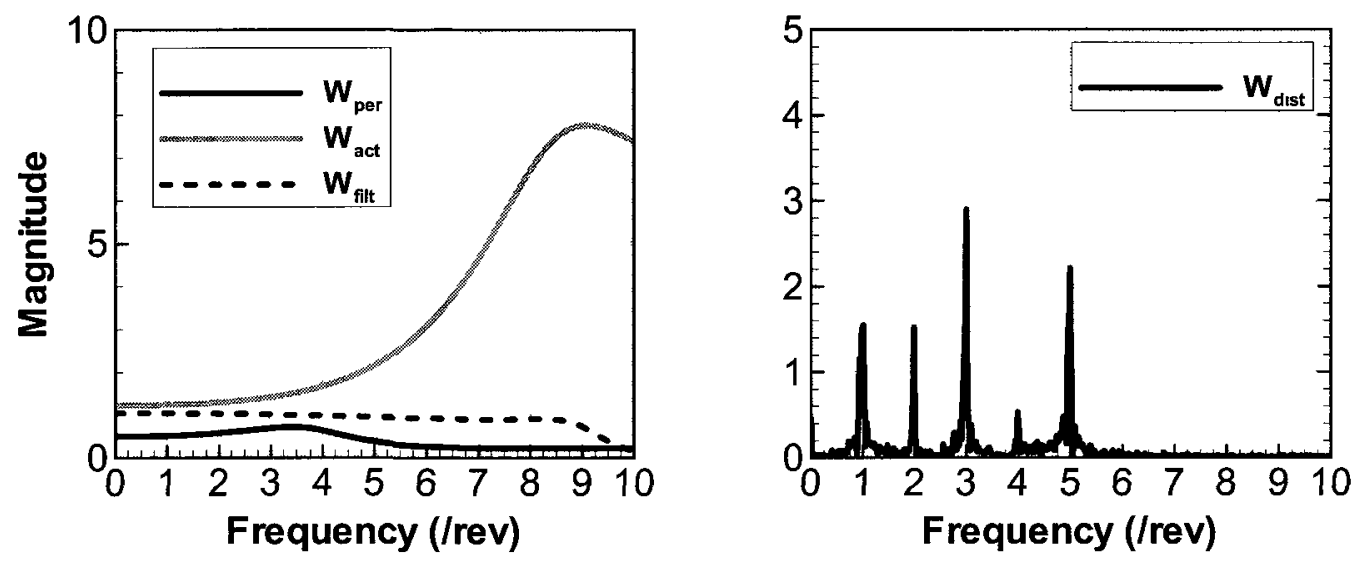

Figure 5.7: Control of 1/rev rotating frame blade load, $F_{z} . H_{2}$ controller design performance and disturbance weights.

The $H_{2}$ controller was applied in the closed-loop configuration and simulations were performed both using the GENUVP/GAST and the reduced-order model $\left(n_{k}=24\right)$. The TEF deflections reach a steady-state value in one revolution. Figure 5.8 shows the control TEF deflections for blades one and three obtained from the closed-loop GENUVP/GAST computer simulations and the simulations carried out with the reduced order model $\left(n_{k}=24\right)$. The reduced-order model simulations predicts smaller 3/rev TEF deflections as compared to GENUVP/GAST simulations. Since the 1/rev TEF deflection applied by the controller excites the 3/rev $F_{z}$ in GENUVP/GAST simulations, which is fed back into the system, this effect is not captured by the one blade analysis. In Fig. 5.8, the TEF deflections obtained from the GENUVP/GAST simulations for blades one and three are presented with respect to their azimuth location when the blades were in their fourth revolution. As the phase shifted controller matrices $\left(K_{\imath_{k}}=K_{k+(\imath-1) \frac{N}{N_{b}}}, \imath=1, \cdots, N_{b}\right)$ were used for all other blades, the steady-state TEF control deflections were almost equivalent. 

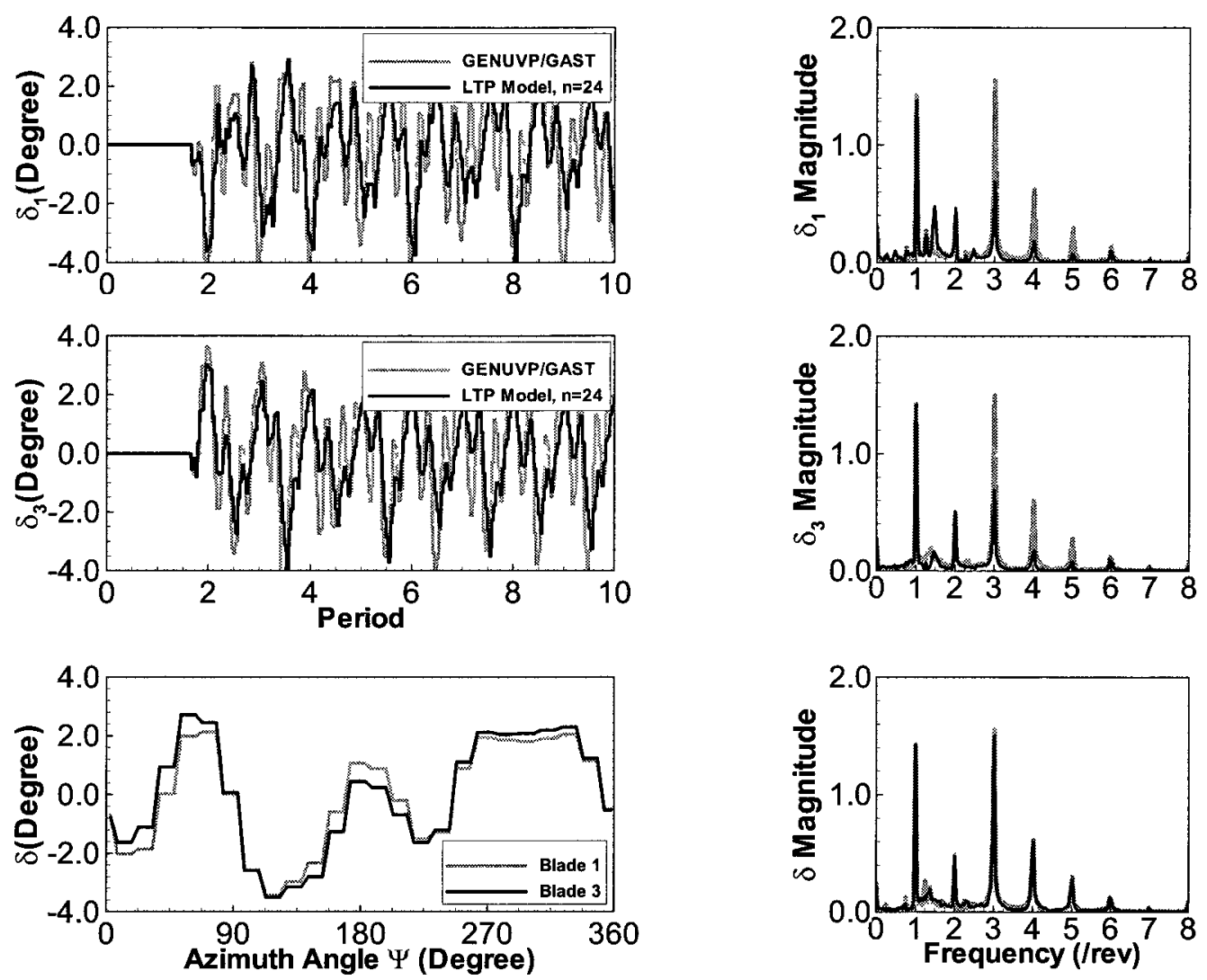

Figure 5.8: Control of $1 /$ rev rotating frame blade load, $F_{z}$. Control commands given to TEF $\delta_{1}$ and $\delta_{3}$ by the $H_{2}$ controller.

The results of closed-loop simulations are summarized in Fig. 5.9 and 5.10. In Fig. 5.9 the frequency responses obtained from open-loop and closed-loop control simulations for the rotating frame vertical blade loads, $F_{z, 1,2,3,4}$ for all blades are presented. 

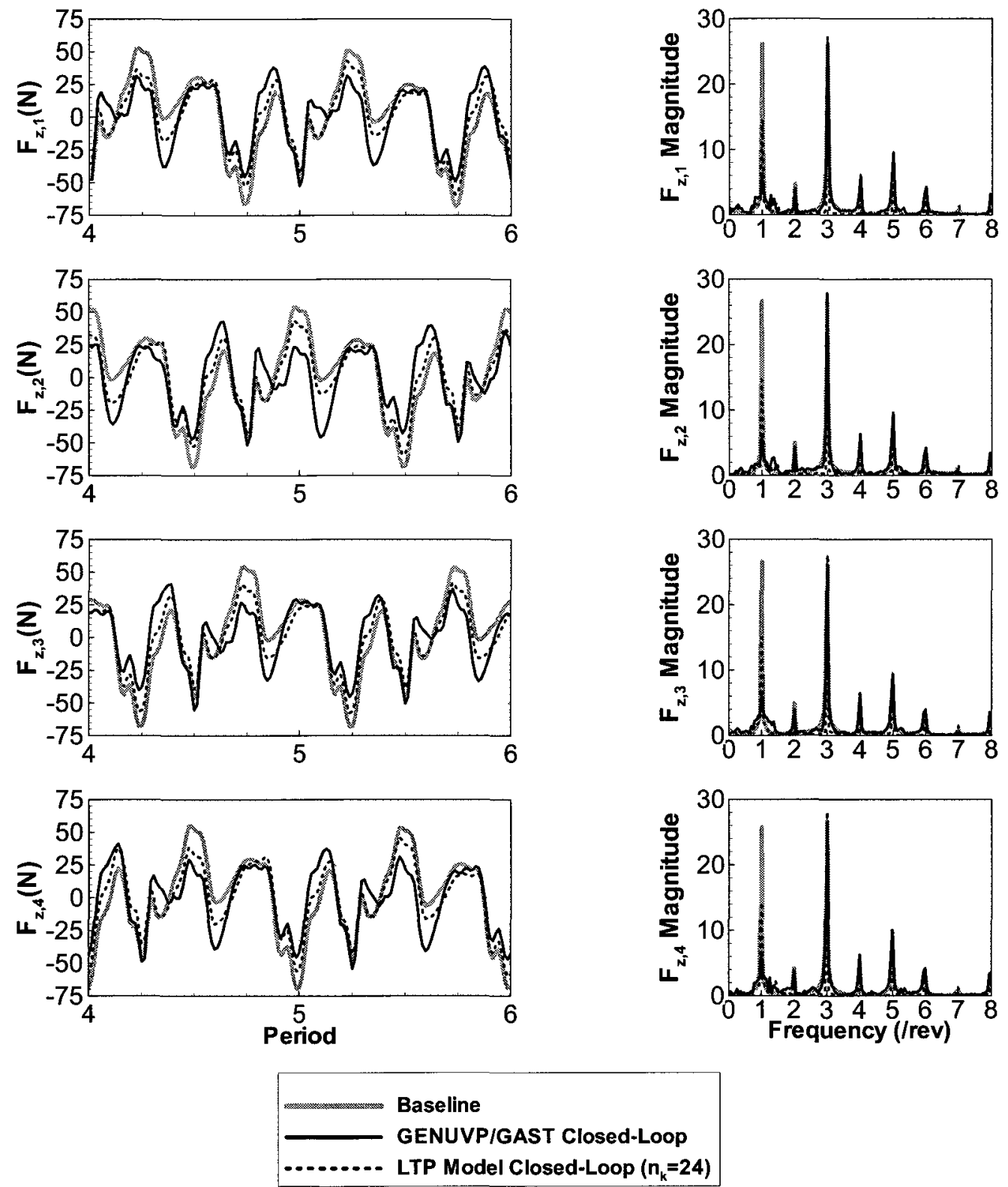

Figure 5.9: Control of 1/rev rotating frame blade load, $F_{z}$. Closed-loop control simulations: comparison between the baseline and controlled blade loads, $F_{z}$ obtained from the reducedorder model and the GENUVP/GAST simulations. 
The amplitude of the vertical rotating frame blade load, $F_{z}$ was reduced $89 \%$ at the 1/rev frequency at each blade with TEF deflections less than 4 degrees peak-to-peak value, as defined by the performance criteria. The controller also affected the dynamics at higher harmonics. At the $2 / r e v$ and $3 /$ rev frequencies $22 \%$ and $7 \%$ reductions were recorded, respectively. The $F_{z}$ load was excited at the $4 /$ rev, $5 /$ rev and $6 / r e v$ frequencies by $63 \%, 42 \%$ and $21 \%$, respectively. The amplitude of $F_{z}$ was excited at the $8 / r e v$ frequency by $39 \%$, and reduced at the $7 /$ rev and $9 /$ rev frequencies by $55 \%$ and $22 \%$, respectively. The $L_{2}$ norm of the measured rotating frame vertical blade load $F_{z}$ was reduced by $22.8 \%$ as compared to the uncontrolled baseline flight condition. The rotating frame blade pitch moment, $M_{y}$ was suppressed at the $1 /$ rev and $2 /$ rev frequencies by $20 \%$ and $17 \%$, respectively. At the $3 / r e v$ frequency, the pitch moment was excited by $37 \%$ but it was suppressed at the $5 / r e v$ and $6 /$ rev frequencies by $42 \%$ and $56 \%$, respectively. 

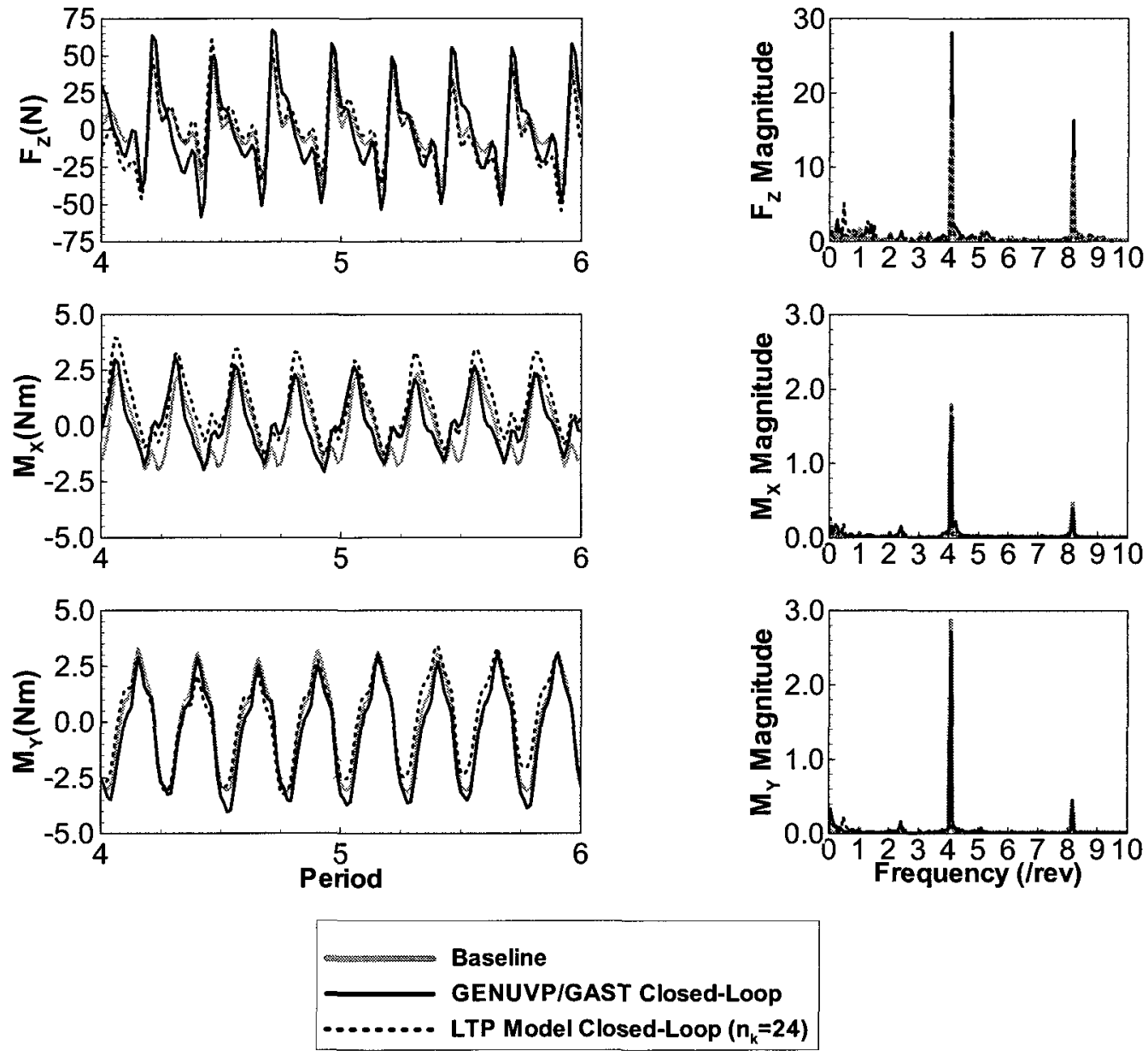

Figure 5.10: Control of $1 /$ rev rotating frame blade load. $F_{z}$. Closed-loop control simulations: comparison between the baseline and controlled hub loads, $F_{Z}, M_{X}$ and $M_{Y}$ obtained from the reduced-order model and the GENUVP/GAST simulations.

Since phase shifted controller $\left(K_{\imath_{k}}=K_{k+(\imath-1) \frac{N}{N_{b}}}, i=1, \cdots, N_{b}\right)$ was used for all other blades, the suppression levels at the frequencies $1 /$ rev, $2 /$ rev $, \cdots, 12 /$ rev resulted to be the same for all blades. Therefore, the vibratory hub loads have only the $N_{b} / r e v$ frequency content. The excitation of the $4 / \mathrm{rev}, 8 / \mathrm{rev}$ frequencies in the rotating frame resulted in an excitation of the vertical force in the hub frame by $69 \%$ and $46 \%$, respectively. The hub roll moment, $M_{X}$ was reduced at the $4 /$ rev, $8 /$ rev frequencies by $12 \%$ and $31 \%$, respectively and the hub pitch moment, $M_{Y}$ was reduced by $5 \%$ and $18 \%$, respectively. There was no 
significant change in the hub loads, $F_{X}, F_{Y}$ and $M_{Z}$.

For the $H_{\infty}$ controller (see Fig. 5.5), similar vibration reduction levels were obtained. However, the $\mathrm{H}_{2}$ controller synthesis necessitates detailed disturbance modeling, which increases the order of the controller. The time required to solve for the optimal controller was decreased from $3-4$ days to less than ten minutes for augmented model size of similar order of magnitude in LFT form when the $H_{\infty}$ design was taken. However, it should be noted that, the solution of the $H_{\infty}$ controller synthesis problem requires search of the optimal $\gamma$ value, which is not necessary for the $H_{2}$ controller synthesis.

\subsubsection{All Blade Analysis: Suppression of the Rotating Frame Blade Load, $F_{z}$ at $4 /$ rev}

The system model obtained from the all blade analysis $\left(n_{k}=48\right)$ in the four-blade configuration was used as the plant in the feedback diagram given in Fig. 5.1. The performance weight for the measured output was selected to minimize the vibratory rotating frame blade load, $F_{z}$ specifically at the $4 /$ rev frequency. The disturbance weight included the baseline disturbances in the frequency range $0-5 / r e v$, and it was taken to be the same for all blades, i.e. with no phase difference. In Fig. 5.11 the selected weights are presented. The resultant controller's order is $n_{K_{k}}=262$. 

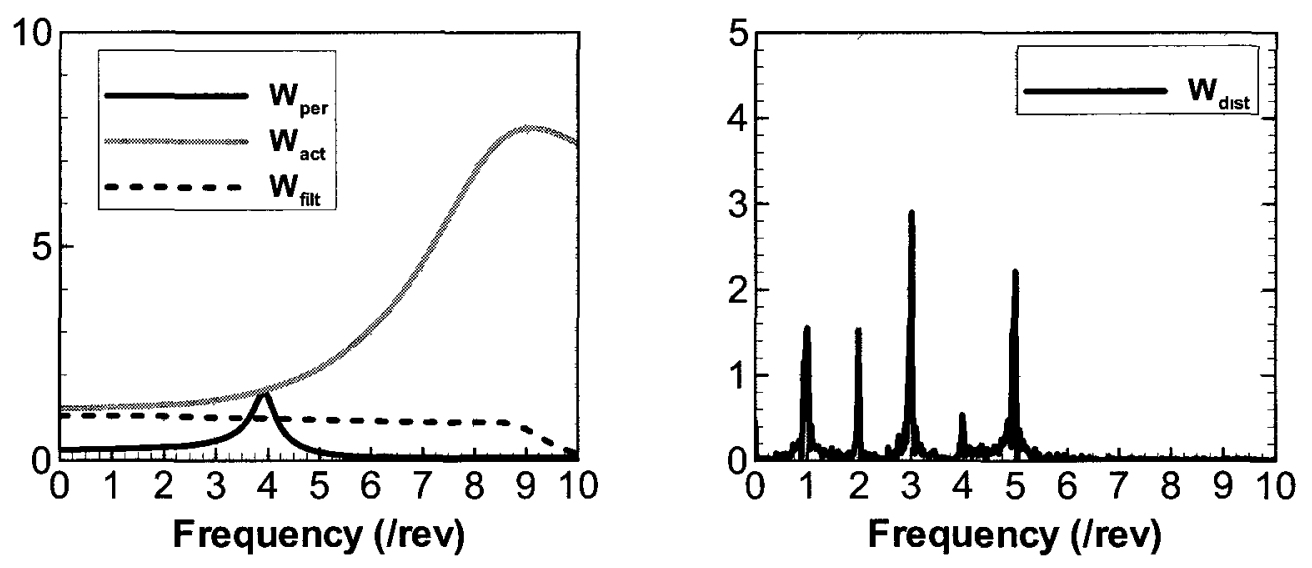

Figure 5.11: Control of 4/rev rotating frame blade load, $F_{z} \cdot H_{2}$ controller design performance and disturbance weights.

The $H_{2}$ controller was applied in the closed-loop configuration and simulations were performed both the GENUVP/GAST and the reduced-order model $\left(n_{k}=48\right)$. The control TEF deflections reach a steady-state value almost four revolutions. Figure 5.12 shows the control TEF deflections for all blades, obtained from the closed-loop GENUVP/GAST computer simulations. 

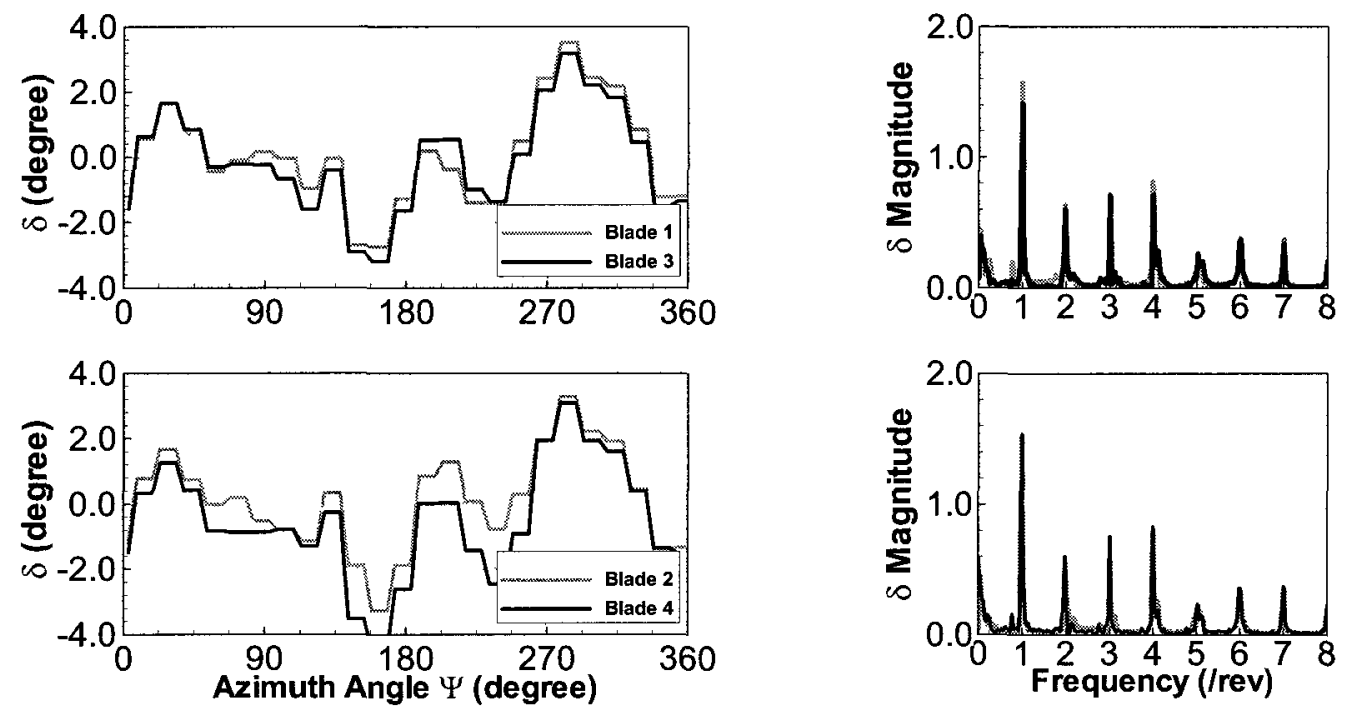

Figure 5.12: Control of 4/rev rotating frame blade load, $F_{z}$. Control command given to all TEFs $\delta_{1,2,3,4}$ by the $H_{2}$ controller.

The results of closed-loop simulations are summarized in Fig. 5.13 and 5.14. In Fig. 5.13, the frequency responses obtained from open-loop and closed-loop control simulations for the rotating frame vertical blade load, $F_{z, 1,2,3,4}$ for all blades are presented. 

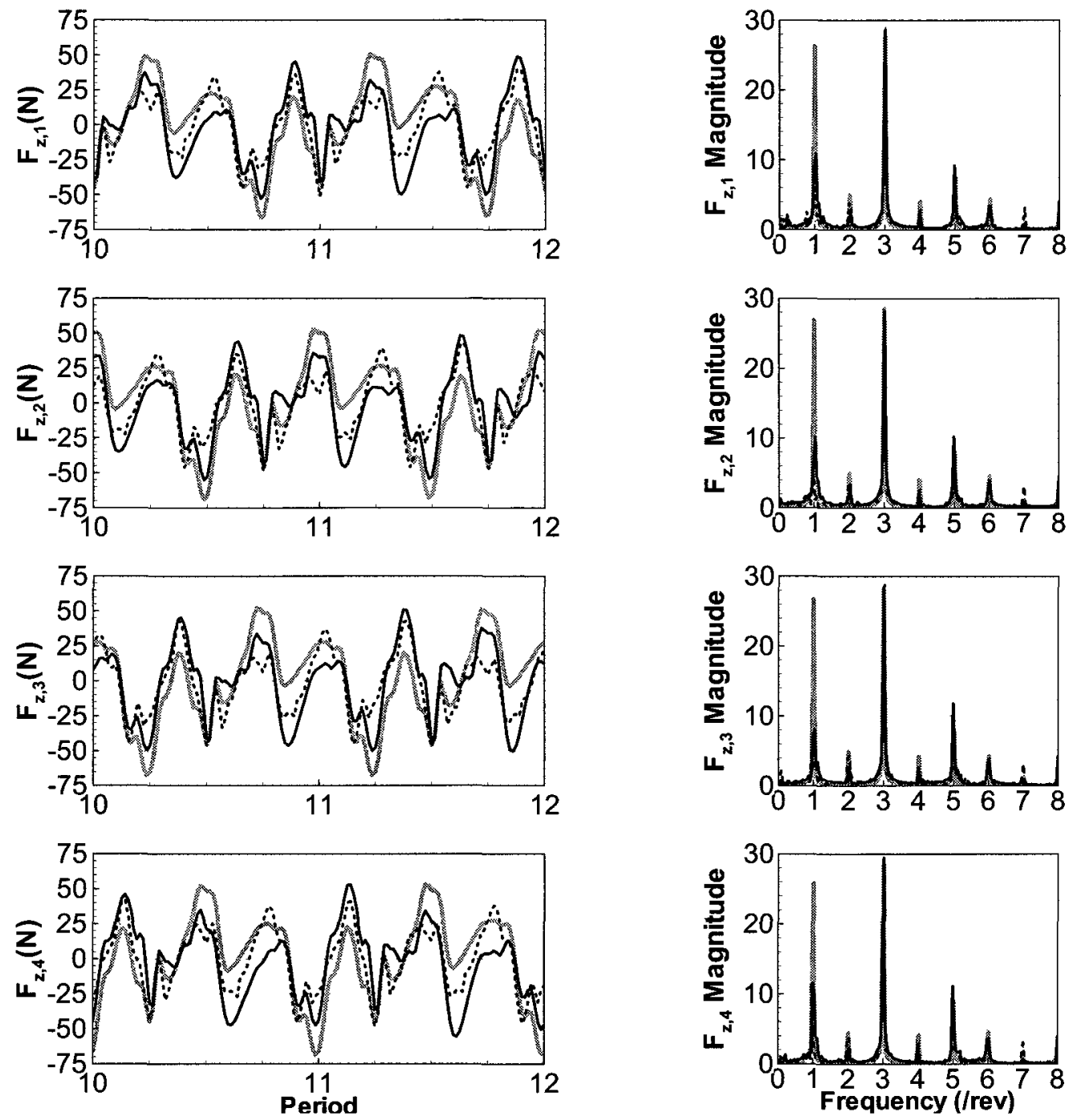

Figure 5.13: Control of 4/rev rotating frame blade load, $F_{z}$. Closed-loop control simulations: comparison between the baseline and controlled blade loads, $F_{z}$ obtained from the reduced-order model and the GENUVP/GAST simulations.

The rotating frame blade load, $F_{z}$ was suppressed at the $1 /$ rev, $2 /$ rev and $4 /$ rev fre- 
quencies by $59 \%, 52 \%$, and $30 \%$, respectively. While there was not any significant excitation recorded at $3 / r e v$, the amplitudes at frequencies $5 /$ rev and $8 / r e v$ were excited by $20 \%$ and $67 \%$, respectively. The amplitudes at $6 / \mathrm{rev}, 7 / \mathrm{rev}$ and $9 / \mathrm{rev}$ were reduced by $21 \%, 36 \%$ and $40 \%$, respectively. The rotating frame blade pitch moment, $M_{y}$ was also suppressed at the $1 /$ rev and $2 /$ rev frequencies by $31 \%$ and $36 \%$, respectively. The amplitude of vibrations at frequencies higher than $4 / r e v$ were also excited by approximately $20 \%$.
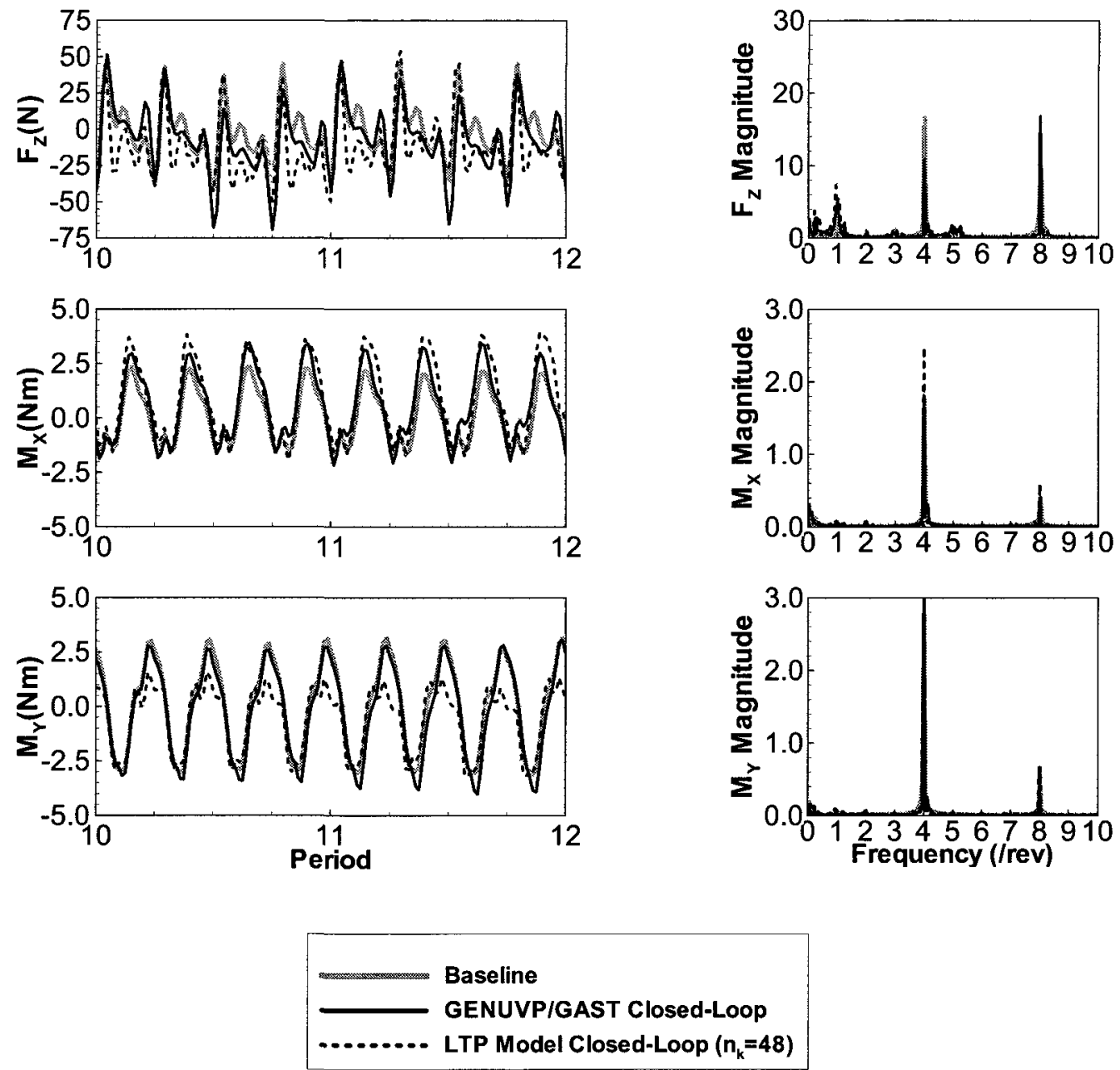

Figure 5.14: Control of 4/rev rotating frame blade load, $F_{z}$. Closed-loop control simulations: comparison between the baseline and the controlled hub loads, $F_{Z}, M_{X}$ and $M_{Y}$ obtained from the reduced-order model and the GENUVP/GAST simulations. 
The vertical hub load, $F_{Z}$ was suppressed at $4 /$ rev by $34 \%$. However, the higher harmonics, $8 / \mathrm{rev}$ and $12 / \mathrm{rev}$ were excited by $70 \%$ and $27 \%$, respectively. In addition, the resultant hub roll and pitch moments that were not taken into consideration in the controller design were adversely affected by the control action. While the amplitude of the hub roll moment, $M_{X}$ was not affected by the TEF control at $4 / r e v$, the hub pitch moment, $M_{Y}$ was excited by $23 \%$. Both hub moments were reduced at $8 /$ rev approximately $20 \%$ but excited at $12 /$ rev by about $50 \%$.

Imposing the same disturbance weight to the blades in the controller design (i.e. no phase shift) guarantees that the TEF deflections would reach the same steady-state value for all blades with respect to the blade azimuth location.

\subsubsection{All Blade Analysis: Suppression of the Rotating Frame Blade Pitch Moment, $M_{y}$ at $3 /$ rev}

The system model obtained from the all blade analysis $\left(n_{k}=16\right)$ in a four-blade configuration was used as a plant model in the feedback configuration given in Fig. 5.1. The performance weight for the measured output was selected to minimize the vibratory rotating frame blade load, $M_{y}$ especially at the $3 /$ rev frequency. The disturbance weight included the baseline disturbances in the frequency range $0-8 /$ rev with a phase difference. The selected weights are presented in Fig. 5.15. It was necessary to include the disturbances at higher frequencies to prevent the instabilities caused by the unmodelled disturbances. The resultant controller order was, $n_{K_{k}}=160$. 

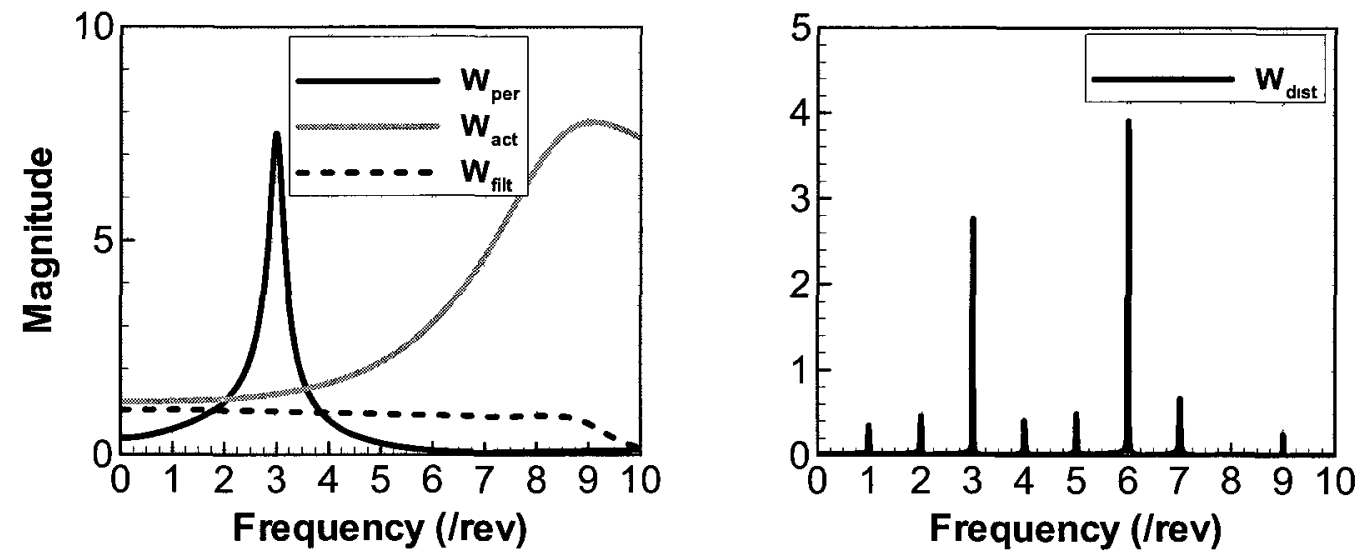

Figure 5.15: Control of 3/rev rotating frame blade load, $M_{y} . H_{2}$ controller design performance and disturbance weights.

The controller resulted in TEF deflections, obtained from the reduced-order model $\left(n_{k}=\right.$ 48) and the GENUVP/GAST aeroelastic simulations, are presented in Fig. 5.16. The steady-state TEF deflections were maintained by two revolutions in both simulations. 

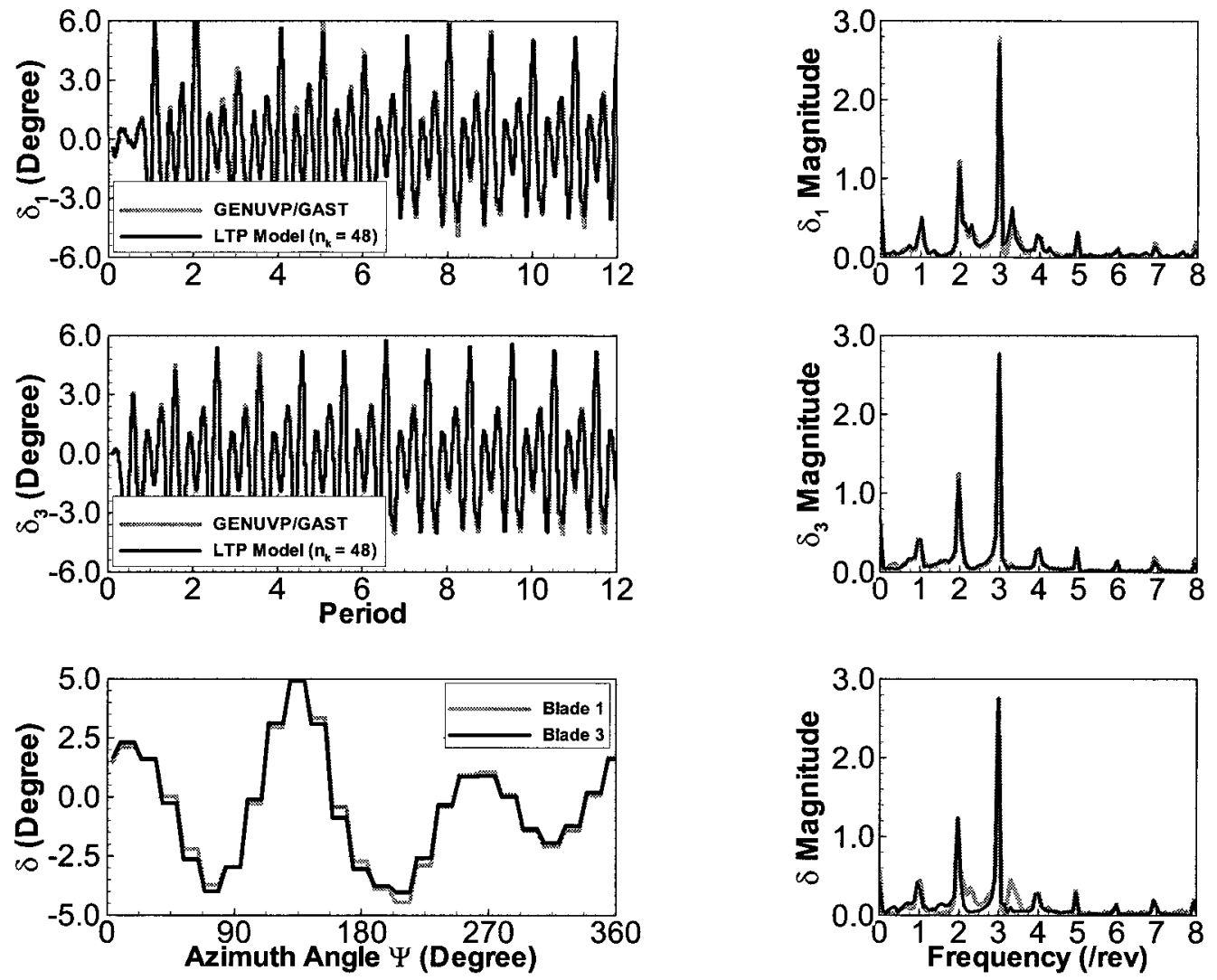

Figure 5.16: Control of 3/rev rotating frame blade load, $M_{y}$. Control command given to the TEF $\delta_{1}$ and $\delta_{3}$ by the $H_{2}$ controller.

The helicopter rotor system response in terms of rotating frame blade loads, $F_{z}$ and $M_{y}$ is presented in Fig. 5.17. Although the alleviation of the $3 / r e v$ frequency component of the blade pitch moment, $M_{y}$ was predicted very well by the reduced-order model, the reduction in the rotating frame blade load $F_{z}$ at $4 /$ rev frequency was not observed. The model predicted the amplitude of the vibration caused by the TEF deflections at $4 / r e v$ accurately, yet it was not capable of capturing the phase at $4 / r e v$. Further tuning of the LTP model would be necessary, if $3 /$ rev TEF deflections will be used to control the $4 / r e v$ rotating frame blade load, $F_{z}$. 

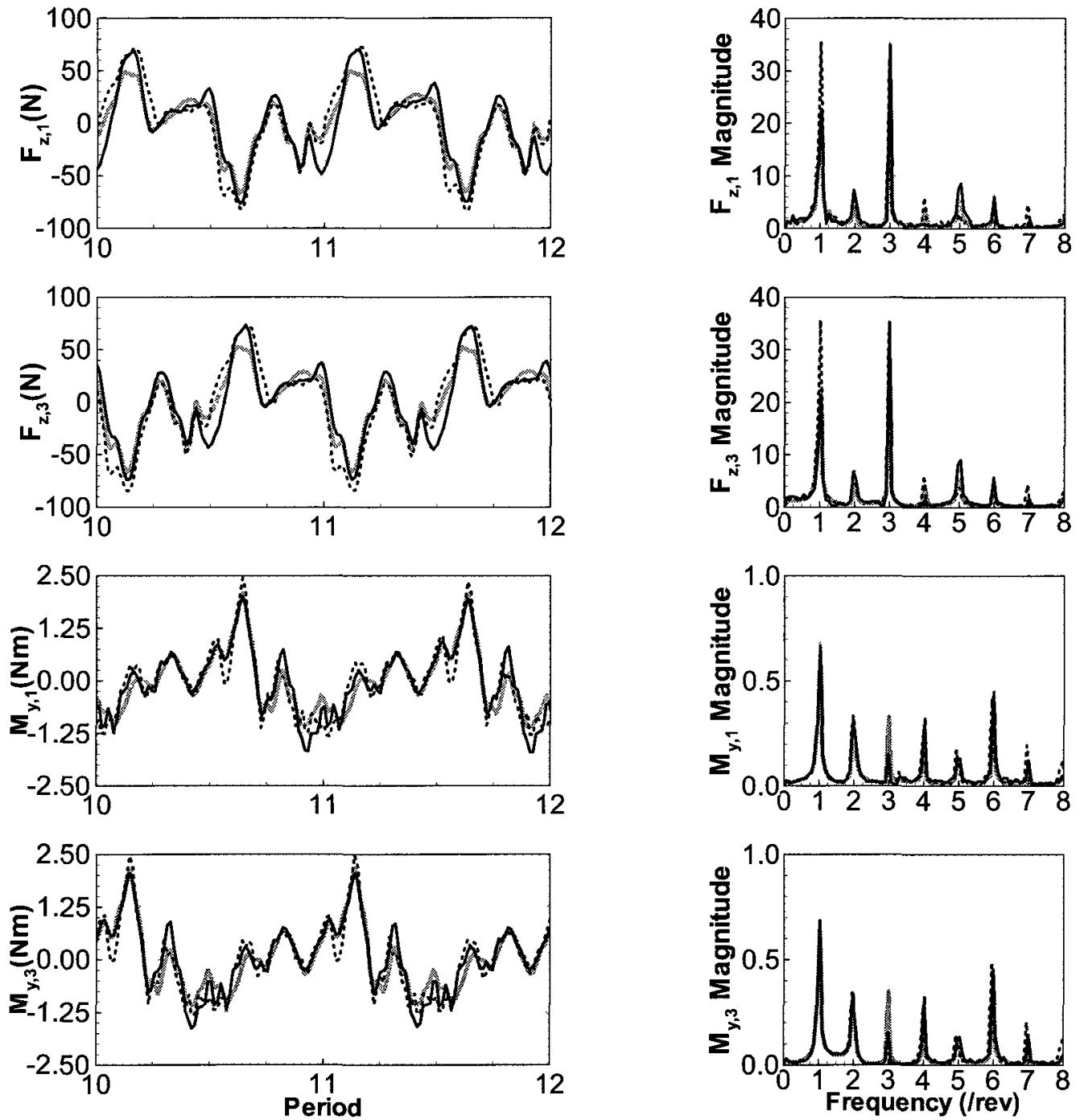

Figure 5.17: Control of 3/rev rotating frame blade load, $M_{y}$. Closed-loop control simulations: comparison between the baseline and the controlled blade loads, $F_{z}$ and $M_{y}$ obtained from the reduced-order model and the GENUVP/GAST simulations.

From the closed-loop controller simulations carried out using the GENUVP/GAST 
aeroelastic code, $57 \%$ amplitude reduction was obtained at the $3 / r e v$ frequency for $M_{y}$, as imposed by the performance specifications. The $M_{y}$ amplitude at the $2 / r e v$ and $4 / r e v$ frequencies was excited by $20 \%$ and $54 \%$, respectively. The effect of TEF on the other harmonics was not significant. As a result of $3 /$ rev control TEF deflections, the rotating frame blade load, $F_{z}$ was reduced by $70 \%$ at the $4 /$ rev frequency. However, the $F_{z}$ amplitude at the $2 / r e v, 3 / r e v, 5 / r e v$ frequencies were excited by $71 \%, 20 \%$ and $66 \%$, respectively.
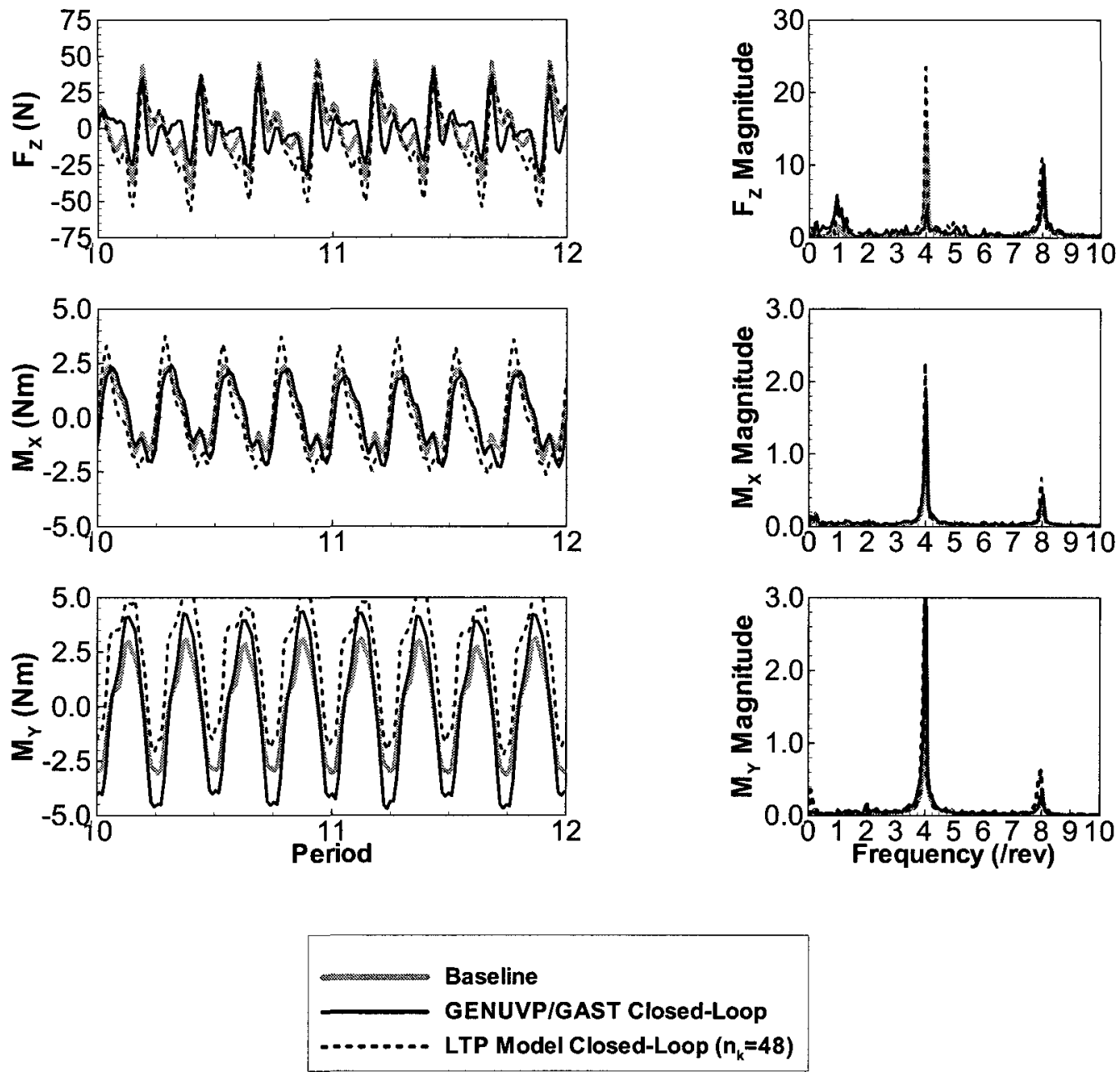

Figure 5.18: Control of 3/rev rotating frame blade load, $M_{y}$. Closed-loop control simulations: comparison between the baseline and controlled hub loads, $F_{Z}, M_{X}$ and $M_{Y}$ obtained from the reduced-order model and the GENUVP/GAST simulations. 
Steady-state values for $F_{Z}$ were obtained after four revolutions. The suppression in the amplitude of the rotating frame blade load, $F_{z}$ at the $4 /$ rev frequency resulted in $76 \%$ reduction in the vertical hub load, $F_{Z}$, and no higher harmonic excitation was recorded. The hub roll moment, $M_{X}$ was excited by less than $10 \%$ at both $4 /$ rev and $8 /$ rev, and the hub pitch moment, $M_{Y}$ was excited at $4 /$ rev by $44 \%$.

The system model that gives a map between the TEF deflection and the blade pitch moment, $M_{y}$ has very high direct feed-through term $D_{22, k}$. The regular $H_{2}$ controller synthesis problem imposes a zero direct feed-through term assumption and requires the reconstruction of the controller based on Eq. 3.40 (see Section 3.6.2) . Unfortunately, with the zero direct feed-through term assumption in the $\mathrm{H}_{2}$ controller synthesis, the ability of the TEF to change the blade pitch moment, $M_{y}$ becomes very limited. This caused numerical problems in the design. Several attempts to suppress the vibration at other frequencies in the frequency range $0-5 / r e v$ of $M_{y}$ failed. The use of singular $H_{2}$ controller design, which removes the assumption of zero $D_{22}$ term, should be explored in future work [131,132].

\subsection{Closed-Loop Control Results for Different Forward Flight Cases}

The system identification and controller design were carried out for the selected forwardflight case, with an advance ratio, $\mu=0.28$. The change in the system model with the advance ratio was omitted; parameter-varying system model identification was considered as a future work. In addition, in the controller design this change was not modeled as an uncertainty, which is a limitation in the $H_{2}$ controller synthesis method. Therefore, the $H_{2}$ controller could only work for the selected forward-flight case with the defined performance. The performance would degrade or instabilities could occur when the controller is used in a system away from that modeled system. To investigate the performance degradation of the $H_{2}$ controller, different forward flight cases were considered. The controller presented in Section 5.6.2 was tested for two different forward flight scenarios, with advance ratios, $\mu=0.224$ and $\mu=0.17$ that represents mid speed and low speed forward flight regimes, respectively. 


\subsubsection{Closed-Loop Control Results for Forward Flight Case with Advance Ratio, $\mu=0.224$}

In this forward flight case same wind tunnel trim conditions (see Section 2.3.2), were satisfied with the swash plate settings; collective pitch angle $\theta_{0}=5.56^{\circ}$, and cyclic pitch angles, $\theta_{1, c}=1.65^{\circ}, \theta_{1, s}=-3.03^{\circ}$. First, the helicopter rotor blade system reached the steadystate oscillatory trim condition, then the baseline vibratory rotating frame blade loads and hub loads were analyzed and compared to the $\mu=0.28$ forward-flight case. At this new baseline case, there were $63 \%$ and $27 \%$ reductions in the $1 /$ rev and $3 /$ rev frequencies of the rotating frame blade load, $F_{z, 1}$, respectively. However, there was a $67 \%$ increase in the $5 /$ rev frequency of $F_{z, 1}$. The $1 /$ rev, 2/rev and $6 /$ rev frequency contents of the rotating frame blade pitch moment, $M_{y, 1}$ were obtained $21 \%, 34 \%$, and $27 \%$ less than that recorded at $\mu=0.28$, respectively. The resultant vertical hub load, $F_{Z}$ had a $10 \%$ increase in $4 / r e v$, and a $35 \%$ decrease in the $8 /$ rev frequency content. The hub roll and pitch moments showed $7 \%$ and $22 \%$ decreases in the $4 / r e v$ frequency content. Based on these observations, we can conclude that the rotating frame baseline disturbances, which were modeled as output disturbance weight, $W_{\text {dist }}$ (see Fig. 5.11), differ for the two different forward-flight cases.

In addition, the system model that gives a map between the TEF deflection and the rotating frame blade loads $F_{z}$ and $M_{y}$ varies with the advance ratio. For example, for the same 3/rev TEF deflections applied to all blades, the net response of the helicopter rotor system in terms of the rotating frame blade load $F_{z, 1}$ to the 3/rev TEF deflections showed differences for the two different advance ratios. While the amplitudes at the $3 /$ rev frequency remained the same, the amplitudes at the $1 /$ rev and $5 /$ rev frequencies increased while at the $2 /$ rev and $4 / r e v$ frequencies they decreased. That is, while the transfer function $G_{0}$ did not change, both $G_{ \pm 1}$ and $G_{ \pm 2}$ were affected by the change in the velocity. Moreover, when the TEF is deflected at the $3 /$ rev frequency, the rotating frame blade load $F_{z}$ on the other blades were affected mostly at the $5 / r e v$ frequency. Hence, the system model that gives a map between the TEF deflection and the rotating frame blade loads $F_{z}$ and $M_{y}$ is no longer valid for different advance ratios. Therefore, performance degradation, or instabilities in the closed-loop system becomes unavoidable.

At this forward flight case, the controller presented in Section 5.6.2 was applied to 
reduce the $4 /$ rev frequency component of the rotating frame blade load, $F_{z}$. The closedloop control simulations were carried out using the GENUVP/GAST aeroelastic code and the resultant control TEF deflections are presented in Fig. 5.19 for blade one and three. After approximately six revolutions, the TEF deflections reached the steady-state value.
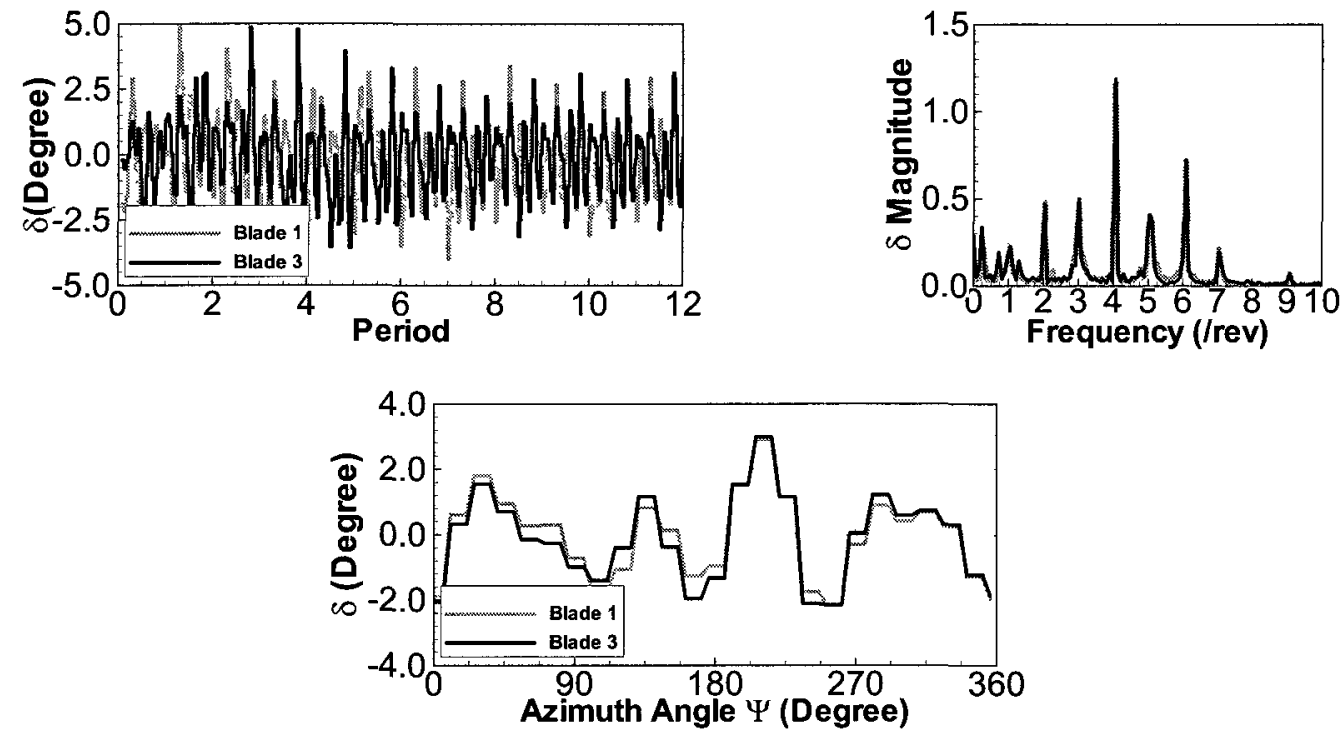

Figure 5.19: Control of 4/rev rotating frame blade load, $F_{z}$. Control command given to the TEF $\delta_{1}$ and $\delta_{3}$ by the $H_{2}$ controller, advance ratio, $\mu=0.224$.

The uncontrolled, and controlled helicopter rotor blade system responses obtained from the GENUVP/GAST aeroelastic simulations are compared in Fig. 5.20 for the rotating frame blade loads, $F_{z}$ and $M_{y}$ and in Fig. 5.21 for the hub loads. 

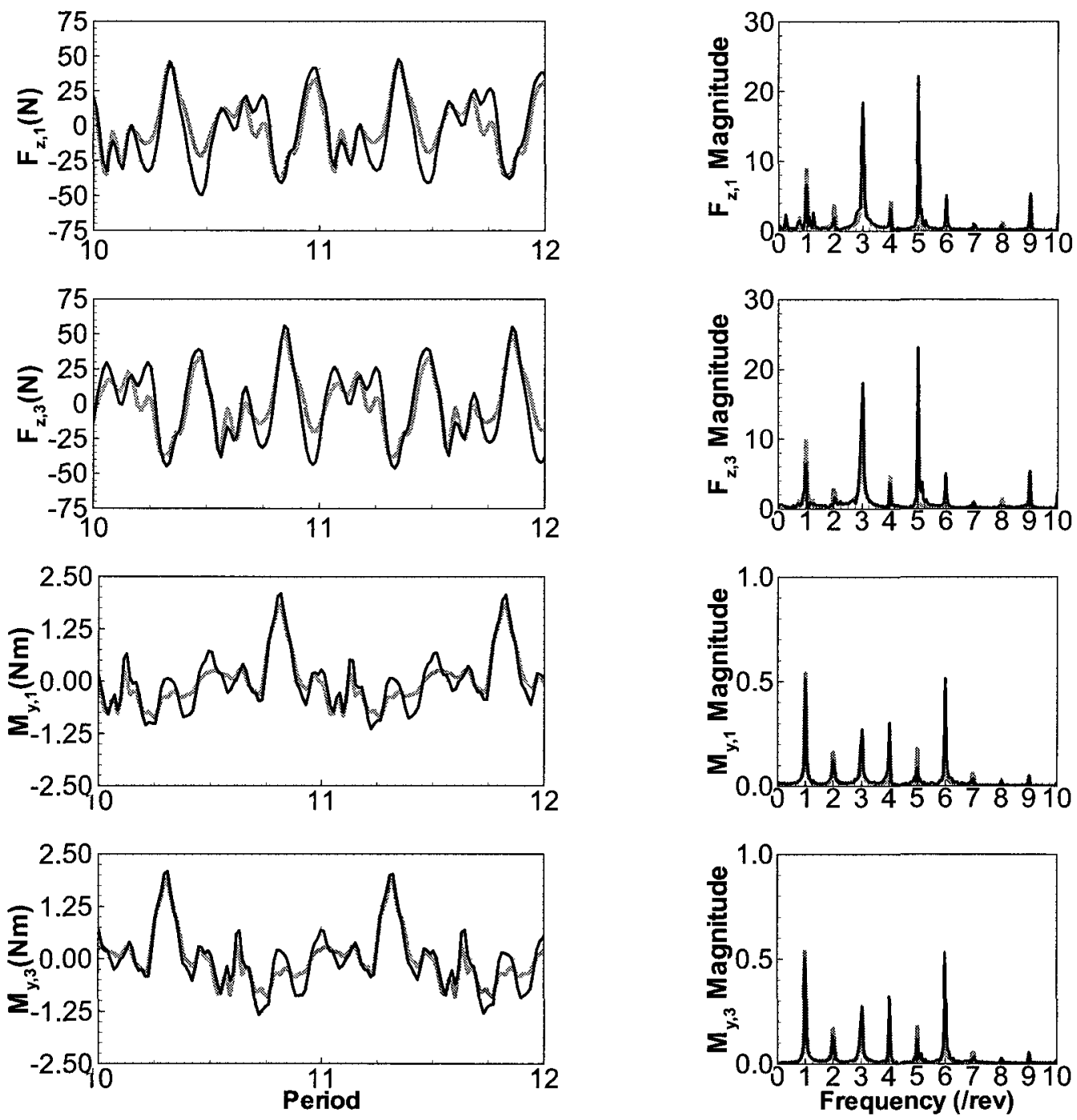

Figure 5.20: Control of 4/rev rotating frame blade load, $F_{z}$. Closed-loop control simulations: comparison between the baseline and controlled blade loads, $F_{z}$ obtained from the GENUVP/GAST simulations. at advance ratio, $\mu=0.224$.

The amplitude of the rotating frame blade load $F_{z}$ was reduced at the $1 /$ rev, $2 /$ rev and $4 / r e v$ frequencies approximately by $20 \%, 10 \%$ and $\% 10$, respectively. The performance of the controller was degraded almost $1 / 3$ as compared to the advance ratio, $\mu=0.28$ forwardflight case. The reduction in the rotating frame pitch moment, $M_{y}$ was not significant for lower frequencies, and at the $5 /$ rev frequency a $20 \%$ reduction was recorded. 

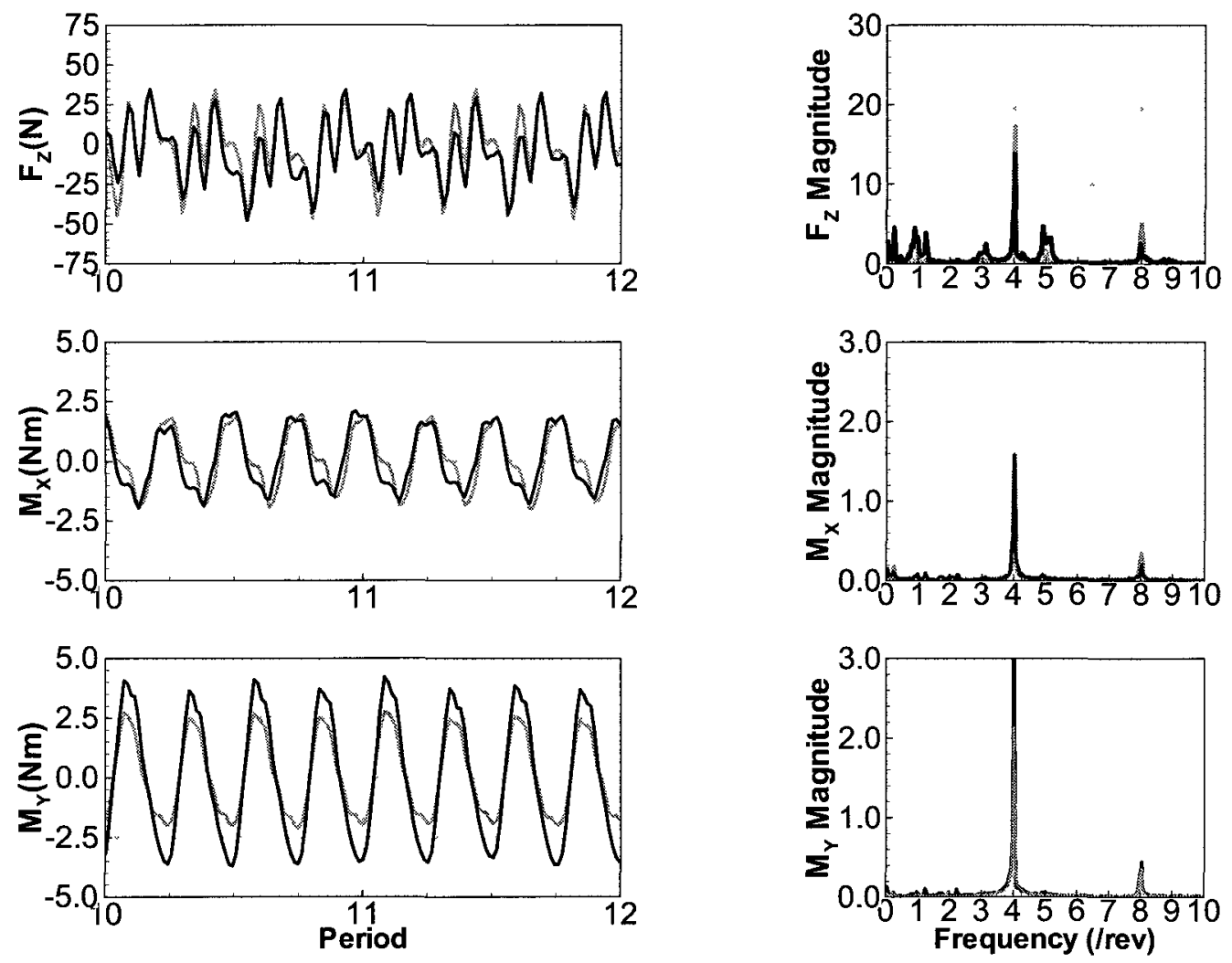

Figure 5.21: Control of 4/rev rotating frame blade load, $F_{z}$. Closed-loop control simulations: comparison between the baseline and controlled hub loads, $F_{Z}, M_{X}$ and $M_{Y}$ obtained from the GENUVP/GAST simulations, at advance ratio, $\mu=0.224$.

The resultant vertical hub load, $F_{Z}$ was reduced approximately by $20 \%$ for this forwardflight case. The same controller provided $34 \%$ suppression in the hub load, $F_{Z}$ for the forward-flight case where the controller design was carried out. While the amplitude of the hub roll moment, $M_{X}$ was not affected, the hub pitch moment was excited by $64 \%$. 


\subsubsection{Closed-Loop Control Results for Forward Flight Case with Advance} Ratio, $\mu=0.17$

At this forward flight case, the collective and cyclic swash plate angles that satisfy the wind tunnel trim conditions(see Section 2.3.2) were obtained as $\theta_{0}=5.96^{\circ}, \theta_{1, c}=2.02^{\circ}$, and $\theta_{1, s}=-2.32^{\circ}$. After the helicopter rotor blade system reached the steady-state trimmed forward-flight condition, the change in the frequency content of the rotating frame blade and hub loads were analyzed and compared to the forward-flight case with $\mu=0.28$. While both the $1 /$ rev and $3 /$ rev frequency content of $F_{z, 1}$ was recorded $70 \%$ less, the $5 /$ rev frequency content of $F_{z, 1}$ increased almost $70 \%$. In addition, the rotating frame blade pitch moment, $M_{y, 1}$ showed reduction in the $1 /$ rev, $2 /$ rev, $3 /$ rev frequency content, at amounts of $35 \%, 44 \%, 30 \%$, respectively. The amplitude at the $5 / r e v$ frequency increased almost $50 \%$. While the amplitude change in the $4 / r e v$ frequency content of the vertical hub load, $F_{Z}$ was negligible, the $8 /$ rev frequency content increased by $68 \%$. Both the hub roll moment, $M_{X}$ and hub pitch moment, $M_{Y}$ showed $35 \%$ decrease in the $4 /$ rev frequency content, whereas the amplitude of the $8 / \mathrm{rev}$ frequency content increased by $60 \%$ and $90 \%$, respectively. When the advance ratio was lowered, while the amplitudes of the baseline disturbances at the $1 /$ rev and $3 /$ rev frequencies decreased for the rotating frame blade loads, $F_{z}$ and $M_{y}$. The amplitudes of the same loads increased at the $5 / r e v$ frequency.

To suppress the $4 / r e v$ frequency component of the rotating frame blade load, $F_{z}$, the same controller presented in Section 5.6.2 was applied. The resultant TEF deflections is given in Fig. 5.22. The amplitude of the control TEF deflections become larger and it exceeded the imposed limit of $5^{\circ}$ peak-to-peak amplitude. 

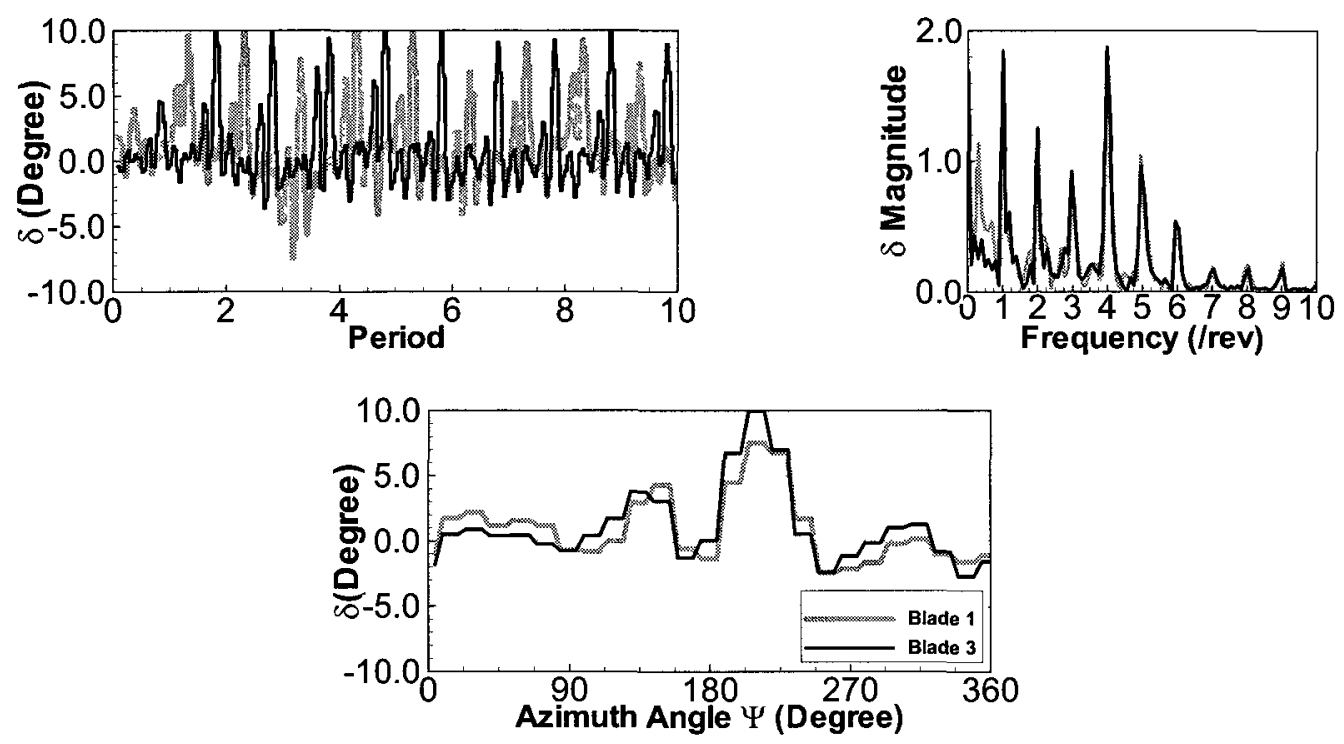

Figure 5.22: Control of $4 / r e v$ rotating frame blade load, $F_{z}$. Control command given to the TEF $\delta_{1}$ and $\delta_{3}$ by the $H_{2}$ controller, at advance ratio, $\mu=0.17$.

In Fig. 5.23, the helicopter rotor system response is presented in terms of rotating frame blade loads $F_{z, 1}$ and $M_{y, 1}$. Although, the helicopter rotor system remained stable, large $1 /$ rev and $5 /$ rev excitations were recorded in the $F_{z, 1}$. The amplitude of the rotating frame blade pitch moment, $M_{y}$ at the $4 / r e v$ and $6 / r e v$ frequencies was doubled. 

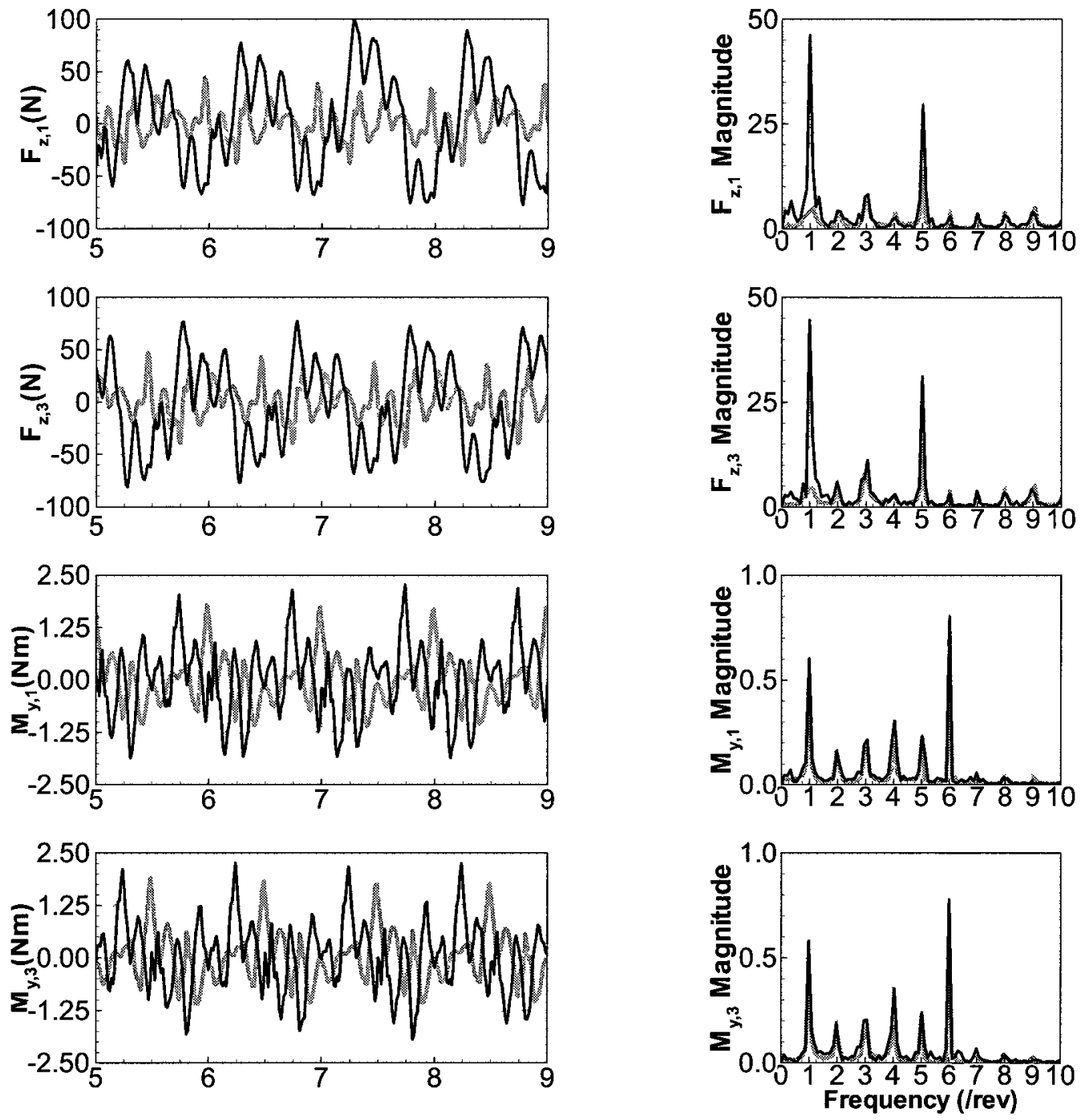

Baseline

GENUVP/GAST Closed-Loop

Figure 5.23: Control of 4/rev rotating frame blade load, $F_{z}$. Closed-loop control simulations: comparison between the baseline and controlled blade loads, $F_{z}$ obtained from the GENUVP/GAST simulations, at advance ratio, $\mu=0.17$. 

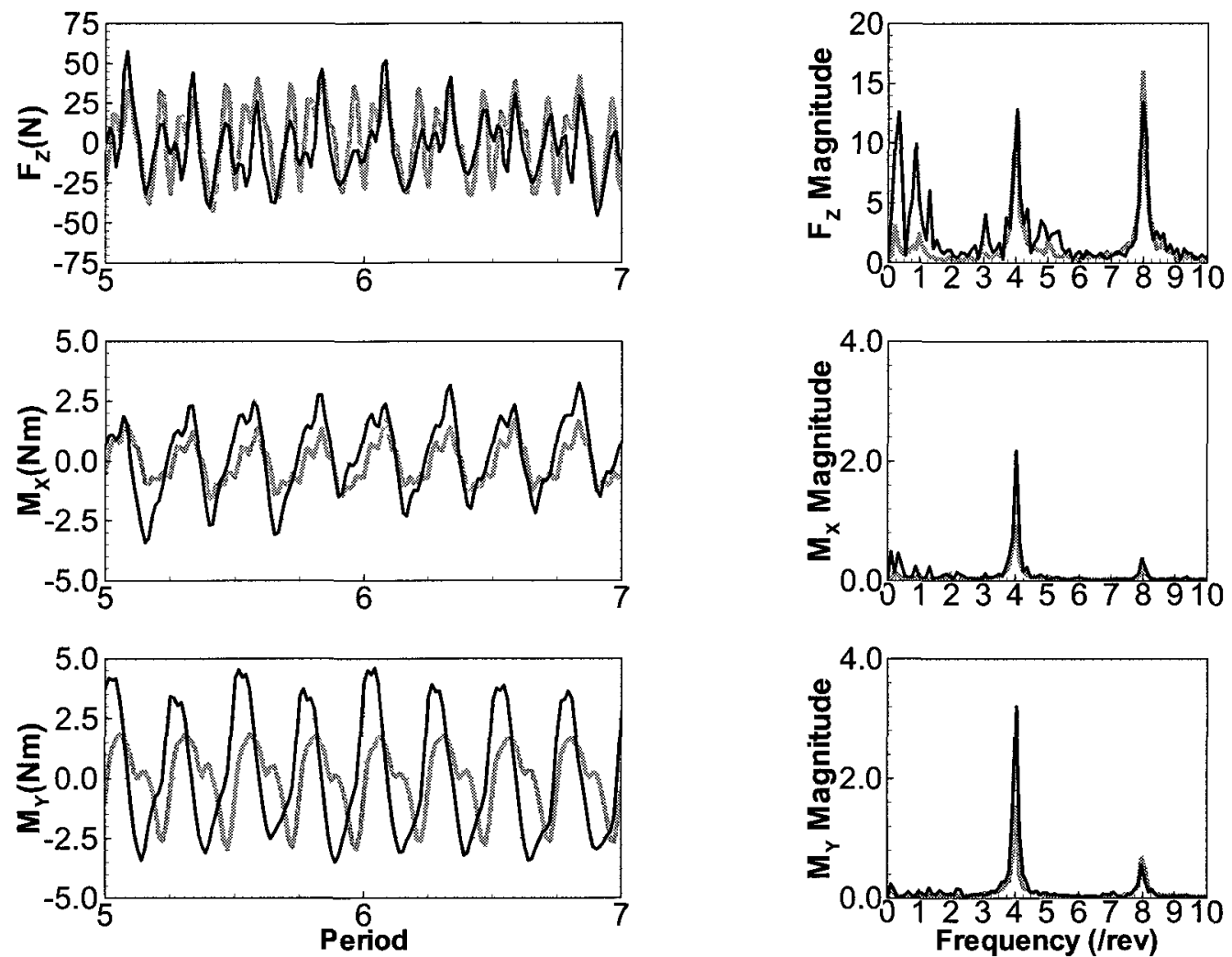

Baseline

GENUVP/GAST Closed-Loop

Figure 5.24: Control of 4/rev rotating frame blade load, $F_{z}$. Closed-loop control simulations: comparison between the baseline and controlled hub loads, $F_{Z}, M_{X}$ and $M_{Y}$ obtained from the GENUVP/GAST simulations, at advance ratio, $\mu=0.17$.

The changes in the rotating frame blade loads cancel out for the vertical hub load, $F_{Z}$, yet the low-frequency dynamics at $0.2 / r e v$ was excited. In addition, the aimed $4 / r e v$ reduction in $F_{Z}$ could not be attained. The amplitudes of the hub roll and hub pitch moments at the 4/rev frequencies were almost doubled by the control action. The closedloop control results presented for $\mu=0.224$ and $\mu=0.17$ showed that when the system moves away from the central model, based on which the controller design was carried out, the $\mathrm{H}_{2}$ controller cannot guarantee the same performance and stability characteristics of the original design. To overcome this, it is necessary to introduce a parameter varying 
state-space model, as given in Eq. 5.4.

$$
\left[\begin{array}{c}
x_{k+1} \\
y_{k}
\end{array}\right]=\left[\begin{array}{cc}
A_{k}\left(\mu_{\imath}\right) & B_{k}\left(\mu_{\imath}\right) \\
C_{k}\left(\mu_{\imath}\right) & D_{k}\left(\mu_{\imath}\right)
\end{array}\right]\left[\begin{array}{l}
x_{k} \\
u_{k}
\end{array}\right]
$$

where

$$
\begin{aligned}
\mu_{\imath}=0, \Delta \mu, \cdots \mu_{\max } & \\
A_{k+N}\left(\mu_{\imath}\right)=A_{k}\left(\mu_{\imath}\right) \in \mathbb{R}^{n_{k} \times n_{k}}, & B_{k+N}\left(\mu_{\imath}\right)=B_{k}\left(\mu_{\imath}\right) \in \mathbb{R}^{n_{k} \times m_{k}} \\
C_{k+N}\left(\mu_{\imath}\right)=C_{k}\left(\mu_{\imath}\right) \in \mathbb{R}^{p_{k} \times n_{k}}, & D_{k+N}\left(\mu_{\imath}\right)=D_{k}\left(\mu_{\imath}\right) \in \mathbb{R}^{p_{k} \times m_{k}}
\end{aligned}
$$

The system model is obtained for certain advance ratios. For the identification of the statespace matrices, the procedure given in Section 3.4 is repeated for each advance ratio. For the controller design, there are different options available. They are listed as follows:

- Time-periodic $H_{2}$ or $H_{\infty}$ controllers can be designed for each advance ratio and then a gain scheduling can be applied for the forward-flight speeds other than the selected ones. Controller switching will be required according to the advance ratio.

- Time-periodic robust $H_{2}$ or $H_{\infty}$ controllers can be designed modeling the parametric uncertainty in the system model caused by the change in the advance ratio. In this approach, gain scheduling will not be needed, the controller would perform the same between the advance ratios, $\mu \in\left[\mu_{\imath} \pm \Delta \mu_{\imath} / 2\right]$. Controller switching according to the advance ratio will be required. However, to solve this controller synthesis problem, solving two time-periodic Riccati Equations only will not be sufficient; extension of the available time-invariant controller synthesis methods to time-periodic systems is required. These methods are mainly based on an iterative solution between the controller synthesis and optimization of the scalings [88,133,134], and based on the LMI formulation $[52,99]$. Unfortunately, the computational effort could be very demanding, therefore, the computational cost evaluation should be performed before the design. 


\subsection{Conclusion}

In this Chapter, the implementation of LTP $H_{\infty}$ and $H_{2}$ (LQG) controller synthesis methods to suppress the helicopter rotor system vibration using TEFs is presented. The implementation problems associated with the synthesis of $H_{\infty}$ controllers using LMIs for this specific application are stated. The $\mathrm{H}_{2}$ controllers were designed by utilizing an in-house discretetime periodic Riccati solver. The controllers' performance was evaluated by closed-loop control simulations using both the reduced order models and the GENUVP/GAST fullorder aeroelastic code. These simulations provided similar results with few exceptions.

Time-periodic controller design based on LMI formulation was initially motivated by the availability of the software for optimization in Matlab. However, system model with high dimension, $n_{M_{k}}$ and long period, $N$ caused implementation problems for this specific application. For the same period of $N=24$, even though memory problems occurred while solving the synthesis problem with LMI for system order greater than fifty, the discrete-time periodic Riccati Equation solver resulted in a controller from less than ten minutes up to ten hours when the order of the system, $n_{M_{k}}$ varies from thirty to three hundred. This time could be lowered substantially by parallel computation, and this study left as a future work.

The cost in computation is not specific for time-periodic $H_{2}, H_{\infty}$ controller synthesis methods. In early study [14], the time required to solve for the constrained optimization problem to find optimal flap deflections in real-time applications using Matlab's function FMINCON was also found to be too costly. By using the resultant TEF deflections obtained from either time-periodic $H_{2}$ or $H_{\infty}$ controllers as an initial guess for the optimization problem, the required time could also be shortened. Iterative coupling of Riccati based solution and optimization methods to solve for robust time-periodic $H_{2}$ and $H_{\infty}$ controllers should be explored for helicopter vibration suppression, yet initial estimate on the trade off between the increase in computational cost and gain in terms of robustness should be performed.

Apart from the computational limitations, there were some drawbacks of designing $H_{2}$ (LQG) controllers for helicopter vibration suppression. First, the detailed model of output disturbance representing the baseline disturbances was required; higher frequencies 
were excited when the disturbance model was limited for certain frequency range. The immediate result of this detailed modeling is the increase in the controller order. Balanced order reduction could be performed to decrease the controller order. Second, the controller synthesis was limited for a specific advance ratio, therefore, the resultant controller was not robust to change in the system model when the advance ratio is changed. The computational tools, which solve the robust control synthesis problems, have to be extended for timeperiodic systems. Lowering the computational cost could still be a challenge.

By using both controller design methods, the vibration of rotating frame vertical blade load, $F_{z}$ at $1 /$ rev frequency was successfully alleviated. To suppress $89 \%$ of the $1 /$ rev vibrations, less than 4-degree peak-to-peak TEF deflections are required.

By using a $\mathrm{H}_{2}$ controller, both rotating frame blade loads and hub loads were suppressed at different frequencies. It was observed that the $4 / r e v$ vibrations at the hub vertical load $F_{Z}$ can be suppressed either by exciting TEFs mainly with the $3 / r e v$ frequency or with the $4 / r e v$ frequency. In fact, this is a direct effect of the system being time-periodic. Since, when the TEFs are deflected with a certain frequency, the resultant blade loads have not only the frequency of TEF excitations but also the harmonics of the rotor revolution caused by the $G_{ \pm n}$ transfer functions.

In Section 5.6.2 it was shown that if the performance weight is selected to provide suppression at $4 /$ rev of the rotating frame blade load, $F_{z}$, the controller results in mainly 4/rev TEF deflections (i.e. the $G_{0}$ effect is dominant). However, by imposing the performance criteria to suppress the $3 /$ rev rotating frame blade pitch moment, $M_{y}$ the resultant controller provides mainly 3/rev TEF deflections, which eventually suppresses the 4/rev vertical hub load, $F_{Z}$. The use of $3 /$ rev TEF excitations to suppress the $4 /$ rev frequency content of $F_{z}$ requires accurate modeling of $G_{-1}$ both in amplitude and phase. The model obtained from the all blade analysis with order, $n_{k}=48$ however, fail to represent the phase shift at $4 / r e v$ caused by the $3 /$ rev TEF deflections. Therefore, the reduction at the $4 / r e v$ frequency component could not be predicted in the reduced-order model simulations.

It should be noted that, to suppress the $4 /$ rev vertical hub load, $F_{Z}$ it is not necessary to suppress the rotating frame blade pitch moment, $M_{y}$ at the $3 / r e v$ frequency. This is concluded as result of several trial simulations; imposing $3 /$ rev TEF deflections of different 
phases, without aiming suppression of $M_{y}$ at the $3 /$ rev frequency. It was observed that when the TEFs were deflected at the $3 /$ rev frequency having a certain phase, even though the blade pitch moment, $M_{y}$ was excited at $3 / r e v$, suppression of $F_{z}$ at the $4 / r e v$ frequency could still be attained.

The four-blade analysis was performed by imposing the disturbance weight being the same for all blades or with a phase shift between the blades. By this way, the possibility of canceling out the $4 / r e v$ component of the blade loads while transferring them to the non-rotating frame instead of suppressing them in the rotating frame was explored. When the same disturbance weight was used, the resultant augmented system in LFT form had the frequency content of $1-5 / \mathrm{rev}$. When the phase shift was imposed in the disturbance weights, the augmented system had mainly $4 /$ rev frequency content. After scaling the performance criteria at $4 / r e v$ frequency of $F_{Z}$, both approaches give the similar TEF control effect; suppressing the $4 / r e v$ frequency content of $F_{z}$ in the rotating frame.

The controller designed based on the system model obtained at the forward-flight case with advance ratio $\mu=0.28$ was tested for two different forward flight cases with advance ratios $\mu=0.224$ and $\mu=0.17$. The closed-loop simulations showed that the performance of the controller degrades and excessive excitations occur when the system model deviates from the original model. While the controller gives $34 \%$ reduction in the $4 /$ rev frequency content of $F_{Z}$ for $\mu=0.28$, at advance ratio $\mu=0.224$ this reduction goes down to $20 \%$. In addition, excessive excitations were observed at the $1 /$ rev ad $5 /$ rev frequency contents of the rotating frame blade load $F_{z}$ when the forward-flight speed was decreased. The advance ratio parameter dependent system identification was not performed and it is suggested as a future work. 


\section{Chapter 6}

\section{Conclusions and Recommendations}

In forward flight, helicopter rotor blades function within a highly complicated aerodynamic environment including both near-blade and far-blade aerodynamic phenomena, such as transonic flow with shock waves, dynamic stall and blade vortex interaction. These aerodynamic phenomena cause fluctuating aerodynamic loads on the rotor blades. These loads when coupled with the dynamic characteristics and elastic motion of the blade create excessive amount of noise and vibration. To reduce the helicopter vibration recent studies developed several active vibration control strategies. These control strategies aim at suppressing the vibration either after it is transferred to the frame or at its source by applying the control actuation using active pitch link, actively controlled flap, actively twisting the rotor blade or higher harmonics of swash plate. In active control, the control actuation is updated by the controller. To design a controller mathematically tractable, reduced-order

models are required. The models have to provide accurate prediction of the complex features of the helicopter aeroelasticity that are obtained from high-fidelity computer simulations or real-time experiments. In all previous studies, the reduced-order aeroelastic models were obtained by coupling independently reduced aerodynamic and structural dynamic models. These models then were used to design a controller. However, these controllers when implemented in high-fidelity computer simulations or in real-time experiments could not guarantee the stability and/or satisfy the performance criteria. 


\subsection{Conclusions}

In this thesis, a novel approach that provides accurate time-periodic models and timeperiodic $H_{2}$ and $H_{\infty}$ controllers that satisfy the stability and design performance criteria was analyzed. The approach was validated by designing controllers to suppress the helicopter rotor system vibration using actively controlled Trailing Edge Flaps (TEFs). The three main conclusions are summarized in the following sections.

\subsubsection{Important Aeroelastic Features of Helicopters Can Only be Cap- tured With High-Fidelity Coupled Aeroelastic Analysis}

Traditionally, both the aerodynamic and structural dynamics of the helicopter rotor systems were independently reduced to simplified models and then coupled to perform the aeroelastic analysis. These models are attractive owing that they are more general, i.e. applicable for wide range of flight scenarios, and they are ready to be used for controller design. These models provide a good preliminary knowledge on the helicopter rotor system response; however, they are not accurate enough to capture the complex features of coupled aeroelastic motion. Even though the controllers designed based on these reduced-order coupled models were proven to suppress the vibration satisfactorily, they were necessarily re-designed to satisfy the stability and performance criteria in high-fidelity full-order aeroelastic analysis or in real-time experiments.

The necessity of performing full-order coupled aeroelastic analysis was proven by computer simulations carried out with a Computational Fluid Dynamics (CFD)/ Computational Structural Dynamics (CSD) aeroelastic solver, the GENeral Unsteady Vortex Particle (GENUVP)/General Aerodynamic and Structural Prediction Tool (GAST) for our validation case. It was shown that the TEF deflection changes the rotating frame blade loads of not only the blade that the TEF is attached but also other blades. This influence is caused by the disturbed wake and could not be captured using traditional models, which either isolate the blade or ignore the wake disturbance associated with TEF deflection. In fact, isolated 2-D airfoil analysis with predefined wake models are commonly used in the traditional reduced-order models. 
Therefore, before order reduction, our results suggest that a coupled high-fidelity and full-order aeroelastic analysis is required to capture the important features of the helicopter aeroelasticity. The reduced-order models obtained from such coupled analysis are significantly more accurate and reliable. Yet, it is, of course, a trade off between using more general off-the-shelf reduced-order models or more accurate models of the specific system under consideration. Obtaining accurate models requires additional step in the analysis, system identification, and it is computationally demanding.

\subsubsection{Time-Periodic Models are Necessary to Obtain an Accurate Map Between Control Actuation and Helicopter Aeroelastic Response}

Traditionally, reduced-order models of the helicopters aerodynamic and structural response were assumed time-invariant. This seems advantageous since controller design for timeinvariant models are well developed and many computational tools are available. Unfortunately, time-invariant models are not accurate in predicting the actual helicopter rotor system response in forward flight. In Chapter 4 , the necessity of identifying time-periodic system models was demonstrated. For example, the relative percentage error norm $L_{2}$ was reduced from $70 \%$ to $25 \%$ for the rotating frame vertical blade load and from $70 \%$ to $51 \%$ for the rotating frame blade pitch moment, when the system was modeled as time-periodic (see Table 4.2). The identification was carried out using the Subspace Identification Method (SIM), which yields linear time-periodic system model in discrete-time state-space.

However, besides providing time-periodic models of the helicopter aeroelastic system, the SIM methodology was demonstrated to offer significant additional advantages over previous identification methods. Most importantly, the SIM method does not require a priori model knowledge. In the context of helicopter aeroelastic identification, a method such as SIM is more powerful as compared to parameter-based system identification methods. Firstly, the SIM method reduces the required number of revolutions to accurately identify the system model. The necessary number of revolutions to identify the system model was verified to be less than those required by the parameter-based system identification method. Secondly, from the characteristics of time-periodic impulse responses, it was concluded that the model set design in parameter-based system identification, if not done properly, could lead to high 
prediction errors and so increase the required time to estimate the system parameters. In fact, the blade loads to impulsive TEF deflections die out in three revolutions when the TEFs are deflected in the advancing side but they die out in less than a revolution when the TEFs are deflected in the retreading side (see Fig. 4.8). Hence the number parameters to be identified at each time step varies. This is an a priori knowledge that is necessary for accurate modeling using parameter-based system identification method, which is not required by the SIM. In addition, the SIM method offers the flexibility of using ensemble data obtained from several independent aeroelastic simulations. By simulating short period aeroelastic responses and ensembling them, the computational efficiency of the identification simulations was significantly increased.

Finally, having the state-space matrices at every time step in one revolution enables us to design time-periodic controllers that can update the control actuation at every time step. This improvement eliminates the numerical and implementation drawbacks of the time-lifting approach, where the control actuation is only updated at every period.

\subsubsection{Time-Periodic $H_{2}$ and $H_{\infty}$ Controllers Satisfy the Stability and Design Performance Criteria When Implemented in High-Fidelity Aeroelastic Analysis}

Time-periodic controller design is necessary to satisfy the closed-loop stability and performance in high-fidelity full-order aeroelastic simulations or in real-time implementations. The proposed approach suggests designing discrete-time periodic controllers in the $H_{\infty}$ and $\mathrm{H}_{2}$ framework by using two solution methods, namely; Linear Matrix Inequalities (LMIs) and solution of discrete-time periodic Riccati Equations. The LMI based formulation was motivated by the fact that no additional software is needed; i.e. MATLAB routines are available. However, for long-period high-dimension system models, the solution of the discrete-time periodic Riccati Equations are necessary. To accomplish this, an in-house periodic Riccati solver was developed.

In previous studies, the control actuation was updated every rotor revolution, which was followed by waiting one-fourth of a revolution for a four-bladed rotor to three revolutions so that the transient response dies out and the wake stabilizes. This waiting time was 
required to reach the quasi-steady condition to perform the harmonic analysis. In one of the previous studies, the effect of this waiting time in the system stability was analyzed, and it was shown that it can cause instabilities in the closed-loop system. In this study, the control actuation was updated at every time step, instead of at every rotor revolution, and this control update was performed continuously for the next revolutions.

In Chapter 5, the last step of the proposed approach was evaluated by designing timeperiodic $\mathrm{H}_{\infty}$ and $\mathrm{H}_{2}$ controllers and analyzing the closed-loop control results for helicopter vibration suppression using TEFs. Most of the closed-loop control simulations obtained from the reduced-order models and full-order high-fidelity aeroelastic simulations provided similar TEF deflections with few exceptions, indicating the accuracy of the reduced-order models. The TEF deflections reached steady-state values after a couple of revolutions, updating the control actuation at every time step. Vibration suppression of both the rotating frame blade loads and hub loads were attained without causing detrimental changes in the trim state values. It was observed that the disturbance modeling had to be performed carefully in the $\mathrm{H}_{2}$ controller design since unmodeled higher harmonics created instabilities in several simulations. Since the controllers were designed based on the system model at one fixed forward flight speed, and the change in the system model with the advance ratio was not modeled, robustness problems were demonstrated for different advance ratios.

\subsection{Recommendations}

We can categorize our recommendations considering on both the numerical and analytical aspects of the problem and their applicability for real-time experiments.

\subsubsection{Numerical-Analytical Improvements}

Future research should focus on obtaining system models applicable to broader flight scenarios and efficiently designing real-time implementable controllers that satisfy the robust stability and performance criteria. For this purpose:

- The Periodic Riccati Solver should be modified to design $H_{\infty}$ controllers.

- The stability characteristics of the closed-loop system should be analyzed in detail 
in comparison to the previous controller implementations. Previous implementations suspend the control actuation for a couple of revolutions to calculate the new control actuation to be applied in the next revolution.

- Different forward-flight conditions should be analyzed and parametrization of the discrete-time linear time-periodic state-space matrices with the advance ratio should be performed. Controllers should be designed for these time-periodic and parameter varying systems.

- Robust $H_{2}$ and $H_{\infty}$ controller designs should be performed including the uncertainties in the plant and its environment. Future research should focus on the modeling of ignored higher-order dynamics and uncertainties in the plant parameters to increase robustness $[52,88,99]$. Computationally efficient tools for the robust controller design of time-periodic systems should be developed.

- The combined solution of the Riccati-based controller design and the optimization should be explored to decrease the computational time necessary to design parameterbased $\left(K_{k}(\mu)\right)$ and robust controllers $[133,136]$.

\subsubsection{Modifications for Real-time Experiments}

To apply the proposed approach in real-time experiments, several modifications should be performed. Since, all of our analyses were based on the availability of the baseline vibrations, the modifications should involve the on-line identification of the baseline vibrations. For this purpose:

- The identification algorithm should be modified to provide on-line identification [137].

- Baseline vibrations should be identified for given trim settings. The resultant transfer function from this identification would provide the rotating frame blade loads for a given shaft angle, swash plate setting and advance ratio. The identification could be carried out by using the same algorithm or different approaches can be considered [123, $138-140]$. 
- The controllers should be designed off-line. Controller order should be reduced by periodic-balanced or balanced-free model order-reduction methods [126]. Finally, the performance of the controllers should be validated with wind tunnel experiments. 


\section{Appendix A}

\section{Background of LMIs and Block Diagonal Representation}

\section{A.1 Linear Matrix Inequality}

Linear Matrix Inequalities (LMIs) are used in formulation of convex optimization problems in system and control theory for stability analysis and controller design. As an illustrative example, formulation of the stability condition for time-periodic systems in terms of LMIs is presented in this section. Lyapunov's stability condition for time-periodic systems requires the existence of periodic positive-definite matrices, $P_{k}(x)$ for $k=1, \cdots, N$, $P_{1}(x)>0, P_{2}(x)>0 \ldots . P_{N}(x)>0$ that satisfy the stability condition given in Equation 3.23 . The periodic positive-definite matrices, $P_{k}$ can be written in terms of the variable vector, $x_{k} \in \mathbb{R}^{m}$, and known matrices, $P_{k_{\imath}}=P_{k_{\imath}}^{\prime} \in \mathbb{R}^{n} \times n, i=0, \cdots, m$, where $m=\frac{n(n+1)}{2}$ as:

$$
P_{k}(x)=P_{k_{0}}+\sum_{i=1}^{m} x_{k_{\imath}} P_{k_{\imath}}>0
$$

By using Equation A.1, Lyapunov's stability condition can be formulated as convex optimization problem, where the existence of a feasible set $x$ satisfying the stability condition 
is searched (see Equation A.2).

$$
\begin{array}{ll}
\text { feasibility } & x \\
\text { subject to } & A_{k}^{\prime}\left[P_{k+1_{0}}+\sum_{\imath=1}^{m} x_{k_{\imath}} P_{k+1_{\imath}}\right] A_{k}-\left[P_{k_{0}}+\sum_{\imath=1}^{m} x_{k_{\imath}} P_{k_{\imath}}\right]<0 \\
& \forall k=1, \cdots, N, \text { and } \\
& P_{k+N}=P_{k}
\end{array}
$$

for example for $A_{k} \in \mathbb{R}^{2 \times 2}$, the positive-definite $P_{k}(x)$ given in Equation A.1 can be written by defining the variable vector $x_{k}=\left[x_{k_{1}}, x_{k_{2}}, x_{k_{3}}\right]$ and with known matrices $P_{k_{\imath}}$

$$
\begin{aligned}
& P_{k}(x)=\left[\begin{array}{ll}
x_{k_{1}} & x_{k_{2}} \\
x_{k_{2}} & x_{k_{3}}
\end{array}\right] \\
& P_{k_{0}}=\left[\begin{array}{ll}
0 & 0 \\
0 & 0
\end{array}\right], P_{k_{1}}=\left[\begin{array}{ll}
1 & 0 \\
0 & 0
\end{array}\right], P_{k_{2}}=\left[\begin{array}{ll}
0 & 1 \\
1 & 0
\end{array}\right], \quad P_{k_{3}}=\left[\begin{array}{ll}
0 & 0 \\
0 & 1
\end{array}\right]
\end{aligned}
$$

The constraints given in the optimization problem are system of LMIs that can be expressed as a single LMI in the form:

$$
P(x)=\left[\begin{array}{cccc}
P_{1}(x) & 0 & \cdots & 0 \\
0 & P_{2}(x) & & 0 \\
\vdots & & \ddots & \vdots \\
0 & 0 & \cdots & P_{N}(x)
\end{array}\right]
$$

Once the convex optimization problem is formulated to search over either a feasible set $x_{i}$ or a minimization of a function based on $x_{i}$ with the LMIs being the constraints, Matlab's Optimization Toolbox can be used to solve the problem. If there is not any set of $x$ satisfying the stability condition, i.e. infeasible, the system is said to be unstable.

\section{A.2 Block-Diagonal Operator}

Block-diagonal operators are used to represent the LTP systems in cyclic formulation [77] and to formulate $H_{\infty}$ synthesis problem for time-varying systems [141]. A partitioned 
matrix, $M_{k}$ is given in Equation A.4, which was previously used to represent the statespace model (see Equation 3.24a in Section 3.6).

$$
M_{k}=\left[\begin{array}{cc}
F_{k} & G_{k} \\
R_{k} & S_{k}
\end{array}\right]: l_{2}^{m_{1}} \times l_{2}^{m_{2}} \rightarrow l_{2}^{p_{1}} \times \mathbb{l}_{2}^{p_{2}}
$$

the diagonal realization of $M_{k}$ is represented as $\llbracket M_{k} \rrbracket$ and given as [141]:

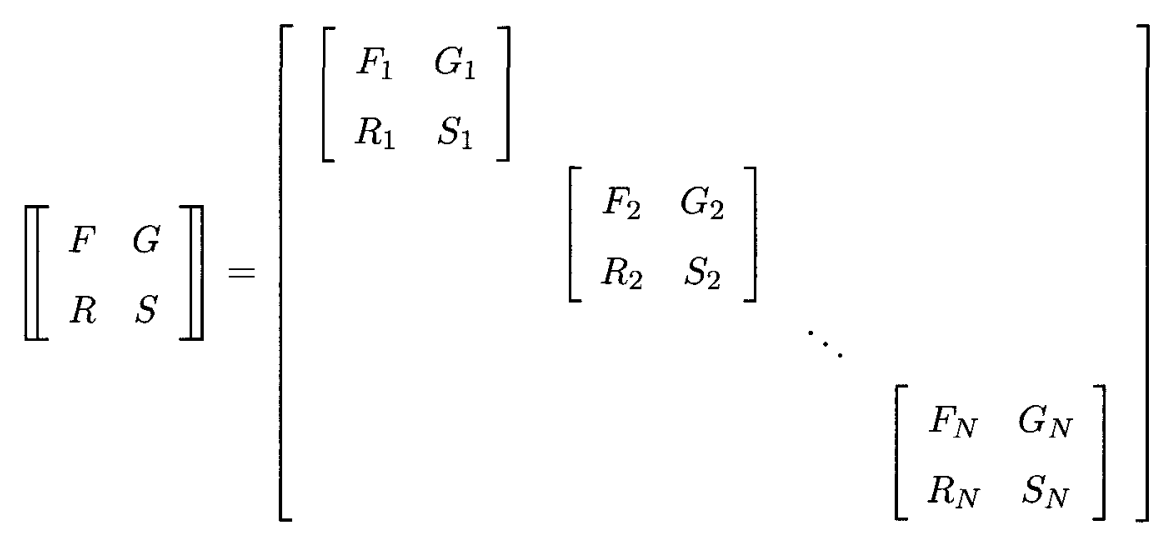

where

$$
\llbracket \begin{array}{cc}
F & G \\
R & S
\end{array} \rrbracket_{k}=\left[\begin{array}{cc}
F_{k} & G_{k} \\
R_{k} & S_{k}
\end{array}\right]
$$

The properties of this operator and its use in systems theory can be found in [141]. 


\section{Bibliography}

[1] J. Leishman, Principles of Helicopter Aerodynamics. Cambridge Aerospace Series, 2001.

[2] P. P. Friedmann and F. Bagnoud, "Rotary-wing aeroelasticity - current status and future trends," AIAA Journal, vol. 42, October 2004.

[3] W. Johnson, Helicopter Theory. Dover Publications, 1994.

[4] T. M. Peretz P. Friedmann, "Vibration reduction in rotorcraft using active control: a comparison of various approaches," Journal of Guidance, Control, And Dynamics, vol. 18 , no. 4, pp. $664-673,1995$.

[5] R. Cribbs and P. Friedmann, "Vibration reduction in rotorcraft using an enhanced ACSR model," in $41^{\text {st }}$ AIAA/ASME/ASCE/AHS Structures, Structural Dynamics and Materials Conference, (Atlanta, GA), 2000.

[6] Y. Chen, V. Wickramasinghe, and D. Zimcik, "Development of adaptive seat mounts for helicopter aircrew body vibration reduction," Journal of Vibration and Control, vol. 15 , no. 12 , pp. $1809-1825,2009$.

[7] P. Konstanzer, B. Enenk, P. Aubourg, and P. Cranga, "Recent advances in Eurocopters passive and active vibration control," in $64^{\text {th }}$ Anual Forum of American Helicopter Society International, (Montreal, Canada), 2008.

[8] M. Lovera, P. Colaneri, C. Malpica, and R. Celi, "Closed-loop aeromechanical stability analysis of HHC and IBC, with application to a hingless rotor helicopter," in $29^{\text {th }}$ European Rotorcraft Forum, (Friedrichshafen, Germany), September 2003. 
[9] C. Malpica, Contributıons to the dynamıcs of helıcopters with active rotor controls. PhD thesis, University of Maryland, College Park, 2008.

[10] A. Mander, D. Feszty. and F. Nitzsche, "Active pitch link actuator for impedance control of helicopter vibration," $64^{\text {th }}$ Annual Forum of the American Helicopter Socnety, 2008.

[11] M. Voskuijl, D. Walker, B. Manimala, and R. Kureemun, "First steps towards the design of an active pitch link loads reduction system using novel control techniques," in $31^{\text {st }}$ European Rotorcraft Forum, (Florence, Italy), September 2005.

[12] S. John, N. Wereley, and J. Sirohi, "Development of a piezohydraulic active pitch link for a swashplateless helicopter rotor," Journal of Atrcraft, vol. 46, no. 1, 2009.

[13] P. Friedmann, M. Terlizzie, and T. Myrtle, "New developments in vibration reduction with actively controlled trailing-edge flap," Mathematıcal and Computer Modelling, vol. 33, pp. 1055-1083, 2001.

[14] B. Roget, Individual blade control for vibration reduction of a helicopter with dissimilar blades. PhD thesis, University of Maryland, 2004.

[15] S. Hwang, Frequency domain system ıdentıfication of helıcopter rotor dynamics ıncorprating models with time perıodic coefficients. $\mathrm{PhD}$ thesis, University of Maryland at College Park, 1997.

[16] F. Nitzsche, D. Feszty, K. Khomutov, B. Lynch, A. Mander, and F. Ulker, "Design and instrumentation of the SHARCS scaled rotor with three independent control systems," in $64^{\text {th }}$ Annual Forum of the Amerıcan Helıcopter Socıety, 2008.

[17] E. Ahci and R. Pfaller, "Structural design, optimization and validation of the integrated active trailing edge for a helicopter rotor blade," in $64^{\text {th }}$ Anual Forum of Amerıcan Helıcopter Socıety Internatıonal, (Montreal, Canada), 2008.

[18] J. Milgram and I. Chopra, "Helicopter vibration reduction with trailing edge flaps," $36^{\text {th }}$ AIAA/ASME/ASCE/AHS/ASC Structures, Structural Dynamics, and Maternals Conference, 1995. 
[19] F. Nitzsche and E. Breitbach, "Using adaptive structures to attenuate rotary wing aeroelastic response," Journal of Atrcraft, vol. 31. no. 5, pp. 1179-1188, 1994.

[20] J. Rodgers, Development of an integral twist-actuated rotor blade for individual control. PhD thesis, Massachusetts Institute of Technology, 1998.

[21] S. Shin, Integral twist actuation of helicopter rotor blades for vibratıon reduction. $\mathrm{PhD}$ thesis, Massachusetts Institute of Technology, 2001.

[22] J. Kim, Desıgn and analysis of rotor systems with multıple tralling edge flaps and resonant actuators. $\mathrm{PhD}$ thesis, The Pennsylvania State University, 2005.

[23] M. Spencer, R. Sanner, and I. Chopra, "Adaptive neurocontrol of simulated rotor vibrations using trailing edge flaps," Journal of Intelligent Matherial Systems and Structures, vol. 10, pp. 855-871, 1999.

[24] G. Depailler, Alleviatıon of dynamıc stall ınduced vibratıons in helıcopter rotors using actrvely controlled flaps. PhD thesis, The University of Michigan, 2002.

[25] C. Venkatesan and P. Friedmann, "New approach to finite-state modeling of unsteady aerodynamics," AIAA Journal, vol. 24, no. 12, pp. 1889-1897, 1996.

[26] D. Peters, M. Hsieh, and A. Torrero, "A state-space airloads theory for flexible airfoils," in $62^{\text {nd }}$ Annual Forum of American Helıcopter Socıety, (Phoenix, AZ.), 2006.

[27] M. Dinyavari and P. Friedmann, "Application of time-domain unsteady aerodynamics to rotary-wing aeroelasticity," AIAA Journal, vol. 24, no. 9, pp. 1424-1432, 1985.

[28] Timothy F. Myrtle and P. Friedmann, "Unsteady compressible aerodynamics of a flapped airfoil with application to helicopter vibration reduction," in AIAA/ASME/ASCE/AHS/ASC Structures, Structural Dynamıcs, and Materials Conference, pp. 224-240, 1997.

[29] P. Pawar, S. Jung, and Y. Yu, "Helicopter vibration reduction using active twist control of composite rotor blades," in $64^{\text {th }}$ Anual Forum of American Helicopter Society International, (Montreal, Canada), 2008. 
[30] N. Hariharan and J. Leishman, "Unsteady aerodynamics of a flapped airfoil in subsonic flow by indicial concepts," Journal of Aircraft, vol. 33, no. 5, pp. 855-868, 1996.

[31] S. R. Bhat and R. Ganguli, "Validation of comprehensive helicopter aeroelastic analysis with experimental data," Defence Science Journal, vol. 54, no. 4, pp. 419-427, 2004 .

[32] R. Ganguli, I. Chopra, and W. Weller, "Comparison of calculated vibratory rotor hub loads with experimental data," American Helicopter Society, vol. 43, no. 4, pp. 312$318,1998$.

[33] J. Milgram, I. Chopra, and F. Straub, "Rotors with trailing edge flaps: Analysis and comparison with experimental data," American Helicopter Society, vol. 43, no. 4, pp. 319-332, 1998.

[34] R. Steijl, G. Barakos, and K. Badcock, "A framework for CFD analysis of helicopter rotors in hover and forward flight," International Journal For Numerical Methods In Fluids, vol. 51, p. 819847, 2006.

[35] S. Voutsinas, "Vortex methods in aeronautics: How to make things work," International Journal of Computational Fluid Dynamics, vol. 20, no. 1, pp. 3-18, 2006.

[36] G. Oxley, A 2-D hybrid euler-compressible vortex particle method for transonic rotorcraft flows. $\mathrm{PhD}$ thesis, Carleton University, 2009.

[37] H. Ashley, E. Brunelle, and H. Moser, "Unsteady flow through helicopter rotors," Zeitschrift fr Angewandte Mathematik und Physik (ZAMP), vol. 9, no. 5-6, pp. 57$80,1958$.

[38] M. Ghorashi, Dynamics of elastic nonlinear rotating composite beams with embedded actuators. PhD thesis, Department of Mechanical and Aerospace EngineeringCarleton University, 2009.

[39] D. Hodges, "Review of composite rotor blade modeling," AIAA Journal, vol. 28, no. 3, pp. 561-565, 1990. 
[40] P. Friedmann and D. Hodges, "Rotary wing aeroelasticitya historical perspective," Journal of Aircraf, vol. 40, no. 6, pp. 1019-1046, 2003.

[41] S. Voutsinas and D.G.Triantos, "High resolution aerodynamic analysis of full helicopter configurations," in $25^{\text {th }}$ European Rotorcraft Forum, no. C-11, (Rome), 1999.

[42] S. Voutsinas, A. Visingardi, J. Yin, G. Arnaud, D. Falchero, A. Dummel, M. Pidd, and J. Prospathopoulos, "Aerodynamic interference in full helicopter configurations and assessment of noise emission: Pre-test modelling activities for the helinovi experimental campaign," in $31^{\text {st }}$ European Rotorcraft Forum, (Florence, Italy), 2005.

[43] O. Dieterich, H. Langer, O. Sneider, G. Imbert, M. Hounjet, V. Riziotis, I. Cafarelli, R. Calvo Alonso, C. Clerc, and K. Pengel, "Helinovi: Current vibration research activities," in $31^{\text {st }}$ European Rotorcraft forum, Florence, Italy, (Florence.Italy.), 2005.

[44] V. Riziotis and S. Voutsinas, "Advanced aeroelastic modelling of complete wind turbine configurations in view of assessing stability characteristics," in Proceedings of the EWEC, (Athens, Greece), 2006.

[45] J. Richards, Analysıs of Perıodıcally Tıme-Varyıng Systems. Springer-Verlag Berlin Heidelberg Newyork, 1983.

[46] H. D'Angelo, Lınear Tıme-Varyıng Systems: Analysıs and Synthesıs. Allyn and Bacon, 1970.

[47] R. Crochiere, Multırate Dıgıtal Sıgnal Processıng. Prentice Hall International, 1983.

[48] S. Bittanti and P. Colaneri, Perıodıc System: Fultering and Control. Springer London, 2009.

[49] B. Bamieh, J. B. Pearson, B. A. Francis, and A. Tannenbaum, "A lifting technique for linear periodic systems with applications to sampled-data control," Systems and Control Letters, vol. 17, pp. 79-88, 1991.

[50] T. Chen and B. Francis, Optımal Sampled-Data Control Systems. Communication and Control Engineering Series, Springer-Verlag, 1995. 
[51] P. Khargonekar, K. Poolla, and A. Tannenbaum, "Robust control of linear time invariant plants using periodic compansation," IEEE Transactions on Automatic Control, vol. 30, no. 11, pp. 1088-1096, 1985.

[52] C. Farges, Methodes d'analyse et de synthese robustes pour les systems lineaires periodiques. PhD thesis, l'Univesite Paul Sabatier de Touluse, 2006.

[53] E. Mollerstedt and B. Bernhardsson, "A harmonic transfer function model for a diode converter train," IEEE, Power Engineering Society Winter Meeting, vol. 2, pp. 957 $962,2000$.

[54] N. Wereley and S. Hall, "Linear control issues in the higher harmonic control of helicopter vibrations," in Proceedings of the $45^{\text {th }}$ Forum of the American Helicopter Society, (Boston, MA), May 1989.

[55] L. Zadeh, "Frequency analysis of variable networks," in Proc. Inst. Radio Engineering, 1950.

[56] N. Wereley, Analysis and control of linear periodically time varying systems. $\mathrm{PhD}$ thesis, Massachusetts Institute of Technology, February 1991.

[57] Y. Yamamato and M. Araki, "Frequency response of sampled data systems- their equivalence and relationships," Linear Algebra And Its Applications, vol. 205-206, pp. 1319-1339, 1994.

[58] J. Gibson, Nonlinear Automatic Control. McGraw-Hill, 1963.

[59] F. Nitzsche, "Laplace domain approximation to the transfer fuctions of a rotor blade in forward flight," The Aeronautical Journal, pp. 233-240, 2001.

[60] H. Sandberg, E. Mollerstedt, and B. Bernhardsson, "Frequency-domain analysis of linear time-periodic systems," Transactions on Automatic Control, vol. 50, no. 12, pp. 1971-1983, 2005.

[61] G. Adams and G. Goodwin, "Parameter estimation for periodic ARMA models," Journal Of Time Series Analysis, vol. 16, no. 2, pp. 127-145, 1995. 
[62] B. Bamieh and J. Pearson, "A general framework for linear periodic sampled-data control systems with applications to $H_{\infty}$ sampled-data systems," IEEE Transactrons On Automatıc Control, vol. 37, no. 4, pp. 418-435, 1992.

[63] R. Meyer and C. Burrus, "A unified analysis of multirate and periodically timevarying digital filters," IEEE Transactıons On Crrcurts And Systems, vol. 22, no. 3, pp. $162-168,1975$.

[64] L. Ljung, System Identrfication: Theory for the User. Prentice Hall, 1987.

[65] M. Gevers, "System identification without Lennart Ljung: What would have been different ?," in Workshop on system ıdentıficatıon un honor of Lennart Ljung, 2004.

[66] Ho and Kalman, "Effective construction of linear state-variable models from inputoutput functions," Regelungstechnık, vol. 12, pp. 545-548, 1965.

[67] A. Siddiqi, "Identification of the harmonic transfer function of a helicopter rotor," Master's thesis, Massachusetts Institute of Technology, 1999.

[68] S. Hwang and N. Wereley, "Frequency domain system identification of helicopter blades with trailing edge flaps," AIAA/ASME/AHS Adaptıve Structures Forum, 1996.

[69] M. Verhaegen and X. Yu, "A class of subspace model identification algorithms to identify periodically and arbitrarily time-varying systems," Automatıca, vol. 31, no. 2, pp. 201-216, 1995.

[70] H. Schwartz and K. Youcef-Toumi, "A recursive indentification controller for manipulater trajectory," in IEEE Conference on Decision and Control, 1987.

[71] C. Jimenez, A. McLeod, and K. Hipel, "Kalman filter estimation for periodic autoregressive-moving average models," in Stochastıc Hydrology and Hydraulics, vol. 3, (Salt Lake City, UT), pp. 227-240, Springer Verlag, 1989.

[72] J. Terceiro, "Comments on "Kalman-filtering methods for computing information matrices for time-invariant, periodic, and generally time-varying VARMA models and samples"," Computers and Mathematıcs with Applications, vol. 40, pp. 405-411, 2000 . 
[73] C. Lin and C. King, "Minimal periodic realizations of transfer matrices," IEEE Transactions on Automatic Control, vol. 38, no. 3, pp. 462-466, 1993.

[74] P. Colaneri and S. Longhi, "The realization problem for linear periodic systems," Automatica, vol. 31, no. 5, pp. 775-779, 1995.

[75] S. Wang and C. Zhang, "Minimal realization for lifted periodic filters," Circuits Systems Signal Process, vol. 19, no. 5, pp. 411-421, 2000.

[76] A. Varga, "Periodic Lyapunov equations: some applications and new algorihms," International Journal of Control, vol. 67, pp. 69-87, 1997.

[77] D. Flamm, "A new shift-invariant representation for periodic linear systems," Systems and Control Letters, vol. 17, pp. 9-14, 1991.

[78] H. Sandberg, "On floquet-fourier realizations of linear time-periodic impulse responses," in Proceedings of the $45^{\text {th }}$ IEEE Conference on Decision and Control, (San Diego, CA, USA.), December 2006.

[79] H. Zeiger and A. McEwen, "Approximate linear realizations of given dimension via Hos algorithm," Transactions on Automatic Control, vol. 19, p. 153, 1974.

[80] M. Moonen, B. De Moor, and J. Ramos, "A subspace identification algorithm for descriptor systems," Systems and Control Letters, vol. 19, pp. 47-52, 1992.

[81] T. Katayama, Subspace Methods for System Identification. Springer-Verlag London Limited, 2005.

[82] J. Hench and A. Laub, "Numerical solution of the discrete-time periodic Riccati equation," IEEE Transactions On Automatic Control, vol. 39, no. 6, pp. 1197-1210, 1994.

[83] P. Colaneri, R. Celi, and S. Bittanti, "Constant-coefficient representations of periodiccoefficient discrete linear systems," in $4^{\text {th }}$ Decennial Specialists Conference on Aeromechanics, AHS, (San Francisco, CA.), January 2004. 
[84] M. Lovera, Subspace Identıfication methods: theory and applications. $\mathrm{PhD}$ thesis, Politecnico Di Milano, 1997.

[85] V. Verdult, M. Lovera, and M. Verhaegen, "Identification of linear parameter-varying state-space models with application to helicopter rotor dynamics," Internatıonal Journal of Control. vol. 77, no. 13, p. 1149 1159, 2004.

[86] F. Felici, J. v. Wingerden, and M. Verhaegen, "Subspace identification of MIMO LPV systems using a periodic scheduling sequence," Automatıca, vol. 43, no. 10, pp. 1684$1697,2007$.

[87] J. v. Wingerden, I. Houtzager, F. Felici, and M. Verhaegen, "Closed-loop identification of the time-varying dynamics of variable-speed wind turbines," International Journal Of Robust And Nonlınear Control, vol. 19, pp. 4-21, 2009.

[88] G. Dullerud and F. Paganini, A Course in Robust Control Theory, A Convex Approach. Springer-Verlag, 1996.

[89] K. Zhou and J. Doyle, Essentıals of Robust Control. Prentice Hall International, 1997.

[90] K. Astrom and B. Wittenmark, Adaptıve Control. Addison-Wesley, 1989.

[91] B. Francis, $A$ Course in $H_{\infty}$ Control Theory. Springer-Verlag, 1987.

[92] D. Leith and W. Leithead, "Survey of gain-scheduling analysis and design," Internatronal Journal of Control, vol. 73, no. 11, pp. 1001-1025, 2000.

[93] G. Zames, "Feedback and optimal sensitivity: model reference transformations, multiplicative seminorms, and approximate inverses," IEEE Transactıons on Automatıc Control, vol. 26, pp. 301-320, 1981.

[94] G. Balas, R. Chiang, A. Packard, and M. Safanov, Robust Control Toolbox.

[95] M. Grimble and V. Kucera, Polynomıal Methods for Control Systems Design. Springer-Verlag, 1996.

[96] S. Boyd, L. El Ghaoui, E. Feron, and V. Balakrishnan, Linear Matrix Inequalıty ın Systems and Control Theory. SIAM, 1994. 
[97] L. E. Ghaoui and S. Niculescu, Advances in Linear Matrix Inequality Methods in Control. SIAM, 2000.

[98] A. Varga, "Computation of minimal periodic realizations of transfer-function matrices," IEEE Transactions on Automatic Control, vol. 49, no. 1, pp. 146-149, 2004.

[99] C. De Souza and Alexandre Trofino, "An LMI approach to stabilization of linear discrete-time periodic systems," International Journal of Control, vol. 73, no. 8, pp. 696-703, 2000.

[100] S. Lall and G. Dullerud, "An LMI solution to the robust synthesis problem for multirate sampled-data systems," Automatica, vol. 37, pp. 1909-1922, 2001.

[101] F. Ulker, V. Nalbantoglu, Y. Chen, D. Zimcik, and Y. Yaman, "Active vibration control of a smart fin," in $50^{\text {th }}$ AIAA/ASME/ASCE/AHS/ASC Structures, Structural Dynamics, and Materials Conference, 2009.

[102] Y. Chen, F. Ulker, V. Nalbantoglu, V. Wickramasinghe, D. Zimcik, and Y. Yaman, "Active control of smart fin model for aircraft buffeting load alleviation applications," Journal of Aircraft, vol. 46, no. 6, pp. 1965-1972, 2009.

[103] G. Dullerud, Control of Uncertain Sampled Data Systems. Birkhauser, 1996.

[104] C. Pirie, "Controller synthesis for uncertain time-varying discrete-time systems," Master's thesis, Applied Mathematics, University of Waterloo,, Waterloo, Ontario, Canada., 1999.

[105] G. Dullerud, Multi-rate and linear time varying synthesis tools manual v2.2, March 2004.

[106] S. Bittanti, A. Laub, and J. Williems, The Riccati Equation. Springer-Verlag, 1991.

[107] J. Hench and A. Laub, "Numerical solution of the discrete-time periodic Riccati equation," IEEE Transactions On Automatic Control, vol. 30, no. 6, pp. 1197-1210, 1994. 
[108] R. Granat, B. Kagstrom, and D. Kressner, "Matlab tools for solving periodic eigenvalue problems," in $3^{\text {rd }}$ IFAC Workshop PSYCO07, 2007.

[109] A. Varga, "A periodic systems toolbox for matlab," in Proccedings of IFAC'05 World Congress, (Prague, Czech Republik), 2005.

[110] A. Varga, "On solving periodic Riccati equations," Numerical Linear Algebra With Applications, vol. 15, p. 809835, 2008.

[111] P. Benner, R. Byers, R. Mayo, E. Quintana-Orti, and V. Hernandez, "Parallel algorithms for LQ optimal control of discrete-time periodic linear systems," Journal of Parallel and Distributed Computing, vol. 62, p. 306325, 2002.

[112] D. Patt, L. Liu, J. Chandrasekar, D. Bernstein, and P. Friedmann, "The HHC algorithm for helicopter vibration reduction revisited," in $45^{\text {th }}$ AIAA/ASME/ASCE/AHS/ASC Structures, Structural Dynamics and Materials Conference, 2004.

[113] L. Liu, P. Friedmann, I. Kim, and D. Bernstein, "Rotor performance enhancement and vibration reduction in presence of dynamic stall using actively controlled flaps," American Helicopter Society Journal, vol. 53, no. 4, pp. 338-350, 2008.

[114] B. Roget and I. Chopra, "Closed-loop test of a rotor with individually controlled trailing-edge flaps for vibration reduction," Journal Of The American Helicopter Society, vol. 55, pp. 1-12, 2009.

[115] T. Mannchen and K. Well, "Helicopter vibration reduction using periodic robust control," in AIAA Guidance, Navigation, and Control Conference and Exhibit, (Montreal, Canada), 2001.

[116] T. Mannchen and K. Well, "Helicopter vibration reduction and damping enhancement using individual blade control," Journal Of Guidance, Control, And Dynamics, vol. 27 , no. 5, pp. 760-766, 2004. 
[117] P. Arcara, S. Bittanti, and M. Lovera, "Periodic control of helicopter rotors for attenuation of vibrations in forward flight," IEEE Transactıons On Control Systems Technology, vol. 8, no. 6, pp. 883-894, 2000.

[118] S. Bittanti and F. Cuzzola, "Generalized active control of vibrations in helicopters," Journal of Guıdance, Control, and Dynamics, vol. 25, no. 2, pp. 340-351, 2002.

[119] G. Coppotelli, P. Marzocca, F. Ulker, J. Campbell. and F. Nitzsche, "Experimental investigation on modal signature of smart sprin and helicopter blade system," Journal of Arrcraft, vol. 45, no. 4, 2008.

[120] S. Bittanti and P. Colaneri, "Invariant representations of discrete-time periodic systems," Automatıca, vol. 36, pp. 1777-1793, 2000.

[121] P. Dewilde and A. Van Der Veen, Trme-Varyıng Systems and Computations. Kluwer Academic Publishers, 1998.

[122] S. Bittanti and F. Cuzzola, "An LMI approach to periodic discrete-time unbiased filtering," Systems and Control Letters, vol. 42, pp. 21-35, 2001.

[123] M. Verhaegen and A. Varga, "Some experience with the MOESP class of subspace model identfication methods in identifying the BO105 helicopter," tech. rep., DLR, German Aerospace Research Establishment, 1994.

[124] S. Boyd, L. E. Ghaoui, E. Feron, and V. Balakrishnan, Lınear Matrıx Inequalıtıes ın Systems and Control Theory. Society for Industrial and Applied Mathematics, SIAM, 1997.

[125] G. Dullerud and S. Lall, "A new approach for analysis and synthesis of time-varying systems," Ieee Transactıons On Automatıc Control, vol. 44, no. 8, pp. 1486-1497, 1999.

[126] A. Varga, "Balanced truncation model reduction of periodic systems," in Proceedings of the $39^{\text {th }}$ IEEE Conference on Decısion and Control, vol. 3, (Sydney, Australia), pp. $2379-2384,2000$. 
[127] T. Pappas and A. L. A. Sandell, "On the numerical solution of the discrete-time algebraic Riccati equation," IEEE Transactions on Automatic Control, vol. 25, pp. 631641, August 1980.

[128] A. Bojanczyk, G. Golub, and P. Van Dooren, "The periodic schur decomposition. Algorithms and applications," in SPIE Conference, vol. 1770, pp. 31-42, 1992.

[129] R. Granat, B. Kagstrom, and D. Kressner, "Reordering the eigenvalues of a periodic matrix pair with applications in control," in IEEE CACSD06, 2006.

[130] P. Crozier, P. Leconte, Y. Delrieux, B. Gimonet, A. Le Pape, and H. Mercier des Rochettes, "Wind-tunnel tests of a helicopter rotor with active flaps," in $32^{\text {nd }}$ European Rotorcraft Forum, (Maastricht, The Netherlands.), September 2006.

[131] A. Stoorvogel, "The singular $H_{2}$ control problem," Automatica, vol. 28, no. 3, pp. 627631, 1992.

[132] B. Chen and A. Saberi, "Necessary and sufficient conditions under which an $H_{2}$ optimal control problem has a unique solution," International Journal of Control, vol. 58, pp. 337-348, 1993.

[133] A. Farag and H. Werner, " $K-S-\phi$ iteration for robust $H_{2}$ controller synthesis," in European Control Conference ECC, (University of Cambridge, UK), 2003.

[134] D. Banjerdpongchai and J. How, "Parametric robust $H_{2}$ control design with generalized multipliers via LMI synthesis," International Journal of Control, vol. 70, no. 3, pp. 481-503, 1998.

[135] P. Gahinet, A. Nemirovski, A. Laub, and M. Chilali, LMI Control Toolbox.

[136] A. Farag and H. Werner, "A riccati-genetic algorithms approach to fixed-structure controller synthesis," in American Control Conference, (Boston, Massaschusetts), June 2004.

[137] M. Moonen, B. De Moor, L. VandenBerghe, and J. Vandewalle, "On- and off-line identification of linear state-space models," International Journal for Control, vol. 49, no. 1, pp. 219-232, 1989. 
[138] M. Tischler and R. Remple, Aırcraft and Rotorcraft System Identificatıon Engıneering Methods with Flight Test Examples. American Institute of Aeronautics and Astronautics, 2006 .

[139] P. Hamel and J. Kaletka, "Advances in rotorcraft system identification," Progress ın Aerospace Scıence, vol. 33, pp. 259-284, 1997.

[140] P. Hamel and R. Jategaonkar, "The role of system identification for flight vehicle applications - revisited," in RTO SCI Symposıum, System Identıficatıon for Integrated Atrcraft Development and Flight Testıng, (Madrid, Spain), 1998.

[141] G. E. Dullerud, "A new approach for analysis and synthesis of time-varying systems," IEEE, Transactıons of Automatıc Control, vol. 44, no. 4, pp. 1486-1497, 1999. 